Florida International University

FIU Digital Commons

$11-12-2019$

\title{
Consequences of Non-physiological Ligands Binding to DREAM on Its Secondary and Tertiary Structures and Interactions with Intracellular Partners.
}

Samiol Azam

sazam002@fiu.edu

Follow this and additional works at: https://digitalcommons.fiu.edu/etd

Part of the Chemistry Commons

\section{Recommended Citation}

Azam, Samiol, "Consequences of Non-physiological Ligands Binding to DREAM on Its Secondary and Tertiary Structures and Interactions with Intracellular Partners." (2019). FIU Electronic Theses and Dissertations. 4325.

https://digitalcommons.fiu.edu/etd/4325

This work is brought to you for free and open access by the University Graduate School at FIU Digital Commons. It has been accepted for inclusion in FIU Electronic Theses and Dissertations by an authorized administrator of FIU Digital Commons. For more information, please contact dcc@fiu.edu. 


\section{FLORIDA INTERNATIONAL UNIVERSITY}

Miami, Florida

CONSEQUENCES OF NON-PHYSIOLOGICAL LIGANDS BINDING TO DREAM ON ITS SECONDARY AND TERTIARY STRUCTURES AND INTERACTIONS

WITH INTRACELLULAR PARTNERS

A dissertation submitted in partial fulfillment of the requirements for the degree of

DOCTOR OF PHILOSOPHY

in

CHEMISTRY

by

Samiol Azam 
To: Dean Michael R. Heithaus

College of Arts, Sciences and Education

This dissertation, written by Samiol Azam, and entitled Consequences of Nonphysiological Ligands Binding to DREAM on Its Secondary and Tertiary Structures and Interactions with Intracellular Partners., having been approved in respect to style and intellectual content, is referred to you for judgment.

We have read this dissertation and recommend that it be approved.

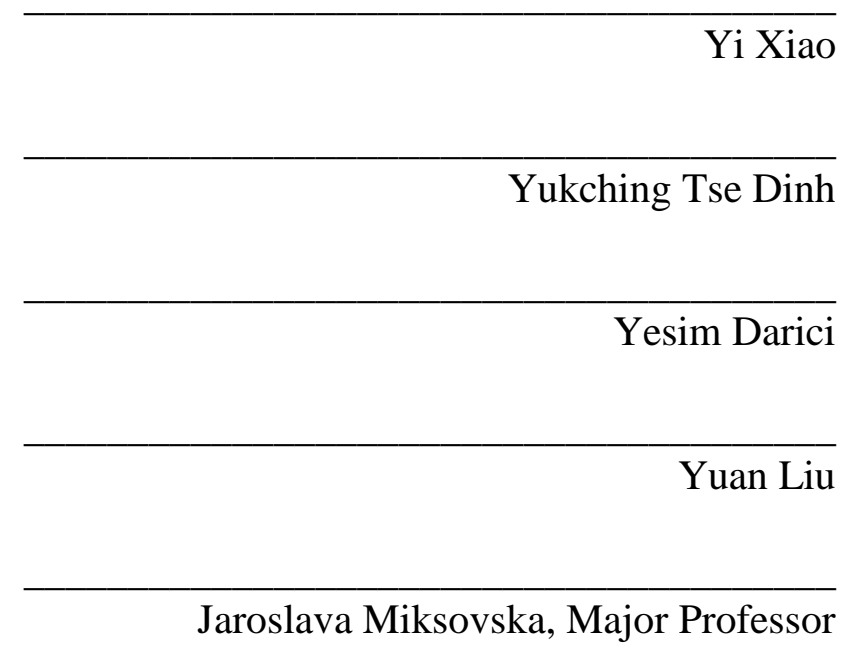

Date of Defense: November 12, 2019

The dissertation of Samiol Azam is approved.

Dean Michael R. Heithaus College of Arts, Sciences and Education

Andrés G. Gil

Vice President for Research and Economic Development and Dean of the University Graduate School

Florida International University, 2019 
(C) Copyright 2019 by Samiol Azam

All rights reserved. 


\section{DEDICATION}

This dissertation is dedicated to my elder brother, Mohammad Shamim Al Mamun, who passed away in 2010 in a tragic road accident. Also dedicated to my parents, Dr. Shah Alam and Ms. Alia Begum, whose main goal in life was to provide me the best possible education. 


\section{ACKNOWLEDGMENTS}

At first, I would like to thank my principal investigator, Dr. Jaroslava Miksovska, for giving me the opportunity to work on an exciting project. I appreciate his tremendous support for training me in the lab, designing the projects, and helping me publishing articles. Special thanks to former graduate student of our group Dr. Walter Gonzalez who was kind enough for providing me all lab training. I express my gratitude to all committee members, Dr. Yesim Darici, Dr. Yukching Tse Dinh, Dr. Yuan Liu, and Dr. Yi Xiao, for their time and constructive suggestions during original and research proposals, candidacy exam, and annual evaluations. I thank all former (Dr. Walter Gonzalez, Dr. David Butcher, Dr. Anthonija Tangar, Dr. Khoa Pham) and current graduate students (Ruipeng Lei, Maria Santiago) of Dr. Miksovska research laboratory for their helpful suggestions and making the laboratory a fun place to work for. Thanks to undergraduate students Devin Seka, Gessica St. Louis, Adriana Riveron, and Sasha Rodriguez who generously helped me in the cell culture experiments. Finally, I acknowledge FIU graduate school, College of Arts Science and Education (CASE), and Department of Chemistry for providing me teaching assistantship. 


\title{
ABSTRACT OF THE DISSERTATION
}

\section{CONSEQUENCES OF NON-PHYSIOLOGICAL LIGANDS BINDING TO DREAM ON ITS SECONDARY AND TERTIARY STRUCTURES AND INTERACTIONS WITH INTRACELLULAR PARTNERS.}

by

\author{
Samiol Azam \\ Florida International University, 2019 \\ Miami, Florida \\ Professor Jaroslava Miksovska, Major Professor
}

Downstream regulatory antagonist modulator (DREAM) is $29 \mathrm{kDa}$ neuronal calcium sensor (NCS) protein which is expressed in the hippocampus of the brain. The DREAM is involved in a wide spectrum of biological processes, including regulation of potassium current in brain, modulation of pain, presenilin-1 processing, regulation of memory and learning. Exposure to toxic metals such as lead $\left(\mathrm{Pb}^{2+}\right)$ and cadmium $\left(\mathrm{Cd}^{2+}\right)$ have been associated with different types of neurodegenerative diseases. However, how these toxic metals induce neurotoxicity remains an active area of research. In the present study, utilizing a combination of spectroscopic and calorimetric techniques, we demonstrated binding of these toxic metals to DREAM. We showed that $\mathrm{Pb}^{2+}$ and $\mathrm{Cd}^{2+}$ bind to EF-hands in DREAM and binding of these toxic metals alters DREAM secondary and tertiary structure. Lead and cadmium association to DREAM also modulates DREAM interactions with FITC-labeled peptides that mimic binding sites of DREAM effector proteins, presenilin 1 and $\mathrm{Kv}$ channel in a similar way as $\mathrm{Ca}^{2+}$ binding. Considering the high 
sequence homology between DREAM and other NCS proteins, $\mathrm{Pb}^{2+}$ and $\mathrm{Cd}^{2+}$ could bind to other NCS proteins and interactions of $\mathrm{Pb}^{2+}$ and $\mathrm{Cd}^{2+}$ to with $\mathrm{NCS}$ proteins could provide novel insight into the molecular mechanism of $\mathrm{Pb}^{2+}$ and $\mathrm{Cd}^{2+}$-induced neurotoxicity.

Lithium $\left(\mathrm{Li}^{+}\right)$has been used for the treatment of mental disorders for more than six decades. Increasing evidences from in vivo and in vitro studies have implicated that $\mathrm{Li}^{+}$is a drug candidate for the treatment of neurogenerative diseases. However, the molecular mechanism through which $\mathrm{Li}^{+}$exerts its therapeutic action has not elucidated yet. Here we investigated whether $\mathrm{Li}^{+}$directly binds to DREAM and impact its structural and functional properties. We demonstrated that $\mathrm{Li}^{+}$binding triggers structural rearrangement of DREAM and enhances DREAM interactions with site- 1 and site- 2 of the voltage-gated potassium channel. Results from this study suggest that DREAM and probably other NCS proteins are molecular targets of $\mathrm{Li}^{+}$and binding of $\mathrm{Li}^{+}$to DREAM could provide a novel insight into the molecular mechanism of $\mathrm{Li}^{+}$therapeutic action. 


\section{TABLE OF CONTENTS}

CHAPTER

PAGE

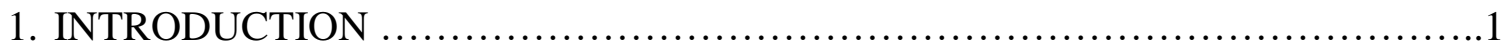

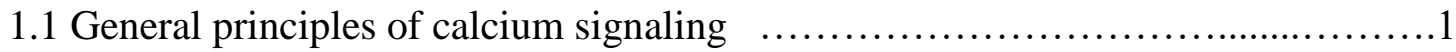

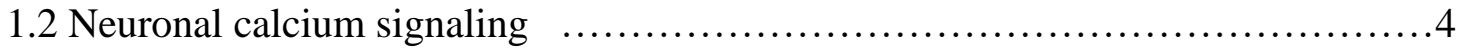

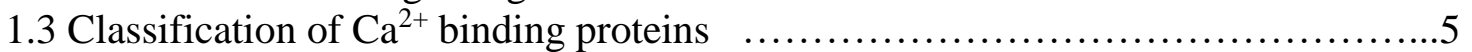

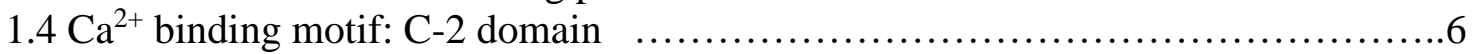

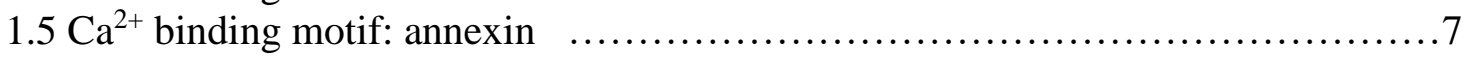

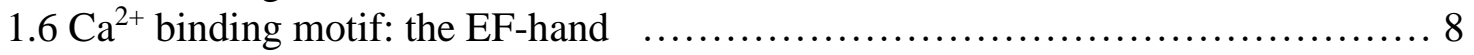

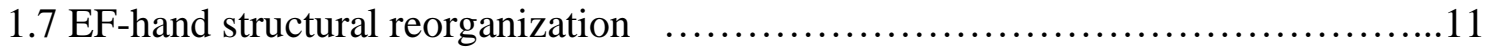

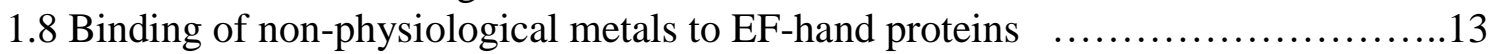

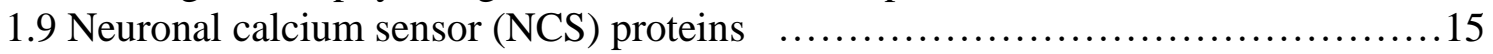

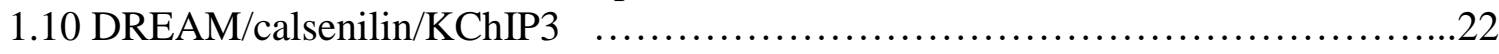

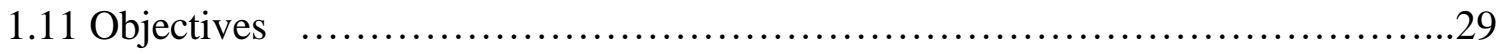

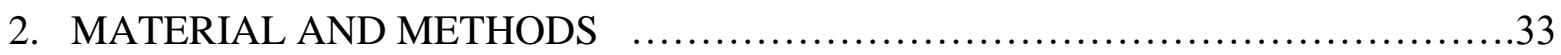

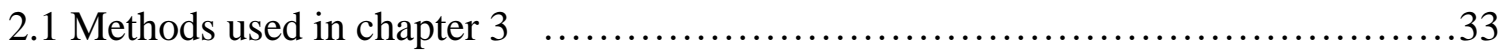

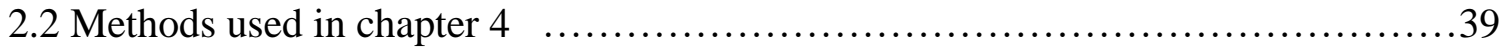

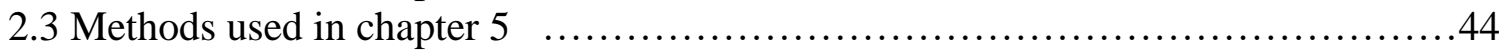

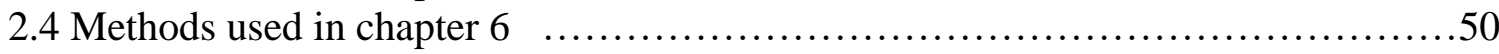

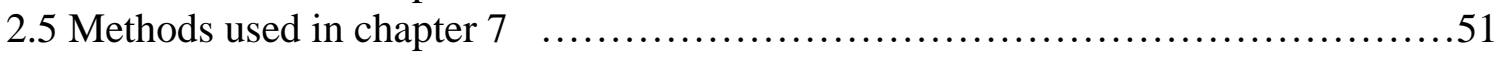

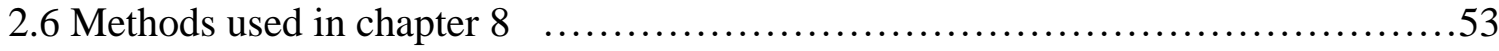

3. LEAD BINDS TO DREAM AND MODULATES ITS INTERACTIONS WITH BINDING PARTNERS: A LINK BETWEEN NEURONAL CALCIUM SENSORS

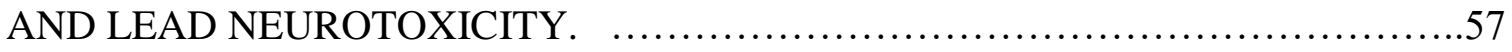

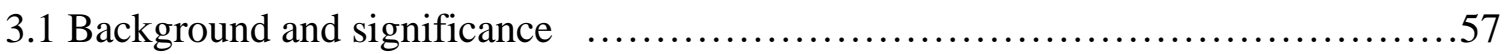

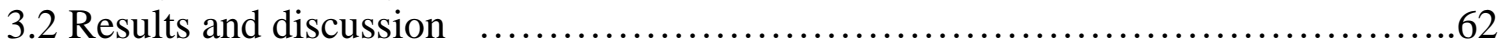

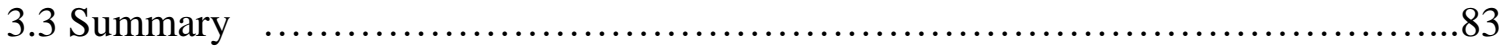

4. CADMIUM ASSOCIATION TO DREAM PROMOTES DREAM INTERACTIONS WITH INTRACELLULAR PARTNERS IN A SIMILAR MANNER AS ITS

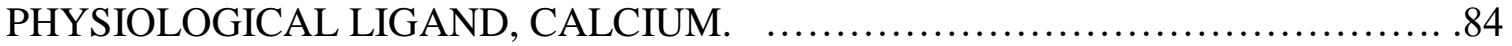

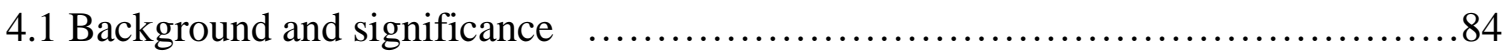

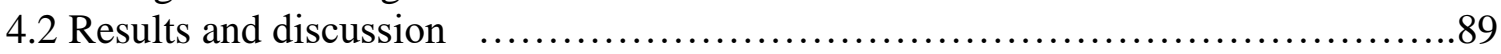

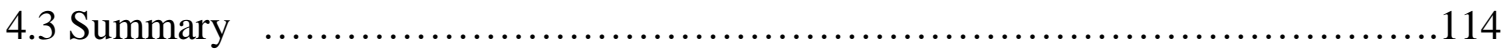

5. BINDING OF THE NEUROPROTECTIVE AGENT LITHIUM TO NEURONAL CALCIUM SENSOR PROTEIN DREAM: A NEW INSIGHT INTO LITHIUM THERAPY.

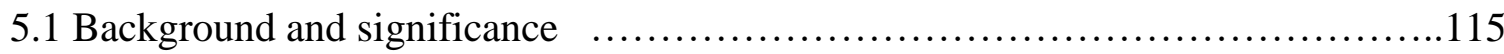

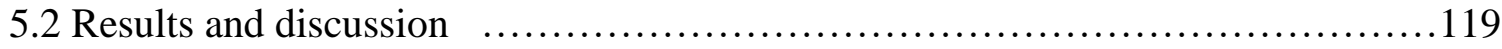

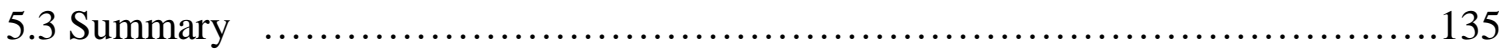


6. INVESTIGATION OF FEW OTHER METALS BINDING TO DREAM. . ..........136

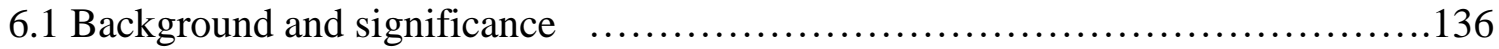

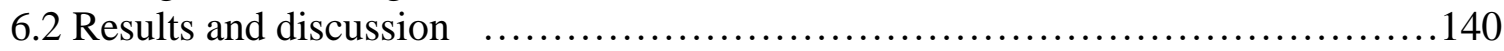

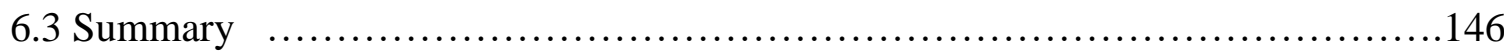

7. ROLE OF LYS RESIDUE AT POSITION 87 OF DREAM IN ALLOSTERIC REGULATION OF DREAM INTERACTIONS WITH INTERACTING PARTNERS. . 147

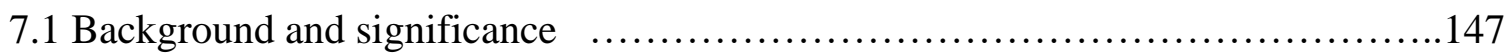

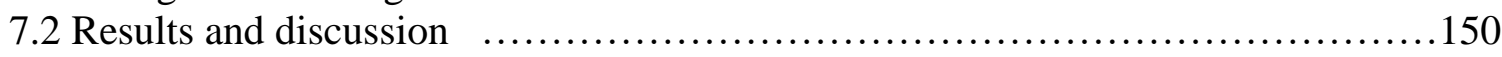

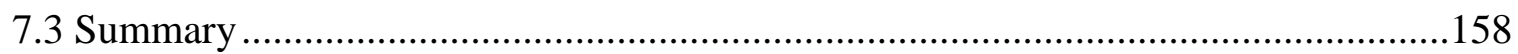

8. IMPACT OF EF-3 OR EF-4 INACTIVATION OF DREAM ON ITS SECONDARY AND TERTIARY STRUCTURES AND INTERACTIONS WITH T-1 DOMAIN OF

KV CHANNEL ................................................................. 159

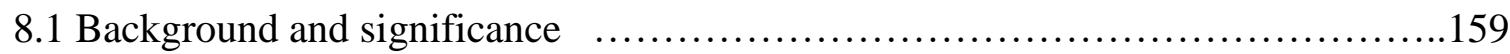

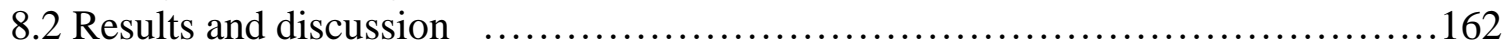

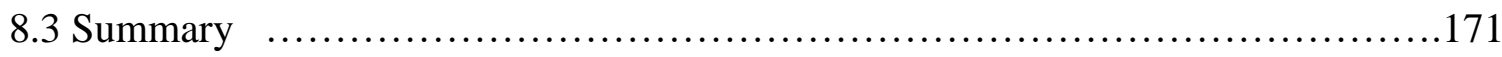

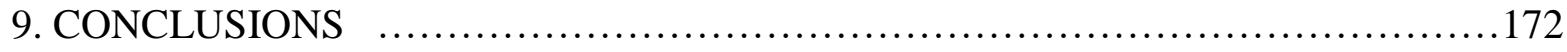

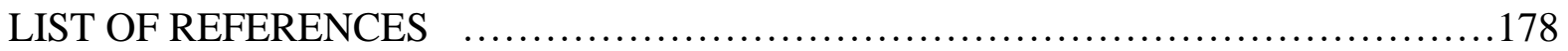

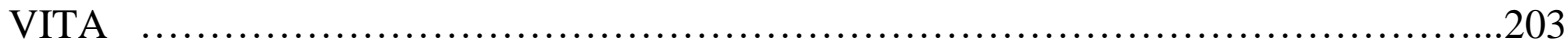




\section{LIST OF TABLES}

TABLE

PAGE

Table 3.1. Fluorescence decay parameters for $\operatorname{DREAM}(\Delta 64)$ as a function of $\mathrm{Ca}^{2+/} \mathrm{Pb}^{2+}$ binding to EF-hands.

Table 3.2. Fluorescence decay parameters for DREAM $(\Delta 64)-1,8-A N S$ complexes as a function of $\mathrm{Ca}^{2+}$ and/or $\mathrm{Pb}^{2+}$ binding to EF-hands.

Table 3.3. ITC parameters recovered for $\mathrm{Tb}^{3+}$ and $\mathrm{Pb}^{2+}$ displacement of $\mathrm{Ca}^{2+}$ from EF hands of DREAM using a sequential model.

Table 3.4. Equilibrium dissociation constants for presenilin-1 helix 9 "PS1HL9", Kv4.3(2-22) "site-1", and Kv4.3(70-90) "site-2" binding to DREAM constructs.

Table 4.1. Fluorescence decay parameters of WTDREAM $(\Delta 64)$ in the presence and absence of $\mathrm{Ca}^{2+}$ and $\mathrm{Cd}^{2+}$.

Table 4.2. Fluorescence decay parameters of WTDREAM $(\Delta 64): 1,8-\mathrm{ANS}$ complexes.

Table 4.3. Thermodynamic parameters determined for displacement of $\mathrm{Ca}^{2+}$ by $\mathrm{Cd}^{2+}$ from EF hands of DREAM.

Table 4.4. The equilibrium dissociation constants for PS1HL9, site-1 site-2 binding to DREAM constructs.

Table 5.1. Thermodynamics parameters recovered for $\mathrm{Li}^{+}$binding to EF-hand of metal-free DREAM.

Table 5.2. Fluorescence decay parameters for $\mathrm{Li}^{+}$and/or $\mathrm{Ca}^{2+}$ binding to $\operatorname{DREAM}(\Delta 64)-1,8$-ANS complexes. 
Table 7.1: Fluorescence decay parameters of DREAM(K87A) in the presence and absence of metals. ..............................................

Table 7.2: Fluorescence decay parameters of DREAM(K87A):1,8-ANS complexes.

Table 8.1: Fluorescence decay parameters of DREAM constructs in the presence and absence of metals.

Table 8.2: Fluorescence decay parameters of DREAM:1,8-ANS complexes. .... 169 


\section{LIST OF FIGURES}

FIGURE

PAGE

Figure 1.1. Components of $\mathrm{Ca}^{2+}$ signaling network. Maintenance of intracellular $\mathrm{Ca}^{2+}$ concentration is determined by the balance between ON/OFF reaction. Adapted from (Berridge, Lipp et al. 2000).

Figure 1.2. Three-dimensional structure of synaptotagmin-1 with $\mathrm{Ca}^{2+}$ bound to $\mathrm{C} 2 \mathrm{~B}$ domain (PDB: 1tjx). Cartoon representation of protein is shown as purple blue; $\mathrm{Ca}^{2+}$ is shown as red spheres.

Figure 1.3. Three-dimensional structure of $\mathrm{Ca}^{2+}$ bound annexin V (PDB: 1yii). Protein is represented with purple blue cartoon; $\mathrm{Ca}^{2+}$ is represented with red spheres.

Figure 1.4. Single canonical EF-hand of parvalbumin (PDB: 1b8r). Entering and exiting helices are shown as cyan; $\mathrm{Ca}^{2+}$ chelating loop is shown as magenta; $\mathrm{Ca}^{2+}$ ion is shown as green sphere, $\beta$-sheet is shown as red. . . 9

Figure 1.5. Schematic diagram of the coordination sphere of canonical EF-hand loop. Blue represents coordinating ligands, coordinating water molecule is shown in dark blue. Light green represents conserved glycine residue which allows bending of the loop. Entering and exiting helices are shown in red. Figure adapted from (Gifford, Walsh et al. 2007).

Figure 1.6. Structural reorganization of the EF-hands of CaM. Left) EF-1 in apo and $\mathrm{Ca}^{2+}$ bound forms are shown as firebrick color and slate colors, respectively. Right) EF-3 in apo and $\mathrm{Ca}^{2+}$-bound forms are shown as lime green and cyan, respectively. In both cases, $\mathrm{Ca}^{2+}$ is shown as magenta sphere. $\mathrm{Ca}^{2+}$ binding reorganizes both $\mathrm{EF}$-hands from antiparallel to perpendicular orientation. PDB code of apo-CaM: $1 \mathrm{cfc}$; PDB code of $\mathrm{Ca}^{2+}-\mathrm{CaM}$ : 1 cll.

Figure 1.7. Structural reorganization of the EF-hands of recoverin. A) EF-1 in apo and $\mathrm{Ca}^{2+}$ bound forms are shown as firebrick and slate, respectively. B) EF-3 in apo and $\mathrm{Ca}^{2+}$-bound forms are shown as lime green and cyan, respectively. In both cases, $\mathrm{Ca}^{2+}$ is shown as magenta sphere. $\mathrm{Ca}^{2+}$ binding does not lead to significant structural reorganization in the 
protein. PDB code of apo-recoverin: 1iku; PDB code of $\mathrm{Ca}^{2+}$-recoverin: 1 jsa.

Figure 1.8. NMR structure of NCS-1 protein (PDB: 2lcp). EF-hands 1, 2, 3, and 4 are shown as firebrick, slate, lime green, and cyan, respectively; $\mathrm{Ca}^{2+}$ ions are shown as magenta spheres; and rest of the protein is shown as orange. EF-1 and EF-2 constitutes N terminus; EF-3 and EF-4 constitutes $\mathrm{C}$-terminus. In each terminus, paired EF-hands communicate through a short antiparallel $\beta$-sheet.

Figure 1.9. Left: NMR structure of $\mathrm{Ca}^{2+}$-free recoverin (PDB code: 1iku). Right: NMR structure of $\mathrm{Ca}^{2+}$-bound recoverin (PDB code: $1 \mathrm{jsa}$ ). In both cases, EF-hands 1, 2, 3, and 4 are shown as firebrick, slate, lime green, and cyan, respectively. Rest of the protein is shown as cyan. $\mathrm{Ca}^{2+}$ ions are shown as magenta sphere. Myristoyl group is shown as pink. Myristoyl group is buried in $\mathrm{Ca}^{2+}$-free recoverin, but $\mathrm{Ca}^{2+}$ binding exposes the myristoyl group.

Figure 1.10. Representation of the amino acid residues of KChIP1 involved in interaction with $\mathrm{N}$-terminus of Kv4.3 channel (PDB: 2nz0). $\mathrm{N}$ terminus of the protein is shown in firebrick, $\mathrm{C}$-terminus is shown in slate. Residues involved in first interface is shown in cyan sticks, residues involved in the second interface is shown in lime green sticks.

Figure1.11. Cartoon representation of $\mathrm{Ca}^{2+}$-bound DREAM(78-256) (PDB: 2jul). EF-hands 1, 2, 3, and 4 are shown in orange, red, green, and magenta, respectively. $\mathrm{Ca}^{2+}$ ions are shown in cyan spheres. Rest of the protein is shown in blue. For clariy, structure of first 77 residues were not resolved.

Figure 1.12. The central hydrophobic patch of the DREAM that communicate between $\mathrm{N}$ - and $\mathrm{C}$ - teriminal domain is shown in stick (PDB: 2jul). EFhands 1, 2, 3, and 4 are shown in diffuse firebrick, slate, lime green, and cyan, respectively. Rest of the protein is shown in orange. $\mathrm{Ca}^{2+}$ ions are shown in magenta spheres.

Figure 1.13. The C-terminal hydrophobic patch of DREAM is shown in stick (PDB: 2jul). EF-hands 1, 2, 3, and 4 are shown in diffuse firebrick, slate, 
lime green, and cyan, respectively. Rest of the protein is shown in orange. $\mathrm{Ca}^{2+}$ ions are shown in magenta spheres.

Figure 1.14. Model structure of DREAM dimer based on the NMR structure of DREM monomer (PDB entry 2JUL). Left panel shows hydrophobic interactions between Leu 155, 159 and 251 and right panel show a salt bridge between Arg 200, Arg 207 (in blue) and Glu 103 (in red). The two dimers were docked using Autodock and the initial structure of the dimer was refined in $50 \mathrm{~ns}$ molecular dynamic simulation.

Figure 1.15. Cartoon representation of the N-terminus of DREAM (PDB: 2jul). Cluster of basic amino acid residues that are proposed to interact with DNA is shown as stick.

Figure 3.1 Structure of $\mathrm{Ca}^{2+}$ bound DREAM (PDB entry 2JUL). The four EFhands of DREAM are colored in orange (EF-hand 1), green (EF-hand 2), blue (EF-hand 3), and red (EF-hand 4). The helix-1 and helix-10 are shown in cyan. $\mathrm{Ca}^{2+}$ ions bound to EF-hand 3 and EF-hand 4 are shown as yellow spheres.

Figure 3.2. Fluorescence emission spectra of $20 \mu \mathrm{M}$ DREAM $(\Delta 64)$ in the metal free form and in the presence of $\mathrm{Ca}^{2+}$ and/or $\mathrm{Pb}^{2+}$. Conditions: $20 \mathrm{mM}$ Tris buffer (pH 7.4), 1mM EDTA, $1 \mathrm{mM} \mathrm{Ca}^{2+}, 40 \mu \mathrm{M} \mathrm{Pb}^{2+}$, and $1 \mathrm{mM}$ $\mathrm{Ca}^{2+}$ and $40 \mu \mathrm{M} \mathrm{Pb}^{2+}$; samples were excited at $295 \mathrm{~nm}$ and experiments were carried out at $20^{\circ} \mathrm{C}(\mathrm{A})$. Titration curve for $\mathrm{Pb}^{2+}$ binding to $\mathrm{Ca}^{2+}$ DREAM. The fraction bound was determined based on decrease in Trp169 emission intensity at increasing $\mathrm{Pb}^{2+}$ concentration. Conditions: $20 \mu \mathrm{M}$ DREAM $(\Delta 64)$ in $5 \mathrm{mM}$ Tris, $\mathrm{pH} 7.4$ and $100 \mu \mathrm{M} \mathrm{Ca}^{2+}$.

Experimental points correspond to the averadge value determined from three independent measurments. The solid line represents the fit using Equation 2; the standard deviations are shown as error bars (B). Fluorescence emission spectra of $20 \mu \mathrm{M} \operatorname{DREAM}(\Delta 160)$. Conditions as described for Figure 3.2A (C).

Figure 3.3. (A) Far-UV CD spectra of $20 \mu \mathrm{M}$ DREAM( $\triangle 64)$ in the presence of $1 \mathrm{mM}$ EDTA, $1 \mathrm{mM} \mathrm{Ca}^{2+}, 40 \mu \mathrm{M} \mathrm{Pb}^{2+}$, and $\left(1 \mathrm{mM} \mathrm{Ca}^{2+}\right.$ and $\left.40 \mu \mathrm{M} \mathrm{Pb}^{2+}\right)$ in $20 \mathrm{mM}$ Tris (pH 7.4). (B) Near-UV CD spectra of $40 \mu \mathrm{M}$ $\operatorname{DREAM}(\Delta 64)$ in the presence of $1 \mathrm{mM}$ EDTA, $1 \mathrm{mM} \mathrm{Ca}^{2+}, 40 \mu \mathrm{M} \mathrm{Pb}^{2+}$, and $\left(1 \mathrm{mM} \mathrm{Ca}^{2+}\right.$ and $\left.40 \mu \mathrm{M} \mathrm{Pb}^{2+}\right)$ in $5 \mathrm{mM}$ phosphate ( $\left.\mathrm{pH} 7.4\right)$. 
Figure 3.4. Frequency-domain intensity decay of $20 \mu \mathrm{M}$ apo (squares), $\mathrm{Ca}^{2+}$ (circle), $\mathrm{Pb}^{2+}$ (up triangle), and $\mathrm{Ca}^{2+} \mathrm{Pb}^{2+}$ (down triangle)DREAM $(\Delta 64)$ in $20 \mathrm{mM}$ Tris ( $\mathrm{pH} 7.4)$. The phase delay is shown as solid symbols and modulation ratio as empty symbols. Solid lines represent data fitting using a (one Gaussian distribution and single exponential decay) model.

Figure 3.5. Emission spectra of (A) 1,8-ANS:DREAM( $\Delta 64)$ and (B) 1,8ANS:DREAM $(\Delta 160)$ complexes as a function of $\mathrm{Pb}^{2+}$ or $\mathrm{Ca}^{2+}$ binding to the protein. Conditions: $20 \mu \mathrm{M}$ DREAM $(\Delta 64)$ and $20 \mu \mathrm{M} 1,8-\mathrm{ANS}$; excited at $350 \mathrm{~nm}$. (C) Titration of $10 \mu \mathrm{M}$ 1,8-ANS with $\mathrm{Pb}^{2+} \mathrm{DREAM}(\Delta 64)$. The fit of the experimental data using Equation 2 is shown as a solid line. Inset: 1,8 -ANS structure.

Figure 3.6. Frequency-domain intensity decay of $40 \mu \mathrm{M} 1,8$-ANS binding to 40 $\mu \mathrm{M}$ DREAM $(\Delta 64)$ in presence of EDTA (squares), $\mathrm{Ca}^{2+}$ (circle), $\mathrm{Pb}^{2+}$ (up triangle), and $\mathrm{Ca}^{2+} \mathrm{Pb}^{2+}$ (down triangle). The phase delay is shown as solid symbols and modulation ratio as empty symbols. Solid lines represent data fitting using three-exponential decay model.

Figure 3.7. ITC isotherms for $\mathrm{Pb}^{2+}$ displacement of $\mathrm{Ca}^{2+}$ from (A) DREAM $(\Delta 64)$ and $(B)$ DREAM $(\Delta 160)$. The upper panels of the profile reflect the thermal power expressed in units of microcalories per second, whereas the lower panel represents integrated reaction heats $(\Delta \mathrm{H})$ expressed in units of kilocalories per mole. The solid line represents the best fitting curve with parameters listed in Table 3.3.

Figure 3.8A,B. Titration of $0.5 \mu \mathrm{M}$ PS1HL9 with $\mathrm{Pb}^{2+}$ DREAM $(\Delta 64)$. Left panel represents raw anisotropy data. Right panel shows fraction bound that was calculated using Equation 1. In both cases, solid lines correspond to fit of the experimental data using Equation 2.

Figure 3.9A,B. Titration of $0.5 \mu \mathrm{M} \mathrm{Kv} 4.3$ (2-22) "site-1" with $\mathrm{Pb}^{2+} \operatorname{DREAM}(\Delta 64)$. Right panel shows fraction bound that was calculated using Equation 1. In both cases, solid lines correspond to fit of the experimental data using Equation 2.

Figure 3.10A,B. Titration of $0.5 \mu \mathrm{M} \mathrm{Kv} 4.3$ (70-90) "site-2" with $\mathrm{Pb}^{2+}$ DREAM $(\Delta 64)$. Right panel shows fraction bound that was 
calculated using Equation 1. In both cases, solid lines correspond to fit of the experimental data using Equation 2.

Figure 4.1. NMR structure of $\mathrm{Ca}^{2+}$-bound DREAM monomer (PDB entry 2JUL). The four EF-hands of DREAM are shown in raspberry (EF-hand 1), slate (EF-hand 2), teal (EF-hand 3), and firebrick (EF-hand 4); $\mathrm{Ca}^{2+}$ ions are shown as magenta spheres; W169 is shown as limon stick; and rest of the protein is shown in limon.

Figure 4.2. Effects of $\mathrm{Ca}^{2+}$ and $\mathrm{Cd}^{2+}$ on emission properties of $\mathrm{W} 169$ in (A) $\operatorname{DREAM}(\Delta 64),(\mathrm{B}) \operatorname{DREAM}(\Delta 160),(\mathrm{C}) \mathrm{DREAM}(\mathrm{E} 186)$, and (D) DREAM(E234Q). Conditions: $20 \mu \mathrm{M}$ protein in $20 \mathrm{mM}$ Tris $\mathrm{pH} 7.4,2$ mM EDTA or $2 \mathrm{mM} \mathrm{Ca}^{2+}$, and/or $100 \mu \mathrm{M} \mathrm{Cd}^{2+}$. Emission spectra were recorded using $295 \mathrm{~nm}$ excitation light.

Figure 4.3. Emission spectra of $\operatorname{DREAM}(\Delta 64)$ as a function of increasing concentration of $\mathrm{Cd}^{2+}$. Conditions: $20 \mu \mathrm{M}$ WTDREAM( $\left.\Delta 64\right)$ in $20 \mathrm{mM}$ Tris in presence of $100 \mu \mathrm{M} \mathrm{Ca}^{2+}$. The emission spectra were recorded using $295 \mathrm{~nm}$ excitation (A). The decrease in the emission intensity of $\operatorname{DREAM}(\Delta 64)$ due to the $\mathrm{Cd}^{2+}$ binding to $\mathrm{Ca}^{2+}$ DREAM. The experimental data were fitted using Equation 1 for obtaining equilibrium dissociation constant (B).

Figure 4.4. Far-UV CD spectra of (A) $20 \mu \mathrm{M}$ DREAM( $\Delta 64)$, (B) $20 \mu \mathrm{M}$ $\operatorname{DREAM}(\Delta 160),(\mathrm{C}) 20 \mu \mathrm{M}$ DREAM(E186), and (D) $20 \mu \mathrm{M}$ DREAM(E234Q). Conditions as described in Figure 4.2.

Figure 4.5. Frequency-domain intensity decay of $20 \mu \mathrm{M}$ apoDREAM $(\Delta 64)$ (square), $\mathrm{Ca}^{2+} \operatorname{DREAM}(\Delta 64)$ (circle), $\mathrm{Cd}^{2+} \mathrm{DREAM}(\Delta 64)$ (up triangle), and $\mathrm{Cd}^{2+} \mathrm{Ca}^{2+}$ DREAM $(\Delta 64)$ (down triangle). Solid symbols represent phase delay, whereas empty symbols represent modulation ratio. Solid lines correspond to the fitting of the data using a sum of three exponential decay model.

Figure 4.6. Emission spectra of (A) 1,8-ANS:DREAM( $\Delta 64)$, (B) 1,8ANS:DREAM( $\triangle 160)$, (C) 1,8-ANS:DREAM(E186), and (D) 1,8ANS:DREAM(E234Q) complexes. Conditions: Conditions: $20 \mu \mathrm{M}$ protein in $20 \mathrm{mM}$ Tris $\mathrm{pH} 7.4,20 \mu \mathrm{M}$ 1,8-ANS, $2 \mathrm{mM}$ EDTA or $2 \mathrm{mM}$ 


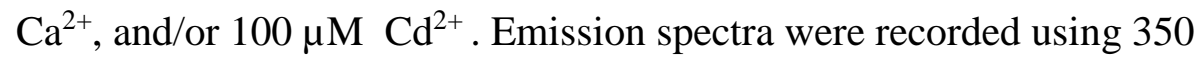
nm excitation light.

Figure 4.7. Titration curve for 1,8 -ANS binding to $\mathrm{Cd}^{2+} \operatorname{DREAM}(\Delta 64)$. The solid line represents the best fit of the experimental data using Equation 1. Standard deviations are shown as error bars.

Figure 4.8 Frequency-domain intensity decay of $40 \mu \mathrm{M}$ 1,8-ANS:54 $\mu \mathrm{M}$ $\operatorname{DREAM}(\Delta 64)$ complexes in the presence of EDTA (squares), $\mathrm{Ca}^{2+}$ (circle), $\mathrm{Cd}^{2+}$ (up triangle), and $\mathrm{Cd}^{2+} \mathrm{Ca}^{2+}$ (down triangle). Solid symbols represent phase delay, whereas empty symbols represent modulation ratio. Solid lines represent the fitting of the data using three-exponential decay model.

Figure 4.9. ITC isotherms for the displacement of $\mathrm{Ca}^{2+}$ by $\mathrm{Cd}^{2+}$ from EF-hand of (A) DREAM $(\Delta 64)$ and (B) DREAM $(\Delta 160)$. The upper panels of the profiles represent the thermal power expressed in $\mu \mathrm{cal}^{-1} \mathrm{~s}^{-1}$, whereas the lower panels show integrated reaction heats $(\Delta \mathrm{H})$ expressed in kcal. mole $^{-1}$. The solid lines represent the best fitting curve using sequential model ( 2 sites). Thermodynamics parameters recovered from the model are listed in Table 4.3.

Figure 4.10 Titration of 0.5 $\mu \mathrm{M}$ PS1HL9 against $\mathrm{Cd}^{2+}$-bound DREAM constructs. The solid lines represent the fits of the experimental data using Equation 1; standard deviations are shown as error bars.

Figure 4.11. Titration of $0.5 \mu \mathrm{M} \mathrm{Kv} 4.3$ (2-22) "site-1" against $\mathrm{Cd}^{2+}$-bound DREAM constructs. The solid lines represent the fits of the experimental data using Equation 1; standard deviations are shown as error bars.

Figure 4.12. Titration of $0.5 \mu \mathrm{M} \mathrm{Kv} 4.3$ (70-90) "site-2" against $\mathrm{Cd}^{2+}$-bound DREAM constructs. The solid lines represent the fits of the experimental data using Equation 1; standard deviations are shown as error bars.

Figure 5.1. Impact of $\mathrm{Li}^{+}$binding on tryptophan emission of (A) DREAM( $\left.\Delta 64\right)$, (B) DREAM $(\Delta 160)$, (C) DREAM(E186Q), and (D) DREAM(E234Q). In each case, $20 \mu \mathrm{M}$ protein samples in $20 \mathrm{mM}$ Tris were excited at 295 
$\mathrm{nm}$; concentrations of EDTA, $\mathrm{Ca}^{2+}$, and $\mathrm{Li}^{+}$in each DREAM construct were $2 \mathrm{mM}, 2 \mathrm{mM}$, and $50 \mu \mathrm{M}$, respectively; $\mathrm{Ca}^{2+} \mathrm{Li}+\mathrm{DREAM}$ constructs were prepared by adding $2 \mathrm{mM} \mathrm{Ca}^{2+}$ to DREAM constructs in the presence of $50 \mu \mathrm{M} \mathrm{Li}^{+}$.

Figure 5.2. Impact of $\mathrm{Li}^{+}$binding to DREAM(65-256) in the presence of 100 $\mathrm{mM} \mathrm{Na}{ }^{+}$or $100 \mathrm{mM} \mathrm{K}{ }^{+}$. Condition: $20 \mu \mathrm{M}$ DREAM(65-256), $2 \mathrm{mM}$ $\mathrm{Li}^{+}$, excited at $295 \mathrm{~nm}$.

Figure 5.3. Impact of $\mathrm{Li}^{+}$binding on far-UV CD spectra of (A) $20 \mu \mathrm{M}$ $\operatorname{DREAM}(\Delta 64)$, (B) $20 \mu \mathrm{M}$ DREAM( $\Delta 160)$, (C) $20 \mu \mathrm{M}$ DREAM(E186Q), and (D) $20 \mu \mathrm{M}$ DREAM(E234Q) in $20 \mathrm{mM}$ Tris. Concentrations of EDTA, $\mathrm{Ca}^{2+}$, and $\mathrm{Li}^{+}$in each DREAM construct were $2 \mathrm{mM}, 2 \mathrm{mM}$ and $50 \mu \mathrm{M}$, respectively; $\mathrm{Ca}^{2+} \mathrm{Li}^{+}$DREAM constructs were obtained adding $2 \mathrm{mM} \mathrm{Ca}^{2+}$ to DREAM constructs in the presence of 50 $\mu \mathrm{M} \mathrm{Li}^{+}$. All experiments were conducted at $18^{\circ} \mathrm{C}$ using a $\mathrm{J}-810 \mathrm{Jasco}$ CD spectrometer through the $2 \mathrm{~mm}$ path of a $(2 \mathrm{~mm} \times 10 \mathrm{~mm})$ quartz cuvette.

Figure 5.4. ITC isotherms for $\mathrm{Li}^{+}$binding to EF-hand of metal-free A) $\operatorname{DREAM}(\Delta 64)$ and B) DREAM $(\Delta 160) .30 \times 6 \mu \mathrm{L}$ of $1 \mathrm{mM} \mathrm{Li}^{+}$were titrated into $20 \mu \mathrm{M}$ DREAM.Contribution of buffer was corrected. Thermal power expressed in $\mu \mathrm{cal} . \mathrm{s}^{-1}$ is shown in the upper panels of the profiles, whereas integrated reaction heats $(\Delta \mathrm{H})$ expressed in kcal.mole ${ }^{-1}$ is shown in the lower panels. The solid lines correspond to the best fitting curve using sequential model with two sites; thermodynamics parameters obtained from the model are listed in Table 1.

Figure 5.5. A) Emission intensity of $20 \mu \mathrm{M}$ metal-free DREAM( $\Delta 64)$ upon $\mathrm{Li}^{+}$ addition; DREAM $(\Delta 64)$ was prepared in $20 \mathrm{mM}$ Tris, excited at $295 \mathrm{~nm}$ B) Titration curve for $\mathrm{Li}+$ binding to metal-free $\operatorname{DREAM}(\Delta 64) . \Delta \mathrm{F}$ was calculated based on decrease in Trp169 emission intensity at emission maximum. Solid line represents fit of the data using Equation 2. ......

Figure 5.6. (a) Lithium ions strongly bound to DREAM protein as revealed by the molecular simulations. One of the binding sites (right) is also a calcium binding site. b) Other transient binding sites observed during the simulations. (c) Root mean square fluctuation of backbone atoms in protein for the first $50 \mathrm{~ns}$ (black) and the last $50 \mathrm{~ns}$ (red) of simulation. Protein conformational flexibility is significantly reduced upon biding 
with lithium ions. (d) Root mean square deviation of protein with respect to time, showing a stable protein- $\mathrm{Li}^{+}$complex.

Figure 5.7. Impact of $\mathrm{Li}^{+}$binding on secondary structure of $20 \mu \mathrm{MrCaM}$ in 20 $\mathrm{mM}$ Tris (A). Consequence of $\mathrm{Li}^{+}$binding on the phenylalanine emission (B) and tyrosine emission (C) of the same protein in the same condition. Samples were excited at $250 \mathrm{~nm}$ and $277 \mathrm{~nm}$ for Figure (B) and $(\mathrm{C})$, respectively. Concentrations of EDTA, $\mathrm{Ca}^{2+}$ and $\mathrm{Li}^{+}$were 2 $\mathrm{mM}, 2 \mathrm{mM}$, and $2 \mathrm{mM}$, respectively.

Figure 5.8. Consequences of $\mathrm{Li}^{+}$binding on emission spectra of (A) 1,8ANS:DREAM $(\Delta 64)$, (B) 1,8-ANS:DREAM $(\Delta 160$ complexes. Conditions: $20 \mu \mathrm{M}$ DREAM, $20 \mu \mathrm{M}$ 1,8-ANS; excited at $350 \mathrm{~nm}$. Concentrations of EDTA, $\mathrm{Ca}^{2+}$, and $\mathrm{Li}^{+}$were same as tryptophan emission.

Figure 5.9. Frequency-domain intensity decay of $40 \mu \mathrm{M}$ 1,8-ANS:54 $\mu \mathrm{M}$ $\operatorname{DREAM}(\Delta 64)$ complexes in $20 \mathrm{mM}$ Tris and in the presence of $2 \mathrm{mM}$ EDTA (squares), $2 \mathrm{mM} \mathrm{Ca}^{2+}$ (circle), $50 \mu \mathrm{M} \mathrm{Li}^{+}$(up triangle), and (2 $\mathrm{mM} \mathrm{Ca}{ }^{2+}$ and $50 \mu \mathrm{M} \mathrm{Li}^{+}$) $\mathrm{Ca}^{2+} \mathrm{Li}^{+}$(down triangle). Phase delay and modulation ratio are respectively shown as solid and empty symbols. Solid lines correspond to the fitting of the data using three-exponential decay model.

Figure 5.10. Impact of $\mathrm{Li}^{+}$association with DREAM on DREAM interactions with interacting partners. $100 \mu \mathrm{M}, 200 \mu \mathrm{M}$, and $100 \mu \mathrm{M} \operatorname{DREAM}(\Delta 64)$ were respectively added to FITC-labeled A) $0.5 \mu \mathrm{M} \mathrm{K}_{v} 4.3-(2-22)$. B) $0.5 \mu \mathrm{M} \mathrm{K}_{\mathrm{v}} 4.3-(70-90)$, and C) $0.5 \mu \mathrm{M}$ PS1HL9. Samples were excited at $470 \mathrm{~nm}$; emission was recorded through $500 \mathrm{~nm}$ long-pass filter. Concentrations of EDTA, $\mathrm{Ca}^{2+}$, and $\mathrm{Li}^{+}$were same as Trp emission study.

Figure 6.1. Impact of $1 \mathrm{mM} \mathrm{Mn}^{2+}$ addition on Trp emission of metal-free and $\mathrm{Ca}^{2+}$-bound DREAM $(\Delta 64)(\mathrm{A}, \mathrm{B})$ and emission of metal free and $\mathrm{Ca}^{2+}$ bound DREAM $(\Delta 64): 1,8-A N S(C, D)$.

Figure 6.2. Impact of $1 \mathrm{mM} \mathrm{As}^{5+}$ addition on Trp emission of metal-free and $\mathrm{Ca}^{2+}$-bound DREAM $(\Delta 64)(\mathrm{A}, \mathrm{B})$ and emission of metal free and $\mathrm{Ca}^{2+}$ bound DREAM $(\Delta 64): 1,8-A N S(C, D)$. 
Figure 6.3. Impact of $1 \mathrm{mM} \mathrm{Hg}^{2+}$ addition on Trp emission of metal-free and $\mathrm{Ca}^{2+}$-bound DREAM $(\Delta 64)(\mathrm{A}, \mathrm{B})$ and emission of metal free and $\mathrm{Ca}^{2+}$ bound $\operatorname{DREAM}(\Delta 64): 1,8-\mathrm{ANS}(\mathrm{C}, \mathrm{D})$.

Figure 6.4. Impact of $1 \mathrm{mM} \mathrm{Ba}^{2+}$ addition on Trp emission of metal-free and $\mathrm{Ca}^{2+}$-bound DREAM $(\Delta 64)(\mathrm{A}, \mathrm{B})$ and emission of metal free and $\mathrm{Ca}^{2+}$ bound $\operatorname{DREAM}(\Delta 64): 1,8-\mathrm{ANS}(\mathrm{C}, \mathrm{D})$.

Figure 6.5. Impact of $1 \mathrm{mM} \mathrm{Sr}^{2+}$ addition on Trp emission of metal-free and $\mathrm{Ca}^{2+}$-bound DREAM $(\Delta 64)(\mathrm{A}, \mathrm{B})$ and emission of metal free and $\mathrm{Ca}^{2+}$ bound $\operatorname{DREAM}(\Delta 64): 1,8-\mathrm{ANS}(\mathrm{C}, \mathrm{D})$.

Figure 7.1. Left: aMD structure of $\mathrm{Ca}^{2+} \mathrm{DREAM}$. EF-hands are shown in different colors: EF-hand 1, yellow; EF-hand 2, blue; EF-hand 3, green; EF-hand 4, red; rest part of the protein is shown in cyan; two $\mathrm{Ca}^{2+}$ are shown as green spheres; Trp169, Glu165, and Lys87 are show as sticks. Right: Distance between Lys87 and Glu165 side chains determined from $100 \mathrm{~ns}$ accelerated molecular simulation of $\operatorname{DREAM}(\Delta 64)$.

Figure 7.2. (A) Intrinsic fluorescence of $20 \mu \mathrm{M}$ DREAM(K87A) in the presence of $1 \mathrm{mM}$ EDTA, $5 \mathrm{mM} \mathrm{Mg}^{2+}$, and $1 \mathrm{mM} \mathrm{Ca}^{2+}$; excited at $295 \mathrm{~nm}$. (B) Far-UV CD spectra of DREAM(K87A) under identical condition. ......

Figure 7.3. Fluorescence emission of DREAM(K87A):1,8-ANS complex. Condition: $20 \mu \mathrm{M}$ DREAM(K87A), $20 \mu \mathrm{M}$ 1,8-ANS, excited at $350 \mathrm{~nm}$.

Figure 7.4. Frequency-domain intensity decay of $20 \mu \mathrm{M}$ DREAM(K87A) in apo (square), $\mathrm{Mg}^{2+}$ (circle), $\mathrm{Ca}^{2+}$ (upper triangle), and $\mathrm{Ca}^{2+} \mathrm{Mg}^{2+}$ (lower triangle) bound form. Solid symbols correspond to phase delay; empty symbols correspond to modulation ratio. Solid lines represent data fitting using a sum of three exponential decays model.

152

Figure 7.5. Frequency-domain intensity decay of DREAM(K87A):1,8-ANS in apo (square), $\mathrm{Mg}^{2+}$ (circle), $\mathrm{Ca}^{2+}$ (upper triangle), and $\mathrm{Ca}^{2+} \mathrm{Mg}^{2+}$ (lower triangle) bound form. Solid symbols correspond to phase delay; empty 
symbols correspond to modulation ratio. Solid lines represent data fitting using a sum of three exponential decays model.

Figure 7.6. Anisotropy change associated with Kv4.3 site 1 (residues 2-22) (A), Kv4.3 site 2 (residues 70-90) (B) and presenilin 1 helix 9 (PS1HL9) (C) binding to DREAM(K87A) in the presence and absence of $\mathrm{Ca}^{2+}$.

Figure 8.1. Steady-state Trp emission spectra of $20 \mu \mathrm{M} \operatorname{DREAM}(\Delta 64)$, $\operatorname{DREAM}(\Delta 160), \operatorname{DREAM}(\mathrm{E} 186 \mathrm{Q})$, and DREAM(E234Q) in the absence and presence of $1 \mathrm{mM} \mathrm{Ca}^{2+}$; excited at $295 \mathrm{~nm}$.

Figure 8.2. Frequency-domain intensity decay of $20 \mu \mathrm{M}$ DREAM(E186Q) (A) and DREAM(E234Q) (B) in apo (square) and $\mathrm{Ca}^{2+}$ (circle) bound form. Solid symbols represent phase delay; empty symbols represent modulation ratio. Solid lines represent fitting of the data using a sum of three exponential decays model.

Figure 8.3. Far-UV CD spectra of DREAM( $\Delta 64)(A), \operatorname{DREAM}(\Delta 160)(B)$, $\operatorname{DREAM}(\mathrm{E} 186 \mathrm{Q})(\mathrm{C})$, and DREAM(E234Q) (D) in the absence and presence of $1 \mathrm{mM} \mathrm{Ca}{ }^{2+}$.

Figure 8.4. Fluorescence emission of DREAM $(\Delta 64)$ :1,8-ANS (A), $\operatorname{DREAM}(\Delta 160): 1,8$-ANS (B), DREAM(E186):1,8-ANS (C), and DREAM(E234):1,8-ANS complexes in metal-free form and in the presence of $1 \mathrm{mM} \mathrm{Ca}^{2+}$. Condition: $20 \mu \mathrm{M}$ DREAM constructs, $20 \mu \mathrm{M}$ 1,8-ANS, excited at $350 \mathrm{~nm}$.

Figure 8.5. Frequency-domain intensity decay of DREAM(E186Q):1,8-ANS (A) and DREAM(E234Q):1,8-ANS (B) in apo (square) and $\mathrm{Ca}^{2+}$-bound form (circle). Solid symbols represent phase delay; empty symbols represent modulation ratio. Solid lines represent fitting of the data using a sum of three exponential decays model.

Figure 8.6. Anisotropy change associated with Kv4.3 site-1 (residues 2-22) (A) and Kv4.3 site- 2 (residues 70-90) (B) association with $\operatorname{DREAM}(\mathrm{E} 186 \mathrm{Q})$ and DREAM(E234Q) in the presence and absence of $\mathrm{Ca}^{2+}$. 


\section{LIST OF ABBREVIATIONS AND ACRONYMS}

\begin{tabular}{|c|c|}
\hline ABBREVIATION & FULL NAME \\
\hline 1,8 -ANS & 1-anilinonaphthalene-8-sulfonic acid \\
\hline aMD & Accelerated molecular dynamics \\
\hline $\mathrm{Ca}^{2+}$ & Calcium ion \\
\hline $\mathrm{CaBP}$ & Calcium binding protein \\
\hline $\mathrm{CaM}$ & Rat calmodulin \\
\hline $\mathrm{cMD}$ & Classical molecular dynamics \\
\hline $\mathrm{Cd}^{2+}$ & Cadmium ion \\
\hline $\mathrm{CD}$ & Circular dichroism \\
\hline DREAM & Downstream regulatory element antagonist modulator \\
\hline EDTA & $\begin{array}{l}\text { 2-(\{2-[Bis(carboxymethyl)amino]ethyl }\} \\
\text { (carboxymethyl)amino)acetic acid }\end{array}$ \\
\hline EFX & EF-hand X \\
\hline FITC & Fluorescein isothiocyanate \\
\hline ITC & Isothermal titration calorimetry \\
\hline IMPase & Inositol monophosphatase \\
\hline IPTG & Isopropyl $\beta$-D-1-thiogalactopyranoside \\
\hline $\mathrm{InsP}_{3} \mathrm{R}$ & Inositol triphosphate receptors \\
\hline KChIP & Potassium channel interaction protein \\
\hline$\lambda_{\max }$ & Emission maxima \\
\hline $\mathrm{Li}^{+}$ & Lithium ion \\
\hline LTD & Long-term depression \\
\hline
\end{tabular}




$\begin{array}{ll}\mathrm{Mg}^{2+} & \text { Magnesium ion } \\ \text { NAIP } & \text { Neuronal apoptosis inhibitor protein } \\ \mathrm{NCS} & \text { Neuronal calcium sensor } \\ \mathrm{Pb}^{2+} & \text { Lead ion } \\ \text { PKC } & \text { Protein Kinase C } \\ \text { POPOP } & \text { 1,4-bis(5-phenyloxazol-2-yl)-benzene } \\ \text { PS1-CTF } & \text { Presenilin-1 carboxy-terminal fragment } \\ \text { PS1HL9 } & \text { Helix-9 of presenilin-1 } \\ \text { ROCs } & \text { Receptor-operated ion channels } \\ \text { RMSD } & \text { Root mean square deviation } \\ \text { RYR } & \text { Ryanodine receptors } \\ \text { SASA } & \text { Solvent accessible surface area } \\ \text { SMOCs } & \text { Secondary messenger operated Ca }{ }^{2+} \text { channels }\end{array}$




\section{PREFACE}

This dissertation is an accumulation of research studies conducted by Samiol Azam from August 2015 to June 2019. During this time, chapter 3 (Azam, Miksovska 2018) and 4 (Azam, St Luis et al. 2019) have been published in the peer-reviewed journals ACS Chemical Neuroscience and Metallomics, respectively. Chapter 5 has been submitted to ACS Chemical Neuroscience for publication. 


\section{INTRODUCTION}

\subsection{General principle of calcium signaling}

Calcium $\left(\mathrm{Ca}^{2+}\right)$ is a universal intracellular signaling ion that binds to a wide spectrum of calcium-binding proteins $(\mathrm{CaBPs})$ and regulate numerous cellular functions, including muscle contraction, fertilization, gene transcription, exocytosis, energy metabolism, neurotransmitter secretion, activity of ion channels, and signal transduction (Gifford, Walsh et al. 2007, Berridge, Lipp et al. 2000, Carafoli, Santella et al. 2001). Dysregulation of calcium signaling could result in cancer, manic depression, hypertension, heart disease, and Alzheimer's disease (Gifford, Walsh et al. 2007, Berridge, Bootman et al. 2003). In the resting cells, intracellular $\mathrm{Ca}^{2+}$ concentration is about $100 \mathrm{nM}$, while concertation of extracellular $\mathrm{Ca}^{2+}$ is about $2 \mathrm{mM}$ (Vogel 1994, Clapham 2007). Such an enormous difference between intracellular $\mathrm{Ca}^{2+}$ and extracellular $\mathrm{Ca}^{2+}(10,000$ fold $)$ generates a chemiosmosis force that facilitates the influx of $\mathrm{Ca}^{2+}$ into the cell. The ATPdependent pump maintains intracellular $\mathrm{Ca}^{2+}$ concentration by pumping excess $\mathrm{Ca}^{2+}$ out of the cells or into internal stores such as mitochondria, endoplasmic and sarcoplasmic reticulum (Gifford, Walsh et al. 2007, Clapham 2007). Intracellular calcium binds to a wide array of $\mathrm{CaBPs}$ that regulate a wide range of cellular processes. Consequently, the $\mathrm{Ca}^{2+}$ signal is translated into diverse biochemical responses by triggering different intracellular pathways (Carafoli, Santella et al. 2001, Berridge, Bootman et al. 2003, Clapham 2007).

The regulation of a wide range of cellular processes by a single $\mathrm{Ca}^{2+}$ ion emphasizes the versatility of this ion in cellular function (Berridge, Lipp et al. 2000). The question remains how $\mathrm{Ca}^{2+}$ regulates so many cellular processes? The versatility of calcium signaling could be explained by its wide range of spatial and temporal signals, speed and 
amplitude (Berridge, Lipp et al. 2000). The versatility is achieved through the use of an extensive $\mathrm{Ca}^{2+}$ signaling toolkit. The calcium-signaling toolkit comprises voltage-operated channels, receptor-operated channels, calcium pumps and exchangers, calcium buffer proteins, $\mathrm{Ca}^{2+}$ effector proteins, receptor proteins, transducer proteins, transcriptional factors, endoplasmic reticulum (ER), sarcoplasmic reticulum (SR), Golgi apparatus, mitochondria, to name a few (Berridge, Lipp et al. 2000, Berridge, Bootman et al. 2003). Each cell type utilizes distinctive components from the $\mathrm{Ca}^{2+}$-signaling toolkit and generates $\mathrm{Ca}^{2+}$ signals with widely different spatial and temporal properties (Berridge, Bootman et al. 2003). Thanks to the flexibility of $\mathrm{Ca}^{2+}$ as a ligand. Although $\mathrm{Ca}^{2+}$ has similar physical properties as $\mathrm{Mg}^{2+}, \mathrm{Ca}^{2+}$ binding sites have significantly higher affinity for $\mathrm{Ca}^{2+}$ than $\mathrm{Mg}^{2+}$, as the coordination chemistry of $\mathrm{Ca}^{2+}$ allows it to bind to the binding site of irregular geometry whereas the strict requirement of $\mathrm{Mg}^{2+}$ for octahedral geometry is hard to satisfy

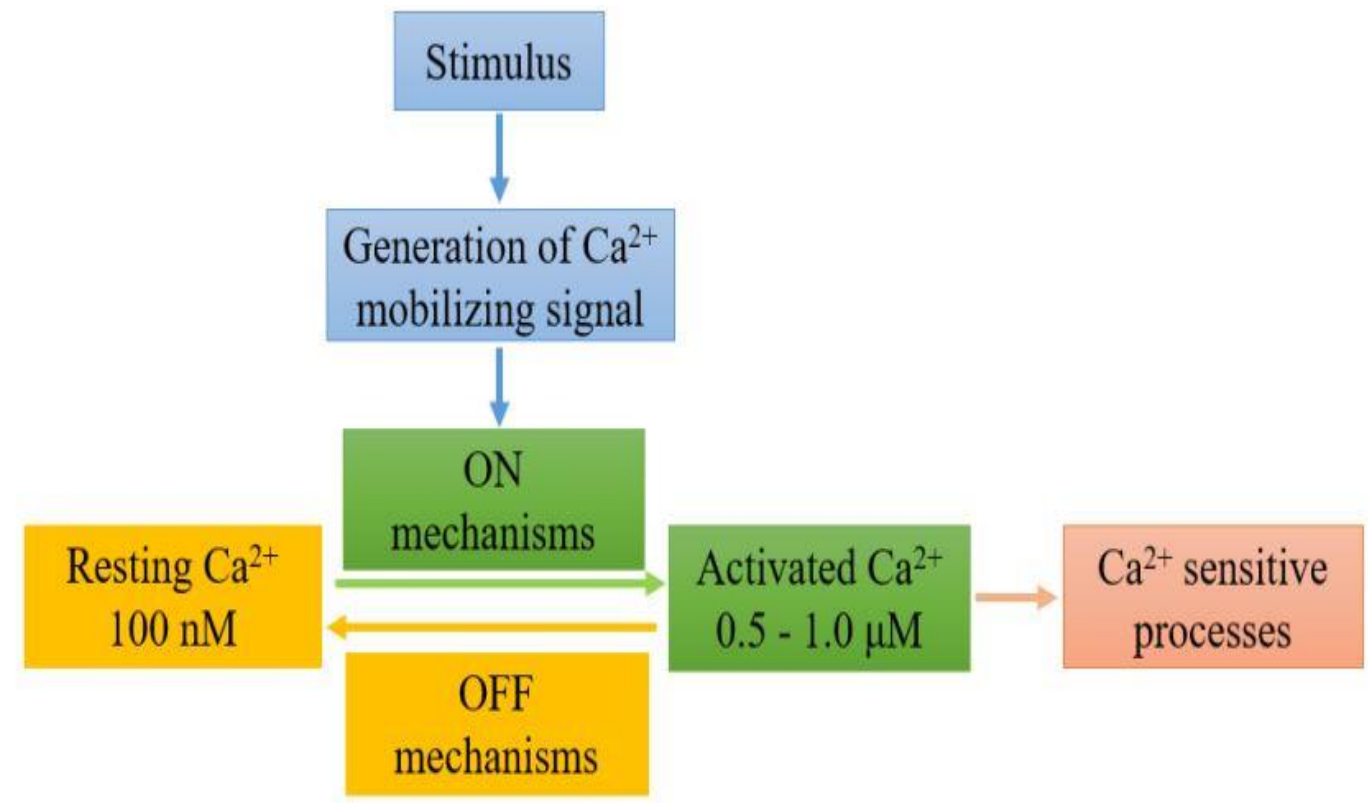

Figure 1.1. Components of $\mathrm{Ca}^{2+}$ signaling network. Maintenance of intracellular $\mathrm{Ca}^{2+}$ concentration is determined by the balance between ON/OFF reaction. Adapted from (Berridge, Lipp et al. 2000). 
within the structure of proteins (Brini, Calì et al. 2014). A common calcium signaling network comprises four functional units, where at any moment, the level of intracellular $\mathrm{Ca}^{2+}$ is determined by the balance between ON/OFF reaction as shown in Figure 1.1 (Berridge, Lipp et al. 2000). Calcium signaling is initiated by stimuli such as membrane depolarization, extracellular signaling molecules, intracellular messengers, stretch, and noxious stimuli, which generates $\mathrm{Ca}^{2+}$ mobilizing signals (Berridge, Bootman et al. 2003). Calcium mobilizing signals activate the $\mathrm{ON}$ mechanism and extracellular $\mathrm{Ca}^{2+}$ enter the cytoplasm through different channels, including voltage-operated ion channels (VOCs), store-operated $\mathrm{Ca}^{2+}$ channels (SOCs), receptor-operated ion channels (ROCs), and secondary messenger operated $\mathrm{Ca}^{2+}$ channels (SMOCs) (Gifford, Walsh et al. 2007, Berridge, Bootman et al. 2003). Additionally, internal storages such as the endoplasmic reticulum (ER) and sarcoplasmic reticulum (SR) can also release $\mathrm{Ca}^{2+}$ into the cytoplasm. The release of $\mathrm{Ca}^{2+}$ from internal stores is controlled by different channels, including ryanodine receptors (RYR) and inositol triphosphate receptors ( $\left.\operatorname{InsP}_{3} \mathrm{R}\right)$. Calcium itself is the activator of these channels. Activation of those channels by $\mathrm{Ca}^{2+}$ is dependent on whether stimuli bind to cell surface receptors. For instance, inositol triphosphate ( $\left.\operatorname{InsP}_{3}\right)$ diffuses into the cells to bind inositol triphosphate receptors $\left(\operatorname{InsP}_{3} \mathrm{R}\right)$ and release $\mathrm{Ca}^{2+}$ from ER (Gifford, Walsh et al. 2007, Berridge, Lipp et al. 2000). The $\mathrm{Ca}^{2+}$ that enters the cytoplasm during the ON mechanism does not remain unbound. Instead, it interacts with different $\mathrm{CaBPs}$. Most of cytoplasmic $\mathrm{Ca}^{2+}$ rapidly binds to calcium buffer proteins, whereas a small proportion of it binds to numerous $\mathrm{Ca}^{2+}$ sensor proteins, including calmodulin $(\mathrm{CaM})$, recoverin, troponin, and neuronal $\mathrm{Ca}^{2+}$ sensor proteins (NCS) in the brain (Gifford, Walsh et al. 2007, Berridge, Bootman et al. 2003). These calcium sensors 
transduce chemical signals of an increased $\mathrm{Ca}^{2+}$ concentration into biochemical responses by triggering diverse signaling pathways (Gifford, Walsh et al. 2007). At last, once $\mathrm{Ca}^{2+}$ has completed its signaling functions, it is rapidly removed from the cytoplasm with different exchangers and pumps via OFF mechanism. $\mathrm{Na}^{+} / \mathrm{Ca}^{2+}$ exchangers and plasma membrane $\mathrm{Ca}^{2+}$-ATPase (PMCA) pumps extrude $\mathrm{Ca}^{2+}$ outside of the cells. Other ion channels, including sarco-endoplasmic calcium ATPases (SERCAs), $\mathrm{H}^{+} / \mathrm{Ca}^{2+}$ exchangers, and $\mathrm{Ca}^{2+}$ uniporters return $\mathrm{Ca}^{2+}$ into the internal stores, such ER, SR, and mitochondria (Gifford, Walsh et al. 2007, Berridge, Lipp et al. 2000).

\subsection{Neuronal calcium signaling}

Calcium plays an important and diversified role in neuronal physiology. For instance, $\mathrm{Ca}^{2+}$ impacts both long-term depression (LTD) and long-term potentiation forms of synaptic plasticity by controlling the release of neurotransmitter from the presynaptic terminal (Bolshakov, Siegelbaum 1994, Christie, Magee et al. 1996, Grover, Teyler 1990, Impey, Mark et al. 1996). As a universal secondary messenger, $\mathrm{Ca}^{2+}$ has been demonstrated to regulate the process of learning and the formation and consolidation of memory, synaptogenesis, membrane excitability, and dendrite development (Brini, Calì et al. 2014, Südhof 2004, Redmond, Ghosh 2005, Michaelsen, Lohmann 2010, Tanaka, Nakada et al. 2008). The intracellular concentration of neuronal cell is in the range $100-500 \mathrm{nM}$, whereas extracellular concentration is $1 \mathrm{mM}$ (Kawamoto, Vivar et al. 2012). Like other cells, neuronal cells use different components from the $\mathrm{Ca}^{2+}$ signaling toolkit to generate neuronal $\mathrm{Ca}^{2+}$ signals with a wide range of temporal and spatial properties. Neuronal life is dependent on the correct functioning of the above processes. Since $\mathrm{Ca}^{2+}$ plays a prominent role in the neuronal function, subtle alteration in $\mathrm{Ca}^{2+}$ homeostasis leads to 
profound functional changes (Brini, Calì et al. 2014, Kawamoto, Vivar et al. 2012). Several lines of evidence have demonstrated that perturbation of $\mathrm{Ca}^{2+}$ homeostasis is a causative factors of amyotrophic lateral sclerosis (ALS), Alzheimer's, Parkinson's, Huntington disease, and other neurodegenerative disease (Christie, Magee et al. 1996, Kawamoto, Vivar et al. 2012, Bezprozvanny 2009, Bezprozvanny 2007, Mattson 2007, Mattson 2004, Rowland, Shneider 2001, von Lewinski, Keller 2005, Lim, Fedrizzi et al. 2008). Alteration of $\mathrm{Ca}^{2+}$ homeostasis could be attributed to the decrease in $\mathrm{Ca}^{2+}$ buffering capacity of $\mathrm{Ca}^{2+}-$ buffer proteins, malfunction of membrane channels and mitochondrial $\mathrm{Ca}^{2+}$ controlling system (Brini, Calì et al. 2014, Kawamoto, Vivar et al. 2012).

\subsection{Classification of $\mathrm{Ca}^{2+}$ binding proteins}

As it is mentioned earlier, $\mathrm{Ca}^{2+}$ that flows into the cell during the $\mathrm{ON}$ reaction interacts with numerous CaBPs; the human genome encodes more than 200 CaBPs(Berridge, Bootman et al. 2003). Functionally, CaBPs are divided into two classes: $\mathrm{Ca}^{2+}$ buffers and $\mathrm{Ca}^{2+}$ sensors (Brini, Calì et al. 2014). Calcium buffers bind $\mathrm{Ca}^{2+}$ with high affinity but $\mathrm{Ca}^{2+}$ association does not lead significant conformational changes. Calcium buffers proteins modulate $\mathrm{Ca}^{2+}$ signaling and help maintain $\mathrm{Ca}^{2+}$ homeostasis by releasing $\mathrm{Ca}^{2+}$ during the $\mathrm{ON}$ reaction and sequestering $\mathrm{Ca}^{2+}$ during OFF reaction (Gifford, Walsh et al. 2007, Berridge, Bootman et al. 2003). Another important purpose of $\mathrm{Ca}^{2+}$ buffer is to regulate the transmission of $\mathrm{Ca}^{2+}$ signaling throughout the cells by limiting the amplitude and duration of $\mathrm{Ca}^{2+}$ signaling (Braunewell, Gundelfinger 1999). Parvalbumin, calbindin D-9k, calbindin D-28k, and calretinin are few examples of $\mathrm{Ca}^{2+}$ buffer proteins (Gifford, Walsh et al. 2007, Berridge, Bootman et al. 2003). On the other hand, $\mathrm{Ca}^{2+}$ sensors proteins undergo significant conformational changes upon $\mathrm{Ca}^{2+}$ association, which enables them to 
interact with binding partners to regulate a plethora of $\mathrm{Ca}^{2+}$-dependent processes (Gifford, Walsh et al. 2007, Braunewell, Gundelfinger 1999). Recoverin, calmodulin, troponin C, calcineurin $\mathrm{B}$, and myosin light chain are well-studied examples of $\mathrm{Ca}^{2+}$ sensing proteins (McCue, Haynes et al. 2010). Some proteins have both $\mathrm{Ca}^{2+}$ buffering and sensing capability; CaM is one classic example of such proteins (Brini, Calì et al. 2014). All CaBPs comprise one of the three types of $\mathrm{Ca}^{2+}$ binding structural motifs: the $\mathrm{C} 2$-domain, the annexin domain and the EF-hand domain.

\section{4 $\mathrm{Ca}^{2+}$ binding motif: $\mathrm{C}-2$ domain.}

The C2-domain is a conserved functional domain that is composed of approximately 130 amino acid residues. Structural studies demonstrated that C2-domain comprises eight $\beta$ strands; 4 pairs of antiparallel $\beta$ strands are connected through flexible loops as shown Figure 1.2 (Essen, Perisic et al. 1996, Nalefski, Falke 1996). These flexible

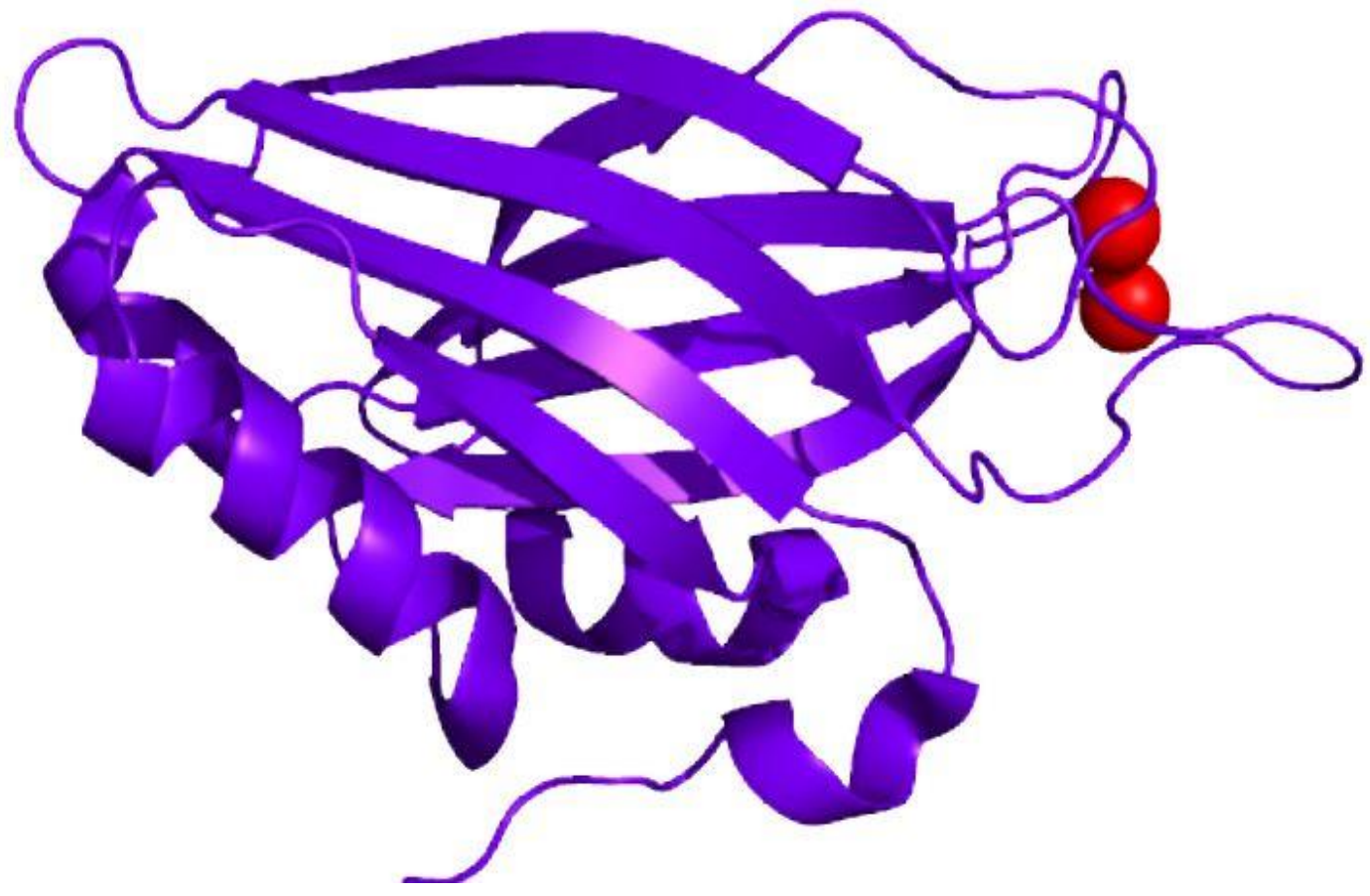

Figure 1.2. Three-dimensional structure of synaptotagmin-1 with $\mathrm{Ca}^{2+}$ bound to $\mathrm{C} 2 \mathrm{~B}$ domain (PDB: 1tjx). Cartoon representation of protein is shown as purple blue; $\mathrm{Ca}^{2+}$ is shown as red spheres. 
loops provide Asp residues for $\mathrm{Ca}^{2+}$ coordination. Each $\mathrm{Ca}^{2+}$ is hepta-coordinated in $\mathrm{C} 2-$ domain; five of the coordinating ligands come from Asp residue of the flexible loop and two of them are supplied by the water molecule (Nalefski, Falke 1996). It has been shown $\mathrm{Ca}^{2+}$ binding to $\mathrm{C} 2$-domain facilitates $\mathrm{C} 2$ domain-containing protein interaction with phospholipid membranes and other proteins (Scott, White et al. 1990, Newton 1995). Modulation of interaction between C- 2 domain protein and binding partners by $\mathrm{Ca}^{2+}$ can be explained by the exposure of hydrophobic residues as well as exposure of charged residues which can form electrostatic interaction with phosphate groups of phospholipid membranes (Scott, White et al. 1990, Newton 1995).

\section{5 $\mathrm{Ca}^{2+}$ binding motif: annexin.}

The annexin family of proteins possess a highly variable N-terminal region, conserved core domain and C-terminal region (Figure 1.3) (Ortlund, Chai et al. 2004). The core domain contains a characteristics annexin repeat formed by a 70-residue-long

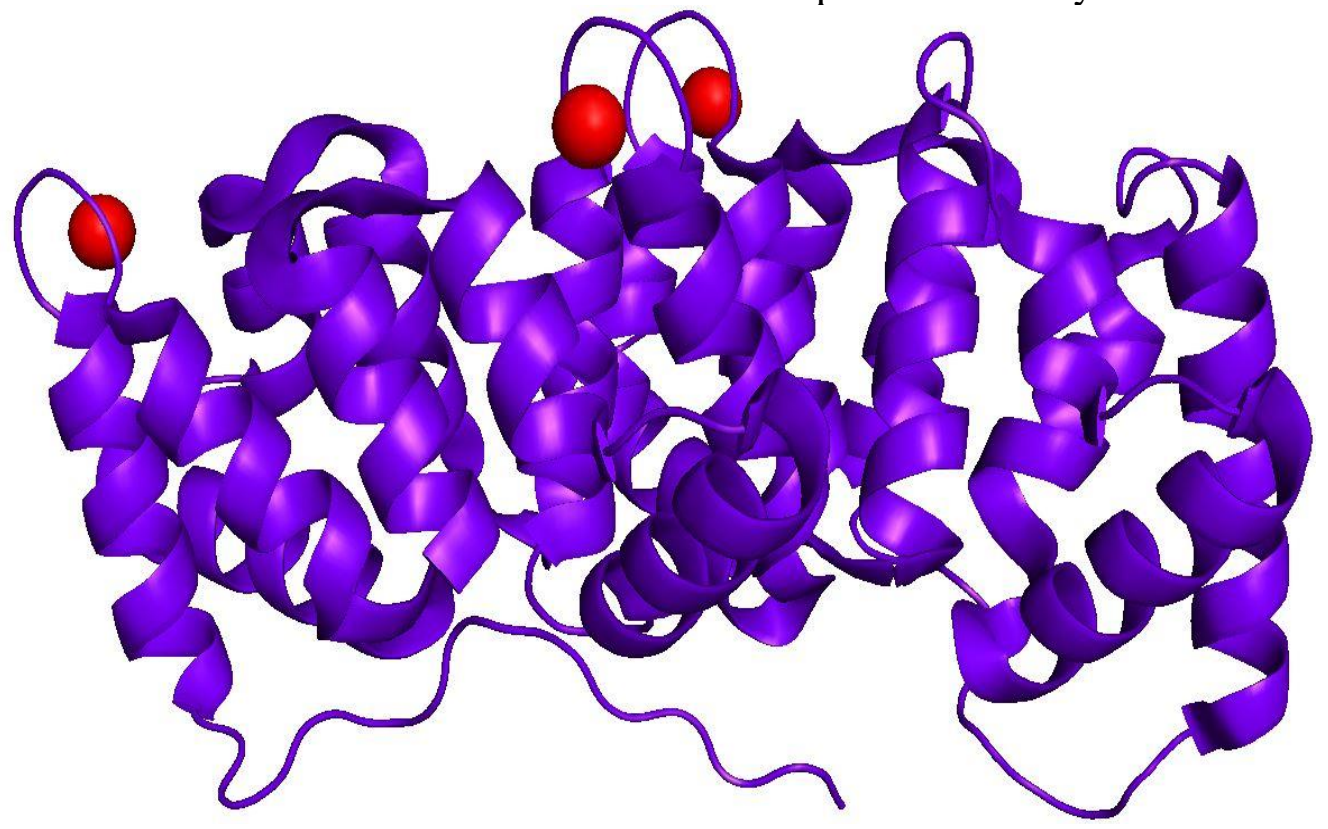

Figure 1.3. Three-dimensional structure of $\mathrm{Ca}^{2+}$ bound annexin V (PDB: 1yii). Protein is represented with purple blue cartoon; $\mathrm{Ca}^{2+}$ is represented with red spheres. 
segment. Typically, four of such annexin repeats are present in the core domain of annexin proteins. Because of the compact packing of the central core, the overall structure of the protein looks like a disc with a slight curvature forming a convex and concave surface on the top and bottom of the protein surface, respectively (Ortlund, Chai et al. 2004, Weng, Luecke et al. 1993). Core domain concave side comprises the $\mathrm{Ca}^{2+}$ binding site. Annexin has been shown to interact with phospholipid membranes in a $\mathrm{Ca}^{2+}$-dependent manner (Weng, Luecke et al. 1993). Annexin interacts with the phospholipid membrane as follows: convex surface faces towards the membrane and concave surface points away from the membrane (Weng, Luecke et al. 1993). In the apo-form of the protein, the N-terminal domain has an irregular structure that integrates into the central domain. $\mathrm{Ca}^{2+}$ association exposes the $\mathrm{N}$-terminal domain and facilitates annexin interaction with other proteins, including S100 proteins (Weng, Luecke et al. 1993). Annexins have been implicated in the regulation of different biological processes, such as endocytosis, exocytosis, membrane organization, and signal transduction (Rescher, Gerke 2004, Gerke, Moss 2002, Moss, Morgan 2004, McNeil, Rescher et al. 2006).

\section{6 $\mathrm{Ca}^{2+}$ binding motif: the EF-hand.}

The EF-hand is by far mostly used $\mathrm{Ca}^{2+}$ binding motif, having more than 250 proteins in the superfamily (Braunewell, Gundelfinger 1999). The EF-hand $\mathrm{Ca}^{2+}$ binding motif was first observed in the $\mathrm{Ca}^{2+}$ buffer protein parvalbumin (Kretsinger, Nockolds 1973, Moews, Kretsinger 1975). The name EF-hand was proposed on the basis of the observation that $\mathrm{E}$ and $\mathrm{F}$ helices of parvalbumin are oriented around the $\mathrm{Ca}^{2+}$ binding loop in such a way that resembles the thumb and index finger of a hand. After the first discovery on EF-hand motif in parvalbumin, this motif was found in troponin C (Collins, Potter et al. 


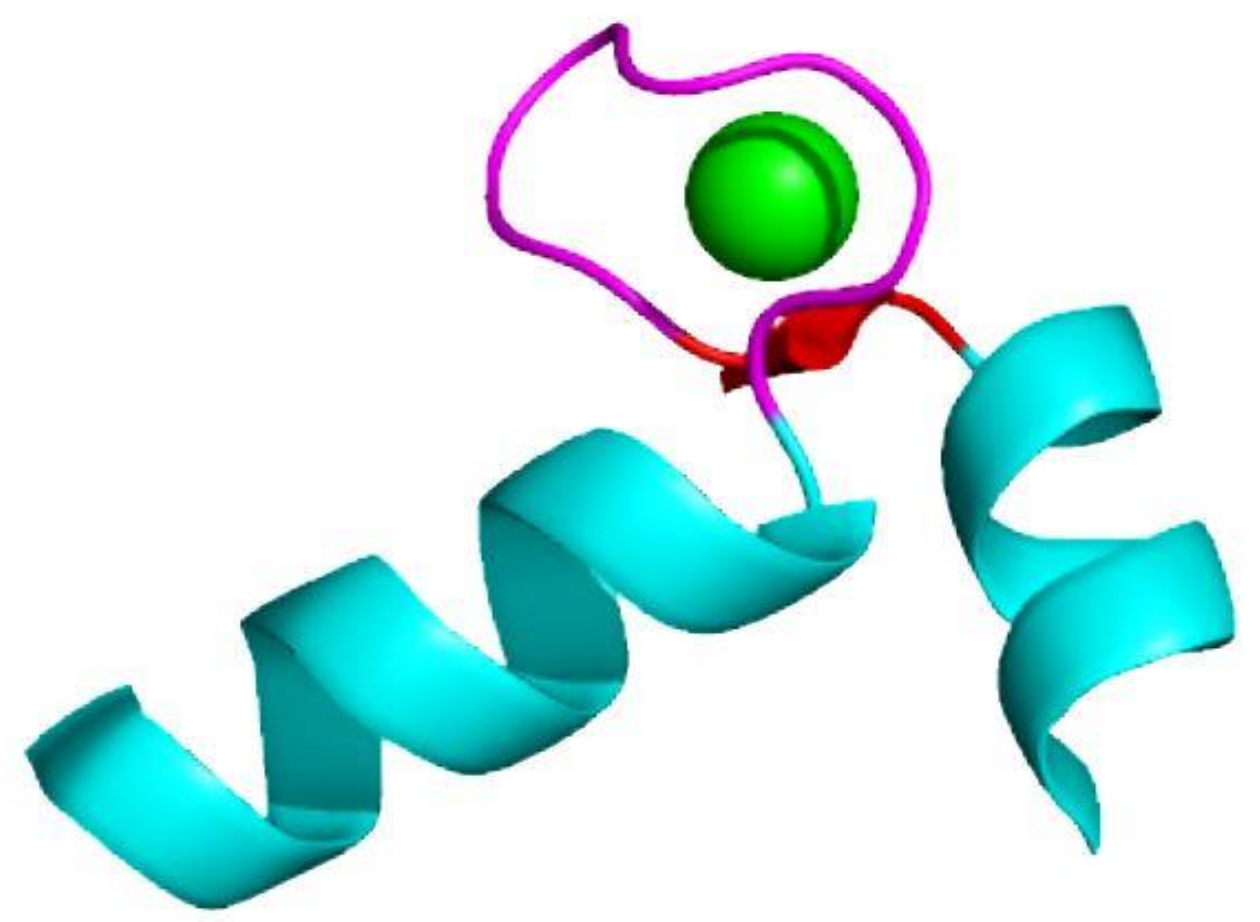

Figure 1.4. Single canonical EF-hand of parvalbumin (PDB: 1b8r). Entering and exiting helices are shown as cyan; $\mathrm{Ca}^{2+}$ chelating loop is shown as magenta; $\mathrm{Ca}^{2+}$ ion is shown as green sphere, $\beta$-sheet is shown as red.

1973), myosin light chains (Collins 1974), calmodulin (Stevens, Walsh et al. 1976), and recoverin (Flaherty, Zozulya et al. 1993), to mention a few. Most protein contains even number of EF-hands, with number four being the most prevalent. The canonical EF-hand motif comprises a helix-loop-helix structural unit, where a 9-residue entering $\alpha$-helix and an 11-residue exiting $\alpha$-helix is bridged by a 9-residue $\mathrm{Ca}^{2+}$ chelating loop (Figure 1.4) (Gifford, Walsh et al. 2007). $\mathrm{Ca}^{2+}$ is hepta-coordinated in most EF-hand protein with pentagonal bipyramidal geometry (Grabarek 2006). Because of the geometric and space constrain helices and sheets provide only few ligands for $\mathrm{Ca}^{2+}$ coordination. The 9-residue long flexible loop provides five oxygen ligands for $\mathrm{Ca}^{2+}$ coordination; the rest two oxygen ligands come from the side chain of a bidentate carboxylate ligand glutamic acid, which is 
located in the exiting helix (Gifford, Walsh et al. 2007). The coordination sphere of the canonical EF-hand loop is shown in Figure 1.5. Most of the residues in the EF-hand loop have individual roles for maintaining stability and folding of the loop: The first residue defines stereochemical rearrangement of the loop through various intraloop hydrogen

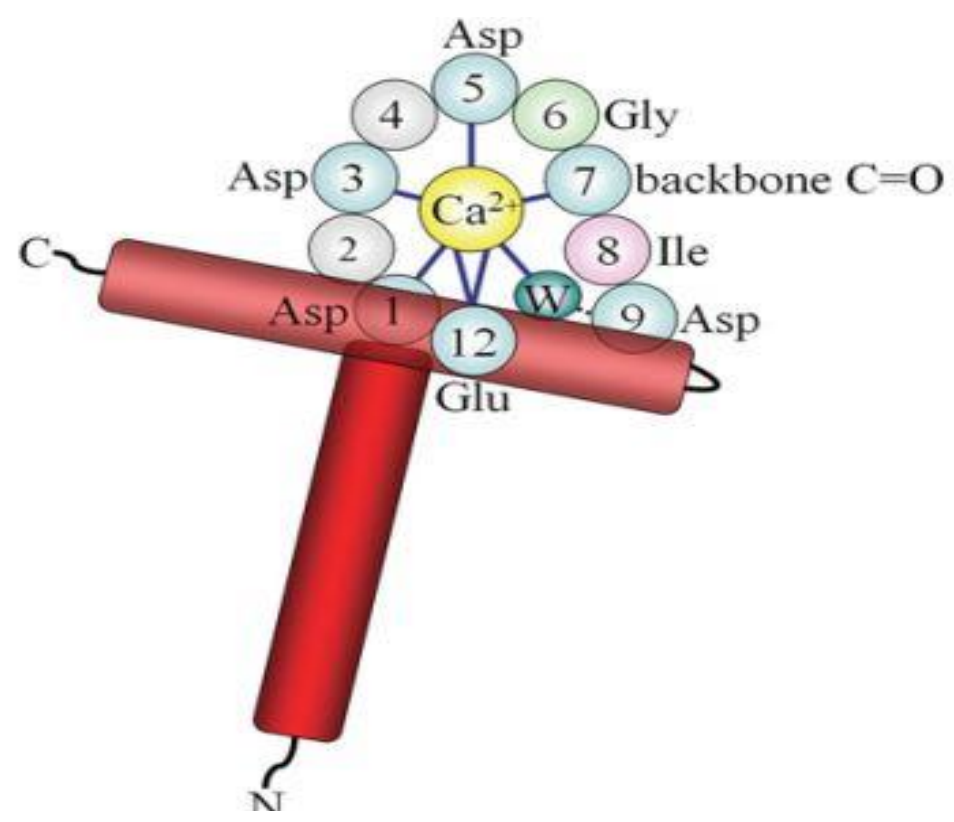

Figure 1.5. Schematic diagram of the coordination sphere of canonical EF-hand loop. Blue represents coordinating ligands, coordinating water molecule is shown in dark blue. Light green represents conserved glycine residue which allows bending of the loop. Entering and exiting helices are shown in red. Figure adapted from (Gifford, Walsh et al. 2007).

bonds. The oxygen atoms of the side chains of Asp residues at positions 1, 3, 5, and 9 and backbone carbonyl group at position 7 provide five ligands for $\mathrm{Ca}^{2+}$ coordination. Less sterically hindered amino acid residue glycine is conserved in the $6^{\text {th }}$ position, which allows $90^{\circ}$ turn of the loop that enables remaining oxygen atoms to take coordinating positions. Hydrophobic amino acid residues are conserved in the $8^{\text {th }}$ position; main chain $\mathrm{NH}$ and $\mathrm{CO}$ groups face away from $\mathrm{Ca}^{2+}$ binding site and form a short antiparallel $\beta$-sheet in this position. Aspartate residue in position 9 of the EF-hand loop can coordinate $\mathrm{Ca}^{2+}$ both 
directly and through bridging water. Glutamic acid, supplied by the C-terminal exiting helix, is present at the $12^{\text {th }}$ position of the canonical EF-hand loops and provides the remaining two oxygen atoms for $\mathrm{Ca}^{2+}$ coordination (Gifford, Walsh et al. 2007). The location of the amino acid residue at the $12^{\text {th }}$ position of the loop is critical as it determines the structure and function of the loop and specificity of the EF-hand. For instance, when glutamic acid is present in the $12^{\text {th }}$ position, it can donate two ligands to fulfill the heptacoordination of $\mathrm{Ca}^{2+}$ and EF-hand binds $\mathrm{Ca}^{2+}$ selectively. On the contrary, when aspartate is present in the $12^{\text {th }}$ position, because of its smaller size, it can provide only one ligand, which can satisfy the hexa-coordination of $\mathrm{Mg}^{2+}$, but not the hepta coordination of $\mathrm{Ca}^{2+}$ and EF-hand binds $\mathrm{Mg}^{2+}$ preferentially. Besides coordinating the calcium ion as described above, the EF-hand loop possesses an extensive network of hydrogen bonds between chelating and non-chelating residues which stabilizes the loop (Gifford, Walsh et al. 2007).

\subsection{EF-hand structural reorganization.}

The EF-hand proteins that are $\mathrm{Ca}^{2+}$-sensors undergo structural rearrangement upon $\mathrm{Ca}^{2+}$ binding. The question is how does $\mathrm{Ca}^{2+}$ binding trigger structural reorganization in the EF-hand protein? As noted above, $\mathrm{Ca}^{2+}$ binding sites are located in the flexible loop that is located between entering and exiting helices of EF-hand. Residues 1, 3, 5, 7, and 9 of the loop provide five ligands for $\mathrm{Ca}^{2+}$ coordination. The rest two ligands are provided by the $12^{\text {th }}$ position of the loop (glutamic acid) which is located in the exiting helix. Glutamate residue at the $12^{\text {th }}$ position is far away to chelate the $\mathrm{Ca}^{2+}$. To complete the coordination sphere of $\mathrm{Ca}^{2+}$, the exiting helix is repositioned by $\sim 2 \AA$ and consequently, glutamic acid donates two ligands and fulfills the pentagonal bipyramidal geometry of $\mathrm{Ca}^{2+}$. Movement of the exiting helix leads to the conformational change in that EF-hand 
which is transmitted to other parts of the protein. $\mathrm{Ca}^{2+}$ induced structural reorganization can be exemplified by comparing the structure of ubiquitous $\mathrm{Ca}^{2+}$ binding protein calmodulin $(\mathrm{CaM})$, which contains four EF-hands, in the $\mathrm{Ca}^{2+}$ bound form and in $\mathrm{Ca}^{2+}$-free form (Figure 1.6). In the metal-free form of the $\mathrm{CaM}$, entering and exiting helices are antiparallelly oriented (interhelical angle $\sim 140^{\circ}$ ) and both helices experience extensive hydrophobic contacts (Figure 1.6, left); this conformation is known as closed conformation. Upon $\mathrm{Ca}^{2+}$ binding, helices become perpendicular and interhelical contact between the helices is lost; this conformation is called open conformation (Figure 1.6, right). This conformation change exposes the buried hydrophobic cavities, which enables $\mathrm{CaM}$ to interact with a wide range of targets. However, the extent by which EF-hand proteins undergo structural reorganization varies among proteins and within EF-hands of the same protein. For example, $\mathrm{Ca}^{2+}$ binding to metal-free recoverin, a protein associated

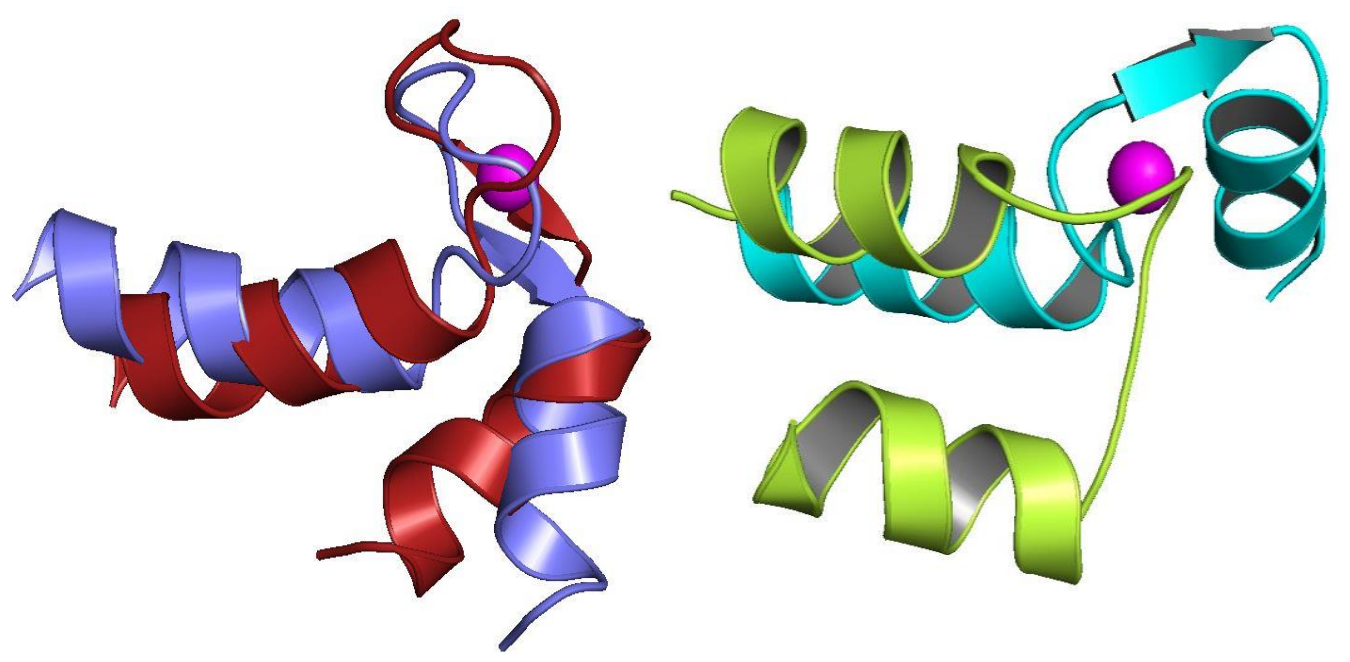

Figure 1.6. Structural reorganization of the EF-hands of CaM. Left) EF-1 in apo and $\mathrm{Ca}^{2+}$ bound forms are shown as firebrick color and slate colors, respectively. Right) EF-3 in apo and $\mathrm{Ca}^{2+}$-bound forms are shown as lime green and cyan, respectively. In both cases, $\mathrm{Ca}^{2+}$ is shown as magenta sphere. $\mathrm{Ca}^{2+}$ binding reorganizes both EF-hands from antiparallel to perpendicular orientation. PDB code of apo-CaM: $1 \mathrm{cfc}$; PDB code of $\mathrm{Ca}^{2+}-\mathrm{CaM}$ : $1 \mathrm{cll}$. 
with vision, does not lead to the pronounced structural reorganization; however, $\mathrm{Ca}^{2+}$ association leads to domain organization which exposes buried myristoylated group and enables recoverin to associate with the membrane (Figure 1.7). EF-hand proteins that act as calcium buffers, for example, parvalbumin and calbindin D-9k, undergo minimum structural reorganization upon $\mathrm{Ca}^{2+}$ association.
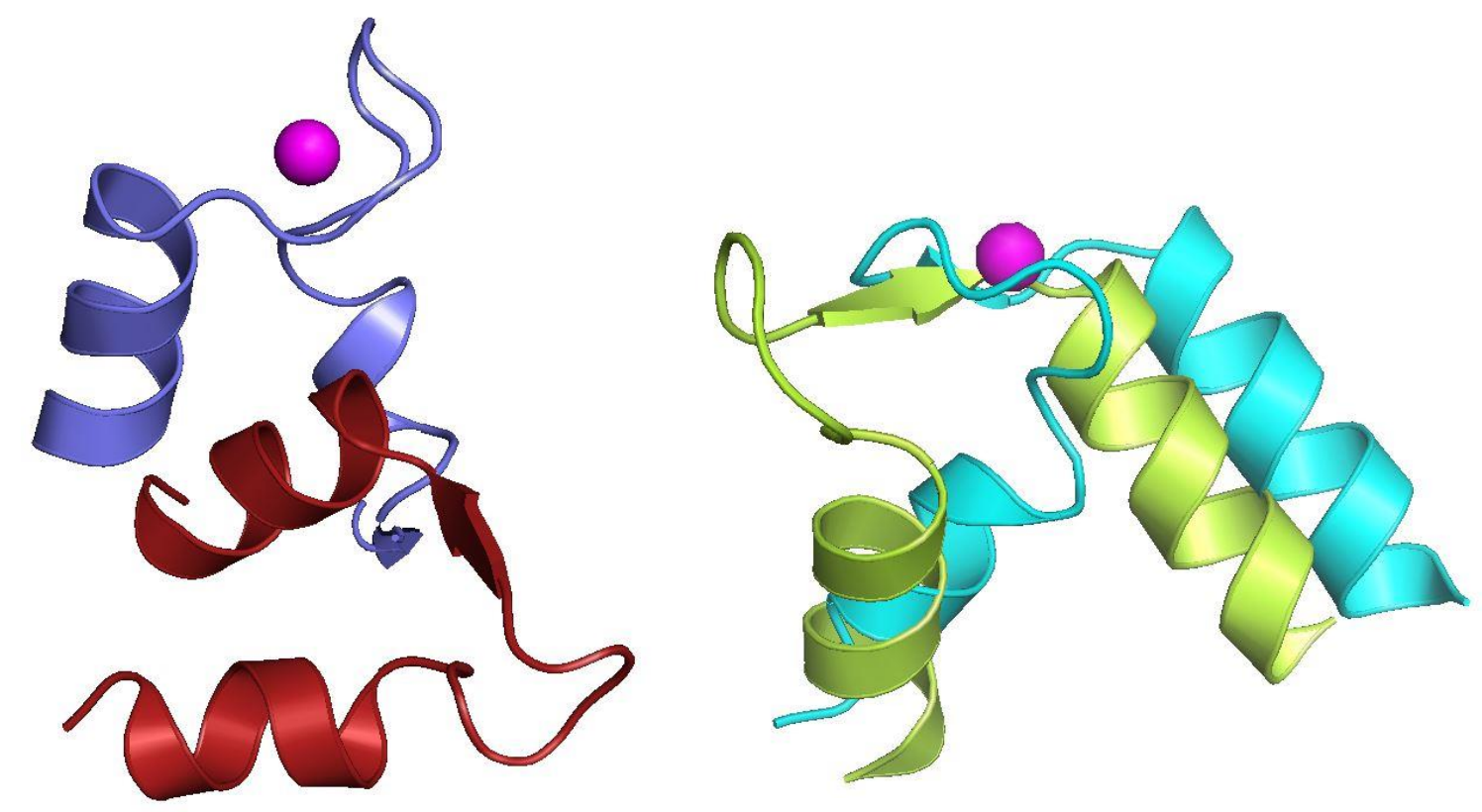

Figure 1.7. Structural reorganization of the EF-hands of recoverin. A) EF-1 in apo and $\mathrm{Ca}^{2+}$ bound forms are shown as firebrick and slate, respectively. B) EF-3 in apo and $\mathrm{Ca}^{2+}-$ bound forms are shown as lime green and cyan, respectively. In both cases, $\mathrm{Ca}^{2+}$ is shown as magenta sphere. $\mathrm{Ca}^{2+}$ binding does not lead to significant structural reorganization in the protein. PDB code of apo-recoverin: 1iku; PDB code of $\mathrm{Ca}^{2+}$-recoverin: 1jsa.

\subsection{Binding of non-physiological metals to EF-hand proteins.}

In addition to the binding of physiological ligands $\mathrm{Ca}^{2+}$ and $\mathrm{Mg}^{2+}$, EF-hand proteins have been shown to bind non-physiological ligands. A wide spectrum of studies has demonstrated that $\mathrm{Pb}^{2+}$ interacts with $\mathrm{EF}$-hand protein $\mathrm{CaM}$ and modulates its interaction with intracellular partners which might contribute to $\mathrm{Pb}^{2+}$-induced neurotoxicity 
(Gorkhali, Huang et al. 2016). For instance, $\mathrm{Pb}^{2+}$ displaces $\mathrm{Ca}^{2+}$ that is bound to EF-hands of $\mathrm{CaM}$ and $\mathrm{Pb}^{2+}: \mathrm{CaM}$ complex stimulates myosin light chain kinase and phosphodiesterase (Habermann, Crowell et al. 1983). Lead also has been shown to bind sites other than EF-hands, which could disrupt multiple downstream activities and induce neurotoxicity (Kern, Wisniewski et al. 2000, Goldstein, Ar 1983). Statistical analysis of $\mathrm{Pb}^{2+}$-bound proteins that were obtained from the protein data bank (PDB) have shown that negatively charged amino acid residues such as Asp and Glu provide ligands for $\mathrm{Pb}^{2+}$ coordination; the geometry of $\mathrm{Pb}^{2+}$-bound EF-hands is analogous to $\mathrm{Ca}^{2+}$-bound EF-hands as both $\mathrm{Pb}^{2+}$ and $\mathrm{Ca}^{2+}$ are coordinated by same binding ligands. Because of the preponderance of negatively charged amino acid residues in the EF-hand loop of EF-hand proteins, $\mathrm{Pb}^{2+}$ is a molecular target of a wide range of EF-hand proteins (Kirberger, Yang 2008).

Analogous to $\mathrm{Pb}^{2+}$ binding, $\mathrm{Cd}^{2+}$ also displaces $\mathrm{Ca}^{2+}$ from the EF-hand of CaM (Forsén, Thulin et al. 1980, Andersson, Forsen et al. 1983) which facilitates CaM interaction with myosin light chain kinase and phosphodiesterase (Suzuki, Chao et al. 1985, Chao, Bu et al. 1995, Mazzei, Girard et al. 1984). It has been proposed that competition between $\mathrm{Cd}^{2+}$ and $\mathrm{Ca}^{2+}$ for EF-hand of CaM is crucial for determining CaM toxicity at the molecular level (Suzuki, Chao et al. 1985, Mazzei, Girard et al. 1984). Few other studies have demonstrated that $\mathrm{Cd}^{2+}$ displaces $\mathrm{Ca}^{2+}$ from the EFhand of troponin C, a protein that regulates muscle contraction (Ellis, Strang et al. 1984, TELEMAN, DRAKENBERG et al. 1983). Cadmium shares similar properties with $\mathrm{Ca}^{2+}$ with ionic radii of $0.97 \AA$ and $0.99 \AA$ and charge/radius ratio $2.06 \mathrm{e} / \AA ̊$ and 2.02 e/Å, respectively (Choong, Liu et al. 2014). Computation studies have shown $\mathrm{Cd}^{2+}$ also has 
the tendency to be coordinated by negatively charged amino acid residues Asp and Glu (Friedman 2014, Jesu Jaya Sudan, Sudandiradoss 2012). So, both similar size and tendency of being coordinated by similar ligands support $\mathrm{Cd}^{2+}$ binding to EF-hands. In addition to divalent metal ions binding, trivalent lanthanide ions (e.g. $\mathrm{Tb}^{3+}, \mathrm{La}^{3+,}$ and $\mathrm{Lu}^{3+}$ have also shown to interact with EF-hand protein (Edington, Gonzalez et al. 2018, Gonzalez, Ramos et al. 2016). It has been demonstrated that lanthanide binding slightly distorts the geometry of the EF-hand loop as evidenced by the fact that lanthanide-bound CaM exhibits greater conformation flexibility and larger structural fluctuation than $\mathrm{Ca}^{2+}$-bound-CaM (Edington, Gonzalez et al. 2018). Using fluorescence and calorimetric techniques, it was shown that $\mathrm{Tb}^{3+}$ associates to neuronal calcium sensor (NCS) protein DREAM (Gonzalez, Ramos et al. 2016). Binding of monovalent ions to EF-hand protein is unlikely; however, one study has shown that monovalent ion $\mathrm{Na}^{+}$and $\mathrm{K}^{+}$can occupy EF-hand of parvalbumin which attenuates $\mathrm{Ca}^{2+}$ affinity for parvalbumin (Henzl, Larson et al. 2004b).

\subsection{Neuronal calcium sensor (NCS) proteins}

The neuronal calcium sensor (NCS) group is a subgroup of the EF-hand superfamily. Most members of this group are expressed in the brain and retina. The human genome comprises 14 NCS proteins, and the amino acid sequence of those members are highly conserved, especially in the EF-hand loop region (Ames, Lim 2012). These proteins regulate diverse neuronal processes, such as $\mathrm{Ca}^{2+}$ channel regulation, short-term synaptic plasticity, neuronal growth, neurotransmission regulation, light adaption (recoverin), antiapoptosis (hippocalcin), memory and learning (calsenilin, neuronal calcium sensor protein1), recycling and activation of guanylyl cyclase (visinin-like proteins 1 and 2, neurocalcin$\delta)$, potassium channels regulation (potassium channel interacting proteins (KChIP1-4), 
modulation of pain (KChIP3), and presenilin processing (KChIP 3 and 4) (Ames, Lim 2012, Burgoyne, Weiss 2001, Burgoyne 2007, Pongs, Lindemeier et al. 1993). Regulation of diverse neuronal processes could be explained by the fact that each NCS protein generates a unique $\mathrm{Ca}^{2+}$ signal that varies in duration, magnitude, and localization (Burgoyne, Weiss 2001, Burgoyne 2007). Additionally, regulation of the above processes depends on the localization of NCS proteins, the affinity of NCS proteins for $\mathrm{Ca}^{2+}$, and the interaction of NCS proteins with interacting partners (Burgoyne 2007). All NCS family members comprise four EF-hands and two distinct domains: EF-1 and EF-2 constitute the N-terminal domain; EF-3 and EF-4 constitute the C-terminal domain (Figure 1.8). In all NCS proteins, EF-hands always occur in pairs. Paired EF-hands in NCS proteins communicate through a short antiparallel $\beta$-sheet and because of this pairing protein display positive cooperativity for $\mathrm{Ca}^{2+}$ binding, that is, protein needs less $\mathrm{Ca}^{2+}$ to reach the

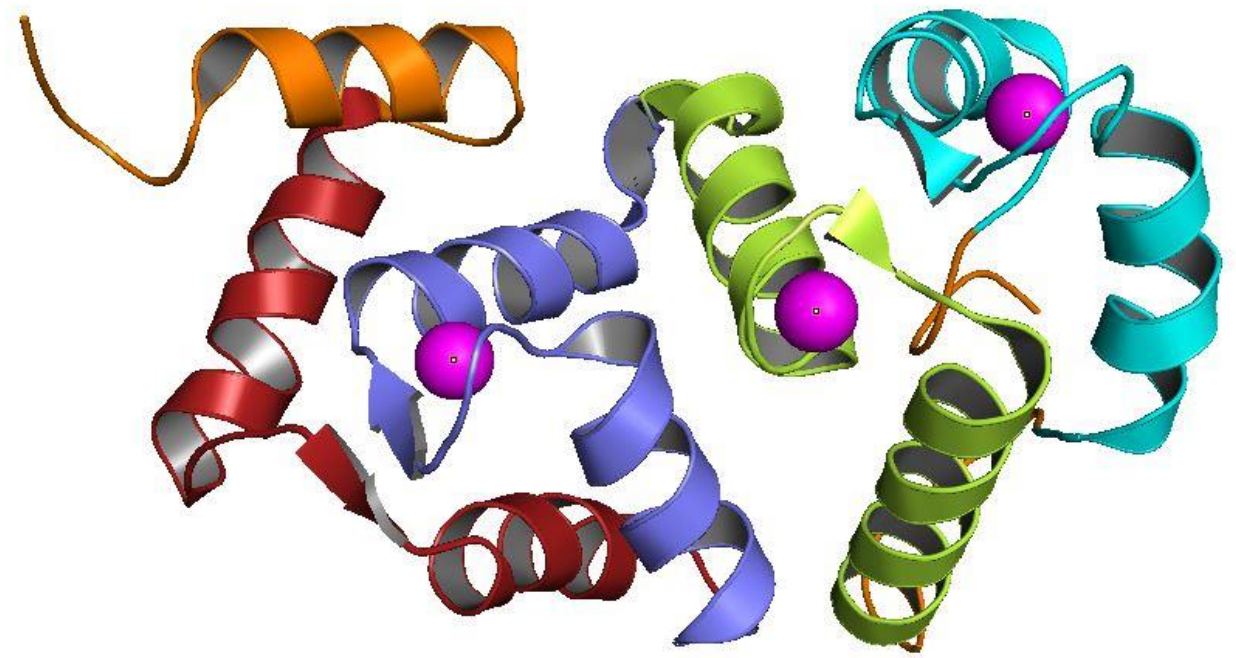

Figure 1.8. NMR structure of NCS-1 protein (PDB: 2lcp). EF-hands 1, 2, 3, and 4 are shown as firebrick, slate, lime green, and cyan, respectively; $\mathrm{Ca}^{2+}$ ions are shown as magenta spheres; and rest of the protein is shown as orange. EF-1 and EF-2 constitutes N terminus; EF-3 and EF-4 constitutes C-terminus. In each terminus, paired EF-hands communicate through a short antiparallel $\beta$-sheet. 
saturation (Ames, Lim 2012, Burgoyne, Weiss 2001). Four EF-hands of NSC proteins have distinct metal-binding properties: EF-1 is inactive in all members of the family as the presence of sterically hindered residue proline and cysteine in the EF-hand loop (conserved CPxG sequence, $\mathrm{x}$ any residue) distort $\mathrm{Ca}^{2+}$ binding geometry; $\mathrm{EF}-2$ and EF-3 binds $\mathrm{Ca}^{2+}$ with micromolar or submicromolar affinity in all members; and EF-4 is active in some members (Gifford, Walsh et al. 2007, Braunewell, Gundelfinger 1999, Ames, Lim 2012, Burgoyne, Weiss 2001, Haynes, Sherwood et al. 2007). Even though EF-1 of NCS proteins does not bind any $\mathrm{Ca}^{2+}$, some members contain a buried palmitoyl or myristoyl group near this EF-hand. Calcium binding to the C-terminus of some members has been shown to expose the prosthetic group which facilitates membrane association of those prosthetic groups (Figure 1.9) (Zozulya, Stryer 1992, Hughes, Brzovic et al. 1995, Ames, Tanaka et al. 1996). Each member of the NCS family has different $\mathrm{Ca}^{2+}$ affinity which depends on
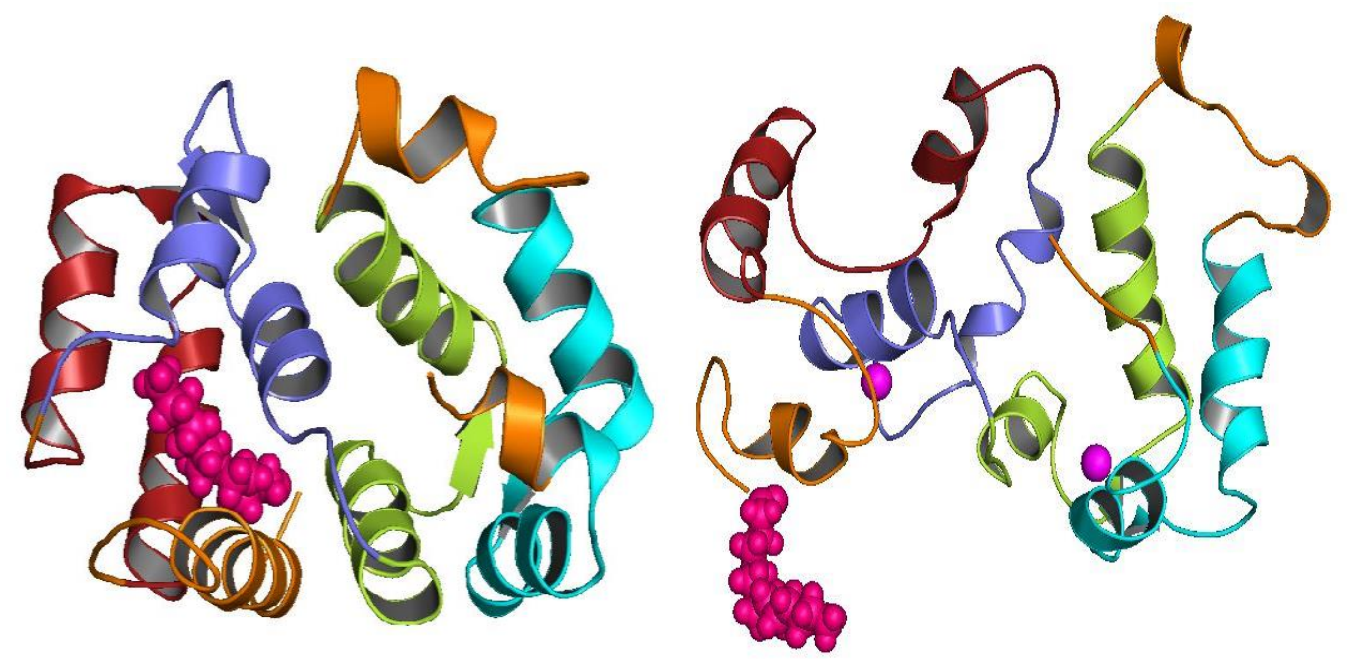

Figure 1.9. Left: NMR structure of $\mathrm{Ca}^{2+}$-free recoverin (PDB code: 1iku). Right: NMR structure of $\mathrm{Ca}^{2+}$-bound recoverin (PDB code: $1 \mathrm{jsa}$ ). In both cases, EF-hands $1,2,3$, and 4 are shown as firebrick, slate, lime green, and cyan, respectively. Rest of the protein is shown as cyan. $\mathrm{Ca}^{2+}$ ions are shown as magenta sphere. Myristoyl group is shown as pink. Myristoyl group is buried in $\mathrm{Ca}^{2+}$-free recoverin, but $\mathrm{Ca}^{2+}$ binding exposes the myristoyl group. 
amino acid sequence variation of each EF-hand (Burgoyne 2007). The NCS proteins have a flexible structure in the $\mathrm{Ca}^{2+}$-free form but become more ordered in $\mathrm{Ca}^{2+}$-bound form (Burgoyne, Weiss 2001). Burgoyne et al. classified NCS proteins into five classes (Burgoyne, Weiss 2001). Class A has only one member named frequenin. Frequenin is alternatively known as neuronal calcium sensor protein 1 (NCS-1). The NCS-1 contains a buried myristoyl group inside a hydrophobic moiety formed by the EF-3 and EF-4 in the C-terminus. In most NCS proteins, $\mathrm{Ca}^{2+}$ association exposes the buried myristoyl group, but in NCS-1, the extrusion of the myristoyl group is independent of $\mathrm{Ca}^{2+}$ association (O'Callaghan, Ivings et al. 2002). However, it has been shown that NCS-1 associates with lipid bilayer membrane strongly in the presence of $\mathrm{Ca}^{2+}$ (Handley, Lian et al. 2010). Class B comprises three members, named hippocalcin, neurocalcin- $\delta$ protein, visinin-like proteins (VILIP 1, 2, and 3). Biological properties of the members of class B are not well known as $\mathrm{Ca}^{2+}$ affinity and interactions of these proteins with binding partners vary (Braunewell, Szanto 2009). Class C contains only recoverin; it is first discovered NCS protein (Dizhoor, Ray et al. 1991). Recoverin is exclusively expressed in rod photoreceptors where it regulates vision processes through the inhibition of rhodopsin kinase in a $\mathrm{Ca}^{2+}$-dependent manner (Calvert, Klenchin et al. 1995, Makino, Dodd et al. 2004). In contrast to NCS-1 protein, where myristoyl group is sequestered in the hydrophobic cavity in the C-terminus, in recoverin, myristoyl group is buried inside the hydrophobic moiety formed by EF-1 and EF-2 in the N-terminus (Tanaka, Amest et al. 1995). It has been shown that binding of one $\mathrm{Ca}^{2+}$ at EF-3 is not sufficient for exposing the myristoyl group, but binding of two $\mathrm{Ca}^{2+}$ at EF-2 and EF-3 leads to an exposure of the myristoyl group from the hydrophobic cavity which facilitates the association of myristoyl 
group to the membrane as shown in Figure 1.9 (Ames, Lim 2012). Class D possesses guanylate cyclase-activating proteins (GCAPS) 1,2, and 3. These proteins are exclusively expressed in the photoreceptor cells and play important roles in vision processes by activating retinal guanylate cyclase at low $\mathrm{Ca}^{2+}$ concentration and inhibiting it at high $\mathrm{Ca}^{2+}$ concentration (Ames, Lim 2012, Dizhoor, Lowe et al. 1994). In contrast to other NCS proteins, $\mathrm{Ca}^{2+}$ association with GCPAs causes small conformational changes and therefore does not release the buried myristoyl group (Lim, Dizhoor et al. 2014). Additionally, EF2 and EF-3 of GCAPS are capable of binding both $\mathrm{Ca}^{2+}$ and $\mathrm{Mg}^{2+}$, but EF-3 binds $\mathrm{Ca}^{2+}$ selectively (Peshenko, Dizhoor 2006). The molecular mechanism of $\mathrm{Ca}^{2+}$ and $\mathrm{Mg}^{2+}$ binding to GCAP-1 has been resolved, but the mechanism of $\mathrm{Ca}^{2+}$ and $\mathrm{Mg}^{2+}$ binding to GCAPs 2 and 3 has not been deciphered yet. In metal-free form, GCAP-1 shows a flexible/disordered structure and does not interact and activate retinal guanylate cyclase (Peshenko, Dizhoor 2004, Lim, Peshenko et al. 2009). Magnesium $\left(\mathrm{Mg}^{2+}\right)$ binds at EF-2 of GCAP-1 and stabilizes the tertiary structure of GCAP-1, and the stabilization enables GCAP-1 to interact with retinal guanylate cyclase and subsequently activate it. Binding of three $\mathrm{Ca}^{2+}$ ions at EF-2, EF-3, and EF-4 of GCAP-1 leads to a distinct conformation that allows inhibition of retinal guanylate cyclase (Dizhoor, Lowe et al. 1994, Lim, Dizhoor et al. 2014, Peshenko, Dizhoor 2006). Class E contains four types of potassium channel interacting proteins (KChIPs) which are the latest member of the NCS family. These proteins are called potassium channel interacting proteins because they have been shown to interact with voltage-gated potassium channels (An, Bowlby et al. 2000). Each member of KChIPs has different isomers (five for KChIP-1, nine for KChIP-2, three for KChIP-3, and five for KChIP4) (Patel, Campbell et al. 2002). Experimental results from a northern blot study have unveiled that 
KChIP-1 is expressed in the brain, KChIP-2 is expressed in brain and heart, KChIP-3 is expressed in the brain and heart (An, Bowlby et al. 2000). The KChIP-4 was discovered from the database search of the proteins that have high sequence similarity with the first three members of the group (Holmqvist, Cao et al. 2002). All member of this group has high sequence homology in the Cterminus but differ each from other in the N-terminus (Patel, Campbell et al. 2002). Each member of the family has been shown to interact with the N-terminus of voltage-gated potassium channels ( $\mathrm{Kv}$ channels) and control the fast $\mathrm{I}_{\mathrm{TO}}$ and $\mathrm{I}_{\mathrm{SA}}$ currents in the heart and brain, respectively. It has been shown that KChIP-1, KChIP-2, and KChIP-3 associate with $\mathrm{K}_{\mathrm{V}} 4$ channels expressed in xenopus oocytes which causes rise in current density, slower inactivation kinetics, and acceleration of recovery from inactivation (An, Bowlby et al. 2000). All twenty-two isomers of the group interact with Kv4 channels in many different combinations which lead to a wide spectrum of

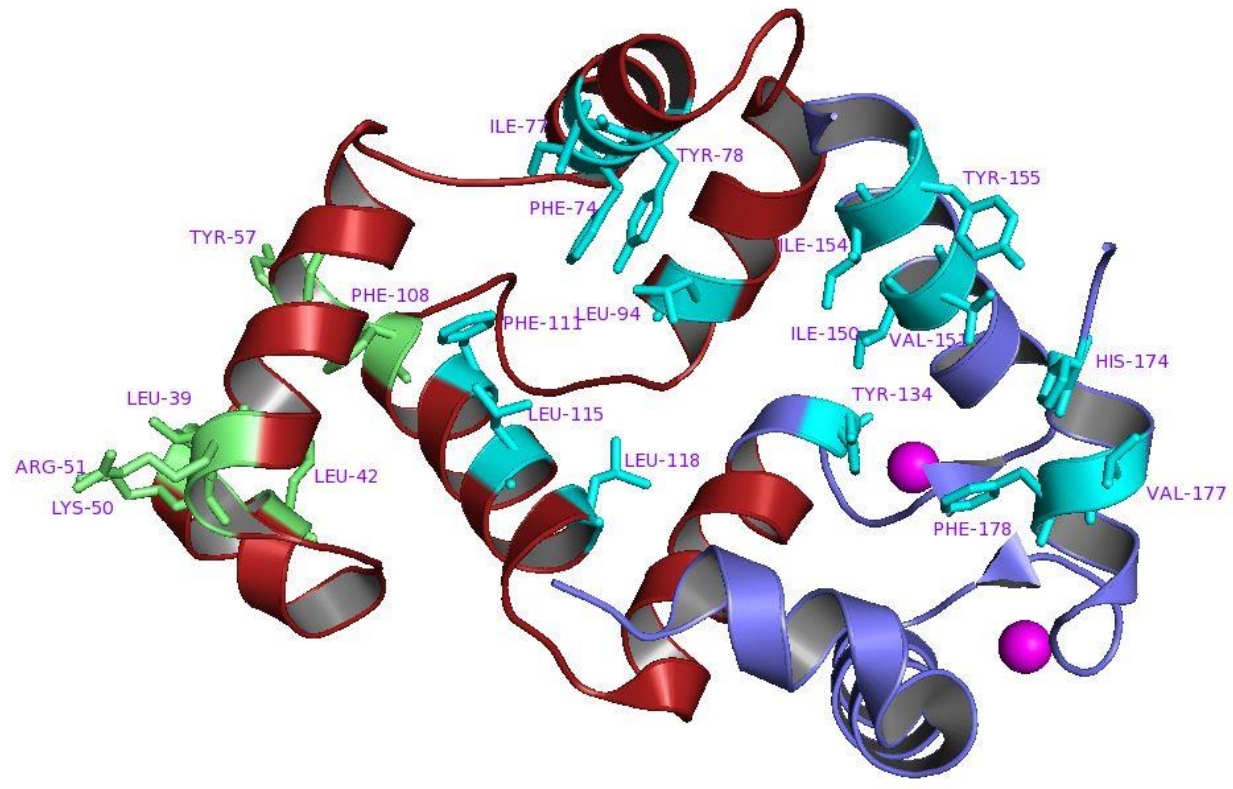

Figure 1.10. Representation of the amino acid residues of KChIP1 involved in interaction with $\mathrm{N}$-terminus of Kv4.3 channel (PDB: 2nz0). $\mathrm{N}$ terminus of the protein is shown in firebrick, C-terminus is shown in slate. Residues involved in first interface is shown in cyan sticks, residues involved in the second interface is shown in lime green sticks. 
potassium channel tuning (Bourdeau, Laplante et al. 2011, Liss, Franz et al. 2001, Kunjilwar, Qian et al. 2013). The X-ray crystallography study has demonstrated that KChIP-1 forms an octameric complex with $\mathrm{Kv} 4.3$, in which each KChIP-1 monomer interacts with two adjacent Kv4.3 Ntermini in a 4:4 manner (Pioletti, Findeisen et al. 2006, Wang, Yan et al. 2007b). Two contact interfaces are involved in KChIP-1: Kv4.3 interaction (Wang, Yan et al. 2007b). The most significant interaction occurs between hydrophobic cavities on the KChIP-1 surface and the hydrophobic $\alpha$-helix of the Kv4.3 channel. There are two hydrophobic cavities on the KChIP-1 surface. The N terminus residues of KChIP-1, Gly 59, Phe 60, Phe 74, Ile 77, Tyr 78, Leu94, Phe 111, Leu 115, and Leu 118, interact with Trp8 and Phe 11 of Kv4.3 channel (shown in cyan stick on firebrick background in Figure 1.10). The C-terminus residues, Tyr 134, Ile 150, Val 151, Ile 154, Tyr155, His174, and Phe178, interact with Trp19 of Kv4.3 channel (shown in cyan stick on slate background in Figure 1.10) (Wang, Yan et al. 2007b). The second contact interface is formed between residues 70-78 Kv4.3 channel and entering helix of EF-1 on KChIP-1 (Wang, Yan et al. 2007b). In the interface, Leu 39, Leu 42, Leu43, Tyr57, and Phe108 of KChIP-1 interacts with Phe73 of Kv4.3 channel through stacking interactions (shown in lime green stick on firebrick background in Figure 1.10). Furthermore, the interaction is stabilized by two salt bridge formations between Lys50 of KChIP-1 and Glu77 of Kv4.3 channel and between and Arg51 of KChIP-1 and Asp78 of Kv4.3 channel (Wang, Yan et al. 2007b).

Although all members of the KChIPs interact with voltage-gated potassium channels, only the structure of KChIP-1:Kv4 is available. On the basis of high sequence homology of KChIPs, especially the residues involved in the formation of the complex, other KChIPs could interact with Kv channel in an analogous manner (Liang, Wang et al. 2009). 


\subsection{Calsenilin/DREAM/KChIP3}

Interestingly, KChIP-3 has three different names. This protein was discovered by three independent groups and each group named it differently. On the basis of the fact that this protein interacts with presenilin and controls the activity of $\gamma$ - secretase complex, one research group named it calsenilin (Buxbaum, Choi et al. 1998). Later, another group named it as Downstream regulatory element antagonist modulator (DREAM) because the protein interacts with downstream regulatory element (DRE) and directly modulate transcription of downstream genes (Carrion, Link et al. 1999). Finally, the protein was renamed as KChIPs as it has high sequence similarity with KChIP-1 and KChIP-2 (An, Bowlby et al. 2000). I will use the name DREAM throughout. The DREAM is a $29 \mathrm{kDa}$ NCS protein that is expressed in the brain and heart and has been shown to control a wide spectrum of cellular processes (An, Bowlby et al. 2000, Buxbaum, Choi et al. 1998, Carrion, Link et al. 1999). The DREAM is the only $\mathrm{Ca}^{2+}$ binding protein that directly binds to DNA sequence (DRE) and represses transcription of genes (prodynorphin and c-fos genes) (Carrion, Link et al. 1999). In the $\mathrm{Ca}^{2+}$-free state, DREAM associates with DRE of human prodynorphin and c-fos genes and inhibit their transcription, but $\mathrm{Ca}^{2+}$ binding induces conformation change in the protein that prevents binding of DRE and subsequently reactivates the transcription (Carrion, Link et al. 1999, Cheng, Pitcher et al. 2002). Prodynorphin and c-fos genes have been shown to regulate pain modulation, cell homeostasis, and apoptosis (Dace et al. 2005, Osawa, Tong et al. 2001). The fact that DREAM represses transcription of DRE emphasize role of this protein regulating those processes (Costigan, Woolf 2002, Fontán-Lozano, Romero- 
Granados et al. 2009). In addition to direct regulation of gene expression, DREAM protein has been shown to indirectly regulates gene expression through interactions with CREB and $\alpha$-CREAM proteins. Interaction between DREAM and CREB enables regulation of genes that have promoters different than DRE (Ledo, Kremer et al. 2002).

In the cytoplasm, DREAM associates with carboxy-terminal fragment (CTF) of presenilin-1 (PS1) and presenilin-2 and stimulates the activity of $\gamma$ - secretase complex in a calcium-dependent manner and eventually overproduces $A \beta 42$ peptide, a peptide which has been linked to Alzheimer's disease (Buxbaum, Choi et al. 1998, Jo, Jang et al. 2005). The DREAM protein also interacts with the T1 domain of potassium channels and plays a prominent role in the regulation of fast $\mathrm{I}_{\mathrm{TO}}$ and ISA current in the heart and brain, respectively. The aforementioned interaction also facilitates the translocation of the channel to the membrane and alters the gating properties of the channel (An, Bowlby et al. 2000). Recently, Rashid et al. postulated that DREAM protein could be involved in the mechanism of nicotine treatment-prevented learning and memory impairment in REM sleep-deprived rats by altering its expression level in the hippocampus (Abd Rashid, Hapidin et al. 2017). It was also demonstrated that $\mathrm{Ca}^{2+}$-bound DREAM interacts with CaM and DREAM:CaM complex formation abolishes DREAM interaction DRE sites and promotes activation of calcineurin (Gonzalez, Arango et al. 2015, Ramachandran, Craig et al. 2012).

DREAM protein includes 256 amino acid residues. The structure of the first 77 residues of the DREAM has not been resolved yet because the poor solubility of the fulllength DREAM (residue 1-256) prevented structural determination of those residues by NMR study (Lusin, Vanarotti et al. 2008). After deletion of the first 64 residues, the 
solution structure of $\mathrm{Ca}^{2+}$ DREAM (residue 65-256) was resolved by Ames group (Lusin, Vanarotti et al. 2008). The DREAM protein remains fully functional after deletion of Nterminal 64 residues as evidenced by the fact that DREAM (residue 65-256) interacts with DNA, Kv.4 channel and the deletion does not impact $\mathrm{Ca}^{2+}$ induced oligomerization of the protein (Lusin, Vanarotti et al. 2008). The three-dimensional NMR structure of $\mathrm{Ca}^{2+}$ bound DREAM (residue 78-256) is shown in Figure 1.11. Like other NCS proteins, the DREAM protein contains two domains, and each domain possesses two EF-hands. The EF-1 (residue 90-119) and EF-2 (residue 128-157) coupled with a short N- terminus $\alpha$ helix constitute the N-terminal domain, whereas the EF3 (residue 163-192) and EF4 (residue 211-240) and C-terminal helix that is not part of the EF-hand constitute the Cterminal domain (Lusin, Vanarotti et al. 2008). The four EF-hands of DREAM protein differ in terms of metal binding property (Gifford, Walsh et al. 2007). The EF-hand 1 is

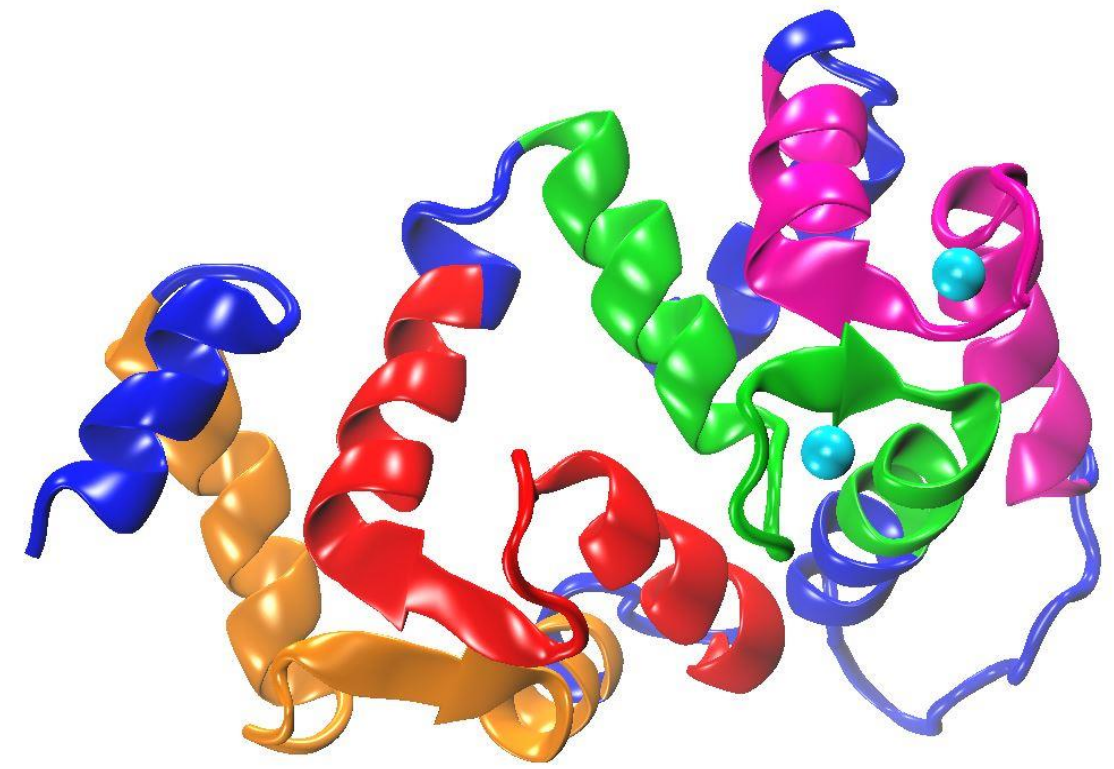

Figure1.11. Cartoon representation of $\mathrm{Ca}^{2+}$-bound DREAM(78-256) (PDB: 2jul). EF-hands 1, 2, 3, and 4 are shown in orange, red, green, and magenta, respectively. $\mathrm{Ca}^{2+}$ ions are shown in cyan spheres. Rest of the protein is shown in blue. For clariy, structure of first 77 residues were not resolved. 
not functional as the presence of sterically hindered amino acid residues in the EF-hand loop (CPXG sequence) perturb metal-binding geometry. Because of the presence of Asp in the $12^{\text {th }}$ position of the EF-hand loop instead of glutamate, the EF-2 binds $\mathrm{Mg}^{2+}$ preferentially. It has been shown that $\mathrm{Mg}^{2+}$ binding to EF-2 stabilizes the tertiary structure of DREAM and facilities DNA binding. The EF-hand 3 and EF-hand 4 bind $\mathrm{Ca}^{2+}$ preferentially with $\mathrm{K}_{\mathrm{d}}$ of 1-10 $\mu \mathrm{M}$ (Gifford, Walsh et al. 2007, Lusin, Vanarotti et al. 2008, Osawa, Dace et al. 2005, Osawa, Tong et al. 2001). Research from our lab demonstrated that EF-3 and EF-4 in DREAM bind non-physiological metal $\mathrm{Tb}^{3+}$ with higher affinity than $\mathrm{Ca}^{2+}$ (Gonzalez, Ramos et al. 2016).

The $\mathrm{N}$ - and C-terminal domain is connected through a central hydrophobic patch formed by the residues of $\alpha 4$ (Y130, F133, and A137), $\alpha 6$ (W169, L173), and $\alpha 7$ (I190, and M197)

(Figure 1.12). Furthermore, hydrophobic residues of EF-3 (L167, A170, M191, and L194),

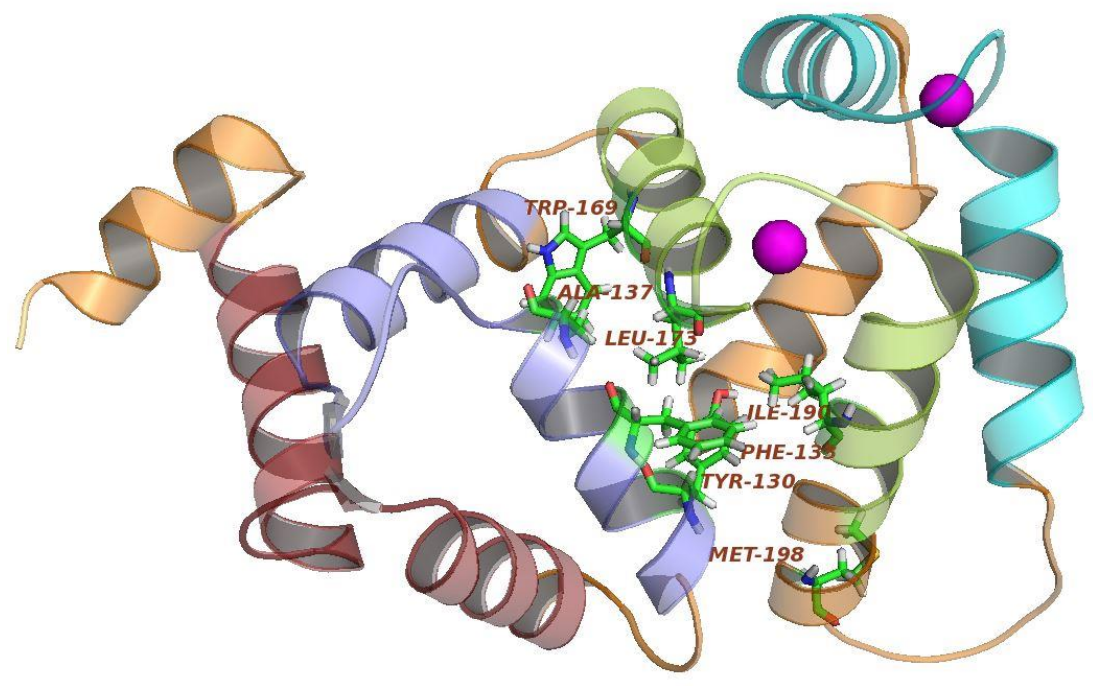

Figure 1.12. The central hydrophobic patch of the DREAM that communicate between $\mathrm{N}$ - and $\mathrm{C}$ - teriminal domain is shown in stick (PDB: 2jul). EF-hands 1, 2, 3, and 4 are shown in diffuse firebrick, slate, lime green, and cyan, respectively. Rest of the protein is shown in orange. $\mathrm{Ca}^{2+}$ ions are shown in magenta spheres. 
EF-4 (F218, and M222), and $\alpha 10$ (M246, M249, F252, and I256) constitute another hydrophobic patch at the C-terminal domain (Figure 1.13). The aforementioned hydrophobic patches play a prominent role in DREAM interaction with KV4.3 channels and hydrophobic molecules, including arachidonic acid and NS5806 (Gonzalez, Miksovska 2014, Gonzalez, Pham et al. 2014).

In the absence of any metal, DREAM exists as a tetramer, but $\mathrm{Ca}^{2+}$ binding triggers conformation changes in the protein that dissociates the tetramer into two dimers if the concentration of protein is above $150 \mu \mathrm{M}$. When the concentration of protein is above $250 \mu \mathrm{M}$ DREAM is tetramer in both metal-free forms and $\mathrm{Ca}^{2+}$ bound form (Osawa, Dace et al. 2005). However, to the best of my knowledge, the molecular mechanism of DREAM oligomerization has not been conclusively established yet. Lusin et al. proposed a model dimer structure in which N-terminus leucine residue region (Leu 155, Leu 158, Leu 159)

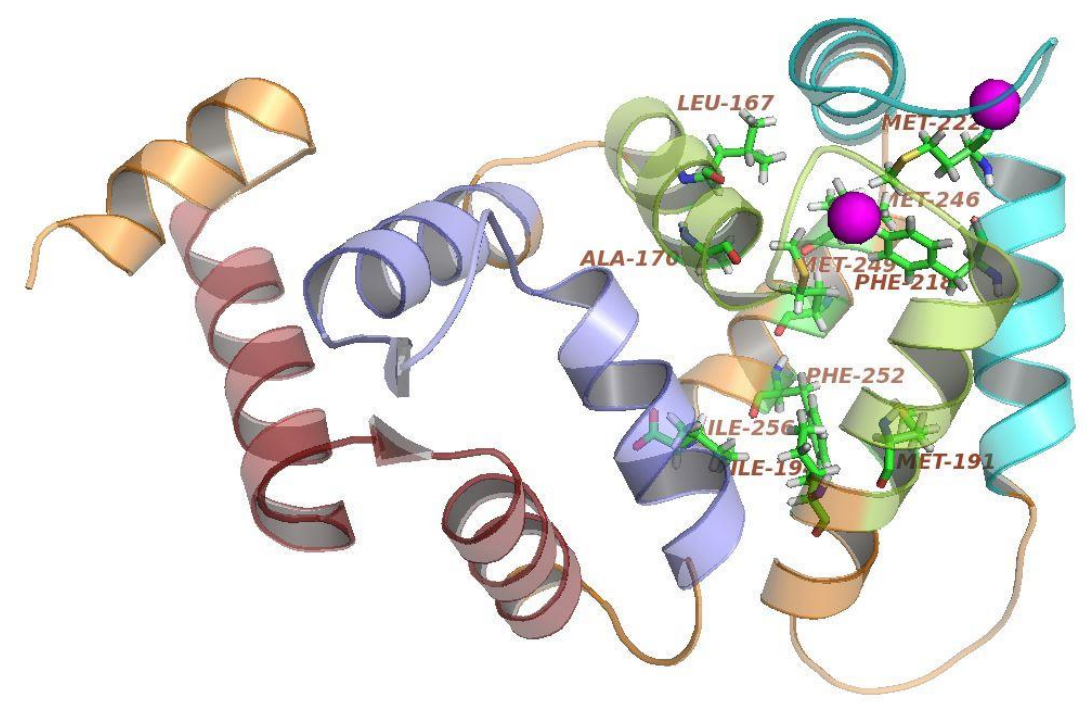

Figure 1.13. The C-terminal hydrophobic patch of DREAM is shown in stick (PDB: 2jul). EF-hands 1, 2, 3, and 4 are shown in diffuse firebrick, slate, lime green, and cyan, respectively. Rest of the protein is shown in orange. $\mathrm{Ca}^{2+}$ ions are shown in magenta spheres. 
of one monomer interacts with C-terminus Leu 251 of the second monomer in a head-totail fashion (Lusin, Vanarotti et al. 2008) (Figure 1.14: left panel). Docking study of our group has demonstrated that in addition to hydrophobic interaction, dimer could be stabilized through a salt bridge formation between positively charged amino acid residues (Arg 200 and Arg 207) of one monomer and negatively charged amino acid residue (Glu 103) of another monomer (Figure 1.14: right panel). The study of Lusin et al. also revealed a cluster of basic amino acid residues (Lys87, Lys90, Lys91, Arg98, Lys101, Arg160, and Lys166) at the N-terminus of DREAM (Figure 1.15). They proposed that these amino acid residues could be the potential DNA binding sites. The fact that many of that residue (Lys87, Lys90, Lys91, Lys101) are not conserved in the NCS proteins that do not bind DNA support their hypothesis. In the model dimer structure, the cluster of basic amino acid
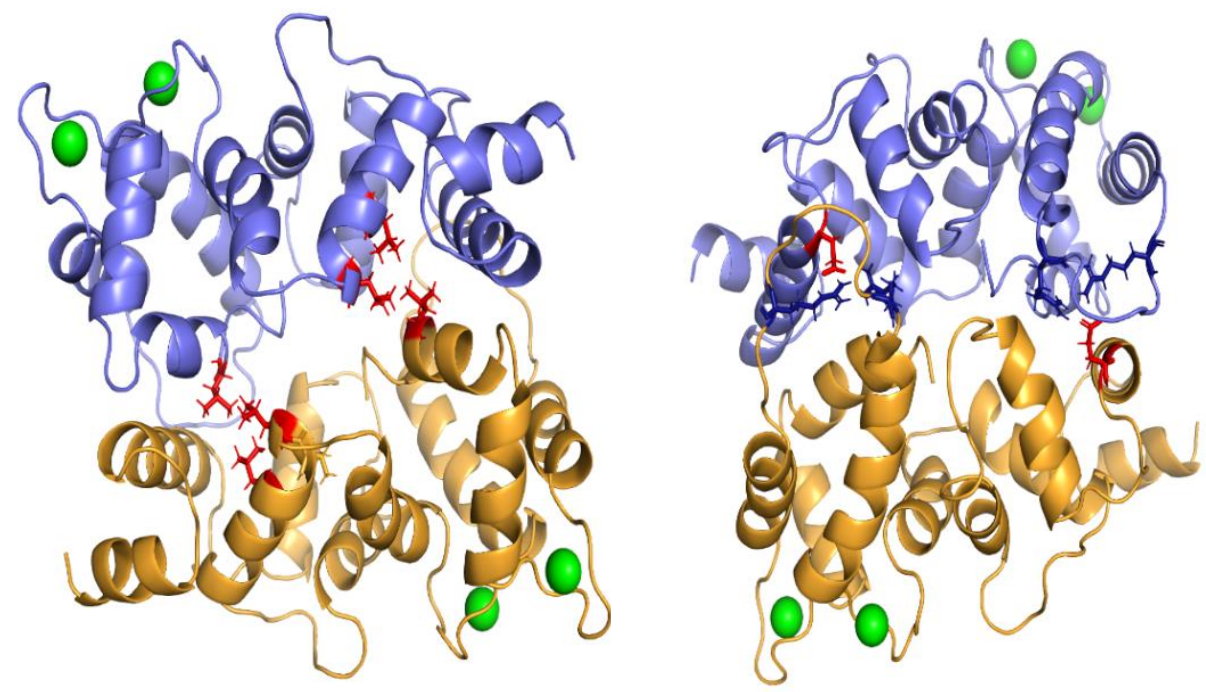

Figure 1.14. Model structure of DREAM dimer based on the NMR structure of DREM monomer (PDB entry 2JUL). Left panel shows hydrophobic interactions between Leu 155, 159 and 251 and right panel show a salt bridge between Arg 200, Arg 207 (in blue) and Glu 103 (in red). The two dimers were docked using Autodock and the initial structure of the dimer was refined in $50 \mathrm{~ns}$ molecular dynamic simulation. 
residues is sterically blocked, which might explain why $\mathrm{Ca}^{2+}$ binding abolishes DREAM interaction with DNA (Lusin, Vanarotti et al. 2008).

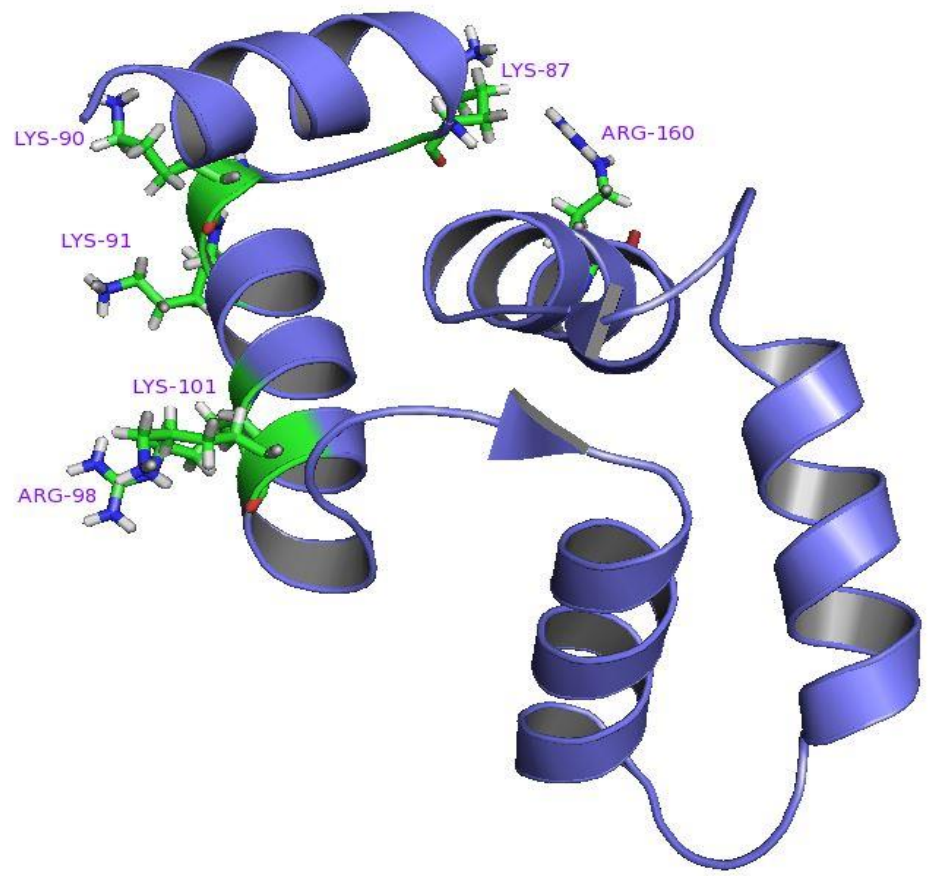

Figure 1.15. Cartoon representation of the N-terminus of DREAM (PDB: 2jul). Cluster of basic amino acid residues that are proposed to interact with DNA is shown as stick. 


\subsection{Objectives}

Objective 1:

Lead exposure has been associated with neurological and psychological disorders. Especially, children and seniors are vulnerable to $\mathrm{Pb}^{2+}$ exposure because $\mathrm{Pb}^{2+}$ exposure has been implicated with cognitive deficiencies and learning difficulties. It has been shown that $\mathrm{Pb}^{2+}$ displaces physiologically relevant ligands, such as $\mathrm{Ca}^{2+}, \mathrm{Mg}^{2+,}$ and $\mathrm{Zn}^{2+}$, from binding sites, which is believed to be one of the possible mechanisms of $\mathrm{Pb}^{2+}$ toxicity. Especially, $\mathrm{Ca}^{2+}$ binding proteins are molecular targets of $\mathrm{Pb}^{2+}$-induced toxicity. Lead has been shown to displace $\mathrm{Ca}^{2+}$ from the EF-hand of ubiquitous calcium-binding protein $\mathrm{CaM}$. Displacement of physiological ligand $\mathrm{Ca}^{2+}$ by $\mathrm{Pb}^{2+}$ was suggested to interfere with $\mathrm{CaM}$ interactions with downstream partners, and each interference represents a potential mechanism of $\mathrm{Pb}^{2+}$ induced toxicity. However, the exact mechanism through which $\mathrm{Pb}^{2+}$ induces toxicity has not been demystified yet. Statistical analysis of $\mathrm{Pb}^{2+}$ - and $\mathrm{Ca}^{2+}$ - bound protein has revealed that $\mathrm{Pb}^{2+}$ and $\mathrm{Ca}^{2+}$ are coordinated by analogous binding ligands (Asp, Glu) and $\mathrm{Pb}^{2+}$ binding does not distort the geometry of the EF-hand. $\mathrm{Pb}^{2+}$ exposure has been shown to impact the hippocampus region, the part of the brain that regulates memory and learning processes. On the basis of the fact that DREAM protein possesses a cluster of negatively charged amino and residues in the EF-hand regions, DREAM protein contains four EF-hands analogous to CaM, and DREAM protein is expressed in the hippocampus, we postulated that DREAM protein is a molecular target of $\mathrm{Pb}^{2+}$. Using spectroscopic techniques (fluorescence, $\mathrm{CD}$ ), we investigate whether $\mathrm{Pb}^{2+}$ addition induces the secondary and tertiary structural rearrangement of the DREAM and alters DREAM interactions with binding partners. We determine the affinity of $\mathrm{Pb}^{2+}$ for 
DREAM using isothermal titration calorimetry and fluorescence titration. Our results provided conclusive evidence of $\mathrm{Pb}^{2+}$ binding to DREAM. Because the DREAM protein shares high sequence homology with NCS proteins, other NCS proteins could bind $\mathrm{Pb}^{2+}$ with high affinity and explain the causes of $\mathrm{Pb}^{2+}$-induced toxicity.

Objective 2:

Cadmium exposure has been implicated with different types of cancers, neurodegenerative diseases. Children, pregnant women, and seniors are more susceptible to $\mathrm{Cd}^{2+}$ intoxication. It has been proposed that $\mathrm{Cd}^{2+}$ induces toxicity through interfering with $\mathrm{Ca}^{2+}$-and $\mathrm{Zn}^{2+}$-dependent processes, generating reactive oxygen species, inducing apoptosis, to name a few. But the exact mechanism of $\mathrm{Cd}^{2+}$-induced toxicity remains an active area of research. Previously, it has been shown that $\mathrm{Cd}^{2+}$ is capable of displacing $\mathrm{Ca}^{2+}$ from the EF-hand of $\mathrm{CaM}$ and troponin $\mathrm{C}$ and $\mathrm{Zn}^{2+}$ from protein kinase $\mathrm{C}$. Because $\mathrm{Cd}^{2+}$ binds to EF-hands of $\mathrm{CaM}$ and troponin $\mathrm{C}$ and DREAM is an EF-hand protein, we hypothesize that $\mathrm{Cd}^{2+}$ could bind to EF-hand of DREAM and displace the $\mathrm{Ca}^{2+}$. Utilizing spectroscopic techniques and analyzing data of four different DREAM mutants, we investigate whether $\mathrm{Cd}^{2+}$ is capable of displacing $\mathrm{Ca}^{2+}$ from the EF-hand, whether $\mathrm{Cd}^{2+}$ binding triggers structural rearrangement in the DREAM, and whether $\mathrm{Cd}^{2+}$ association impacts DREAM interaction with intracellular partners. We employ ITC and fluorescence titration for determining the affinity constant of $\mathrm{Cd}^{2+}$ for DREAM. Our results provide evidence of $\mathrm{Cd}^{2+}$ binding to DREAM for the first time, which could provide insight into $\mathrm{Cd}^{2+}$-induced toxicity. Our study could form the basis of NMR and X-ray crystallography on $\mathrm{Cd}^{2+}$-bound NCS proteins to provide atomic-level insight into the mechanism of $\mathrm{Cd}^{2+}$ interactions with NCS proteins. 


\section{Objective 3:}

Lithium has been used extensively for the treatment of psychological disorders for more than six decades. Increasing evidence from in vivo and in vitro studies in humans and rodents have implicated that $\mathrm{Li}^{+}$is a drug candidate for the treatment of neurogenerative diseases such as Parkinson's disease, Alzheimer's disease, and Huntington's disease. Despite having numerous clinical applications for treating devastating diseases, the exact mechanism through which $\mathrm{Li}^{+}$exerts its therapeutic action has not been resolved yet. Several mechanisms have been proposed. Among them, inhibitions of inositol monophosphatase (IMPase) is considered the most relevant mechanisms of action. Lithium treatment has been shown to inhibit IMPase by displacing native cofactor $\mathrm{Mg}^{2+}$. Lithium has been shown to associates with EF-hand protein neuronal calcium sensor-1 and alters its interaction with interacting partners. Lithium administration has been shown to alter the expression of voltage-gated potassium channel $4.2(\mathrm{Kv} 4.2)$ and potassium channel-interacting protein (KChIP-1). Since $\mathrm{Li}^{+}$binds to NCS-1 protein, which is homologous to DREAM, and impact the expression of KChIP-1, we investigate whether $\mathrm{Li}^{+}$directly binds to DREAM. Molecular dynamics simulation from our collaborator has identified potential $\mathrm{Li}^{+}$binding sites on DREAM. Using fluorescence spectroscopy and circular dichroism, we investigate whether $\mathrm{Li}^{+}$addition triggers structural rearrangement of DREAM, whether $\mathrm{Li}^{+}$addition impact DREAM interactions with binding partners. Our results provided the binding of monovalent ion to DREAM protein for the first time. Results from the study suggest that DREAM and probably other NCS proteins are the molecular targets of $\mathrm{Li}^{+}$. 


\section{Objective 4:}

The aMD data indicate the presence of a salt bridge between Lys87 and Asp 165 of DREAM that couples the loop between helix-1 and helix-2 in the N-terminal domain with the EF-3 in the C-terminal domain. We postulate that the salt bridge could be involved in the propagation of calcium-triggered structural changes between the C- terminal and $\mathrm{N}$ terminal domain. To determine the role of Lys 87 to the interdomain communication Lys87 was mutated to Ala and the consequence of the mutation on DREAM secondary and tertiary structures and DREAM interactions with interacting partners were probed.

Objective 5:

The $\mathrm{Ca}^{2+}$ binding protein DREAM binds $\mathrm{Ca}^{2+}$ at EF-3 and EF-4. We and other researchers demonstrated that $\mathrm{Ca}^{2+}$ binding causes secondary and tertiary structural rearrangements of the protein and consequently alters DREAM interaction with intracellular partners. But exact mechanism of $\mathrm{Ca}^{2+}$-triggered structural rearrangements of DREAM and modulation of interactions with binding partners remains unknown. In the present study, we mutated glutamate residue at $12^{\text {th }}$ position of EF-3 (DREAM(E186Q)) and EF-4 (DREAM(E234Q)) to glutamine for making these $\mathrm{EF}$-hands $\mathrm{Ca}^{2+}$ insensitive and investigated whether mutations impact $\mathrm{Ca}^{2+}$-induced secondary and tertiary structural rearrangements of the protein and whether mutations impact $\mathrm{Ca}^{2+}$-induced modulation of DREAM interaction with T-1 domain of Kv channel. 


\section{MATERIALS AND METHODS}

\subsection{Methods used in chapter 3}

\subsubsection{General}

The $\mathrm{Pb}\left(\mathrm{CH}_{3} \mathrm{COO}\right)_{2 .} 3 \mathrm{H}_{2} \mathrm{O}$ was purchased from Fisher Scientific. The $\mathrm{Pb}^{2+}$ stock was prepared in $20 \mathrm{mM}$ Tris $\mathrm{pH} 7.4$ (prepared in decalcified ultrapure $18 \mathrm{M} \Omega$ water). The hydrophobic fluorescent probe 1,8-ANS was purchased from Invitrogen and was used without further purification. Peptides with sequences corresponding to the sequence of the site-1 (FITC-AAGVAAWLPFARAAAIGWMPV) and site-2 ((FITCLLGSTEKEFFFNEDTKEYFFD) of human $\mathrm{K}_{\mathrm{v}} 4.3$ were purchased from Think Peptides and received at higher than $98 \%$ purity. The synthetic peptide derived for helix -9 of presenilin-1 "PS1HL9” (residues 445FYFATDYLVQPFMDQLAFHQFYI467) of human PS1-CTF with FITC covalently attached to the amino-terminus was purchased from ProImmune (Sarasota, FL, USA). All other chemicals were obtained from Sigma-Aldrich and Fisher Scientific unless stated otherwise.

\subsubsection{Isolation and purification of $\operatorname{DREAM}(\Delta 64)$ and $\operatorname{DREAM}(\Delta 160)$}

Recombinant mouse $\operatorname{DREAM}(\Delta 64)$ (residues 65-256) and $\operatorname{DREAM}(\Delta 160)$ (residues 161-256) with the C-terminus His-tag were expressed in E. coli BL21 (DE3) cells and purified according to previously published procedures with minor modifications (Osawa, Tong et al. 2001). Briefly, plasmids encoding for the his-tagged mouse $\operatorname{DREAM}(\Delta 64)$ and his-tagged DREAM $(\Delta 160)$ were purchased from GeneCopoeia and transformed in E. coli BL21(DE3) competent cells and grown in TB medium at $37^{\circ} \mathrm{C}$ in the presence of $100 \mu \mathrm{g} / \mathrm{mL}$ ampicillin until reaching an optical density of 0.8 at $600 \mathrm{~nm}$. Protein expression was induced by adding $0.5 \mathrm{mM}$ IPTG. The medium was placed back in 
the shaker at $250 \mathrm{rpm}$ for an additional 12 hours at room temperature followed by the harvesting of the cells by centrifugation for 20 minutes at $5000 \mathrm{rpm}$. The cell pellet was resuspended in lysis buffer (100 $\mathrm{mM}$ Tris buffer $\mathrm{pH} 8.0,0.5 \mathrm{M} \mathrm{NaCl}, 1 \mathrm{mM} \mathrm{CaCl}_{2}, 5 \mathrm{mM}$ $\mathrm{MgCl}_{2}, 5 \mathrm{mM}$ imidazole, $1.0 \%$ Triton-X100, $0.5 \%$ Tween 20, and 10\% glycerol), supplemented with $20 \mu \mathrm{g} / \mathrm{mL}$ DNAse, and $200 \mu \mathrm{g} / \mathrm{mL}$ lysozyme and sonicated in an ice bath for 30 cycles at $30 \%$ duty cycle. The cell lysate was centrifuged at $5000 \mathrm{rpm}$ for 4 hours, and the supernatant was loaded to a Ni-NTA column equilibrated with buffer A (20 $\mathrm{mM}$ Tris $\mathrm{pH} 7.4,300 \mathrm{mM} \mathrm{NaCl}, 0.5 \mathrm{mM} \mathrm{CaCl}_{2}$, and $5 \mathrm{mM}$ imidazole). The Ni-NTA column loaded with DREAM was washed extensively with buffer $A$ and an increasing concentration of imidazole $(5-40 \mathrm{mM})$. Protein was eluted in buffer A and $250 \mathrm{mM}$ imidazole. Selected fractions were loaded onto a DEAE-Sepharose anion exchange column previously equilibrated with $20 \mathrm{mM}$ Tris $\mathrm{pH}$ 7.4, washed with $20 \mathrm{mM}$ Tris $\mathrm{pH} 7.4$ and 50 $\mathrm{mM} \mathrm{NaCl}$, and then eluted with $20 \mathrm{mM}$ Tris $\mathrm{pH} 7.4$ and $200 \mathrm{mM} \mathrm{NaCl}$. After anion exchange, the protein was dialyzed twice against two liters of $20 \mathrm{mM}$ Tris $\mathrm{pH} 7.4$ for 12 hours. The purified protein was stored in $20 \mathrm{mM}$ Tris $\mathrm{pH}$ 7.4. The purity of all protein constructs was assessed using SDS-PAGE. The proper secondary structure and tertiary structure of all protein constructs were confirmed by using the known far-UV CD transition of DREAM protein as well as changes in tryptophan fluorescence upon calcium binding (Gonzalez, Arango et al. 2015, Osawa, Dace et al. 2005, Osawa, Tong et al. 2001).

\subsubsection{Sample preparation}

Protein samples were prepared from stock in $20 \mathrm{mM}$ Tris $\mathrm{pH}$ 7.4. Protein concentrations were measured by UV-VIS spectrophotometry using $\varepsilon_{280 \mathrm{~nm}}=19,000 \mathrm{M}^{-1} \mathrm{~cm}^{-}$

${ }^{1}$ for DREAM $(\Delta 64)$ and $\varepsilon_{280 \mathrm{~nm}}=10,544 \mathrm{M}^{-1} \mathrm{~cm}^{-1}$ for DREAM $(\Delta 160)$. The concentrations 
of 1,8-ANS and $\mathrm{K}_{\mathrm{V}} 4.3(2-22)$ "site-1", $\mathrm{K}_{\mathrm{V}} 4.3(70-90)$ "site-2" and helix-9 of presenilin-1 "PS1HL9" were measured by using $\varepsilon_{350 \mathrm{~nm}}=4999 \mathrm{M}^{-1} \mathrm{~cm}^{-1}$ and $\varepsilon_{493 \mathrm{~nm}}=75000 \mathrm{M}^{-1} \mathrm{~cm}^{-1}$, respectively. $\mathrm{Ca}^{2+}, \mathrm{Pb}^{2+}$, EDTA, 1,8-ANS, Kv4.3(2-22) “site-2”, Kv4.3(70-90) “site-2”, and helix-9 of presenilin-1 "PS1HL9" were added to the sample to obtain the desired concentration.

\subsubsection{Steady-state emission spectra}

Steady-state emission spectra were measured at room temperature $\left(20^{\circ} \mathrm{C}\right)$ in a 0.2 $\mathrm{x} 1-\mathrm{cm}$ path length quartz cuvette with excitation along the $0.2-\mathrm{cm}$ path using a custom Chronos ${ }^{\mathrm{FD}}$ spectrofluorometer (ISS, Champaign IL). For the Trp emission measurement, samples were excited at $295 \mathrm{~nm}$, whereas for 1,8-ANS emission measurement, samples were excited at $350 \mathrm{~nm}$. To determine the equilibrium dissociation constant for $\mathrm{Pb}^{2+}$ binding to apoDREAM, small aliquots of $\mathrm{Pb}^{2+}$ stock solution $\left(500 \mu \mathrm{M} \mathrm{Pb}\left(\mathrm{CH}_{3} \mathrm{COO}\right)_{2}\right.$ dissolved in $20 \mathrm{mM}$ Tris $\mathrm{pH} 7.4)$ was added to $20 \mu \mathrm{M} \operatorname{DREAM}(\Delta 64)$ in the presence of $100 \mu \mathrm{M} \mathrm{Ca}^{2+}, 20 \mathrm{mM}$ Tris buffer $\mathrm{pH}$ 7.4. The fraction of $\mathrm{Pb}^{2+}$-bound protein $\left(\mathrm{f}_{\mathrm{b}}\right)$ was determined from the decrease in Trp emission intensity at $329 \mathrm{~nm}$ using Equation 1.

$$
\mathrm{f}_{\mathrm{b}}=\frac{\left(\mathrm{F}_{\mathrm{i}}-\mathrm{F}_{0}\right)}{\left(\mathrm{F}_{\max }-\mathrm{F}_{0}\right)}
$$

Where $\mathrm{F}_{\mathrm{i}}$ is the fluorescence intensity in different concentrations of $\mathrm{Pb}^{2+} ; \mathrm{F}_{\mathrm{o}}$ is the fluorescence intensity in the absence of $\mathrm{Pb}^{2+}$, but in the presence of $100 \mu \mathrm{M} \mathrm{Ca}^{2+}$; and $\mathrm{F}_{\max }$ is the saturation fluorescence intensity due to $\mathrm{Pb}^{2+}$ addition. The equilibrium dissociation constants $\left(\mathrm{K}_{\mathrm{d}}\right)$ were determined using single site binding model (Equation 2).

$$
\mathrm{f}_{\mathrm{b}}=\frac{\left(\mathrm{K}_{\mathrm{d}}+\left[\mathrm{nP}_{\mathrm{t}}\right]+\left[\mathrm{L}_{\mathrm{t}}\right]\right)-\sqrt{\left(\mathrm{K}_{\mathrm{d}}+\left[\mathrm{nP}_{\mathrm{t}}\right]+\left[\mathrm{L}_{\mathrm{t}}\right]\right)^{2}-4\left[\mathrm{nP}_{\mathrm{t}}\right]\left[\mathrm{L}_{\mathrm{t}}\right]}}{\left[\mathrm{nP}_{t}\right]} \ldots
$$


where $\mathrm{P}_{\mathrm{t}}$ is total protein concentration, $\mathrm{L}_{\mathrm{t}}$ is the total ligand concentration, and $\mathrm{n}$ is the number of binding sites. For direct titration data such as 1,8 -ANS binding to $\mathrm{Pb}^{2+}-$ $\operatorname{DREAM}(\Delta 64)$ and peptide binding to $\operatorname{Pb}^{2+}-\operatorname{DREAM}(\Delta 64), \mathrm{K}_{\mathrm{d}}$ in Equation 2 corresponds to the equilibrium dissociation constant. In the case of displacement studies, $\mathrm{K}_{\mathrm{d}}$ represents $\mathrm{K}_{\text {app }}$ for $\mathrm{Pb}^{2+}$ binding to $\mathrm{Ca}^{2+}$-DREAM $\left(\Delta 64\right.$. The reported $\mathrm{K}_{\text {app }}$ or $\mathrm{K}_{\mathrm{d}}$ value represents the mean and standard deviation from three independent measurements. Based on the apparent dissociation constant for $\mathrm{Pb}^{2+}$ binding to $\mathrm{Ca}^{2+}$ bound DREAM, the dissociation constant $\left(\mathrm{K}_{\mathrm{d}}\right)$ for $\mathrm{Pb}^{2+}$ binding to $\mathrm{Ca}^{2+}$ free DREAM was calculated using Equation 3 (Kirberger, Wong et al. 2013).

$$
\mathrm{K}_{\mathrm{d}}=\frac{\mathrm{K}_{\mathrm{app}} \mathrm{KdCa}^{2+}}{\left(\mathrm{KdCa}^{2+}\left[\mathrm{Ca}^{2+}\right] \mathrm{T}\right)}
$$

where $\mathrm{K}_{\mathrm{d}}$ is the dissociation constant of $\mathrm{Pb}^{2+}$ binding to apoDREAM, $\mathrm{K}_{\mathrm{app}}$ is the apparent dissociation constant of $\mathrm{Pb}^{2+}$ binding to $\mathrm{Ca}^{2+}$ DREAM, $\mathrm{K}_{\mathrm{d}^{2} a^{2+}}$ is the equilibrium dissociation constant for $\mathrm{Ca}^{2+}$, which was reported previously to be $1 \mu \mathrm{M}$ (Osawa, Dace et al. 2005); $\left[\mathrm{Ca}^{2+}\right]_{\mathrm{T}}$ is the total concentration of $\mathrm{Ca}^{2+}$, which is $100 \mu \mathrm{M}$.

To determine the affinity for 1,8-ANS binding to $\mathrm{Pb}^{2+}$-bound DREAM( $\left.\Delta 64\right), 10 \mu \mathrm{M} 1,8$ ANS was prepared in $20 \mathrm{mM}$ Tris in the presence of $40 \mu \mathrm{M} \mathrm{Pb}^{2+}$. Stocks of DREAM( $\left.\Delta 64\right)$ were prepared with $40 \mu \mathrm{M} \mathrm{Pb}^{2+}$ and $10 \mu \mathrm{M}$ 1,8-ANS. Aliquots of DREAM( $\left.\Delta 64\right)$ stock were added to $10 \mu \mathrm{M}$ 1,8-ANS and the emission spectra were recorded. The increase in emission intensity at the emission maximum of $466 \mathrm{~nm}$ was used to calculate the fraction of 1,8-ANS bound to protein by Equation 1, and data were fitted to Equation 2 to determine the dissociation constant for 1,8-ANS. 


\subsubsection{Circular dichroism}

Circular dichroism measurements were carried out using a J-810 Jasco CD spectrometer at room temperature $\left(20^{\circ} \mathrm{C}\right)$. For far-UV CD, $20 \mu \mathrm{M}$ DREAM $(\Delta 64)$ in $20 \mathrm{mM}$ Tris pH 7.4 and $1 \mathrm{mM}$ EDTA, $1 \mathrm{mM} \mathrm{Ca}^{2+}$, or $40 \mu \mathrm{M} \mathrm{Pb}^{2+}$ was placed in a quartz cuvette $(1$ $\mathrm{mm} \times 10 \mathrm{~mm}$ ) and data were collected through $1 \mathrm{~mm}$ path from 200 to $250 \mathrm{~nm}$. Amount of secondary structure content was obtained using secondary structure prediction tool K2D3 program. For near UV CD, $40 \mu \mathrm{M}$ DREAM $(\Delta 64)$ in $5 \mathrm{mM}$ phosphate $\mathrm{pH} 7.4$ in the presence of $1 \mathrm{mM}$ EDTA, $1 \mathrm{mM} \mathrm{Ca}^{2+}$, or $40 \mu \mathrm{M} \mathrm{Pb}^{2+}$ was placed in a quartz cuvette $(1 \mathrm{~mm} \times 10$ $\mathrm{mm}$ ) and data were recorded through $1 \mathrm{~cm}$ path from $250 \mathrm{~nm}$ to $330 \mathrm{~nm}$. The baseline was subtracted from each spectrum.

\subsubsection{Fluorescence lifetime measurements}

Frequency-domain fluorescence lifetime measurements were performed at room temperature $\left(20^{\circ} \mathrm{C}\right)$ using a $\mathrm{Chronos}^{\mathrm{FD}}$ spectrofluorometer (ISS, Champaign IL). 1,8ANS:DREAM complex was excited with the output $300 \mathrm{~nm}$ laser diode through $305 \mathrm{~nm}$ bandpass filter and emission were collected through $400 \mathrm{~nm}$ long pass and $600 \mathrm{~nm}$ shortpass filter. For Trp lifetime measurements, samples were excited using an output of 280 $\mathrm{nm}$ laser diode and emission was collected through $320 \mathrm{~nm}$ long-pass filter. 1,4-bis(5phenyloxazol-2-yl)benzene (POPOP) in ethanol was used as a reference in lifetime measurements $(\tau=1.35 \mathrm{~ns})$. For 1,8-ANS:DREAM complexes lifetime measurements, modulation-phase data were analyzed using three discrete exponential decay component, whereas for Trp lifetime measurements, data were fitted by using a sum of a Gaussian distribution model and a single exponential decay model in Global software. For Trp 
lifetime data, discrete lifetime $\tau_{2}$ was used as a linked variable in the Global Analysis. The $\chi^{2}$ parameter and residuals were used as a criterion for the goodness of the fit.

\subsubsection{ITC measurements}

Isothermal calorimetry titrations were used to determine the thermodynamics of displacement of $\mathrm{Ca}^{2+}$ from DREAM $(\Delta 64)$ by $\mathrm{Pb}^{2+}$. Titrations were carried out using a VPITC isothermal calorimeter (Microcal Inc. Northampton, MA). Protein solutions were prepared in $\left(5 \mathrm{mM}\right.$ Tris $\left.\mathrm{pH} 7.4,100 \mu \mathrm{M} \mathrm{CaCl}_{2}\right)$ buffer and dialyzed overnight three times in the same buffer. $\mathrm{Pb}^{2+}$ stock solutions were prepared in an ITC dialysate buffer. The reaction cell was loaded with $\sim 20 \mu \mathrm{M}$ protein solution in $5 \mathrm{mM}$ Tris $\mathrm{pH} 7.4,100 \mu \mathrm{M} \mathrm{CaCl}_{2}$ buffer, and the protein concentration was determined spectrophotometrically prior to the ITC experiment. The concentration of $\mathrm{Pb}^{2+}$ in the syringe was $400 \mu \mathrm{M} ; 30$ aliquots $(6 \mu \mathrm{L}$ each) of $400 \mu \mathrm{M} \mathrm{Pb}^{2+}$ stock were titrated into $20 \mu \mathrm{M}$ DREAM( $\left.\Delta 64\right)$. The ITC experiments were conducted three times at $25^{\circ} \mathrm{C}$. Thermodynamic parameters were obtained by fitting the isotherms with the sequential model ( 2 sets of sites) using the Microcal ITC analysis plug-in Origin 7.0. Overall apparent dissociation constant was obtained from individual association constants using Equation 4 (Aravind, Chandra et al. 2008).

$$
\mathrm{K}_{\mathrm{app}}=\frac{1}{\sqrt{\mathrm{K}_{1} \mathrm{~K}_{2}}}
$$

Where $\mathrm{K}_{\text {app }}$ represents the overall apparent dissociation constant for $\mathrm{Pb}^{2+}$ binding to $\mathrm{Ca}^{2+} \operatorname{DREAM}(\Delta 64)$, whereas $\mathrm{K}_{1}$ and $\mathrm{K}_{2}$ represent apparent association constants of $\mathrm{Pb}^{2+}$ binding to $\mathrm{Ca}^{2+} \operatorname{DREAM}(\Delta 64)$. 


\subsubsection{Steady-state polarization measurements}

The steady-state polarization measurements were carried out in a Chronos ${ }^{\mathrm{FD}}$ spectrofluorometer (ISS, Champaign IL). To investigate the interaction between $\operatorname{DREAM}(\Delta 64)$ and FITC-labeled PS1HL9, FITC-labeled site-1, and FITC-labeled site-2, the samples were excited at $493 \mathrm{~nm}$, and the emitted light was collected through a $500 \mathrm{~nm}$ long-pass filter. Binding of site-1, site-2, and PS1HL9 to $\mathrm{Pb}^{2+}$-bound DREAM $(\Delta 64)$ were probed by an increase in anisotropy. The fraction of site-1, site-2, and PS1HL9 bound to $\mathrm{Pb}^{2+}$-bound DREAM $(\Delta 64)$ were calculated using Equation 1. Dissociation constants between the titration of $\mathrm{Pb}^{2+} \operatorname{DREAM}(\Delta 64)$ and site-1, site-2, and PS1HL9 were determined by using a single-site binding model, Equation 2. All experiments were repeated three times; average $K_{d}$ values determined from 3 independent measurements are reported in Table 4, and the standard deviations are shown as errors.

\subsection{Methods used in chapter 4}

\subsubsection{General}

$\mathrm{CdCl}_{2}$ and 1-anilinonaphthalene- 8-sulfonic acid (1,8-ANS) were purchased from Sigma-Aldrich and Invitrogen, respectively, and used without further purification. $\mathrm{CdCl}_{2}$ and 1,8-ANS stocks were prepared in $20 \mathrm{mM}$ Tris $\mathrm{pH} 7.4$ using decalcified water. The synthetic peptide corresponding to the helix-9 of presenilin1 (PS1HL9), residues 445FYFATDYLVQPFMDQLAFHQFYI467 of the human presenilin-1 C-terminal fragment with FITC covalently attached to the aminoterminus was purchased from ProImmune (Sarasota, FL, USA). A FITC labeled $\begin{array}{lllll}\text { peptides corresponding to } & \text { residues } 22 & \text { (FITC- }\end{array}$ AAGVAAWLPFARAAAIGWMPV) and residues 70-90 (FITC- 
LLGSTEKEFFFNEDTKEYFFD) of human Kv4.3 and labeled site 1 and site 2, respectively, were obtained from Think Peptides and received at more than $98 \%$ purity. Unless noted otherwise, all other chemicals were purchased from SigmaAldrich or Fisher Scientific.

2.2.2 Isolation and purification of DREAM constructs

Expressions and purifications of recombinant mouse WTDREAM $(\Delta 64)$ (residue 65-256), WTDREAM $(\Delta 160)$ (residue 161-256), DREAM(E186Q) (residue 65-256 with mutation at position 12 in the loop of EF3), and DREAM(E234Q) (residue 65-256 with mutation at position 12 in the loop of EF4) constructs were carried out following published protocols (Osawa, Tong et al. 2001, Gonzalez, Pham et al. 2014). The purified protein stock solutions were stored in 20 $\mathrm{mM}$ Tris $\mathrm{pH}$ 7.4. SDS-PAGE was employed for assessing the purity of all DREAM constructs.

\subsubsection{Sample preparation}

Protein samples were prepared diluting concentrated protein stock in 20 $\mathrm{mM}$ Tris $\mathrm{pH} 7.4$ and final protein concentrations were determined using $\varepsilon_{280 \mathrm{~nm}}=$ $19,000 \mathrm{M}^{-1} \mathrm{~cm}^{-1}$ for WTDREAM ( $\left.\triangle 64\right)$, DREAM(E186Q) and DREAM(E234Q) and using $\varepsilon_{280 \mathrm{~nm}}=10,544 \mathrm{M}^{-1} \mathrm{~cm}^{-1}$ for WTDREAM $(\Delta 160)$. The concentrations of $1,8-$ ANS was determined using $\varepsilon_{350 \mathrm{~nm}}=4,999 \mathrm{M}^{-1} \mathrm{~cm}^{-1}$, whereas concentrations of site1, site-2, and PS1HL9 were determined using $\varepsilon_{493 \mathrm{~nm}}=75,000 \mathrm{M}^{-1} \mathrm{~cm}^{-1}$. To prepare ApoDREAM samples, protein stock was solubilized in $20 \mathrm{mM}$ Tris $\mathrm{pH} 7.4 .2 \mathrm{mM}$ EDTA was added to prepare metal-free samples. $\mathrm{Ca}^{2+}$ or $\mathrm{Cd}^{2+} \mathrm{DREAM}$ samples were prepared by adding $2 \mathrm{mM} \mathrm{Ca}{ }^{2+}$ and $100 \mu \mathrm{M} \mathrm{Cd}^{2+}$, respectively. $\mathrm{Cd}^{2+} \mathrm{Ca}^{2+} \mathrm{DREAM}$ 
was prepared by adding $100 \mu \mathrm{M} \mathrm{Cd}^{2+}$ to DREAM in the presence of $2 \mathrm{mM} \mathrm{Ca}^{2+}$. For the titration measurements, concentrated stocks of site-1, site-2, and PS1HL9 were diluted in $20 \mathrm{mM}$ Tris $\mathrm{pH} 7.4$ to have the final concentration of $0.5 \mu \mathrm{M}$.

2.2.4 Steady-state emission spectra

Steady-state emission spectra were recorded at room temperature $\left(20^{\circ} \mathrm{C}\right)$ using Cary Eclipse Fluorescence Spectrophotometer (Agilent Technologies). Tryptophan emission spectra were measured using $295 \mathrm{~nm}$ excitation along the 0.2 $\mathrm{cm}$ path of a $0.2 \times 1.0 \mathrm{~cm}$ path length quartz cuvette, 1,8-ANS emission spectra were measured using $350 \mathrm{~nm}$ excitation light along the $0.2 \mathrm{~cm}$ path of a $0.2 \times 1.0 \mathrm{~cm}$ path length quartz cuvette. The emission spectra were normalized to 1 at the maximum emission wavelength for the apoprotein in Fig. 2 and for Ca2+DREAM:1,8-ANS complex in Figure 4. The equilibrium dissociation constant for 1,8-ANS association to $\mathrm{Cd}^{2+}$-bound DREAM was obtained by titrating $\mathrm{Cd}^{2+}$-bound WTDREAM $(\Delta 64)$ into $10 \mu \mathrm{M}$ 1,8-ANS. The increase in emission intensity at an emission maximum of $468 \mathrm{~nm}$ was recorded and data were fitted to the single-site binding model according to Equation 1.

$\Delta \mathrm{F}=\frac{\left(\mathrm{K}_{\mathrm{d}}+\left[\mathrm{nP}_{\mathrm{t}}\right]+\left[\mathrm{L}_{\mathrm{t}}\right]\right)-\sqrt{\left(\mathrm{K}_{\mathrm{d}}+\left[\mathrm{nP}_{\mathrm{t}}\right]+\left[\mathrm{L}_{\mathrm{t}}\right]\right)^{2}-4\left[\mathrm{nP}_{\mathrm{t}}\right]\left[\mathrm{L}_{\mathrm{t}}\right]}}{\left[\mathrm{nP}_{\mathrm{t}}\right]}$

where $\Delta \mathrm{F}$ is the change in fluorescence intensity, $\mathrm{K}_{\mathrm{d}}$ is the equilibrium dissociation constant, $\mathrm{n}$ is the number of binding sites, $\mathrm{P}_{\mathrm{t}}$ is the total concentration of the protein, and $\mathrm{L}_{\mathrm{t}}$ is the total concentration of the ligand. Three independent measurements were carried out to determine the average value of $\Delta \mathrm{F}$. 


\subsubsection{Circular dichroism}

Far-UV circular dichroism measurements were conducted at room temperature $\left(20^{\circ} \mathrm{C}\right)$ using a J-810 Jasco CD spectrometer through the $2 \mathrm{~mm}$ path of a (2 mm x $10 \mathrm{~mm})$ quartz cuvette. Data were collected from 200 to $250 \mathrm{~nm}$. Secondary structure prediction tool K2D3 was used for calculating the percentage of secondary structure content of DREAM constructs (Louis-Jeune, Andrade-Navarro et al. 2012).

\subsubsection{Fluorescence lifetime measurements}

Frequency-domain fluorescence lifetime measurements were carried out at $20^{\circ} \mathrm{C}$ using a Chronos ${ }^{\mathrm{FD}}$ Spectrofluorometer (ISS, Champaign IL). The fluorescence lifetime of 1,8-ANS-WTDREAM( $\Delta 64)$ complexes were determined using the 305 $\mathrm{nm}$ laser diode output as excitation light and emission was recorded using the combination of a $400 \mathrm{~nm}$ long-pass filter and a $600 \mathrm{~nm}$ short-pass filter (Andover Corp., Salem, NH). To measure Trp lifetimes, samples were excited with $280 \mathrm{~nm}$ laser diode output and emission was recorded using a $320 \mathrm{~nm}$ long-pass filter. 1,4bis(5-phenyloxazol-2-yl)benzene (POPOP) in ethanol was used as a reference for lifetime measurements $(\tau=1.35 \mathrm{~ns})$. The lifetime modulation-phase data were analyzed using Vinci analysis software. The goodness of the fit was determined based on $\chi^{2}$ values and residuals.

\subsubsection{Thermodynamics of $\mathrm{Cd}^{2+}$ displacement of $\mathrm{Ca}^{2+}$ from EF-hand of DREAM}

Thermodynamic parameters for $\mathrm{Cd}^{2+}$ association to DREAM were determined using a VP-ITC isothermal calorimeter (Microcal Inc. Northampton, MA). DREAM protein stock in $20 \mathrm{mM}$ Tris was diluted to prepare $10 \mu \mathrm{M}$ DREAM 
protein solution in $5 \mathrm{mM}$ Tris $\mathrm{pH} 7.4,100 \mathrm{mM} \mathrm{NaCl}$ and $100 \mu \mathrm{M} \mathrm{CaCl}_{2}$ and dialyzed overnight three times against the buffer described above. After dialysis, the concentration of protein solution was determined and the protein solution was loaded in the reaction cell. $1 \mathrm{mM} \mathrm{Cd}^{2+}$ stock solution prepared in the dialysate buffer was used as the titrant. ITC experiments were performed three times at $25{ }^{\circ} \mathrm{C}$ by adding 30 aliquots $\left(6 \mu \mathrm{L}\right.$ each) of $1 \mathrm{mM} \mathrm{Cd}^{2+}$ stock into $10 \mu \mathrm{M}$ WTDREAM( $(\Delta 64)$ and $10 \mu \mathrm{M}$ WTDREAM $(\Delta 160)$. Data were fitted with the sequential model (2 sets of sites) using the Microcal ITC analysis plug-in Origin 7.0 The overall apparent dissociation constant $\left(\mathrm{K}_{\mathrm{app}}\right)$ for $\mathrm{Cd}^{2+}$ binding to $\mathrm{Ca}^{2+} \mathrm{DREAM}$ was calculated based on individual association constants according to Equation 2 (Aravind, Chandra et al. 2008).

$$
\mathrm{K}_{\mathrm{app}}=\frac{1}{\sqrt{\mathrm{K}_{1} \mathrm{~K}_{2}}}
$$

where $\mathrm{K}_{1}$ and $\mathrm{K}_{2}$ are apparent association constants for $\mathrm{Cd}^{2+}$ binding to $\mathrm{Ca}^{2+}$ DREAM.

The overall equilibrium dissociation constant $\left(\mathrm{K}_{\mathrm{d}}\right)$ for $\mathrm{Cd}^{2+}$ binding to apoDREAM was calculated from the overall apparent dissociation constant using Equation 3 (Kirberger, Wong et al. 2013).

$$
\mathrm{K}_{\mathrm{d}}=\frac{\mathrm{K}_{\mathrm{app}} \mathrm{K}_{\mathrm{dCa}}{ }^{2+}}{\left(\mathrm{KdCa}^{2+}+\left[\mathrm{Ca}^{2+}\right] \mathrm{T}\right)}
$$

where $\mathrm{K}_{\mathrm{d}_{\mathrm{Ca}}{ }^{2+}}$ represents the equilibrium dissociation constant for $\mathrm{Ca}^{2+}$ binding to apoDREAM, which was previously reported to be $1 \mu \mathrm{M}$ (Osawa, Dace et al. 2005); $\left[\mathrm{Ca}^{2+}\right]_{\mathrm{T}}$ is the total concentration of $\mathrm{Ca}^{2+}$, which is $100 \mu \mathrm{M}$. 
2.2.8 Steady-state anisotropy measurements

The steady-state anisotropy measurements were conducted at room temperature $\left(20^{\circ} \mathrm{C}\right)$ using a Chronos ${ }^{\mathrm{FD}}$ spectrofluorometer (ISS, Champaign IL). To investigate whether FITC-tagged PS1HL9, FITC-tagged site-1, or FITC-tagged site2 binds to $\mathrm{Cd}^{2+}$-bound DREAM, $\mathrm{Cd}^{2+}$-bound DREAM constructs were titrated into $0.5 \mu \mathrm{M}$ PS1HL9, $0.5 \mu \mathrm{M}$ site-1, or $0.5 \mu \mathrm{M}$ site-2 peptides. Samples were excited at $470 \mathrm{~nm}$ and emitted light was recorded through a $500 \mathrm{~nm}$ long-pass filter (Andover Corp., Salem, NH). Three independent measurements were carried out and average values of anisotropy change were plotted as a function of $\mathrm{Cd}^{2+} \mathrm{DREAM}$ concentration and analyzed using Equation 1.

\subsection{Methods used in chapter 5}

\subsubsection{General}

The $\mathrm{LiCl}$ was purchased from Sigma-Aldrich and used as received. $100 \mathrm{mM} \mathrm{LiCl}$ stock was prepared by dissolving $\mathrm{LiCl}$ in $20 \mathrm{mM}$ Tris buffer $\mathrm{pH}$ 7.4. To eliminate residual $\mathrm{Ca}^{2+}, 20 \mathrm{mM}$ Tris buffer was prepared in decalcified ultrapure $18 \mathrm{M} \Omega$ deionized water. Peptides with a sequence corresponding to the site- 1 and site- 2 binding sites from $\mathrm{T} 1$ domain of $\mathrm{Kv}$ channel with a FITC fluorescence probe attached to the $\mathrm{N}$ - terminus (FITCsite-1 of human $\mathrm{K}_{\mathrm{V}} 4.3$ ) (FITC-AAGVAAWLPFARAAAIGWMPV) and (FITC-site-2 of human $\mathrm{K}_{\mathrm{V}} 4.3$ ) (FITC-LLGSTEKEFFFNEDTKEYFFD) were purchased from Think Peptides and received at higher than $98 \%$ purity. The synthetic peptide derived for helix9 of presenilin-1 (FITC- ${ }^{445}$ FYFATDYLVQPFMDQLAFHQFYI ${ }^{467}$ ) of human PS1-CTF was purchased from ProImmune (Sarasota, FL, USA). All other chemicals were purchased from Sigma-Aldrich or Thermo Fisher unless stated otherwise. The concentration of 
peptides mimicking site-1, site-2, and helix-9 of presenilin-1 were measured by using $\varepsilon_{493 \mathrm{~nm}}=75000 \mathrm{M}^{-1} \mathrm{~cm}^{-1}$ (Crabtree, Shammas 2018).

\subsubsection{Isolation and purification of DREAM constructs}

In the present study, a truncated form of DREAM protein that carries residues 65256 (DREAM $(\Delta 64))$ was used, as the full-length protein readily forms inclusion bodies.

Recombinant mouse $\operatorname{DREAM}(\Delta 64), \quad \operatorname{DREAM}(\Delta 160), \quad \operatorname{DREAM}(\mathrm{E} 168 \mathrm{Q}), \quad$ and DREAM(E234Q) constructs were expressed in E. coli BL21 (DE3) cells and purified according to published procedures(Osawa, Tong et al. 2001, Gonzalez, Pham et al. 2014). Briefly, corresponding mouse DREAM plasmid was transfected in E. coli BL21(DE3) competent cells and grown in Terrific Broth medium at $37^{\circ} \mathrm{C}$ in the presence of $100 \mu \mathrm{g} / \mathrm{mL}$ ampicillin. Protein expression was induced by adding $0.5 \mathrm{mM}$ IPTG. Cells were grown for an additional 12 hours at room temperature and harvested by 20 min centrifugation at 5000 RPM and $4{ }^{\circ} \mathrm{C}$. The cell pellet was resuspended in lysis buffer $(100 \mathrm{mM}$ Tris buffer $\mathrm{pH}$ 8.0, $0.5 \mathrm{M} \mathrm{NaCl}, 1 \mathrm{mM} \mathrm{CaCl}, 5 \mathrm{mM} \mathrm{MgCl}, 5 \mathrm{mM}$ imidazole, $1.0 \%$ Triton-X100, $0.5 \%$ Tween 20, and 10\% glycerol, $20 \mu \mathrm{g} / \mathrm{mL}$ DNAse, and $200 \mu \mathrm{g} / \mathrm{mL}$ lysozyme) and sonicated in an ice bath for 30 cycles at $30 \%$ duty cycle. The cell lysate was centrifuged at $5000 \mathrm{rpm}$ for 4 hours and the supernatant was loaded to a Ni-NTA column equilibrated with buffer A (20 mM Tris $\mathrm{pH} 7.4,300 \mathrm{mM} \mathrm{NaCl}, 0.5 \mathrm{mM} \mathrm{CaCl}_{2}$, and $5 \mathrm{mM}$ imidazole). The Ni-NTA column was loaded with DREAM and then washed extensively with buffer A and increasing concentration of imidazole $(5-40 \mathrm{mM})$ and protein was eluted in buffer A and $250 \mathrm{mM}$ imidazole. Selected fractions were loaded onto a DEAE-Sepharose anion exchange column previously equilibrated with $20 \mathrm{mM}$ Tris $\mathrm{pH}$ 7.4, washed with $20 \mathrm{mM}$ Tris pH 7.4 and $50 \mathrm{mM} \mathrm{NaCl}$, and then eluted with $20 \mathrm{mM}$ Tris, pH 7.4 and $200 \mathrm{mM} \mathrm{NaCl}$. 
After anion exchange, the protein was dialyzed twice against two liters of $20 \mathrm{mM}$ Tris $\mathrm{pH}$ 7.4 for 12 hours. The purified protein was stored in $20 \mathrm{mM}$ Tris $\mathrm{pH}$ 7.4. The purity of all protein constructs was assessed using SDS-PAGE. Protein concentration was determined by UV-VIS spectrophotometry using $\varepsilon_{280 \mathrm{~nm}}=19,000 \mathrm{M}^{-1} \mathrm{~cm}^{-1}$ for DREAM $(\Delta 64)$ and $\varepsilon_{280 \mathrm{~nm}}$ $=10,544 \mathrm{M}^{-1} \mathrm{~cm}^{-1}$ for DREAM ( $\left.\Delta 160\right)$ (Pham, Dhulipala et al. 2015). Apoprotein samples were prepared by dialyzing the isolated protein three times against $20 \mathrm{mM}$ Tris buffer that was prepared using decalcified water. The absence of $\mathrm{Ca}^{2+}$ bound DREAM in apoDREAM samples was confirmed based on the emission spectra of apoDREAM in the absence/presence of EDTA. $\mathrm{Li}^{+}$DREAM samples were prepared by adding $50 \mu \mathrm{M}$ or 1 $\mathrm{mM} \mathrm{LiCl}$ to $20 \mu \mathrm{M}$ protein in $20 \mathrm{mM}$ Tris $\mathrm{pH} 7.4, \mathrm{Mg}^{2+}$ DREAM samples were prepared by the addition of $5 \mathrm{mM} \mathrm{MgCl}_{2}$ and $1 \mathrm{mM}$ EGTA to $20 \mu \mathrm{M}$ protein in $20 \mathrm{mM}$ Tris $\mathrm{pH} 7.4$, and $\mathrm{Ca}^{2+}$ DREAM samples were prepared by adding $1 \mathrm{mM} \mathrm{CaCl}_{2}$ to $20 \mu \mathrm{M}$ protein in 20 $\mathrm{mM}$ Tris $\mathrm{pH} 7.4$.

\subsubsection{Isolation and purification of $\mathrm{rCaM}$}

Rat calmodulin (rCaM) plasmid was a kind gift from J. P. Davis (The Ohio State University, Columbus, OH). The plasmid was subcloned in BL21(DE3) cells and purified using phenyl sepharose chromatography as previously described (George, Su et al. 1993). 2.3.4 Steady-state emission spectra measurements

Emission spectra for a single Trp residue in DREAM constructs were measured in a $0.2 \times 1-\mathrm{cm}$ path length quartz cuvette with $295 \mathrm{~nm}$ excitation along the $0.2-\mathrm{cm}$ path using a custom Chronos ${ }^{\mathrm{FD}}$ spectrofluorometer (ISS, Champaign, IL). The concentration of DREAM constructs was $20 \mu \mathrm{M}$. The equilibrium dissociation constant for $\mathrm{Li}^{+}$binding to 
apoDREAM was determined by adding small aliquots of $\mathrm{Li}^{+}$stock solution to $20 \mu \mathrm{M}$ apoDREAM $(\Delta 64)$ in $20 \mathrm{mM}$ Tris buffer $\mathrm{pH}$ 7.4.

The apparent equilibrium dissociation constant, $\mathrm{K}_{\mathrm{d}}$ was obtained according to Eq.1.

$\Delta \mathrm{F}=\frac{\left(\mathrm{K}_{\mathrm{d}}+\left[\mathrm{nP}_{\mathrm{t}}\right]+\left[\mathrm{L}_{\mathrm{t}}\right]\right)-\sqrt{\left(\mathrm{K}_{\mathrm{d}}+\left[\mathrm{nP}_{\mathrm{t}}\right]+\left[\mathrm{L}_{\mathrm{t}}\right]\right)^{2}-4\left[\mathrm{nP}_{\mathrm{t}}\right]\left[\mathrm{L}_{\mathrm{t}}\right]}}{\mathrm{n} P_{t}}$

where $\mathrm{K}_{\mathrm{d}}$ is the dissociation constant, $\mathrm{P}_{\mathrm{t}}$ is total protein concentration, $\mathrm{n}$ is the number of binding sites, $\mathrm{L}_{t}$ is the total ligand concentration. The experiment was repeated three times and the average fluorescence intensity values were used to construct the titration curve.

2.3.4 Circular dichroism measurements

Far UV-CD spectra were recorded using a J-810 Jasco CD spectrometer. $20 \mu \mathrm{M}$ DREAM in $20 \mathrm{mM}$ Tris buffer, $\mathrm{pH} 7.4$ and $1 \mathrm{mM} \mathrm{Ca}^{2+}$ and/or $50 \mu \mathrm{M} \mathrm{Li}^{+}$, were placed in a quartz cuvette $(1 \mathrm{~mm} \times 10 \mathrm{~mm})$ and data were collected from $200 \mathrm{~nm}$ to $250 \mathrm{~nm}$.

\subsubsection{ITC measurements}

Isothermal calorimetry titrations were used to determine the thermodynamics of $\mathrm{Li}^{+}$ binding to DREAM constructs. Titrations were carried out using a VP-ITC isothermal calorimeter (Microcal Inc. Northampton, MA). Protein solutions were dialyzed against 5 $\mathrm{mM}$ Tris, $\mathrm{pH}$ 7.4, prepared in decalcified water for 12 hours. Water was decalcified using Chelex-100 . The buffer was exchanged three times during the dialysis. $100 \mathrm{mM} \mathrm{Li}^{+}$stock solution was prepared by dissolving $\mathrm{LiCl}$ in an ITC dialyzate buffer. The reaction cell was loaded with $\sim 20 \mu \mathrm{M}$ protein solution in $5 \mathrm{mM}$ Tris, $\mathrm{pH}$ 7.4. The concentration of $\mathrm{Li}^{+}$in the syringe was $1000 \mu \mathrm{M} ; 30$ aliquots ( $6 \mu \mathrm{L}$ each) of $\mathrm{Li}^{+}$stock solutions were titrated into 20

$\mu \mathrm{M}$ DREAM constructs at $25{ }^{\circ} \mathrm{C}$. Three independent ITC experiments were conducted. Thermodynamic parameters were obtained by fitting the isotherms with the sequential 
model (two sets of sites) using the Microcal ITC analysis plug-in Origin 7.0. Overall dissociation constant was obtained from individual association constants by using Equation 2 (Aravind, Chandra et al. 2008).

$\mathrm{K}_{\mathrm{d}}=\frac{1}{\sqrt{\mathrm{K}_{1} \mathrm{~K}_{2}}}$

where $\mathrm{K}_{\mathrm{d}}$ represents overall dissociation constant for $\mathrm{Li}^{+}$binding to apoDREAM constructs, whereas $\mathrm{K}_{1}$ and $\mathrm{K}_{2}$ represent association constants of $\mathrm{Li}^{+}$binding to apoDREAM constructs.

To determine whether $\mathrm{Li}^{+}$binds to $\mathrm{Ca}^{2+}$ DREAM, we titrated 30 aliquots $(6 \mu \mathrm{L}$ each) of $100 \mathrm{mM} \mathrm{Li}^{+}$into $20 \mu \mathrm{M}$ DREAM( $(\Delta 64)$ in the presence of $100 \mu \mathrm{M} \mathrm{Ca}^{2+}$ at $25^{\circ} \mathrm{C}$.

\subsubsection{Molecular Dynamics}

The protein structure of DREAM was taken from the Protein Data Bank (PDB ID: 2JUL) (Lusin, Vanarotti et al. 2008). We considered residues 76 to 256 of the DREAM monomer for the molecular simulations. The CHARMM-GUI web-server was used to set up the system for molecular dynamics simulation (Jo, Kim et al. 2008). The $\mathrm{Ca}^{2+}$ ions bound to the protein in the crystal structure were removed and lithium ions were placed randomly in the solution. The system was solvated using TIP3 water molecules and neutralized by counterions. All-atom molecular dynamics simulations were performed using NAMD 2.12 with CHARMM36m force field at $300 \mathrm{~K}$ temperature (Phillips, Braun et al. 2005). The system was minimized for 10,000 steps and equilibrated for $200 \mathrm{ps}$ with backbone and side chain restraints, followed by a 200-ns production run using 2 fs time step. The SHAKE algorithm was used for constraining covalent bonds and particle mesh Ewald method was employed for long-range electrostatic interactions. Langevin 
temperature coupling with a friction coefficient of $1 \mathrm{ps}$ was used to control the temperature. Analysis of the trajectory as well as visualization of accessible surface area was done with VMD (Humphrey, Dalke et al. 1996).

2.3.7 Fluorescence lifetime measurements

Frequency-domain fluorescence 1,8-ANS lifetime measurements were performed using a Chronos ${ }^{\mathrm{FD}}$ spectrofluorometer (ISS, Champaign IL). Samples were excited using an output of $300 \mathrm{~nm}$ laser diode and emission was collected through a combination of $400 \mathrm{~nm}$ long-pass filter and $600 \mathrm{~nm}$ short-pass filter (Andover Corp.). 1,4bis(5-phenyloxazol-2-yl) benzene (POPOP) in ethanol was used as a reference in lifetime measurements $(\tau=1.35 \mathrm{~ns})$. Experimental data were fitted by using a three exponential decay model in Vinci software (ISS, Champaign IL). The $\chi^{2}$ parameters and residuals were used as a criterion for the goodness of the fit.

\subsubsection{Steady-state polarization measurements}

The steady-state polarization measurements were carried out in a Chronos ${ }^{\mathrm{FD}}$ spectrofluorometer (ISS, Champaign IL). To investigate the interaction between $\operatorname{DREAM}(\Delta 64)$ and FITC-labeled peptides, samples were excited at $493 \mathrm{~nm}$ and the emitted light was collected through a $500 \mathrm{~nm}$ long pass filter (Andover Corp). For anisotropy measurements, $20 \mu \mathrm{M}$ DREAM was added to $0.5 \mu \mathrm{M}$ FITC labeled peptides in the absence/presence of $1 \mathrm{mM} \mathrm{Li}^{+}$or $1 \mathrm{mM} \mathrm{Ca}^{2+}$. The presented anisotropy values represent the average values of 3 independent measurements. 


\subsection{Methods used in chapter 6}

\subsubsection{General}

Barium chloride, strontium chloride, arsenic pentoxide, and mercuric oxide were purchased from Sigma-Aldrich. Manganese chloride tetrahydrate was purchased from Fisher Scientific. 1,8-ANS was purchased from Invitrogen.

\subsubsection{Isolation and purification of DREAM}

Recombinant mouse $\operatorname{DREAM}(\Delta 64)$ was expressed and purified following published protocols (Osawa, Tong et al. 2001, Gonzalez, Pham et al. 2014). SDSPAGE gel electrophoresis was used for evaluating the purity of the protein.

\subsubsection{Sample preparation}

Concentrated DREAM $(\Delta 64)$ protein stock was diluted into $20 \mathrm{mM}$ Tris $\mathrm{pH}$ 7.4 to get $20 \mu \mathrm{M}$ DREAM( $\Delta 64)$. Protein concentration was determined using $\varepsilon_{280 \mathrm{~nm}}$ $=19,000 \mathrm{M}^{-1} \mathrm{~cm}^{-1} \cdot 1,8$-ANS concentration was determined using $\varepsilon_{350 \mathrm{~nm}}=4,999$ $\mathrm{M}^{-1} \mathrm{~cm}^{-1} .1 \mathrm{mM}$ of $\mathrm{Ba}^{2+}, \mathrm{Sr}^{2+}, \mathrm{As}^{5+}, \mathrm{Hg}^{2+}$, or $\mathrm{Mn}^{2+}$ was added to $\operatorname{DREAM}(\Delta 64)$ or $\operatorname{DREAM}(\Delta 64): 1,8$-ANS complex both in the absence and presence of $1 \mathrm{mM} \mathrm{Ca}^{2+}$ to investigate impact of these metals addition on Trp emission and 1,8-ANS emission spectrum.

2.4.4 Steady-state emission spectra

Steady-state emission experiment was conducted at room temperature $\left(20^{\circ} \mathrm{C}\right)$ employing Cary Eclipse Fluorescence Spectrophotometer (Agilent Technologies). Samples were excited with $295 \mathrm{~nm}$ excitation light through the 0.2 $\mathrm{cm}$ path of a $0.2 \times 1.0 \mathrm{~cm}$ path length quartz cuvette to obtain tryptophan emission spectra.1,8-ANS emission spectra were obtained by exciting the samples with 350 
$\mathrm{nm}$ excitation light through the $0.2 \mathrm{~cm}$ path of a $0.2 \times 1.0 \mathrm{~cm}$ path length quartz cuvette.

\subsection{Methods used in chapter 7}

2.5.1 Isolation and purification of $\operatorname{DREAM}(\mathrm{K} 87 \mathrm{~A})$

Expressions and purifications of recombinant mouse DREAM(K87A) were executed following published protocols (Osawa, Tong et al. 2001, Gonzalez, Pham et al. 2014). Concentrated protein stock solutions were stored in $20 \mathrm{mM}$ Tris $\mathrm{pH}$ 7.4. The purity of the protein was assayed by SDS-PAGE gel electrophoresis.

\subsubsection{Sample preparation}

Protein samples were prepared by diluting concentrated stock in $20 \mathrm{mM}$ Tris pH 7.4 and final protein concentrations were determined using $\varepsilon_{280 \mathrm{~nm}}=19,000 \mathrm{M}^{-}$ ${ }^{1} \mathrm{~cm}^{-1}$. The concentrations of 1,8 -ANS was determined using $\varepsilon_{350 \mathrm{~nm}}=4,999 \mathrm{M}^{-1} \mathrm{~cm}^{-1}$, whereas concentrations of site-1, site-2, and PS1HL9 were determined using $\varepsilon_{493 \mathrm{~nm}}$ $=75,000 \mathrm{M}^{-1} \mathrm{~cm}^{-1}$. For Trp emission, 1,8-ANS study, CD study, and lifetime studies, the concentration of DREAM(K87A) was $20 \mu \mathrm{M}$. apoDREAM(K87A) was prepared by adding $1 \mathrm{mM}$ EDTA to protein solution. $\mathrm{Ca}^{2+} \mathrm{DREAM}(\mathrm{K} 87 \mathrm{~A})$ samples were prepared by adding $1 \mathrm{mM} \mathrm{Ca}^{2+}$ to protein solution in the absence of EDTA or EGTA. $\mathrm{Mg}^{2+} \mathrm{DREAM}(\mathrm{K} 87 \mathrm{~A})$ was prepared by adding $5 \mathrm{mM} \mathrm{Mg}^{2+}$ to protein solutions in the presence of $1 \mathrm{mM}$ EGTA. $\mathrm{Ca}^{2+} \mathrm{Mg}^{2+}$ DREAM(K87A) was prepared by adding $5 \mathrm{mM} \mathrm{Mg}^{2+}$ to $\operatorname{DREAM}(\mathrm{K} 87 \mathrm{~A})$ in the presence of $1 \mathrm{mM} \mathrm{Ca}^{2+}$. For the titration measurements, concentrated stocks of site-1, site-2, and PS1HL9 were diluted in $20 \mathrm{mM}$ Tris $\mathrm{pH} 7.4$ to get the final concentration of $0.5 \mu \mathrm{M}$. 


\subsubsection{Steady-state emission spectra}

Cary Eclipse Fluorescence Spectrophotometer (Agilent Technologies) was employed at room temperature $\left(20^{\circ} \mathrm{C}\right)$ to record steady-state emission spectra. For tryptophan emission, samples were excited with $295 \mathrm{~nm}$ excitation light along the $0.2 \mathrm{~cm}$ path of a $0.2 \times 1.0 \mathrm{~cm}$ path length quartz cuvette. For 1,8 -ANS emission, samples were excited through $350 \mathrm{~nm}$ excitation light along the $0.2 \mathrm{~cm}$ path of a 0.2 $\mathrm{x} 1.0 \mathrm{~cm}$ path length quartz cuvette.

2.5.4 Circular dichroism

A J-810 Jasco CD spectrometer was employed at room temperature $\left(20^{\circ} \mathrm{C}\right)$ to measure far-UV circular dichroism measurements through the $2 \mathrm{~mm}$ path of a (2 $\mathrm{mm} \times 10 \mathrm{~mm}$ ) quartz cuvette. Data were recorded from 200 to $250 \mathrm{~nm}$. The percentage of secondary structure content was obtained using secondary structure prediction tool K2D3 (Louis-Jeune, Andrade-Navarro et al. 2012).

\subsubsection{Fluorescence lifetime measurements}

Frequency-domain fluorescence lifetime measurements were conducted at room temperature $\left(20^{\circ} \mathrm{C}\right)$ utilizing a Chronos $^{\mathrm{FD}}$ Spectrofluorometer (ISS, Champaign IL). The fluorescence lifetime of 1,8-ANS-DREAM(K87A) complexes were measured by exciting the samples through $305 \mathrm{~nm}$ laser diode output and emission was collected using a combination of a $400 \mathrm{~nm}$ long-pass filter and a 600 nm short-pass filter (Andover Corp., Salem, NH). For Trp lifetimes, $280 \mathrm{~nm}$ laser diode output was used to excite the samples and emission was recorded using a 320 $\mathrm{nm}$ long pass filter. 1,4-bis(5-phenyloxazol-2-yl)benzene (POPOP) in ethanol was the reference for lifetime measurements $(\tau=1.35 \mathrm{~ns})$. Vinci analysis software was 
employed to analyze the modulation-phase data. $\chi^{2}$ values and residuals of the fit was used to evaluate the goodness of the fit.

2.5.6 Steady-state anisotropy measurements

The steady-state anisotropy measurements were carried out at room temperature $\left(20^{\circ} \mathrm{C}\right)$ employing a Chronos ${ }^{\mathrm{FD}}$ spectrofluorometer (ISS, Champaign IL). To investigate whether mutation of Lys87 to Ala impacts DREAM interactions with binding partners, DREAM(K87A) samples were added to FITC-tagged peptide helix 9 of presenilin-1 (PS1HL9) and FITC-tagged site-1 and site-2 of T-1 domain of Kv4 channel. Samples were excited through $493 \mathrm{~nm}$ excitation light and emitted light was collected through a $500 \mathrm{~nm}$ long-pass filter (Andover Corp., Salem, NH). $200 \mu \mathrm{M}$ DREAM(K87A) in the presence of $1 \mathrm{mM} \mathrm{Ca}^{2+}$ or $1 \mathrm{mM}$ EDTA was added to $0.5 \mu \mathrm{M}$ PS1HL9. Corresponding wild-type DREAM (DREAM $(\Delta 64))$ was also added to the same peptide in the same day as a control experiment. Similarly, 100 $\mu \mathrm{M}$ and $200 \mu \mathrm{M}$ of DREAM(K87A) were respectively added to $0.5 \mu \mathrm{M}$ site 1 and site 2 peptides of the $\mathrm{T} 1$ domain of $\mathrm{Kv} 4$ channel in the presence of $\mathrm{Ca}^{2+}$ or EDTA. $\operatorname{DREAM}(\Delta 64)$ was added to both site 1 and site 2 peptides on the same day as a control.

\subsection{Methods used in chapter 8}

2.6.1 Isolation and purification of DREAM constructs

Recombinant mouse DREAM( $\Delta 64)$, DREAM( $\Delta 160)$, DREAM(E186Q), and DREAM(E234Q) were expressed and purified following published protocols (Osawa, Tong et al. 2001, Gonzalez, Pham et al. 2014). SDS-PAGE gel electrophoresis was employed for assessing the purity of the protein. 


\subsubsection{Sample preparation}

Concentrated protein stock was diluted into $20 \mathrm{mM}$ Tris $\mathrm{pH} 7.4$ to get the

desired concentration. For $\operatorname{DREAM}(\Delta 64), \quad \operatorname{DREAM}(\operatorname{E186Q}), \quad$ and $\operatorname{DREAM}(\mathrm{E} 234 \mathrm{Q})$, protein concentrations were determined using $\varepsilon_{280 \mathrm{~nm}}=19,000 \mathrm{M}^{-}$ ${ }^{1} \mathrm{~cm}^{-1}$. For $\operatorname{DREAM}(\Delta 160)$, protein concertation was determined using $\varepsilon_{280 \mathrm{~nm}}=$ $10,544 \mathrm{M}^{-1} \mathrm{~cm}^{-1}$. 1,8-ANS concentration was determined using $\varepsilon_{350 \mathrm{~nm}}=4,999$ $\mathrm{M}^{-1} \mathrm{~cm}^{-1}$; site-1 and site-2 concentrations were determined using $\varepsilon_{493 \mathrm{~nm}}=75,000$ $\mathrm{M}^{-1} \mathrm{~cm}^{-1}$. For Trp emission, 1,8-ANS emission, circular dichroism, and lifetime studies, the concentration of each DREAM protein construct was $20 \mu \mathrm{M}$. apoDREAM samples were prepared by adding $1 \mathrm{mM}$ EDTA to protein solutions that were stored in $20 \mathrm{mM}$ Tris. $\mathrm{Ca}^{2+}$ DREAM samples were prepared by adding 1 $\mathrm{mM} \mathrm{Ca}^{2+}$ to protein solutions that were stored in $20 \mathrm{mM}$ Tris. For the titration measurements, $0.5 \mu \mathrm{M}$ site-1, site-2, and PS1HL9 were prepared by diluting concentrated stock into $20 \mathrm{mM}$ Tris $\mathrm{pH} 7.4$.

2.6.3 Steady-state emission spectra

Steady-state emission spectra were obtained at room temperature $\left(20^{\circ} \mathrm{C}\right)$ utilizing Cary Eclipse Fluorescence Spectrophotometer (Agilent Technologies). Samples were excited through $295 \mathrm{~nm}$ excitation light through the $0.2 \mathrm{~cm}$ path of a $0.2 \times 1.0 \mathrm{~cm}$ path length quartz cuvette to obtain tryptophan emission spectra. Samples were excited with $350 \mathrm{~nm}$ excitation light through the $0.2 \mathrm{~cm}$ path of a 0.2 x $1.0 \mathrm{~cm}$ path length quartz cuvette to obtain $1,8-\mathrm{ANS}$ emission spectra. 


\subsubsection{Circular dichroism}

Far-UV circular dichroism spectra were recorded employing a J-810 Jasco CD spectrometer through the $2 \mathrm{~mm}$ path of a $(2 \mathrm{~mm} \times 10 \mathrm{~mm})$ quartz cuvette. Data were collected from 200 to $250 \mathrm{~nm}$. Secondary structure prediction tool K2D3 was used to determine the percentage of secondary structural content of the protein. (Louis-Jeune, Andrade-Navarro et al. 2012).

2.6.5 Fluorescence lifetime measurements

Frequency-domain fluorescence lifetime measurements were recorded at room temperature $\left(20^{\circ} \mathrm{C}\right)$ employing a Chronos ${ }^{\mathrm{FD}}$ Spectrofluorometer (ISS, Champaign IL). The fluorescence lifetime of 1,8-ANS-DREAM complexes were measured by exciting the samples with $305 \mathrm{~nm}$ laser diode output and emission were recorded using a combination of a $400 \mathrm{~nm}$ long-pass filter and a $600 \mathrm{~nm}$ short-pass filter (Andover Corp., Salem, NH). A $280 \mathrm{~nm}$ laser diode output was used to excite the samples and emission was recorded using a $320 \mathrm{~nm}$ long-pass filter for determining the lifetime of tryptophan. The reference for lifetime measurements was 1,4-bis(5-phenyloxazol-2-yl)benzene (POPOP) in ethanol $(\tau=1.35 \mathrm{~ns})$. Vinci

analysis software was utilized to analyze the modulation-phase data. $\chi^{2}$ values as well as residuals of the fit was used to evaluate the goodness of the fit. 2.6.6 Steady-state anisotropy measurements

The steady-state anisotropy measurements were conducted at room temperature $\left(20^{\circ} \mathrm{C}\right)$ utilizing a Chronos ${ }^{\mathrm{FD}}$ spectrofluorometer (ISS, Champaign IL). $100 \mu \mathrm{M}$ DREAM(E186Q) and $100 \mu \mathrm{M}$ DREAM(E234Q) samples were added to FITC-tagged site- 1 and site- 2 of the T- 1 domain of Kv 4 channel both in the presence 
and absence of $\mathrm{Ca}^{2+}$. Samples were excited with $493 \mathrm{~nm}$ excitation light and emitted light was collected through a $500 \mathrm{~nm}$ long-pass filter (Andover Corp., Salem, NH). Corresponding $100 \mu \mathrm{M}$ wild-type DREAM (DREAM $(\Delta 64)$ ) was also added to the same peptide in the same day as a control experiment both in the presence and absence of $\mathrm{Ca}^{2+}$. 


\section{LEAD BINDS TO DREAM AND MODULATES ITS INTERACTIONS WITH BINDING PARTNERS: A LINK BETWEEN NEURONAL CALCIUM SENSORS AND LEAD NEUROTOXICITY.}

\subsection{Background and significance}

$\mathrm{Pb}^{2+}$ is an anthropogenic toxicant and has been viewed as a global health concern (Deibler, Basu 2013). The chronic and acute exposure to $\mathrm{Pb}^{2+}$ cause neurophysiological and psychological deficiencies through altering the development of the neuronal system or through direct interactions with neuronal proteins (Mason, Harp et al. 2014). Especially children are sensitive to $\mathrm{Pb}^{2+}$ exposure as $\mathrm{Pb}^{2+}$ intoxication leads to learning difficulties, IQ drop and cognitive deficiencies (Canfield, Henderson Jr et al. 2003, Weisskopf, Hu et al. 2004). Several metal-binding proteins have been identified as targets for $\mathrm{Pb}^{2+}$ neurotoxicity as $\mathrm{Pb}^{2+}$ displaces physiological ligands such as calcium and zinc; however, the mechanism of $\mathrm{Pb}^{2+}$ induced toxicity remains obscure. It was reported that at subnanomolar concentrations, $\mathrm{Pb}^{2+}$ activates protein kinase $\mathrm{C}(\mathrm{PKC})$, a family of proteins that mediate central nervous system development and cell proliferation, by replacing a $\mathrm{Ca}^{2+}$ ion bound to the C2 domain (Markovac, Goldstein 1988, Long, Rosen et al. 1994, Morales, Lasagna et al. 2011). $\mathrm{Pb}^{2+}$ was also shown to directly inhibit the constitutive kinase activity of PKC through binding to a lower affinity site in the catalytic domain. Several studies have demonstrated that $\mathrm{Pb}^{2+}$ interacts with calcium-binding protein calmodulin $(\mathrm{CaM})$, and modulate interactions between $\mathrm{CaM}$ and its downstream partners what may play a considerable role in $\mathrm{Pb}^{2+}$-induced neurotoxicity (Gorkhali, Huang et al. 2016). For example, Habermann et al.(Habermann, Crowell et al. 1983) reported that $\mathrm{Pb}^{2+}$ replaces $\mathrm{Ca}^{2+}$ bound to EF-hands in $\mathrm{CaM}$ and $\mathrm{Pb}^{2+}: \mathrm{CaM}$ complex activates calcium-dependent 
phosphodiesterase and myosin light chain kinase. $\mathrm{Pb}^{2+}$ can also bind to sites other than $\mathrm{EF}-$ hands, which may disrupt multiple downstream activities and therefore contribute to lead neurotoxicity (Kern, Wisniewski et al. 2000, Goldstein, Ar 1983). Statistical analysis of the structures of $\mathrm{Pb}^{2+}$-bound proteins available from the protein data bank (PDB) showed that an oxygen atom from amino acids (mainly Glu and Asp) represents the prominent binding ligand for $\mathrm{Pb}^{2+}$, followed by oxygen from water, sulfur, and nitrogen (Kirberger, Yang 2008). The basic geometry of $\mathrm{Ca}^{2+}$-bound EF-hand was found to be conserved in $\mathrm{Pb}^{2+}$-bound EF-hand, as both metals, $\mathrm{Ca}^{2+}$ and $\mathrm{Pb}^{2+}$, use the same binding ligands, (Kirberger, Yang 2008) suggesting that $\mathrm{Pb}^{2+}$ could tightly bind to EF-hands of other neuronal calcium-binding proteins (CaBPs). Indeed, the multitude of symptoms associated with $\mathrm{Pb}^{2+}$ poisoning points towards multiple molecular targets in neuronal tissue.

Neuronal Calcium Sensor (NCS) is a subgroup of EF-hand superfamily, which includes frequenin (NCS1), recoverin, GCAP, neurocalcin, and potassium channelinteracting protein 1-4 (Burgoyne 2007). NSC proteins are predominantly expressed in brain and retina, where they regulate diverse intracellular processes, including vision transduction, DNA expression, neurotransmission, neuron development, cognitive functions, potassium voltage channel kinetics, etc. (Ames, Lim 2012, Burgoyne, Weiss 2001, Burgoyne 2007). Although NCS proteins have relatively low sequence homology with CaM, NCS proteins comprise four EF-hands analogous to CaM. Similarly, NCS proteins are sensitive to increased intracellular $\mathrm{Ca}^{2+}$ concentration and bind $\mathrm{Ca}^{2+}$ with $\sim 10$ times higher affinity than CaM (McCue, Haynes et al. 2010).

Downstream regulatory element antagonist modulator (DREAM), alternatively known as calsenilin or potassium channel-interacting protein 3 (KChIP3), is a $29 \mathrm{kDa}$ NCS 
protein, which is involved in numerous biological processes, such as gene apoptosis, pain sensation, memory and learning processes, and modulation of Kv4 voltage channels expression and kinetics (An, Bowlby et al. 2000, Buxbaum, Choi et al. 1998, Carrion, Link et al. 1999). In the nucleus, it regulates the expression of prodynorphin and c-fos gene by binding to the DRE (downstream regulatory element) sequence in a calcium-dependent manner (Carrion, Link et al. 1999, Cheng, Pitcher et al. 2002). In the absence of $\mathrm{Ca}^{2+}$, DREAM associates to the DRE promoter region and consequently inhibits gene transcriptions. These genes have been found to be involved in cell homeostasis, apoptosis, and pain modulation (Costigan, Woolf 2002, Fontán-Lozano, Romero-Granados et al. 2009). In the cytoplasm, DREAM interacts with the carboxy-terminal fragment (CTF) of presenilin-1(PS1) and facilitate the production of the A $\beta 42$ peptide, a peptide involved in Alzheimer's disease (Buxbaum, Choi et al. 1998). DREAM also binds to the T1 domain of potassium channels and regulates their translocation to membrane and gating kinetics (An, Bowlby et al. 2000). The role of DREAM in memory retention, learning, pain sensing, and Alzheimer's disease emphasizes the multifunctional nature of this protein (Buxbaum, Choi et al. 1998, Costigan, Woolf 2002, Fontán-Lozano, Romero-Granados et al. 2009). In the absence of interacting partners, DREAM does not possess an enzymatic activity, and thus its ability to regulate diverse biological processes arises from interaction with different binding partners.

The three-dimensional NMR structure of $\mathrm{Ca}^{2+}$-bound DREAM (residue 78-256) is presented in Figure 3.1 (Lusin, Vanarotti et al. 2008). DREAM contains two domains, and each of the domains is composed of two EF-hands (Figure 3.1). A short N- terminus $\alpha$-helix together with EF1 (residue 90-119) and EF2 (residue 128-157) form an N-terminal 
domain, whereas EF3 (residue 163-192) and EF4 (residue 211-240) and a 13 residue long C-terminal $\alpha$-helix form a $\mathrm{C}$-terminal domain. Both domains are arranged in a tandem array and form a flexible structure with the solvent-exposed hydrophobic groove (Lusin, Vanarotti et al. 2008). The four EF-hands have the distinct metal binding property (Gifford, Walsh et al. 2007). EF-hand 1 does not bind metal because sterically hindered amino acid residues in the EF-hand loop (Cys 104 and Pro 105) prevent binding of metal; EF-2 binds $\mathrm{Mg}^{2+}$ selectively due to the presence of Asp in the $12^{\text {th }}$ position of the EF-loop instead of usual glutamate; and EF-hand 3 and EF-hand 4 bind $\mathrm{Ca}^{2+}$ selectively (Gifford, Walsh et al. 2007, Lusin, Vanarotti et al. 2008, Osawa, Dace et al. 2005, Osawa, Tong et al. 2001). In addition, we have recently shown that EF-hands in DREAM are capable of binding nonphysiological metal $\mathrm{Tb}^{3+}$ with higher affinity than $\mathrm{Ca}^{2+}$ (Gonzalez, Ramos et al. 2016).

DREAM protein is expressed in the hippocampus, the part of the brain involved in learning and memory consolidation (Carrion, Link et al. 1999, Fontán-Lozano, RomeroGranados et al. 2009). The hippocampus region is strongly impacted by $\mathrm{Pb}^{2+}$ as the $\mathrm{Pb}^{2+}$ exposure alters the expression of 167 hippocampus genes in rats (Schneider, Anderson et al. 2011) and chronic exposure to $\mathrm{Pb}^{2+}$ leads to cell death (Weisskopf, Hu et al. 2004, Chao, Moss et al. 2007). Also, $\mathrm{Pb}^{2+}$ poisoning leads to a decrease in neuronal density in the hippocampus (Weisskopf, Hu et al. 2004). Because DREAM is expressed in the hippocampus and $\mathrm{Pb}^{2+}$ strongly binds to EF-hands in calmodulin, we hypothesize that DREAM may represent a molecular target for $\mathrm{Pb}^{2+}$ poisoning. In this report, we implement a combination of fluorescence and calorimetric techniques to show that $\mathrm{Pb}^{2+}$ binds at the EF-hands in the C-terminal domain of DREAM. Then, we investigate whether $\mathrm{Pb}^{2+}$ binding to DREAM modulates the interaction between DREAM and intracellular partners. We 
report that $\mathrm{Pb}^{2+}$ binds to two EF-hands in DREAM with equilibrium affinity constant higher than that determined for $\mathrm{Ca}^{2+} . \mathrm{Pb}^{2+}$ association triggers changes in the DREAM

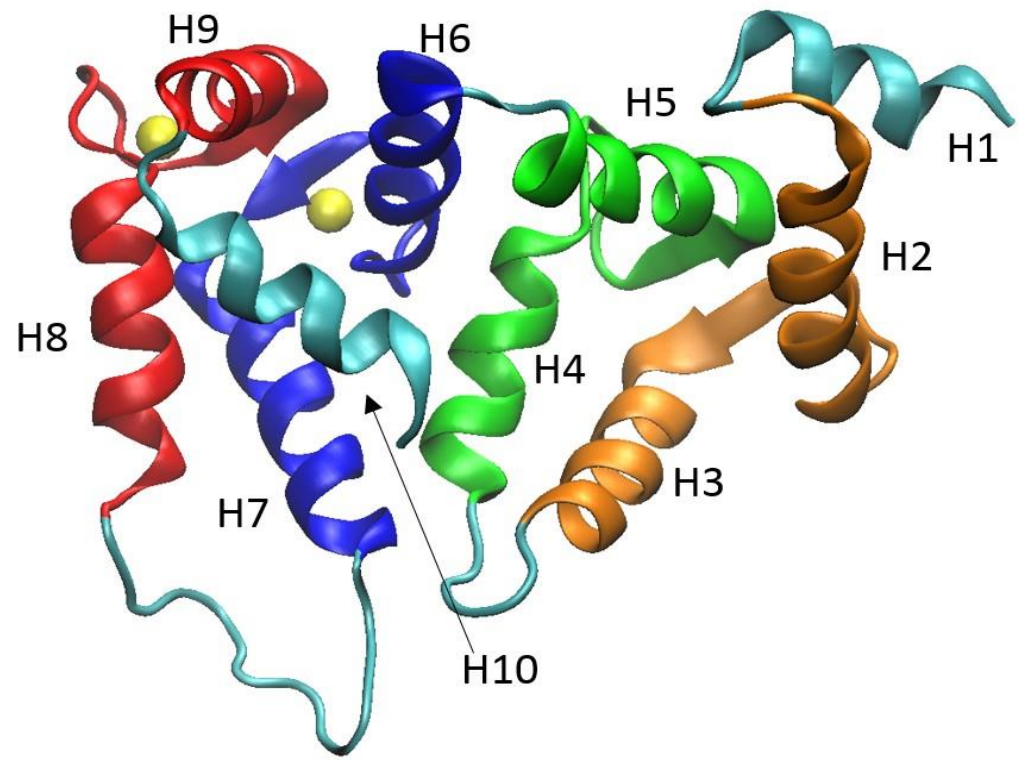

Figure 3.1 Structure of $\mathrm{Ca}^{2+}$ bound DREAM (PDB entry 2JUL). The four EF-hands of DREAM are colored in orange (EF-hand 1), green (EF-hand 2), blue (EF-hand 3), and red (EF-hand 4). The helix-1 and helix-10 are shown in cyan. $\mathrm{Ca}^{2+}$ ions bound to EF-hand 3 and EF-hand 4 are shown as yellow spheres.

secondary and tertiary structures that are analogous to the $\mathrm{Ca}^{2+}$ association, including increased exposure of hydrophobic cavities on the DREAM surface. Such structural reorganization results in increased affinity for small hydrophobic molecules such as 1,8 ANS. $\mathrm{Pb}^{2+}$ association also modulates interactions between DREAM and model peptides that correspond to binding sites of intracellular partners $\mathrm{Kv}$ channels and presenilin. These results indicate that DREAM, and other NCS, may represent molecular targets for $\mathrm{Pb}^{2+}$, and association of $\mathrm{Pb}^{2+}$ to the members of the NCS subfamily may lead to neuropathological conditions attributed to $\mathrm{Pb}^{2+}$ neurotoxicity. 


\subsection{Results and discussion}

3.2.1 $\mathrm{Pb}^{2+}$ Association Alters the Secondary and Tertiary Structure of DREAM.

Tryptophan emission is a popular tool to probe structural transitions in proteins. $\mathrm{Ca}^{2+}$ binding to DREAM protein that lacks first 64 amino acid residues (DREAM $(\Delta 64)$ ) triggers structural changes that lead to a decrease in the emission intensity of the tryptophan residue W169, which is located between the N- and C- terminal domain (Pham, Dhulipala et al. 2015, Carrion, Mellstrom et al. 1998). The emission spectra of DREAM( $\Delta 64)$ in the presence of $\mathrm{Ca}^{2+}, \mathrm{Pb}^{2+}, \mathrm{Ca}^{2+} \mathrm{Pb}^{2+}$ and in the apoform are shown in Figure 3.2A. The emission intensity of $\operatorname{Trp}$ decreases by $\sim 24 \%$ at $335 \mathrm{~nm}$ upon binding of $\mathrm{Pb}^{2+}$ to the apoprotein, pointing towards analogous structural rearrangement at the interface between EF-hand 2 and EF-hand 3 as observed upon $\mathrm{Ca}^{2+}$ binding. Interestingly, the quenching of Trp emission intensity in $\mathrm{Pb}^{2+}$-bound $\operatorname{DREAM}(\Delta 64)$ is more pronounced compared with $\mathrm{Ca}^{2+}$-bound DREAM( $(\Delta 64)$. Addition of $\mathrm{Pb}^{2+}$ to $\mathrm{Ca}^{2+}$ bound DREAM( $\left.\Delta 64\right)$ results in the emission spectrum that is nearly superimposable with the emission spectrum of $\mathrm{Pb}^{2+}$-bound $\operatorname{DREAM}(\Delta 64)$, suggesting that $\mathrm{Pb}^{2+}$ effectively displaces $\mathrm{Ca}^{2+}$ from the EF-hands, pointing towards a higher affinity of $\mathrm{Pb}^{2+}$ for the $\mathrm{Ca}^{2+}$ binding sites in DREAM. To determine the equilibrium dissociation constant for $\mathrm{Pb}^{2+}$ interactions with DREAM, $\mathrm{Pb}^{2+}$

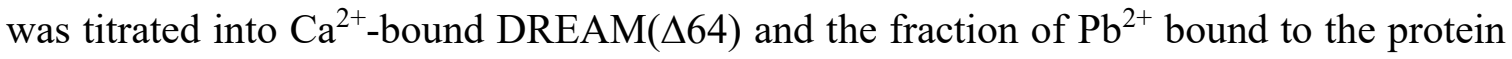
was calculated from the decrease in the Trp emission intensity at $329 \mathrm{~nm}$ according to Equation 1 (Figure 3.2B). Data were fitted to Equation 2 and the apparent dissociation constant was determined to be $2.0 \pm 0.2 \mu \mathrm{M}$ (Figure 3.2B). Using the $\mathrm{K}_{\mathrm{d}}$ value previously 

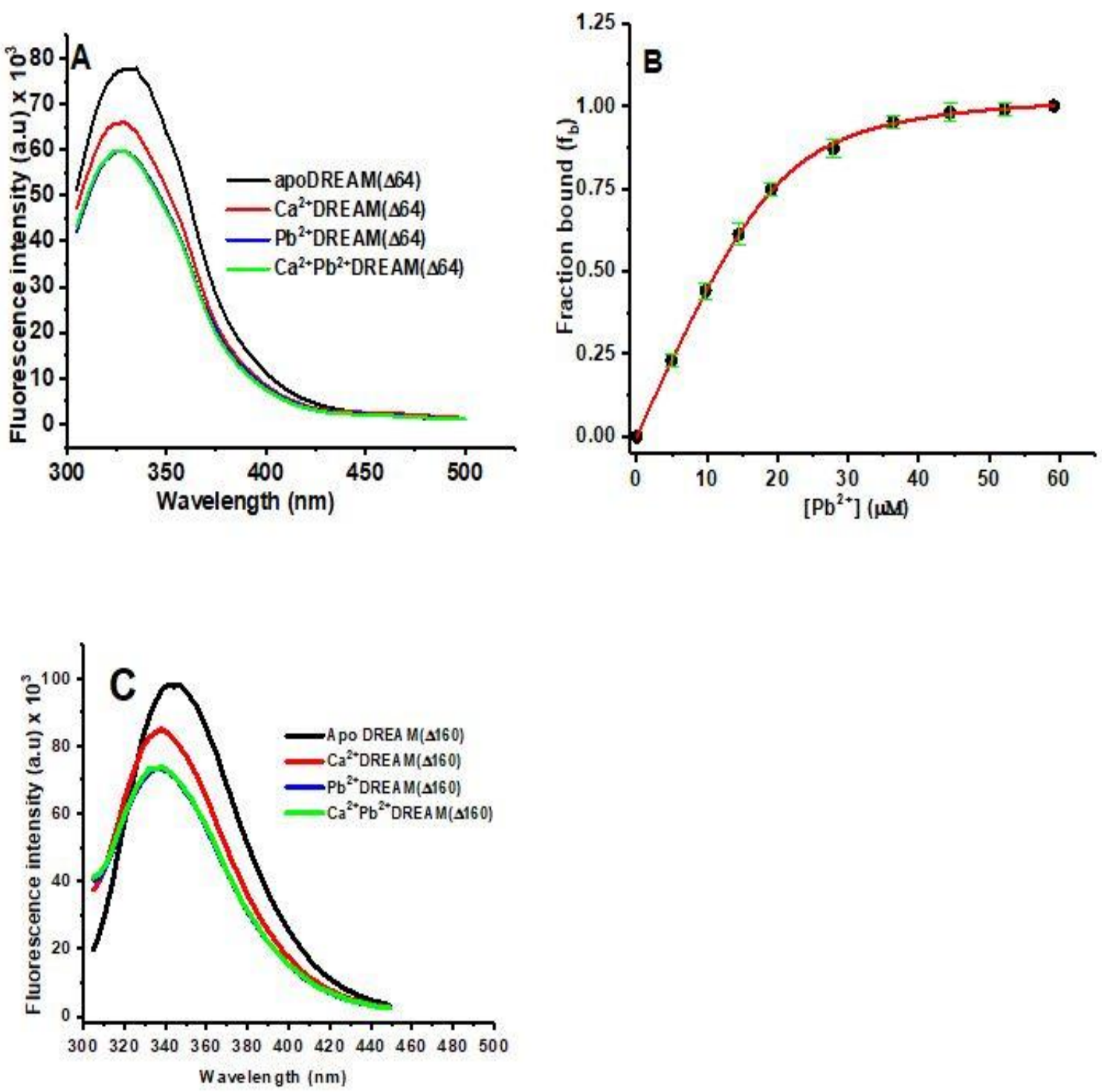

Figure 3.2. Fluorescence emission spectra of $20 \mu \mathrm{M}$ DREAM $(\Delta 64)$ in the metal free form and in the presence of $\mathrm{Ca}^{2+}$ and/or $\mathrm{Pb}^{2+}$. Conditions: $20 \mathrm{mM}$ Tris buffer (pH 7.4), $1 \mathrm{mM}$ EDTA, $1 \mathrm{mM} \mathrm{Ca}^{2+}, 40 \mu \mathrm{M} \mathrm{Pb}^{2+}$, and $1 \mathrm{mM} \mathrm{Ca}^{2+}$ and $40 \mu \mathrm{M} \mathrm{Pb}^{2+}$; samples were excited at $295 \mathrm{~nm}$ and experiments were carried out at $20{ }^{\circ} \mathrm{C} \mathrm{(A)}$. Titration curve for $\mathrm{Pb}^{2+}$ binding to $\mathrm{Ca}^{2+} \mathrm{DREAM}$. The fraction bound was determined based on decrease in Trp169 emission intensity at increasing $\mathrm{Pb}^{2+}$ concentration. Conditions: $20 \mu \mathrm{M}$ DREAM $(\Delta 64)$ in $5 \mathrm{mM}$ Tris, $\mathrm{pH} 7.4$ and 100 $\mu \mathrm{M} \mathrm{Ca}^{2+}$. Experimental points correspond to the averadge value determined from three independent measurments. The solid line represents the fit using Equation 2; the standard deviations are shown as error bars (B). Fluorescence emission spectra of $20 \mu \mathrm{M}$ DREAM $(\Delta 160)$. Conditions as described for Figure 3.2A (C). 
determined for $\mathrm{Ca}^{2+}$ binding to apoDREAM $(\Delta 64)\left(\mathrm{K}_{\mathrm{d}}=1 \mu \mathrm{M}\right)$, (Osawa, Dace et al. 2005) the equilibrium dissociation constant for the $\mathrm{Pb}^{2+}$ association to $\operatorname{DREAM}(\Delta 64)$ was found to be $20 \pm 2 \mathrm{nM}$ (Equation 3), indicating that $\mathrm{Pb}^{2+}$ binds to EF-hands in DREAM with 50 times higher affinity than $\mathrm{Ca}^{2+}$. This is consistent with the previous study for $\mathrm{Pb}^{2+}$ titration to $\mathrm{CaM}$, in which a 10 -times higher affinity for $\mathrm{Pb}^{2+}$ binding to the $\mathrm{N}$-terminal domain of CaM compared with $\mathrm{Ca}^{2+}$ was reported (Kirberger, Wong et al. 2013).

Among four EF-hands found in the DREAM structure, only EF-hand 3 and EFhand 4 in the $\mathrm{C}$ - terminal domain bind $\mathrm{Ca}^{2+}$ with the high affinity. To probe that $\mathrm{Pb}^{2+}$ replaces $\mathrm{Ca}^{2+}$ from the EF-hand located in the C-terminal domain, we measured the emission spectra of DREAM construct that corresponds to the C-terminal domain, $\operatorname{DREAM}(\Delta 160)$, under conditions identical to those for DREAM $(\Delta 64)$ (Figure $3.2 \mathrm{C}) . \mathrm{Pb}^{2+}$ association to $\operatorname{DREAM}(\Delta 160)$ shows an analogous decrease in emission intensity as observed for $\mathrm{Ca}^{2+}$ binding in agreement with $\mathrm{Pb}^{2+}$ binding to EF-3, EF-4, or both. The Trp emission spectra are slightly red-shifted in $\operatorname{DREAM}(\Delta 160)\left(\lambda_{\max }\right.$ for apo and $\mathrm{Pb}^{2+}$ $\operatorname{DREAM}((\Delta 160)$ are $341 \mathrm{~nm}$ and $335 \mathrm{~nm}$, respectively $)$ compared with DREAM $\left(\Delta 64\left(\lambda_{\max }\right.\right.$ for apo and $\mathrm{Pb}^{2+} \operatorname{DREAM}((\Delta 64)$ are $335 \mathrm{~nm}$ and $329 \mathrm{~nm}$, respectively $\left.)\right)$, which could be explained by an increased solvent exposure of Trp side chain in this construct. $\mathrm{Pb}^{2+}$ association to DREAM $(\Delta 64)$ is further supported by CD data. Far-UV CD data reveals that $\mathrm{CD}$ signal at 208-222 $\mathrm{nm}$ decreases upon $\mathrm{Pb}^{2+}$ binding (Figure 3.3A). Interestingly, the $\mathrm{CD}$ spectrum of $\mathrm{Pb}^{2+}$-bound $\operatorname{DREAM}(\Delta 64)$ is intermediate between the signal for apo and $\mathrm{Ca}^{2+} \operatorname{DREAM}(\Delta 64)$. The decrease in CD signal in $\mathrm{Ca}^{2+}$ - or $\mathrm{Pb}^{2+}$-bound DREAM $(\Delta 64)$ can be attributed to an increase of $\alpha$-helical content as well as rearrangement of $\alpha$-helices. The 
addition of $\mathrm{Pb}^{2+}$ to $\mathrm{Ca}^{2+} \operatorname{DREAM}(\Delta 64)$ leads to a $\mathrm{CD}$ spectrum that is identical with $\mathrm{Pb}^{2+}$ bound $\operatorname{DREAM}(\Delta 64)$, providing further evidence of $\mathrm{Ca}^{2+}$ displacement by $\mathrm{Pb}^{2+}$. Analysis of the far-UV CD data using secondary structure prediction tool K2D3 shows that $\alpha$-helical property of the protein increases from $64 \%$ in apoDREAM( $(\Delta 64)$ to $72 \%$ in $\mathrm{Pb}^{2+}$ $\operatorname{DREAM}(\Delta 64)$ and $\mathrm{Ca}^{2+} \mathrm{Pb}^{2+} \operatorname{DREAM}(\Delta 64)$ and $74 \%$ in $\mathrm{Ca}^{2+} \operatorname{DREAM}(\Delta 64)$. Contrary to far-UV CD data, near-UV CD signal increases upon $\mathrm{Ca}^{2+}$ and $\mathrm{Pb}^{2+}$ binding (Figure 3.3B).
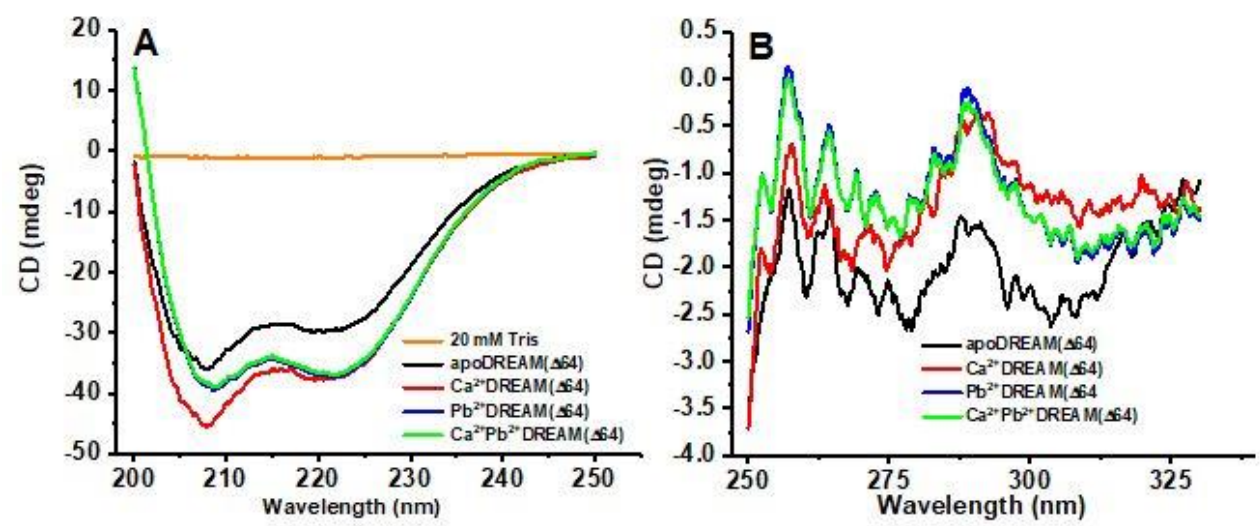

Figure 3.3. (A) Far-UV CD spectra of $20 \mu \mathrm{M}$ DREAM $(\Delta 64)$ in the presence of $1 \mathrm{mM}$ EDTA, $1 \mathrm{mM} \mathrm{Ca}^{2+}, 40 \mu \mathrm{M} \mathrm{Pb}^{2+}$, and $\left(1 \mathrm{mM} \mathrm{Ca}^{2+}\right.$ and $\left.40 \mu \mathrm{M} \mathrm{Pb}^{2+}\right)$ in $20 \mathrm{mM}$ Tris (pH 7.4). (B) Near-UV CD spectra of $40 \mu \mathrm{M} \operatorname{DREAM}(\Delta 64)$ in the presence of $1 \mathrm{mM}$ EDTA, $1 \mathrm{mM} \mathrm{Ca}{ }^{2+}, 40 \mu \mathrm{M} \mathrm{Pb}^{2+}$, and $\left(1 \mathrm{mM} \mathrm{Ca}^{2+}\right.$ and 40 $\left.\mu \mathrm{M} \mathrm{Pb}^{2+}\right)$ in $5 \mathrm{mM}$ phosphate (pH 7.4).

The peaks between 256-272 nm could be attributed to phenylalanine residues, whereas the peak near $292 \mathrm{~nm}$ is likely due to the tyrosine residue. The comparable increase in the nearUV CD-signal upon $\mathrm{Ca}^{2+}$ and $\mathrm{Pb}^{2+}$ binding suggest that the tertiary structure of $\mathrm{Pb}^{2+}$-bound $\operatorname{DREAM}(\Delta 64)$ is analogous to $\mathrm{Ca}^{2+}$-bound DREAM $(\Delta 64)$. The near-uv CD spectrum of $\mathrm{Pb}^{2+}$-DREAM $(\Delta 64)$ is identical with $\mathrm{Ca}^{2+} \mathrm{Pb}^{2+}$-DREAM $(\Delta 64)$ corroborate far-UV CD data and fluorescence data that $\mathrm{Pb}^{2+}$ displaces $\mathrm{Ca}^{2+}$ from the EF-hand. 
We have also determined the Trp169 lifetime in phase modulation measurements (Figure 3.4 and Table 3.1). The data were analyzed using a sum of a Gaussian distribution model and a discrete exponential decay. Two lifetimes were observed for both apo and metal-bound $\operatorname{DREAM}(\Delta 64)$ and were previously attributed to the presence of two $\operatorname{Trp} 169$ rotamers ( $\mathrm{t}$ and $\mathrm{g}+$ ) (Pham, Dhulipala et al. 2015). G+-rotamer corresponds to more solvent-exposed Trp 169 side chain and thus displays the longer lifetime, whereas being more efficiently quenched by nearby amino acid residues, the t-rotamer exhibits a shorter lifetime (Pham, Dhulipala et al. 2015). Upon binding of $\mathrm{Pb}^{2+}$, the average lifetime of Trp 169 decreases from $4.4 \pm 0.1 \mathrm{~ns}$ to $3.8 \pm 0.1 \mathrm{~ns}$, which is due to a decrease in the fractional intensity contribution associated with the longer lifetime and slightly faster Gaussian decay (from $2.1 \pm 0.1 \mathrm{~ns}$ for apoDREAM to $1.6 \pm 0.2 \mathrm{~ns}$ for $\mathrm{Ca}^{2+}$ or Pb $^{2+}$ DREAM) (Table 3.1). These data are consistent with the steady-state emission data, suggesting that the association of $\mathrm{Pb}^{2+}$ to DREAM triggers a structural transition that is analogous to that triggered by $\mathrm{Ca}^{2+}$ association to EF-hand 3 and EF-hand 4. 


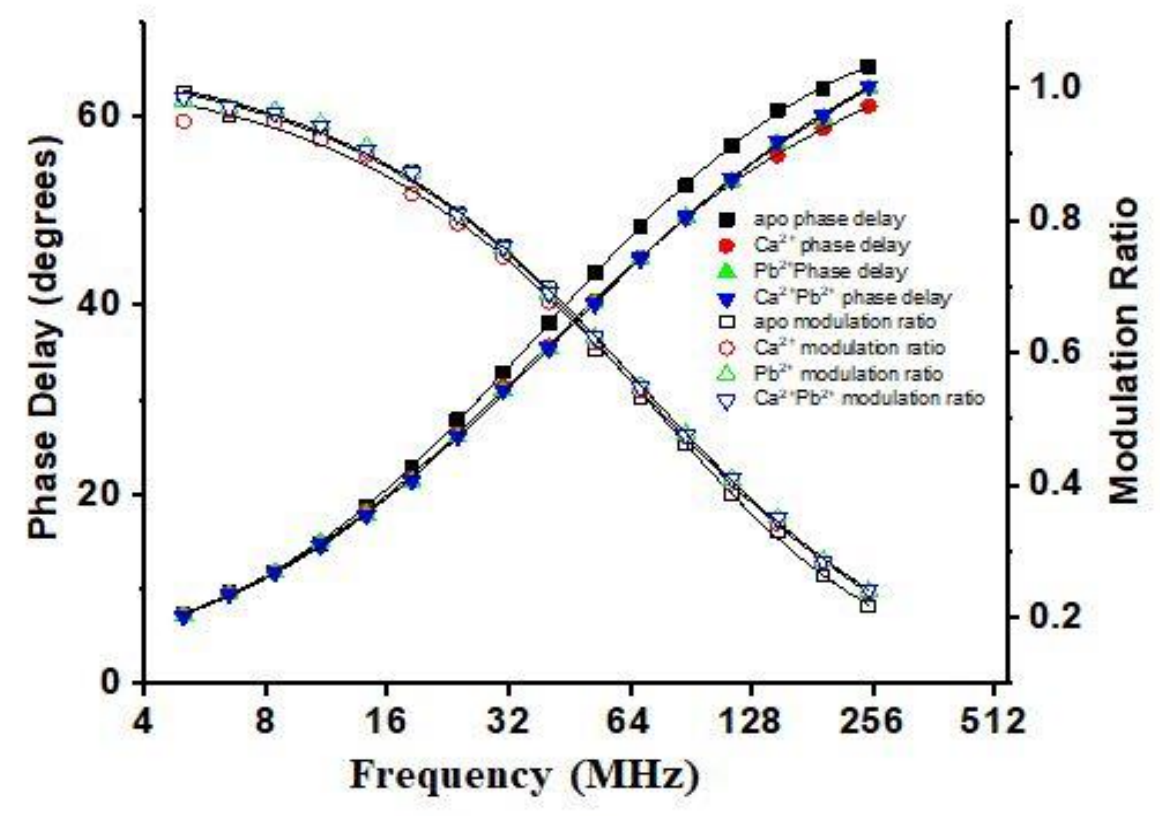

Figure 3.4. Frequency-domain intensity decay of $20 \mu \mathrm{M}$ apo (squares), $\mathrm{Ca}^{2+}$ (circle), $\mathrm{Pb}^{2+}$ (up triangle), and $\mathrm{Ca}^{2+} \mathrm{Pb}^{2+}$ (down triangle)DREAM $(\Delta 64)$ in $20 \mathrm{mM}$ Tris ( $\mathrm{pH}$ 7.4). The phase delay is shown as solid symbols and modulation ratio as empty symbols. Solid lines represent data fitting using a (one Gaussian distribution and single exponential decay) model. 
Table 3.1. Fluorescence decay parameters for $\operatorname{DREAM}(\Delta 64)$ as a function of $\mathrm{Ca}^{2+/} \mathrm{Pb}^{2+}$ binding to EF-hands.

\begin{tabular}{|c|c|c|c|c|c|c|c|c|c|}
\hline Samples & $\tau_{1}(\mathrm{~ns})$ & $\alpha_{1}(\%)$ & $\tau_{2}(\mathrm{~ns})$ & $\alpha_{2}(\%)$ & $\mathrm{f}_{1}(\%)$ & $\mathrm{f}_{2}(\%)$ & $\mathrm{W}_{1}$ & $<\tau>(\mathrm{ns})$ & $\chi^{2}$ \\
\hline apo & $2.1 \pm 0.1$ & $17.1 \pm 0.2$ & $5.9 \pm 0.1$ & $82.9 \pm 0.2$ & $41.2 \pm 0.4$ & $59.8 \pm 0.4$ & $1.1 \pm 0.1$ & $4.4 \pm 0.1$ & 2.9 \\
\hline $\mathrm{Ca}^{2+}$ & $1.6 \pm 0.2$ & $15.9 \pm 0.4$ & $5.9 \pm 0.1$ & $84.1 \pm 0.4$ & $49.3 \pm 0.3$ & $51.7 \pm 0.3$ & $1.0 \pm 0.1$ & $3.8 \pm 0.1$ & 1.6 \\
\hline $\mathrm{Pb}^{2+}$ & $1.6 \pm 0.2$ & $15.8 \pm 0.3$ & $5.9 \pm 0.1$ & $84.2 \pm 0.3$ & $49.6 \pm 0.4$ & $51.4 \pm 0.4$ & $1.0 \pm 0.1$ & $3.8 \pm 0.1$ & 1.6 \\
\hline $\mathrm{Ca}^{2+} \mathrm{Pb}^{2+}$ & $1.6 \pm 0.2$ & $15.8 \pm 0.5$ & $5.9 \pm 0.1$ & $84.2 \pm 0.5$ & $50.5 \pm 0.5$ & $49.5 \pm 0.5$ & $1.0 \pm 0.1$ & $3.8 \pm 0.2$ & 1.6 \\
\hline
\end{tabular}

Recovered from global analysis of the phase and modulation data using a (one Gaussian distribution and discrete single exponential decay) model. $\tau_{1}$ is the decay time of the Gaussian distribution with a width of distribution $w_{1}$; $\tau_{2}$ is the lifetime of the discrete single exponential term; $\alpha_{1}$ and $\alpha_{2}$ are pre-exponential decays; $f_{1}$ and $f_{2}$ are exponential decay fraction. $\chi^{2}$ represents the goodness of the fit. Lifetime parameters represent average of three independent measurements; standard deviations are shown as errors. Phase and modulation errors were set at $\leq 0.2^{\circ}$ and $\leq 0.004$ respectively. 
3.2.2 $\mathrm{Pb}^{2+}$ Binding promotes DREAM Interactions with Hydrophobic Molecule 1,8-ANS.

We have previously shown that $\mathrm{Ca}^{2+}$ modulates DREAM affinity for small organic molecules such as 1,8-ANS and arachidonic acid (Gonzalez, Miksovska 2014). To investigate whether $\mathrm{Pb}^{2+}$ binding influences DREAM interactions with hydrophobic molecules, we have monitored $\mathrm{Pb}^{2+}$-bound DREAM interactions with 1,8-ANS. This probe offers a spectroscopic approach for assessing the hydrophobicity of the protein surface due to its increase in the emission intensity upon the association to hydrophobic patches on the protein surface. 1,8-ANS emission intensity increases in the presence of apoDREAM $(\Delta 64)$ because of 1,8 -ANS binding to the protein. The further increase in the emission of $\mathrm{Pb}^{2+}$ or $\mathrm{Ca}^{2+}$-bound DREAM $(\Delta 64)$ compared with the apo form suggest that 1,8-ANS associates to the hydrophobic cavities on the DREAM $(\Delta 64)$ surface with a higher affinity in the presence of $\mathrm{Ca}^{2+}$ or $\mathrm{Pb}^{2+}$ (Figure 3.5A). The emission spectrum of $\mathrm{Pb}^{2+}$-bound $\operatorname{DREAM}(\Delta 64): 1,8$-ANS complex is identical with $\mathrm{Ca}^{2+} \mathrm{Pb}^{2+}$-DREAM $(\Delta 64)$, suggesting that $\mathrm{Pb}^{2+}$ binds to $\operatorname{DREAM}(\Delta 64)$ with higher affinity and displaces $\mathrm{Ca}^{2+}$ from EF-hand, which is consistent with CD and Trp emission data. DREAM(160):1,8-ANS complexes show emission spectra that are analogous to $\operatorname{DREAM}(\Delta 64): 1,8$-ANS complexes (Figure 3.5B). We determined the affinity of the 1,8-ANS probe for DREAM by titrating the constant amount of 1,8-ANS against $\operatorname{DREAM}(\Delta 64)$ (Figure 3.5C). The fraction of 1,8ANS bound to protein was calculated from the increase in emission intensity at $466 \mathrm{~nm}$ (Equation 1), and binding isotherm (Figure 3.5C ) was analyzed according to Equation 2. The $\mathrm{K}_{\mathrm{d}}$ value obtained for 1,8 -ANS binding to $\mathrm{Pb}^{2+}$-bound $\operatorname{DREAM}(\Delta 64)$ is $87 \pm 4 \mu \mathrm{M}$ (Figure 3.5C ), which is similar to the $\mathrm{K}_{\mathrm{d}}$ value obtained for $\mathrm{Ca}^{2+}$-bound $\operatorname{DREAM}(\Delta 64)$ $(107 \pm 11 \mu \mathrm{M})\left(\right.$ data not shown), suggesting that $\mathrm{Ca}^{2+}$ - and $\mathrm{Pb}^{2+}$-bound $\operatorname{DREAM}(\Delta 64)$ have 
similar affinity for 1,8 -ANS. The affinity of $\mathrm{Pb}^{2+} \operatorname{DREAM}(\Delta 64)$ for 1,8 -ANS complex is approximately two times higher compared with the value previously determined for the apo form $(195 \pm 20 \mu \mathrm{M})$ (Gonzalez, Miksovska 2014), which is consistent with an increased accessibility of the 1,8-ANS binding sites in $\mathrm{Pb}^{2+}$ bound protein.
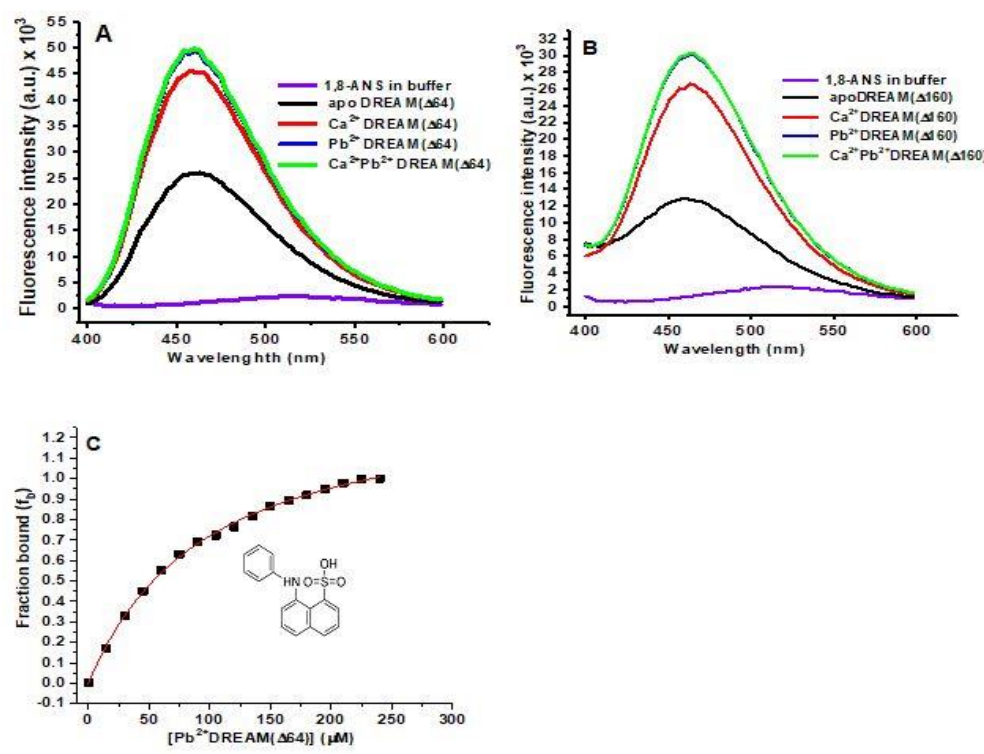

Figure 3.5. Emission spectra of (A) 1,8-ANS:DREAM $(\Delta 64)$ and (B) 1,8-ANS:DREAM $(\Delta 160)$ complexes as a function of $\mathrm{Pb}^{2+}$ or $\mathrm{Ca}^{2+}$ binding to the protein. Conditions: $20 \mu \mathrm{M}$ $\operatorname{DREAM}(\Delta 64)$ and $20 \mu \mathrm{M}$ 1,8-ANS; excited at $350 \mathrm{~nm}$. (C) Titration of $10 \mu \mathrm{M} 1,8$-ANS with $\mathrm{Pb}^{2+} \operatorname{DREAM}(\Delta 64)$. The fit of the experimental data using Equation 2 is shown as a solid line. Inset: 1,8-ANS structure.

The 1,8-ANS fluorescence lifetimes are also sensitive to structural changes associated with $\mathrm{Ca}^{2+}$ binding to $\operatorname{DREAM}(\Delta 64)$. To investigate whether $\mathrm{Pb}^{2+}$ binding to DREAM influences the lifetime of 1,8-ANS, we measured the lifetime of $\operatorname{DREAM}(\Delta 64)$ :1,8-ANS complexes (Figure 3.6). 1,8-ANS bound to apoDREAM exhibits two fluorescent lifetimes ( $\tau_{1}=4.8 \pm 0.1 \mathrm{~ns}$ and $\left.\tau_{2}=17.2 \pm 0.1 \mathrm{~ns}\right)$, which can be attributed to 1,8 -ANS associated to at least two 1,8-ANS binding sites on DREAM surface (Table 
3.2). Previously, we used AutoDock software version 4.2 to identify 1,8-ANS binding sites on DREAM surface (Gonzalez, Miksovska 2014, Morris, Huey et al. 2009). One binding site was found between the helix 8 and helix 9 (EF-hand 4). This binding site is partially solvent-exposed and 1,8-ANS bound to this side was associated with a shorter lifetime. The second site was identified between the EF-hand 3 and EF-hand 4. This is a more

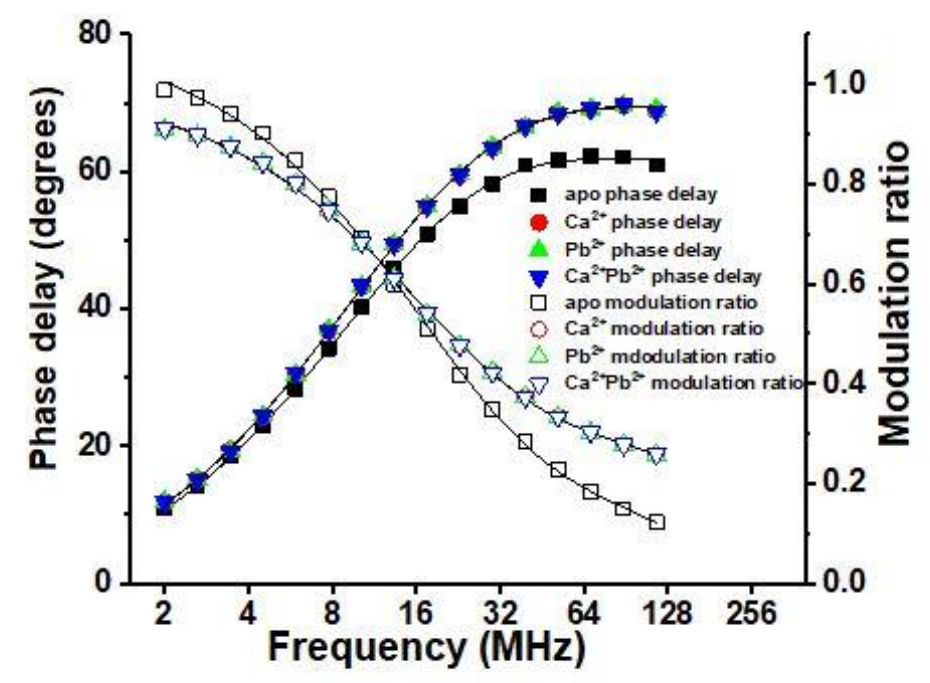

Figure 3.6. Frequency-domain intensity decay of $40 \mu \mathrm{M} 1,8-$ ANS binding to $40 \mu \mathrm{M}$ DREAM $(\Delta 64)$ in presence of EDTA (squares), $\mathrm{Ca}^{2+}$ (circle), $\mathrm{Pb}^{2+}$ (up triangle), and $\mathrm{Ca}^{2+} \mathrm{Pb}^{2+}$ (down triangle). The phase delay is shown as solid symbols and modulation ratio as empty symbols. Solid lines represent data fitting using three-exponential decay model.

hydrophobic side and 1,8-ANS bound to this site is likely to exhibit a longer lifetime. Addition of $\mathrm{Pb}^{2+}$ to apoDREAM( $(\Delta 64)$ :1,8-ANS complex leads to an increase in the longer lifetime ( $\left.\tau_{2}=18.4 \pm 0.2 \mathrm{~ns}\right)$ and an approximately two-fold increase in the corresponding pre-exponential parameter $\alpha_{2}$ compared with the apoprotein, suggesting $\mathrm{Pb}^{2+}$ association to EF-hand 3 and EF-hand 4 increases the affinity for 1,8-ANS binding to the DREAM and 
leads to an increased accessibility of the hydrophobic cavity in the C-terminal domain. Individual 1,8-ANS lifetimes are moderatedly changed by $\mathrm{Pb}^{2}$ to DREAM $(\Delta 64)$, which is consistent with 1,8-ANS binding to identical binding sites in $\mathrm{Ca}^{2+}$-free, $\mathrm{Pb}^{2+}$-, and $\mathrm{Ca}^{2+}$ bound protein. 
Table 3.2. Fluorescence decay parameters for DREAM $(\Delta 64)-1,8$-ANS complexes as a function of $\mathrm{Ca}^{2+}$ and/or $\mathrm{Pb}^{2+}$ binding to EF-hands.

\begin{tabular}{|c|c|c|c|c|c|c|}
\hline 1,8 -ANS & $\tau_{1}(\mathrm{~ns})$ & $\alpha_{1}(\%)$ & $\tau_{2}(\mathrm{~ns})$ & $\alpha_{2}(\%)$ & $\mathrm{f}_{2}(\%)$ & $\chi^{2}$ \\
\hline $\operatorname{apoDREAM}(\Delta 64)$ & $4.8 \pm 0.1$ & $11.3 \pm 0.8$ & $17.2 \pm 0.1$ & $16.2 \pm 0.714 .3 \pm 0.5$ & $50.9 \pm 0.6$ & 2.0 \\
\hline $\mathrm{Ca}^{2+} \mathrm{DREAM}(\Delta 64)$ & $5.1 \pm 0.2$ & $15.1 \pm 0.7$ & $18.3 \pm 0.2$ & $34.5 \pm 0.58 .9 \pm 0.4$ & $88.5 \pm 0.8$ & 1.8 \\
\hline $\mathrm{Pb}^{2+} \operatorname{DREAM}(\Delta 64)$ & $5.1 \pm 0.3$ & $15.1 \pm 0.6$ & $18.4 \pm 0.2$ & $33.2 \pm 0.49 .1 \pm 0.3$ & $88.3 \pm 0.6$ & 1.7 \\
\hline $\begin{array}{l}\mathrm{Pb}^{2+} \mathrm{Ca}^{2+} \mathrm{DREAM}(\Delta 6 \\
\text { 4) }\end{array}$ & $5.0 \pm 0.2$ & $15.2 \pm 0.5$ & $18.4 \pm 0.3$ & $35.3 \pm 0.69 .3 \pm 0.4$ & $88.1 \pm 0.5$ & 1.1 \\
\hline \multicolumn{7}{|c|}{$\begin{array}{l}\text { Data recovered from Globals software using a sum of three discrete exponential decays with fixed } 1,8 \text { - } \\
\text { ANS lifetime }\left(\tau_{0}=0.28 \mathrm{~ns}\right) \text {. Lifetime parameters represent average of three independent } \\
\text { neasurements; standard deviations are shown as errors. Phase and modulation errors were set at } \leq 0.2^{\circ} \\
\text { nd } \leq 0.004 \text { respectively. }\end{array}$} \\
\hline
\end{tabular}




\subsection{3 $\mathrm{Pb}^{2+}$ Binding to DREAM is Entropy Driven.}

To complement the results from fluorescence experiments, ITC studies were carried out to determine thermodynamic parameters for the $\mathrm{Pb}^{2+}$ association to $\mathrm{Ca}^{2+} \operatorname{DREAM}(\Delta 64)$ and $\mathrm{Ca}^{2+} \operatorname{DREAM}(\Delta 160)$ (Figure 3.7A,B). Isothermal calorimetry data
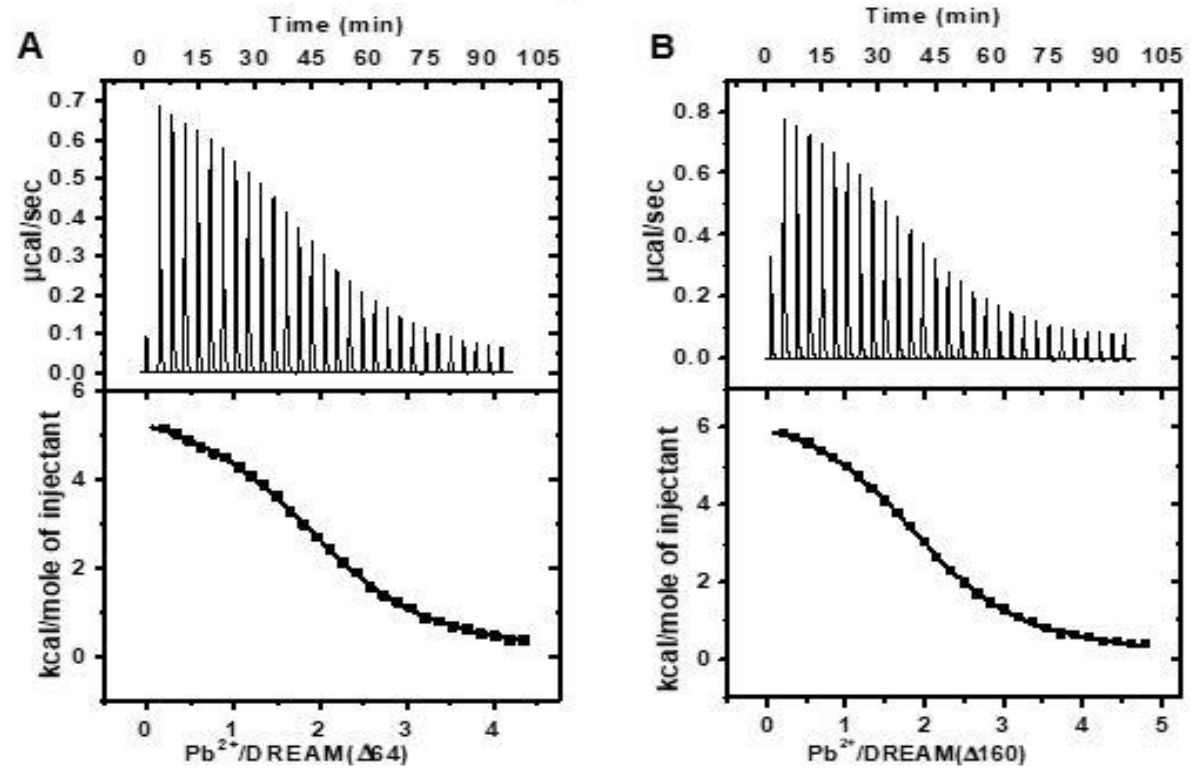

Figure 3.7. ITC isotherms for $\mathrm{Pb}^{2+}$ displacement of $\mathrm{Ca}^{2+}$ from (A) DREAM $(\Delta 64)$ and $(B)$ DREAM $(\Delta 160)$. The upper panels of the profile reflect the thermal power expressed in units of microcalories per second, whereas the lower panel represents integrated reaction heats $(\Delta \mathrm{H})$ expressed in units of kilocalories per mole. The solid line represents the best fitting curve with parameters listed in Table 3.3.

reveal that displacement of $\mathrm{Ca}^{2+}$ from DREAM is endothermic and can be modeled as a sequential process with two binding sites. Satisfactory fits were obtained using both a oneset of sites model and sequential process with two sites. Because we used the sequential model previously to analyze $\mathrm{Tb}^{3+}$ binding to $\mathrm{Ca}^{2+}$ DREAM (Gonzalez, Ramos et al. 2016), a sequential model with two sites was employed here and the thermodynamic parameters are listed in Table 3.3. Two $\mathrm{Pb}^{2+}$ ions bind to $\mathrm{Ca}^{2+} \operatorname{DREAM}(\Delta 64)$ with $\mathrm{K}_{\text {app }}$ values $2.63 \pm$ 
$0.18 \mu \mathrm{M}$ and $8.74 \pm 0.02 \mu \mathrm{M}$, respectively. The profile of the ITC isotherm for $\mathrm{Pb}^{2+}$ displacement of $\mathrm{Ca}^{2+}$ bound to $\operatorname{DREAM}(\Delta 64)$ is similar to binding isotherm for $\mathrm{Tb}^{3+}$ displacement of $\mathrm{Ca}^{2+}$ bound to $\operatorname{DREAM}(\Delta 64)$ (Gonzalez, Ramos et al. 2016), points towards a similar mechanism for $\mathrm{Ca}^{2+}$ displacement from $\operatorname{DREAM}(\Delta 64)$ by $\mathrm{Pb}^{2+}$ and $\mathrm{Tb}^{3+}$ (Table 3.3). The calorimetric study for the $\mathrm{Pb}^{2+}$ displacement of $\mathrm{Ca}^{2+}$ bound at the EFhands of DREAM $(\Delta 160)$ under conditions identical to those for the $\mathrm{Pb}^{2+}$ displacement of $\mathrm{Ca}^{2+}$ bound at the EF-hands of $\operatorname{DREAM}(\Delta 64)$ (Figure 3.7B) provides thermodynamic parameters that are nearly identical to the parameters for $\mathrm{Pb}^{2+}$ displacement of $\mathrm{Ca}^{2+}$ bound from DREAM $(\Delta 64)$ (Table 3.3). These results suggest that the first 160 amino acid residues have a negligible role in modulating $\mathrm{Pb}^{2+}$ interactions with EF-hands in the DREAM C-terminal domain in the presence of $\mathrm{Ca}^{2+}$. In both protein constructs, $\mathrm{Pb}^{2+}$ associates to two binding sites with a relatively high affinity (see below). However, we cannot eliminate the potential presence of additional sites for $\mathrm{Pb}^{2+}$ of lower affinity in the DREAM N- or C- terminal domain as precipitation of $\mathrm{Ca}^{2+}$-bound DREAM at increased $\mathrm{Pb}^{2+}$ concentrations (above $50 \mu \mathrm{M}$ ) prevented detection of those sites. $\mathrm{Pb}^{2+}$ displacement of $\mathrm{Ca}^{2+}$ from the EF-hand of the $\mathrm{DREAM}(\Delta 64)$ is associated with $\Delta \mathrm{G}$ of $-14.48 \pm 0.08 \mathrm{kcal} \mathrm{mol}^{-1}$, which is identical to the $\mathrm{Pb}^{2+}$ displacement of the $\mathrm{Ca}^{2+}$ from the EF-hand of the $\operatorname{DREAM}(\Delta 160) \Delta \mathrm{G}=-14.52 \pm 0.05 \mathrm{kcal} \cdot \mathrm{mol}^{-1}$. Using the overall $\mathrm{K}_{\text {app }}$ value obtained from ITC measurements $(4.81 \pm 0.06 \mu \mathrm{M})$, we determined the $\mathrm{K}_{\mathrm{d}}$ value for $\mathrm{Pb}^{2+}$ binding to apoDREAM to be $47 \pm 0.6 \mathrm{nM}$, which is similar to the value obtained from fluorescence titration data $\left(K_{d}=20 \pm 2 n M\right)$. The reaction is entropy-driven as positive reaction enthalpy and positive entropy change are associated with the $\mathrm{Pb}^{2+}$ binding to $\mathrm{Ca}^{2+}$ DREAM. The entropy gain cannot be attributed to release of the water molecule(s) to the bulk solvent as the hydration 
sphere of $\mathrm{Ca}^{2+}$ consists of eight water molecules (Jalilehvand, Spångberg et al. 2001), whereas six molecules were found the first hydration sphere of $\mathrm{Pb}^{2+}$ (Marcus 1991) Kirberger and Yang (Kirberger, Yang 2008) have analyzed the X-ray structures of $\mathrm{Ca}^{2+}$-bound $\mathrm{CaM}$ and $\mathrm{Pb}^{2+}$-bound $\mathrm{CaM}$ and reported a marginal change in the EF-hand geometry, suggesting that observed entropy-driven for $\mathrm{Pb}^{2+}$ binding to $\mathrm{Ca}^{2+}$ DREAM may originate from subtle changes in the DREAM conformation. This is in agreement with the spectroscopic data presented here which shows decreased Trp emission intensity in $\mathrm{Pb}^{2+} \mathrm{DREAM}$ compared with $\mathrm{Ca}^{2+}$ DREAM. 
Table 3.3. ITC parameters recovered for $\mathrm{Tb}^{3+}$ and $\mathrm{Pb}^{2+}$ displacement of $\mathrm{Ca}^{2+}$ from EF hands of DREAM using a sequential model.

\begin{tabular}{|c|c|c|c|c|c|c|}
\hline & $\begin{array}{c}\mathrm{K}_{\mathrm{app}_{1}} \\
(\mu \mathrm{M})\end{array}$ & $\begin{array}{l}\mathrm{K}_{\mathrm{app} 2} \\
(\mu \mathrm{M})\end{array}$ & $\begin{array}{c}\Delta \mathrm{H}_{1} \\
\text { (kcal/mol) }\end{array}$ & $\begin{array}{c}\Delta \mathrm{H}_{2} \\
(\mathrm{kcal} / \mathrm{mol})\end{array}$ & $\begin{array}{c}\mathrm{T} \Delta \mathrm{S}_{1} \\
(\mathrm{kcal} / \mathrm{mol})\end{array}$ & $\begin{array}{c}\mathrm{T} \Delta \mathrm{S}_{2} \\
(\mathrm{kcal} / \mathrm{mol})\end{array}$ \\
\hline $\operatorname{DREAM}(\Delta 64)+\mathrm{Pb}^{2+}$ & $2.63 \pm 0.18$ & $8.74 \pm 0.02$ & $6.58 \pm 0.39$ & $6.10 \pm 0.55$ & $14.15 \pm 0.47$ & $13.01 \pm 0.55$ \\
\hline $\operatorname{DREAM}(\Delta 160)+\mathrm{Pb}^{2+}$ & $2.94 \pm 0.41$ & $8.72 \pm 0.01$ & $6.92 \pm 0.07$ & $6.64 \pm 0.09$ & $14.45 \pm 0.03$ & $13.53 \pm 0.08$ \\
\hline $\operatorname{DREAM}(\Delta 64)+\mathrm{Tb}^{3+}$ & 4.5 & 12 & $13 \pm 9$ & $18 \pm 6$ & $20 \pm 9$ & $24 \pm 0.4$ \\
\hline
\end{tabular}


3.2.4 $\mathrm{Pb}^{2+}$ Association to DREAM Facilitates DREAM's Interactions with Intracellular Partners.

Jo et al. (Jo, Jang et al. 2005) previously reported that DREAM binds to the Cterminal of presenilin-1 (PS1-CTF) in the presence of $\mathrm{Ca}^{2+}$ and stimulates $\gamma$ - secretase activity which facilitates the generation of A $\beta 42$ in Alzheimer's disease. To determine whether $\mathrm{Pb}^{2+}$ association to DREAM modulates DREAM affinity for presenilin, the equilibrium binding constant for helix-9 of presenilin-1 (PS1HL9) association to DREAM was measured. We used PS1HL9 as a model for presenilin-1 since pull-down studies have shown that DREAM interacts with residues from helix-9 (Buxbaum, Choi et al. 1998, Pham, Miksovska 2016). FITC-labeled PS1HL9 was titrated with $\mathrm{Pb}^{2+}$-bound $\operatorname{DREAM}(\Delta 64)$ and the change in the anisotropy was probed (Figure 3.8A,B). The fraction
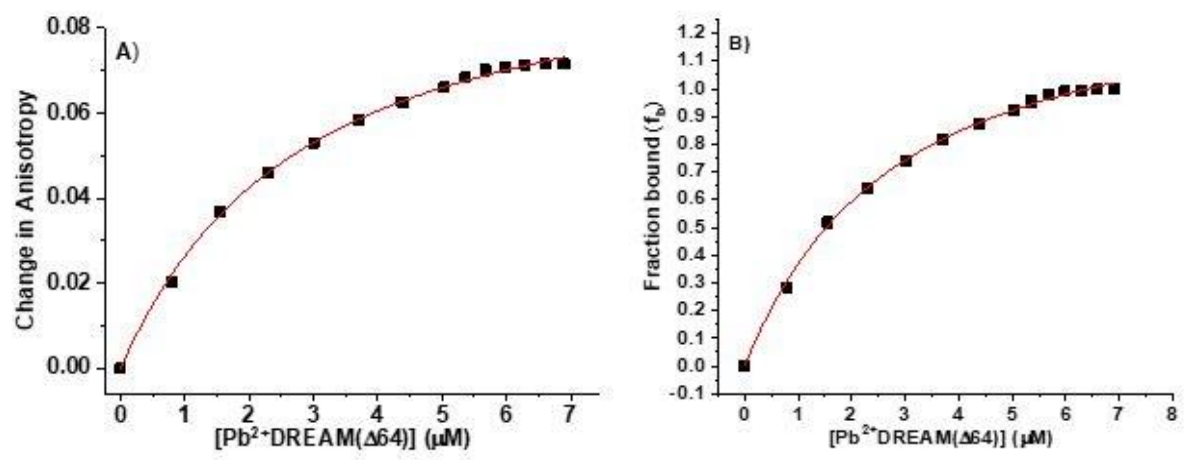

Figure 3.8A,B. Titration of $0.5 \mu \mathrm{M}$ PS1HL9 with $\mathrm{Pb}^{2+} \operatorname{DREAM}(\Delta 64)$. Left panel represents raw anisotropy data. Right panel shows fraction bound that was calculated using Equation 1. In both cases, solid lines correspond to fit of the experimental data using Equation 2.

of DREAM bound to PS1HL9 was then plotted as a function of $\mathrm{Pb}^{2+}$-bound DREAM $(\Delta 64)$ concentration and the dissociation constant was recovered using Equation 2 (Table 3.4 and Figure 3.8A,B). Titration data shows that $\mathrm{Pb}^{2+}$ DREAM binds PS1HL9 with a four-time lower affinity compare to $\mathrm{Ca}^{2+}$-bound $\operatorname{DREAM}(\Delta 64)\left(\mathrm{K}_{\mathrm{d}}=2.56 \pm 0.23 \mu \mathrm{M}\right.$ and $0.6 \pm 0.1$ 
$\mu \mathrm{M}$, respectively). However, the affinity of $\mathrm{Pb}^{2+}$ DREAM and $\mathrm{Ca}^{2+}$ DREAM for PS1HL9 is enhanced compared to apo $\operatorname{DREAM}(\Delta 64)\left(\mathrm{K}_{\mathrm{d}}=183 \pm 12 \mu \mathrm{M}\right)$ (Table 3.4) (Pham, Miksovska 2016). Several studies demonstrated that an early life exposure to $\mathrm{Pb}^{2+}$ promotes developmental reprogramming of APP gene expression, leading to an augmented expression of amyloid precursor protein during adulthood (Huang, Bihaqi et al. 2011, Wu, Basha et al. 2008, Basha, Murali et al. 2005, Basha, Wei et al. 2005). The interaction between $\mathrm{Pb}^{2+}$-bound DREAM and PS1HL9 observed here suggest that elevated concentrations of $\mathrm{Pb}^{2+}$ may directly impact the proteolytic processing of the $\beta$-amyloid precursor protein.

In addition to binding to presenilin, DREAM and other KChIPs are integral auxiliary subunits of A-type potassium voltage channels and the formation of Kv4-KChIP complex has been shown to result in modulation of gating properties, surface expression and subunit assembly of Kv4 channels (An, Bowlby et al. 2000, Gonzalez, Pham et al. 2014, Bahring, Dannenberg et al. 2001, Scannevin, Wang et al. 2004). Although DREAM binds to $\mathrm{Kv}$ channels in a $\mathrm{Ca}^{2+}$-independent manner, the presence of $\mathrm{Ca}^{2+}$ modulates kinetics of Kv channel inactivation (Patel, Campbell et al. 2002). Crystallographic studies demonstrated that the T-1 domain at the N-terminus of $\mathrm{K}_{\mathrm{v}} 4$ interacts with $\mathrm{KChIPs}$ (An, Bowlby et al. 2000, Pioletti, Findeisen et al. 2006, Bahring, Dannenberg et al. 2001, Scannevin, Wang et al. 2004). Two binding sites were found to be involved in binding of $\mathrm{K}_{\mathrm{v}} 4.2$ to $\mathrm{KChIP} 1$, namely site- 1 , which is comprised of non-polar residues $2-22$ and site2, containing more polar residues 70-90 (Callsen, Isbrandt et al. 2005). Our previous research showed that both site-1 and site-2 interact with DREAM in a calcium-dependent manner (Gonzalez, Pham et al. 2014). To determine whether $\mathrm{Pb}^{2+}$ binding to DREAM EF- 
hands modulates interactions between DREAM and site-1 and site-2, we titrated FITCtagged site- 1 and site- 2 with $\mathrm{Pb}^{2+}$-bound $\operatorname{DREAM}(\Delta 64)$ in anisotropy measurements
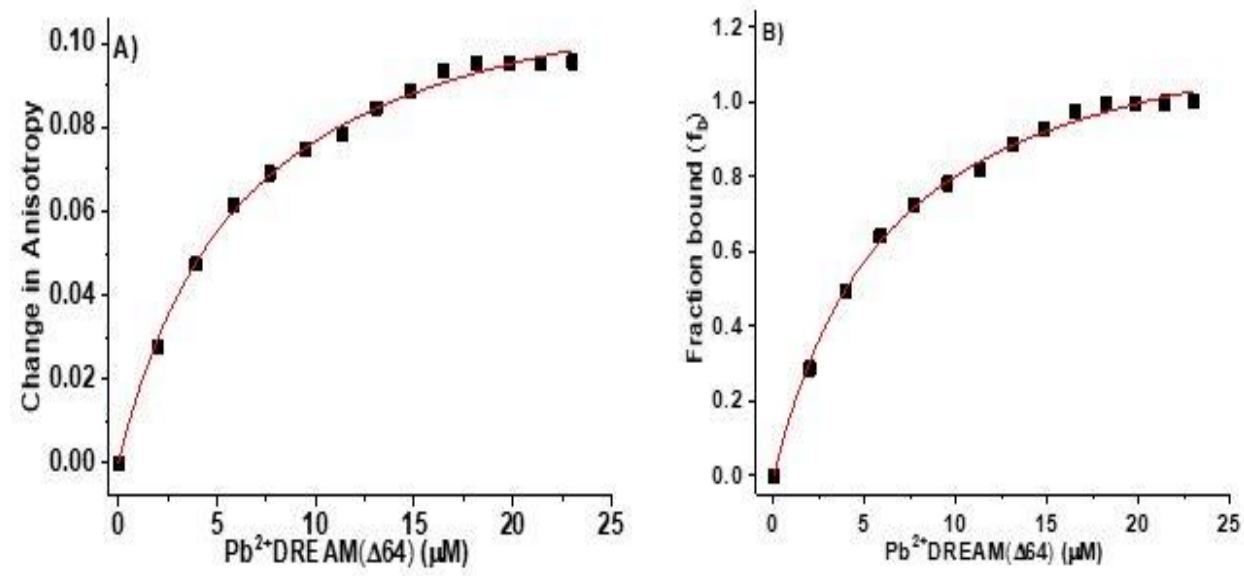

Figure 3.9A,B. Titration of $0.5 \mu \mathrm{M}$ Kv4.3 (2-22) "site-1" with $\mathrm{Pb}^{2+} \operatorname{DREAM}(\Delta 64)$. Right panel shows fraction bound that was calculated using Equation 1. In both cases, solid lines correspond to fit of the experimental data using Equation 2.

(Figure 3.9A,B and 3.10A,B). The equilibrium dissociation constants were determined analogously as described above for PS1HL9 (Table 3.4 and Figure 3.9A,B and 3.10A,B).
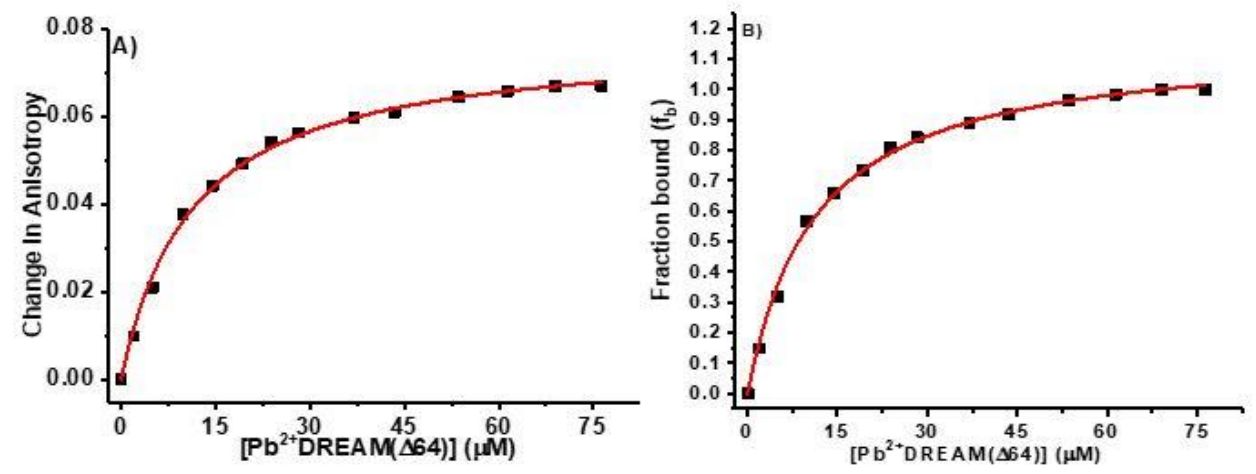

Figure 3.10A,B. Titration of $0.5 \mu \mathrm{M}$ Kv4.3 (70-90) "site-2" with $\mathrm{Pb}^{2+} \operatorname{DREAM}(\Delta 64)$. Right panel shows fraction bound that was calculated using Equation 1. In both cases, solid lines correspond to fit of the experimental data using Equation 2.

The titration data show that $\mathrm{Pb}^{2+}$-bound DREAM has 50-times higher affinity for "site-2"

$\left(\mathrm{K}_{\mathrm{d}}=10.61 \pm 0.40 \mu \mathrm{M}\right)$ and 12-times higher affinity for "site-1" $\left(\mathrm{K}_{\mathrm{d}}=5.9 \pm 0.6 \mu \mathrm{M}\right)$ 
compared with the previously determined dissociation constant for metal-free DREAM (Table 3.4) (Gonzalez, Pham et al. 2014) ${ }^{48}$. Analogous $\mathrm{K}_{d}$ values for $\mathrm{Ca}^{2+}$-bound $\operatorname{DREAM}(\Delta 64)$ and $\mathrm{Pb}^{2+}$-bound $\operatorname{DREAM}(\Delta 64)$ association to site-1 and site-2 indicate that in the absence of $\mathrm{Ca}^{2+}, \mathrm{Pb}^{2+}$ association to DREAM may impact the deactivation kinetics of $\mathrm{Kv}$ channels complexes in a manner analogous to $\mathrm{Ca}^{2+}$ association. Considering the implication of $\mathrm{Kv}$ channels in hippocampus-dependent learning and memory (Lugo, Brewster et al. 2012), the Kv channels:KChIP complexes may represent a molecular target contributing to $\mathrm{Pb}^{2+}$ induced memory and learning deficiencies. Previously, Gorkhali et al. (Gorkhali, Huang et al. 2016) have proposed that neuronal calcium sensor 1 (NCS-1) may be a plausible target for $\mathrm{Pb}^{2+}$ neurotoxicity. NCS-1 was associated with numerous neuronal processes, including regulation of neurotransmission, nuclear $\mathrm{Ca}^{2+}$ regulation, cognitive functions, and neuron development through interactions with various downstream partners, including the dopamine receptors, calcineurin, and phosphatidylinositol-4 kinase-IIIb (Gorkhali, Huang et al. 2016, Boeckel, Ehrlich 2018). Considering high sequence identity of the C-terminal domain of neuronal calcium sensors (Ames, Lim 2012) and significant amount of $\mathrm{Pb}^{2+}$ in the brain (0.52-4 ppm) due to occupational and environmental exposure to the $\mathrm{Pb}^{2+}$ (Barry, Mossman 1970, Grandjean 1978), especially in the hippocampus (Grandjean 1978), other members of NCS family are likely to bind $\mathrm{Pb}^{2+}$ with higher affinity than that for $\mathrm{Ca}^{2+}$ and be involved in $\mathrm{Pb}^{2+}$-induced neurotoxicity. 
Table 3.4. Equilibrium dissociation constants for presenilin-1 helix 9 "PS1HL9", Kv4.3(2-22)

"site-1", and Kv4.3(70-90) "site-2" binding to DREAM constructs.

\begin{tabular}{|c|c|c|c|}
\hline & $\begin{array}{l}\text { PS1HL9 } \\
\mathrm{K}_{\mathrm{d}}(\mu \mathrm{M})\end{array}$ & $\begin{array}{l}\text { Site-1 } \\
\mathrm{K}_{\mathrm{d}}(\mu \mathrm{M})\end{array}$ & $\begin{array}{l}\text { Site-2 } \\
\mathrm{K}_{\mathrm{d}}(\mu \mathrm{M})\end{array}$ \\
\hline $\operatorname{apoDREAM}(\Delta 64)$ & $183 \pm 12$ & $70 \pm 3$ & $\sim 500$ \\
\hline $\mathrm{Ca}^{2+} \operatorname{DREAM}(\Delta 64)$ & $0.6 \pm 0.1$ & $2.7 \pm 0.1$ & $10 \pm 1$ \\
\hline $\mathrm{Pb}^{2+} \mathrm{DREAM}(\Delta 64)$ & $2.56 \pm 0.23$ & $5.9 \pm 0.6$ & $10.61 \pm 0.40$ \\
\hline \multicolumn{4}{|c|}{$\begin{array}{l}\text { Determined using steady-state anisotropy change. Data were fitted to Equation 2. Data for } \\
\text { apoDREAM }(\Delta 64) \text { and } \mathrm{Ca}^{2+} \mathrm{DREAM}(\Delta 64) \text { are from reference (Pham, Miksovska 2016) and } \\
\text { (Gonzalez, Pham et al. } 2014) \text {, respectively. Titrations of } \mathrm{Pb}^{2+} \mathrm{DREAM}(\Delta 64) \text { with PS1HL9, site-1 } \\
\text { and site- } 2 \text { were conducted in triplicate. Average } \mathrm{K}_{\mathrm{d}} \text { values are reported and standard deviations are } \\
\text { shown as errors. }\end{array}$} \\
\hline
\end{tabular}




\subsection{Summary}

In this report, we demonstrate that $\mathrm{Pb}^{2+}$ binds to EF-hands in DREAM for the first time. Based on fluorescence emission, circular dichroism, ITC, and fluorescence lifetime data, we provide compelling evidence that $\mathrm{Pb}^{2+}$ is able to induce structural changes analogous to $\mathrm{Ca}^{2+}$ binding. $\mathrm{Pb}^{2+}$ binding to DREAM also alters DREAM interaction with intracellular partners, such as presenilin-1 and site- 1 and site- 2 of potassium voltage channel. These results imply that DREAM and possibly other neuronal calcium sensors bind $\mathrm{Pb}^{2+}$ with a high affinity in vivo, and $\mathrm{Pb}^{2+}$ interactin with neuronal calcium sensors could explain lead-induced neurotoxicity. 


\section{CADMIUM ASSOCIATION TO DREAM PROMOTES DREAM INTERACTIONS WITH INTRACELLULAR PARTNERS IN A SIMILAR MANNER AS ITS PHYSIOLOGICAL LIGAND, CALCIUM.}

\subsection{Background and significance}

$\mathrm{Cd}^{2+}$ has been recognized as one of the most toxic environmental and industrial pollutants which exerts adverse health effects in general population because of its prolonged half-life in human (15-20 years) and low rate of excretion from the human body (Järup, Åkesson 2009, Roels, Bernard et al. 1993, Satarug, Moore 2004, Jin, Lu et al. 1998). It has been assigned as class I carcinogen by the International Agency for Research on Cancer (IARC Working Group on the Evaluation of the Carcinogenic Risk of Chemicals to Humans 1993). Occupational and as well as non-occupational exposure, including consumption of tobacco products, food, and dietary intake (Satarug, Moore 2004, IARC Working Group on the Evaluation of the Carcinogenic Risk of Chemicals to Humans 1993, IPCS 1992) to $\mathrm{Cd}^{2+}$ has been associated with different cancers, including stomach, testis, renal and others (Waalkes 2000, Pesch, Haerting et al. 2000, Joseph, Muchnok et al. 2001). The central nervous system is also susceptible to $\mathrm{Cd}^{2+}$ toxicity (Cao, Chen et al. 2009, Lopez, Figueroa et al. 2003), especially in children, elderly people, and pregnant women. Elevated concentration of $\mathrm{Cd}^{2+}$ has been reported in children with mental retardation and learning difficulties compared with a reference group (Pihl, Parkes 1977, Marlowe, Errera et al. 1983, Capel, Pinnock et al. 1981). Other studies have shown that $\mathrm{Cd}^{2+}$ concentration in hair is inversely related to the intelligent quotient (Thatcher, Lester et al. 1982, Thatcher, McAlaster et al. 1984). An 
increasing number of clinical investigations have suggested that $\mathrm{Cd}^{2+}$ intoxication is a probable etiological factor of neurodegenerative diseases, such as Parkinson's disease, Huntington's disease, and Alzheimer's disease (Okuda, Iwamoto et al. 1997, Johnson 2001, Panayi, Spyrou et al. 2002). The mechanism through which $\mathrm{Cd}^{2+}$ exerts its toxic effects remains unresolved. Oxidative stress, interference with $\mathrm{Ca}^{2+}$ - and $\mathrm{Zn}^{2+}$-dependent processes, epigenetic modification, apoptosis induction, and effects on neurotransmitters were proposed to be the underlying mechanism (Wang, Du 2013, Méndez-Armenta, Rios 2007). Animal model studies have shown that $\mathrm{Cd}^{2+}$ exposure has been associated with increased production of reactive oxygen species which in turns results in increased lipid peroxidation, altered calcium homeostasis, and DNA damage (Méndez-Armenta, Villeda-Hernández et al. 2003, ElMaraghy, Gad et al. 2001, Kumar, Agarwal et al. 1996, Lopez, Arce et al. 2006, Manca, Ricard et al. 1991). Even at relatively low concentrations, $\mathrm{Cd}^{2+}$ inhibits all known pathways of $\mathrm{Ca}^{2+}$ influx and thereby interferes with the $\mathrm{Ca}^{2+}$ homeostasis (MéndezArmenta, Rios 2007). Yoshida (Yoshida 2001) has shown that $\mathrm{Cd}^{2+}$-exposed neurons have a dose-dependent elevated concentration of cytoplasmic and nuclear $\mathrm{Ca}^{2+}$. Another study has shown that $50 \mu \mathrm{M} \mathrm{Cd}^{2+}$ inhibits $\mathrm{Ca}^{2+}$ influx into hepatocytes through receptor-operated $\mathrm{Ca}^{2+}$-channels (Thevenod, Jones 1992). The central nervous system is highly sensitive to $\mathrm{Cd}^{2+}$ exposure as 24 -hour exposure to $\mathrm{Cd}^{2+}$ results in a decrease in dopamine, serotonin, and norepinephrine concentration (Lafuente, Gonzalez-Carracedo et al. 2003, Lafuente, Fenández-Rey et al. 2001). Additionally, experimental studies on rabbits and newborn rats exposed to an elevated $\mathrm{Cd}^{2+}$ concentration have demonstrated extensive hemorrhages in the 
cerebral and cerebellar cortices, neuroglial cells with cytolysis, altered Purkinje cells, and several pyramidal cells with pyknotic nuclei (Gabbiani, Baic et al. 1967, Wong, Klaassen 1982). Studies on mice have demonstrated that $\mathrm{Cd}^{2+}$ exposure impairs hippocampus-dependent memory and learning (Wang, Zhang et al. 2017). Rigon et al. (Rigon, Cordova et al. 2008) and Kanter et al. (Kanter, Unsal et al. 2016) have shown that $\mathrm{Cd}^{2+}$ administration causes a decrease in cell vitality and neuronal apoptosis in the hippocampus in a rat model.

$\mathrm{Cd}^{2+}$ has been shown to occupy $\mathrm{Ca}^{2+}$ binding site of $\mathrm{CaM}$ (Forsén, Thulin et al. 1980, Andersson, Forsen et al. 1983) and promotes CaM association and activation of phosphodiesterase and myosin light chain kinase (Suzuki, Chao et al. 1985, Chao, Bu et al. 1995, Mazzei, Girard et al. 1984). Suzuki et al. (Suzuki, Chao et al. 1985) and Mazzei et al. (Mazzei, Girard et al. 1984) postulated that $\mathrm{Cd}^{2+}$ competition with $\mathrm{Ca}^{2+}$ for EF-hands on CaM is the key of CaM toxicity at the molecular level. Studies on protein kinase $\mathrm{C}$ have shown that $\mathrm{Cd}^{2+}$ binding to protein kinase $\mathrm{C}$, displaces $\mathrm{Zn}^{2+}$ from the regulatory domain and facilitates binding of protein kinase $\mathrm{C}$ to a nuclear protein (Block, Freyermuth et al. 1992). Other studies have shown that $\mathrm{Cd}^{2+}$ can displace $\mathrm{Ca}^{2+}$ from its binding sites in troponin $\mathrm{C}$, the calcium-binding subunit of the muscle protein complex troponin (Ellis, Strang et al. 1984, TELEMAN, DRAKENBERG et al. 1983).

Neuronal calcium sensor (NCS) is a subgroup of EF-hand superfamily that includes potassium channel-interacting protein $1-4$, recoverin, NCS-1, GCAP, and neurocalcin. These proteins are mainly expressed in the brain and retina where they transduce $\mathrm{Ca}^{2+}$ signals and regulate a myriad of intracellular processes (Ames, Lim 
2012, Burgoyne, Weiss 2001, Burgoyne 2007). Even though NCS proteins share comparatively low sequence similarity with $\mathrm{CaM}$, they are composed of four EFhands analogous to $\mathrm{CaM}$ and are sensitive to changes in intracellular $\mathrm{Ca}^{2+}$ concentration (McCue, Haynes et al. 2010). Downstream regulatory element antagonist modulator (DREAM), also known as potassium channel-interacting protein 3 (KChIP3) or calsenilin, is a $29 \mathrm{kDa}$ NCS protein that is expressed in the hippocampus and has been shown to regulate numerous cellular processes. DREAM is the first $\mathrm{Ca}^{2+}$-regulated transcriptional repressor (Carrion, Link et al. 1999). In the $\mathrm{Ca}^{2+}$-free state, DREAM binds to the downstream regulatory element (DRE) of human prodynorphin and c-fos genes and consequently represses transcription, whereas $\mathrm{Ca}^{2+}$ binding alters protein oligomerization resulting in decreased affinity for DRE sequence (Carrion, Link et al. 1999, Cheng, Pitcher et al. 2002). Prodynorphin and c-fos genes have been associated with apoptosis, pain modulation, and cell homeostasis (Costigan, Woolf 2002, Fontán-Lozano, Romero-Granados et al. 2009). It has been demonstrated that in the presence of $\mathrm{Ca}^{2+}$, DREAM interaction with carboxy-terminal fragment (CTF) of presenilin-1 (PS1) and presenilin-2 (PS2) stimulates the activity of $\gamma$ - secretase, which facilitates cleavage of amyloid precursor protein and eventually overproduces $A \beta 42$ peptide, a peptide associated with Alzheimer's disease (Buxbaum, Choi et al. 1998, Jo, Jang et al. 2005). DREAM also associates with the $\mathrm{T} 1$ domain of potassium channels and regulates their translocation to the membrane, $\mathrm{K}^{+}$current, and gating properties (An, Bowlby et al. 2000). DREAM has been linked to memory and learning processes by acting as a transcriptional repressor of CREM in a calcium-dependent fashion (Fontán-Lozano, 
Romero-Granados et al. 2009). Study of Rashid et al. (Abd Rashid, Hapidin et al. 2017) proposed that DREAM protein may participate in the mechanism of nicotine treatment-prevented learning and memory impairment in REM sleep-deprived rats by changing its expression level in the hippocampus. Recent studies have shown that $\mathrm{Ca}^{2+}$ bound DREAM binds to CaM and the formation of DREAM:CaM complex eliminates nonspecific interaction between DREAM and DRE sites and increases activation of calcineurin (Gonzalez, Arango et al. 2015, Ramachandran, Craig et al. 2012).

The three-dimensional NMR structure of $\mathrm{N}$-terminal truncated $\mathrm{Ca}^{2+}$-bound DREAM (residue 78-256) (Lusin, Vanarotti et al. 2008), shown in Figure 4.1, reveals that DREAM possesses two domains, with each domain comprising of two EF-hands. EF-1 (residue 90-119) and EF-2 (residue 128-157) along with a short N-

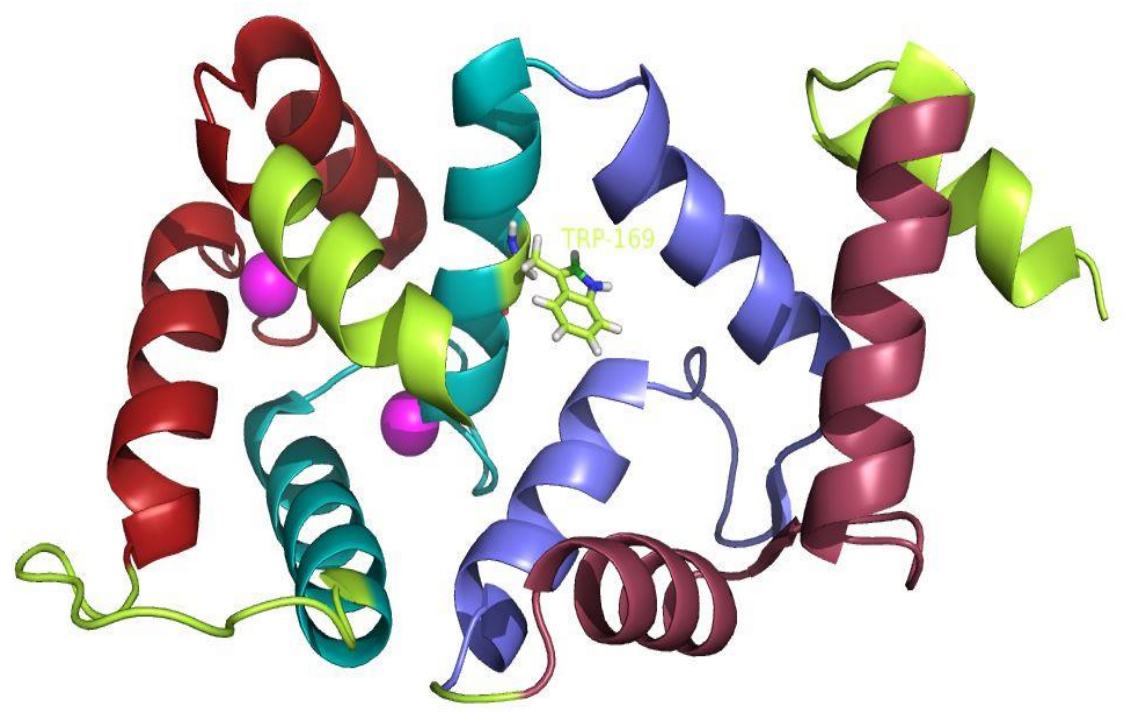

Figure 4.1. NMR structure of $\mathrm{Ca}^{2+}$-bound DREAM monomer (PDB entry 2JUL). The four EF-hands of DREAM are shown in raspberry (EF-hand 1), slate (EF-hand 2), teal (EF-hand 3), and firebrick (EF-hand 4); $\mathrm{Ca}^{2+}$ ions are shown as magenta spheres; W169 is shown as limon stick; and rest of the protein is shown in limon. 
terminus $\alpha$-helix form an $\mathrm{N}$-terminal domain, whereas EF-3 (residue 163-192) and EF-4 (residue 211-240) combined with a 13-residue long C-terminal $\alpha$-helix form a C-terminal domain. Among four EF-hands of DREAM, EF-hand 1 is not functional because the presence of the CPXG sequence at the EF-hand loop distorts $\mathrm{Ca}^{2+}-$ binding geometry. The presence of Asp at the $12^{\text {th }}$ position of the EF-2 loop makes this EF-hand specific for $\mathrm{Mg}^{2+}$ and EF-3 and EF-4 bind $\mathrm{Ca}^{2+}$ with a $\mathrm{K}_{\mathrm{d}}$ of 1-10 $\mu \mathrm{M}$ (Gifford, Walsh et al. 2007, Osawa, Dace et al. 2005, Osawa, Tong et al. 2001). We have recently shown that the EF-3 and EF-4 do not exclusively bind $\mathrm{Ca}^{2+}$ as they can also bind non-physiological metals $\mathrm{Tb}^{3+}$ and $\mathrm{Pb}^{2+}$ with an affinity that is superior to that for $\mathrm{Ca}^{2+}$ (Azam, Miksovska 2018, Gonzalez, Ramos et al. 2016).

\subsection{Results and discussion}

4.2.1 $\mathrm{Cd}^{2+}$ binding promotes the structural reorganization of DREAM.

A single tryptophan residue (W169) in WTDREAM $(\Delta 64)$ sequence is located between the $\mathrm{N}$ - and $\mathrm{C}$-terminal domain and its emission properties can be used to probe inter-domain structural changes associated with the metal binding to DREAM. It has been shown previously that in the presence of $\mathrm{Ca}^{2+}$, W169 emission intensity decreases and the emission spectrum undergoes a concomitant shift to the lower wavelength of $330 \mathrm{~nm}$ (Pham, Dhulipala et al. 2015). Close inspection of WTDREAM $(\Delta 64)$ and WTDREAM $(\Delta 160)$ structures reveals that four charged amino acid residues (K168, N172, E165, and E253) are located within $6 \AA$ of the indole ring of W169 and may serve as Trp emission quenchers. The decrease in emission intensity can be explained by a model in which $\mathrm{Ca}^{2+}$ binding causes structural rearrangement of the protein that brings the quencher amino acid 
residue(s) closer to the indole ring of W169 resulting in a decrease of tryptophan emission intensity. The blue shift of emission maximum upon $\mathrm{Ca}^{2+}$ binding is indicative of tryptophan being located in a less polar environment in the $\mathrm{Ca}^{2+}$ bound
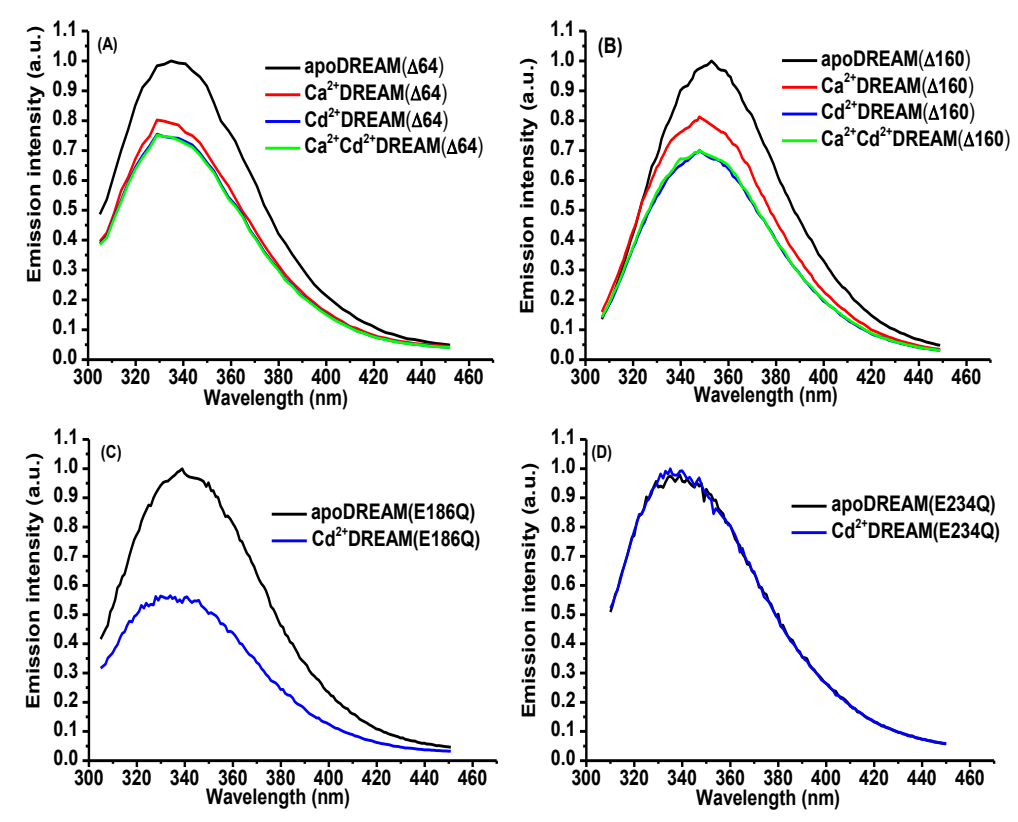

Figure 4.2. Effects of $\mathrm{Ca}^{2+}$ and $\mathrm{Cd}^{2+}$ on emission properties of W169 in (A) $\operatorname{DREAM}(\Delta 64)$, (B) $\operatorname{DREAM}(\Delta 160)$, (C) DREAM(E186), and (D) DREAM(E234Q). Conditions: $20 \mu \mathrm{M}$ protein in $20 \mathrm{mM}$ Tris $\mathrm{pH} 7.4,2 \mathrm{mM}$ EDTA or $2 \mathrm{mM} \mathrm{Ca}^{2+}$, and/or $100 \mu \mathrm{M} \mathrm{Cd}^{2+}$. Emission spectra were recorded using $295 \mathrm{~nm}$ excitation light.

state. To investigate whether $\mathrm{Cd}^{2+}$ binds to WTDREAM $(\Delta 64)$, we measured emission spectra of WTDREAM $(\Delta 64)$ in the presence of $\mathrm{Cd}^{2+}$ (Figure 4.2A). Trp at the emission maximum of $335 \mathrm{~nm}$ decreases by $\sim 25 \%$ upon addition of $\mathrm{Cd}^{2+}$ and the emission maximum shifts to $329 \mathrm{~nm}$, suggesting structural reorganization at the interface of EF-2 and EF-3 analogous to rearrangements triggered by the $\mathrm{Ca}^{2+}$ binding. Interestingly, $\mathrm{Cd}^{2+}$ association with $\operatorname{WTDREAM}(\Delta 64)$ leads to a more 
pronounced decrease in emission intensity than observed for $\mathrm{Ca}^{2+}$ association. The addition of $\mathrm{Cd}^{2+}$ to $\mathrm{Ca}^{2+}$ bound WTDREAM $(\Delta 64)$ results in an emission spectrum identical to $\mathrm{Cd}^{2+}$ bound WTDREAM $(\Delta 64)$, indicating $\mathrm{Cd}^{2+}$ binds to DREAM with a higher affinity and displaces $\mathrm{Ca}^{2+}$ from the EF-hands.

Because the EF-3 and EF-4 of DREAM bind $\mathrm{Ca}^{2+}$, it is expected that the EF-3 and EF-4 may also bind $\mathrm{Cd}^{2+}$ as $\mathrm{Cd}^{2+}$ shares similar physical properties with $\mathrm{Ca}^{2+}$. To investigate whether $\mathrm{Cd}^{2+}$ binds at EF-3 and EF-4, first, we measured emission spectra of WTDREAM $(\Delta 160)$, a protein construct that lacks EF-1 and EF2, under identical conditions as for WTDREAM $(\Delta 64)$ (Figure 4.2B). $\mathrm{Cd}^{2+}$ association causes a decrease in emission intensity analogous to $\mathrm{Ca}^{2+}$ binding, suggesting that $\mathrm{Cd}^{2+}$ binds at $\mathrm{EF}-$ hand in the $\mathrm{C}$ - terminal domain. The bathochromic shift of the emission maximum in WTDREAM $(\Delta 160)\left(\lambda_{\max }=353 \mathrm{~nm}\right)$ construct compared to WTDREAM $(\Delta 64)\left(\lambda_{\max }=335 \mathrm{~nm}\right)$ indicates that W169 sidechain in WTDREAM $(\Delta 160)$ is located in the more polar environment due to the absence of the $\mathrm{N}$ terminal domain.

To provide additional insight into $\mathrm{Cd}^{2+}$ interaction with DREAM, we prepared DREAM(E186Q) and DREAM(E234Q) constructs with EF-3 and EF-4, respectively, being nonfunctional due to the replacement of Glu residue at the $12^{\text {th }}$ position of EF-hand loop. Upon $\mathrm{Cd}^{2+}$ addition to DREAM(E186Q), the emission intensity decreases in a similar way as observed for WTDREAM $(\Delta 64)$ and WTDREAM $(\Delta 160)$, suggesting that $\mathrm{Cd}^{2+}$ binds at EF-4 and triggers structural changes that modulate emission properties of W169 (Figure 4.2C). Interestingly, the 
emission spectrum of $\mathrm{Cd}^{2+}$ bound DREAM(E234Q) is nearly identical to that for apoDREAM(E234Q) (Figure 4.2D). Although these results may suggest that EF-4 does not bind $\mathrm{Cd}^{2+}$, at least at metal concentrations probed here, this interpretation is inconsistent with the data obtained for $\mathrm{Cd}^{2+}$ binding to DREAM(234Q):1,8-ANS complex and with the ITC results as shown below. The nearly identical emission spectra of apoDREAM(E234Q) and $\mathrm{Cd}^{2+} \operatorname{DREAM}(\mathrm{E} 234 \mathrm{Q})$ suggest that $\mathrm{Cd}^{2+}$ association to EF-3 does not promote structural changes that impact the emission properties of W169. This is consistent with the molecular dynamic data (Pham, Dhulipala et al. 2015) as the superposition of MD simulations structures of apoDREAM and $\mathrm{Ca}^{2+}$ DREAM monomer reveals that the EF-3 undergoes a minor reorientation upon $\mathrm{Ca}^{2+}$ association, whereas $\mathrm{Ca}^{2+}$ binding to EF-4 leads to repositioning of $\alpha$-helices from a perpendicular to parallel orientation and displacement of $\alpha$-helix 10 . The decrease in the emission intensity of W169 upon $\mathrm{Ca}^{2+}$ or $\mathrm{Cd}^{2+}$ association can be explained with a model in which $\mathrm{Ca}^{2+}$ or $\mathrm{Cd}^{2+}$ binding to EF-4 bring the residue E253, located in $\alpha$-helix 10, closer to the indole ring of W169, resulting in more efficient quenching of W169 emission.

To determine the dissociation constant for $\mathrm{Cd}^{2+}$ binding to DREAM, $\mathrm{Cd}^{2+}$ stock was titrated into DREAM $(\Delta 64)$ protein in the presence of $100 \mu \mathrm{M} \mathrm{Ca}^{2+}($ Figure 4.3). Data were fitted to Equation 1 which reveals that $\mathrm{Cd}^{2+}$ binds to DREAM protein with an apparent dissociation constant $8 \pm 1 \mu \mathrm{M}$. Substituting the value of apparent dissociation constant in Equation 3, equilibrium dissociation constant for $\mathrm{Cd}^{2+}$ binding to DREAM protein determined to be $80 \mathrm{nM}$. 

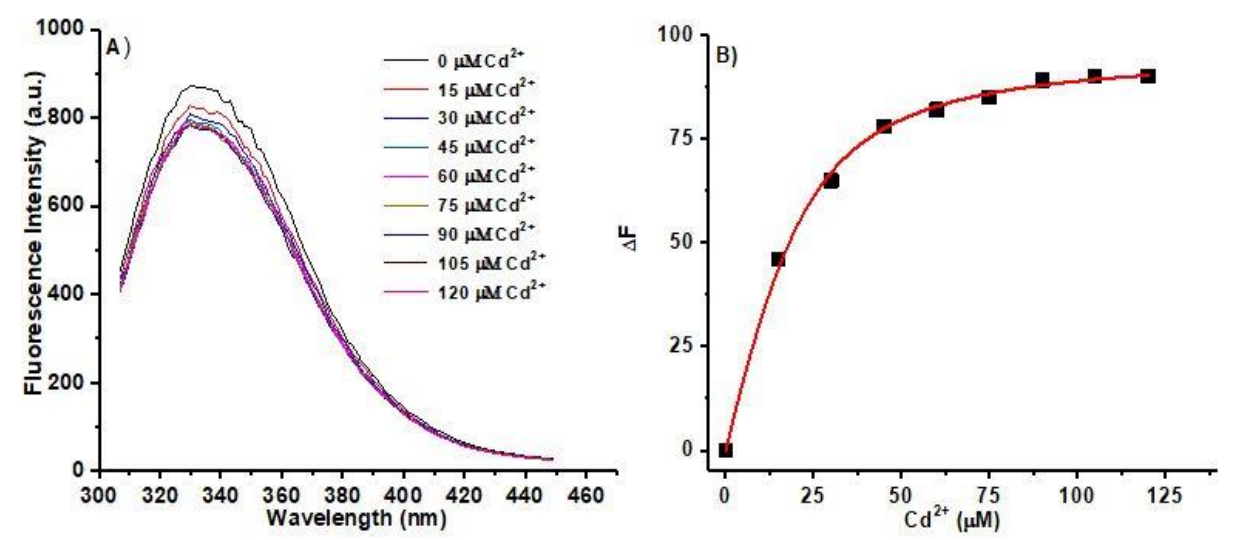

Figure 4.3. Emission spectra of $\operatorname{DREAM}(\Delta 64)$ as a function of increasing concentration of $\mathrm{Cd}^{2+}$. Conditions: $20 \mu \mathrm{M}$ WTDREAM $(\Delta 64)$ in $20 \mathrm{mM}$ Tris in presence of $100 \mu \mathrm{M} \mathrm{Ca}^{2+}$. The emission spectra were recorded using 295 $\mathrm{nm}$ excitation (A). The decrease in the emission intensity of $\operatorname{DREAM}(\Delta 64)$ due to the $\mathrm{Cd}^{2+}$ binding to $\mathrm{Ca}^{2+}$ DREAM. The experimental data were fitted using Equation 1 for obtaining equilibrium dissociation constant (B).
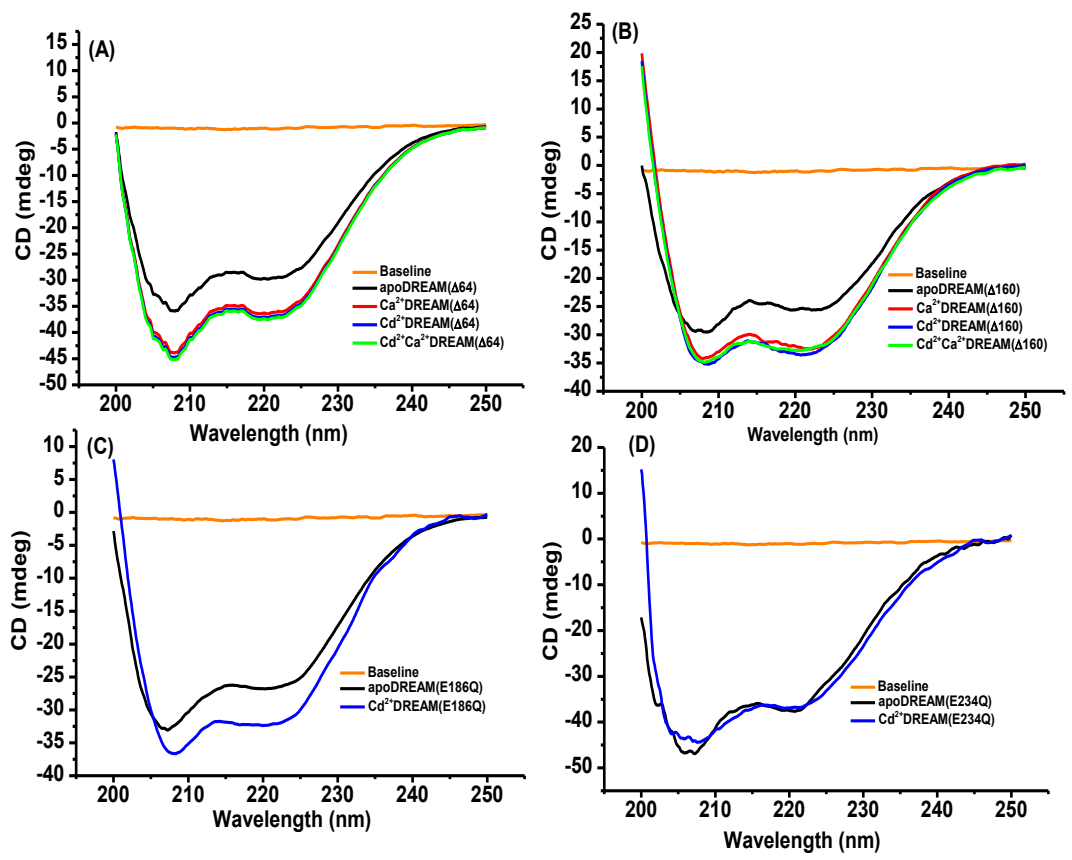

Figure 4.4. Far-UV CD spectra of (A) $20 \mu \mathrm{M}$ DREAM( $\Delta 64)$, (B) $20 \mu \mathrm{M}$ $\operatorname{DREAM}(\Delta 160), \quad(\mathrm{C}) \quad 20 \mu \mathrm{M} \quad \operatorname{DREAM}(\mathrm{E} 186)$, and (D) $20 \mu \mathrm{M}$ DREAM(E234Q). Conditions as described in Figure 4.2. 
The structural reorientation of DREAM upon $\mathrm{Cd}^{2+}$ association was also determined by far-UV CD spectroscopy. CD data presented in Figure 4.4 show that that CD signal at $208-222 \mathrm{~nm}$ decreases upon $\mathrm{Cd}^{2+}$ binding to WTDREAM $(\Delta 64)$, WTDREAM $(\Delta 160)$, and DREAM(E186Q) (Figure 4.4A-C). The decrease in the $\mathrm{CD}$ signal at 220 and $208 \mathrm{~nm}$ indicates an increase in an $\alpha$-helical content and/or reorganization of $\alpha$-helices. Similar CD spectra of WTDREAM $(\Delta 64)$ constructs in $\mathrm{Ca}^{2+}$ - and $\mathrm{Cd}^{2+}$-bound form suggests that binding of both metals causes analogous secondary structural rearrangement, which is consistent with Trp emission results. Also, the CD signal of DREAM(E234Q) is not affected by $\mathrm{Cd}^{2+}$ association to the apo-protein (Figure 4.4D). Far-UV CD data have been analyzed using secondary structure prediction tool K2D3 (Louis-Jeune, Andrade-Navarro et al. 2012) and results reveal that $\mathrm{Cd}^{2+}$ increases the $\alpha$-helical property of the protein by $\sim 6 \%$ in WTDREAM $(\Delta 64)$ and DREAM(E186Q) and by $\sim 10 \%$ in WTDREAM $(\Delta 160)$.

In addition to steady-state emission properties of W169, we have characterized W169 fluorescence in frequency-domain measurements (Figure 4.5 and Table 4.1). Modulation-phase data were analyzed using a three discrete exponential decay model. Such multi-exponential decay of the single W169 residue can be attributed to the presence of multiple rotamers of the indole side chain and/or by the presence of multiple conformational states as proposed in Ref (Pham, Dhulipala et al. 2015). The average lifetime of W169 decreases from $3.8 \mathrm{~ns}$ in apoWTDREAM $(\Delta 64)$ to $3.1 \mathrm{~ns}$ and $3.2 \mathrm{~ns}$ in $\mathrm{Ca}^{2+}$ and $\mathrm{Cd}^{2+}$ bound protein, respectively. The decrease in the average lifetime can be attributed to the decrease 
of the individual lifetimes and a concomitant increase in the pre-exponential factor $\alpha_{1}$ upon $\mathrm{Ca}^{2+}$ or $\mathrm{Cd}^{2+}$ binding to the protein (Table 4.1). Fluorescence decay parameters observed for $\operatorname{Cd}^{2+}$ WTDREAM $(\Delta 64)$ closely resembles $\mathrm{Ca}^{2+}$ WTDREAM $(\Delta 64)$, pointing towards a similar environment of $\mathrm{W} 169 \mathrm{in} \mathrm{Cd}^{2+}$ and $\mathrm{Ca}^{2+}$ bound DREAM.

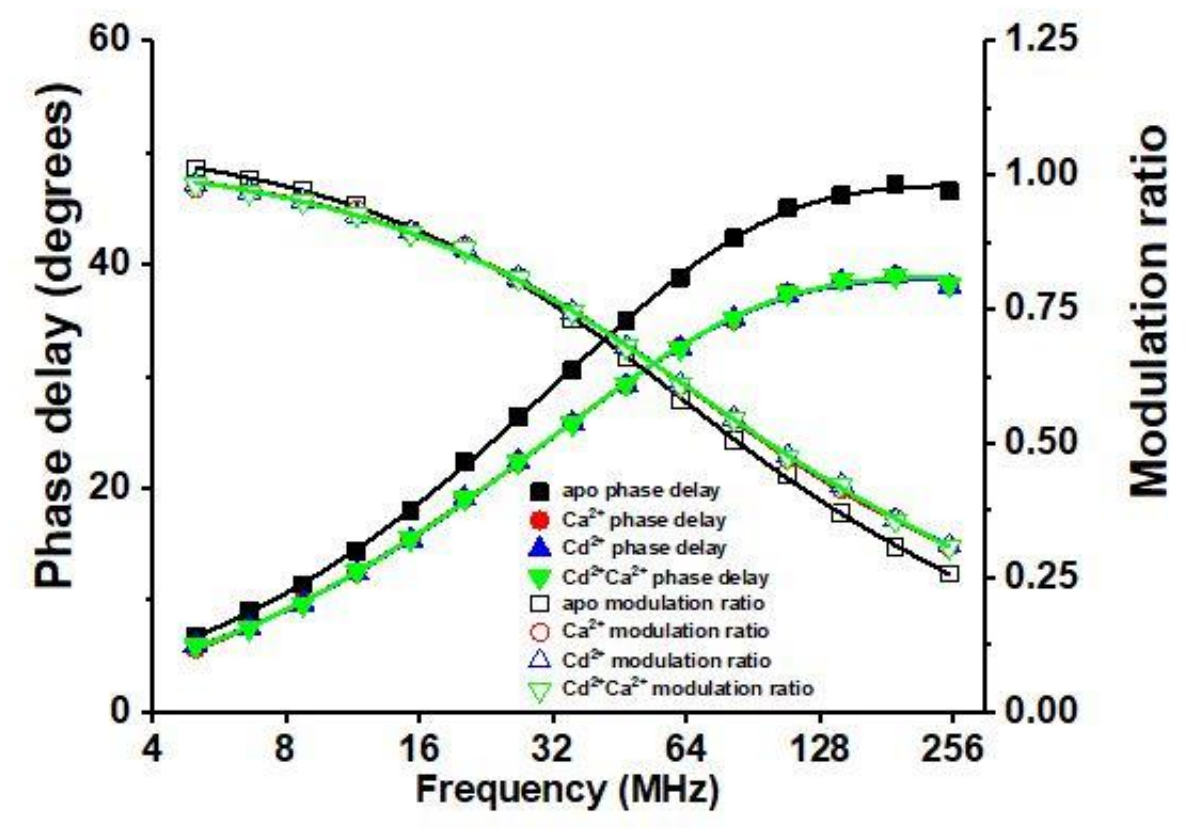

Figure 4.5. Frequency-domain intensity decay of $20 \mu \mathrm{M}$ $\operatorname{apoDREAM}(\Delta 64) \quad$ (square), $\mathrm{Ca}^{2+} \operatorname{DREAM}(\Delta 64) \quad$ (circle), $\mathrm{Cd}^{2+} \operatorname{DREAM}(\Delta 64)$ (up triangle), and $\mathrm{Cd}^{2+} \mathrm{Ca}^{2+} \operatorname{DREAM}(\Delta 64)$ (down triangle). Solid symbols represent phase delay, whereas empty symbols represent modulation ratio. Solid lines correspond to the fitting of the data using a sum of three exponential decay model. 
Table 4.1. Fluorescence decay parameters of WTDREAM $(\Delta 64)$ in the presence and absence of $\mathrm{Ca}^{2+}$ and $\mathrm{Cd}^{2+}$.

\begin{tabular}{llllllllllll}
\hline Samples & $\begin{array}{l}\tau_{1} \\
(\mathrm{~ns})\end{array}$ & $\begin{array}{l}\tau_{2} \\
(\mathrm{~ns})\end{array}$ & $\begin{array}{l}\tau_{3} \\
(\mathrm{~ns})\end{array}$ & $\begin{array}{l}\mathrm{f}_{1} \\
(\%)\end{array}$ & $\begin{array}{l}\mathrm{f}_{2} \\
(\%)\end{array}$ & $\begin{array}{l}\mathrm{f}_{3} \\
(\%)\end{array}$ & $\begin{array}{l}\alpha_{1} \\
(\%)\end{array}$ & $\begin{array}{l}\alpha_{2} \\
(\%)\end{array}$ & $\begin{array}{l}\alpha_{3} \\
(\%)\end{array}$ & $\begin{array}{l}<\tau> \\
(\mathrm{ns})\end{array}$ & $\chi^{2}$ \\
\hline apo & $0.24 \pm$ & $2.5 \pm$ & $7.1 \pm$ & $17.3 \pm$ & $45.4 \pm$ & $37.3 \pm$ & 75.1 & 19.2 & 5.9 & 3.8 & 1.5 \\
& 0.03 & 0.1 & 0.3 & 0.8 & 3.0 & 0.3 & & & & & \\
\hline $\mathrm{Ca}^{2+}$ & $0.16 \pm$ & $1.9 \pm$ & $6.3 \pm$ & $22.0 \pm$ & $37.5 \pm$ & $40.5 \pm$ & 84.5 & 12.1 & 3.3 & 3.1 & 0.9 \\
& 0.02 & 0.1 & 0.2 & 0.9 & 2.0 & 2.0 & & & & & \\
\hline $\mathrm{Cd}^{2+}$ & $0.16 \pm$ & $1.9 \pm$ & $6.3 \pm$ & $22.3 \pm$ & $37.3 \pm$ & $40.4 \pm$ & 84.6 & 12.0 & 3.2 & 3.2 & 0.6 \\
& 0.02 & 0.1 & 0.2 & 0.2 & 2.0 & 2.0 & & & & & \\
\hline $\mathrm{Cd}^{2+} \mathrm{Ca}^{2+}$ & $0.16 \pm$ & $2.0 \pm$ & $6.3 \pm$ & $22.5 \pm$ & $37.5 \pm$ & $40.0 \pm$ & 83.9 & 12.3 & 3.6 & 3.2 & 0.9 \\
& 0.02 & 0.1 & 0.3 & 0.9 & 2.0 & 2.0 & & & & & \\
\hline
\end{tabular}

Recovered from Vinci analysis software using a sum of three discrete exponential decays. Phase and modulation errors were respectively set at $\leq 0.2^{\circ}$ and $\leq 0.004 . \tau_{1}, \tau_{2}$, and $\tau_{3}$ represent lifetimes of the three discrete exponential decays; $f_{1}, f_{2}$ and $f_{3}$ are exponential decay fractions; $\alpha_{1}, \alpha_{2}$, and $\alpha_{3}$ represent pre-exponential factors. $\chi^{2}$ represents the goodness of the fit. 


\subsection{2 $\mathrm{Cd}^{2+}$ regulates DREAM affinity for 1,8-ANS}

We have shown previously that $\mathrm{Ca}^{2+}$ association increases DREAM affinity for small hydrophobic molecules such as 1,8-ANS and arachidonic acid (Gonzalez, Miksovska 2014). To probe whether $\mathrm{Cd}^{2+}$ association impacts DREAM affinity for 1,8-ANS, the emission spectra of WTDREAM $(\Delta 64)$ :1,8-ANS complex in the metal-free form and in the presence of $\mathrm{Ca}^{2+}$ or $\mathrm{Cd}^{2+}$ were determined. 1,8ANS has been extensively used for evaluating hydrophobicity of the protein surface as 1,8-ANS is nearly non-fluorescent in aqueous solutions and its emission intensity increases upon the association to hydrophobic cavities on the protein surface (Cardamone, Puri 1992). The effect of $\mathrm{Cd}^{2+}$ binding on DREAM affinity for 1,8 ANS is shown in Figure 4.6. The rise in the emission intensity of 1,8-ANS in the presence of apoWTDREAM $(\Delta 64)$ is indicative of 1,8-ANS binding to hydrophobic patches on the protein surface. The fluorescence intensity increases further upon $\mathrm{Cd}^{2+}$ addition to WTDREAM $(\Delta 64)$, due to an additional binding of 1,8 -ANS to the protein (Figure 4.6). Interestingly, the emission intensity of $\mathrm{Cd}^{2+}$ WTDREAM $(\Delta 64): 1,8$ ANS complex is lower than that measured for $\mathrm{Ca}^{2+}$ WTDREAM $(\Delta 64): 1,8$ ANS. These results suggest that overall structural changes triggered by $\mathrm{Cd}^{2+}$ binding to DREAM are somewhat limited and do not include a full exposure of the hydrophobic surface as observed in the case of $\mathrm{Ca}^{2+}$ binding. Analogous increase in emission intensity is observed for $\mathrm{Cd}^{2+}$ binding to metal-free DREAM(E186Q):1,8-ANS and DREAM(E234Q):1,8-ANS complex, indicating, that both EF-3 and EF-4 binds $\mathrm{Cd}^{2+}$ and the metal association to either EF-hand increases the accessibility of the 1,8-ANS binding sites. The emission 
intensity of the $\mathrm{Cd}^{2+}$ DREAM $(\Delta 160): 1,8$-ANS complex is comparable to that for $\mathrm{Ca}^{2+} \operatorname{DREAM}(\Delta 160): 1,8$-ANS. The emission maxima for both complexes are redshifted with respect to the emission maxima observed for $\mathrm{Cd}^{2+}$ and $\mathrm{Ca}^{2+}$ bound
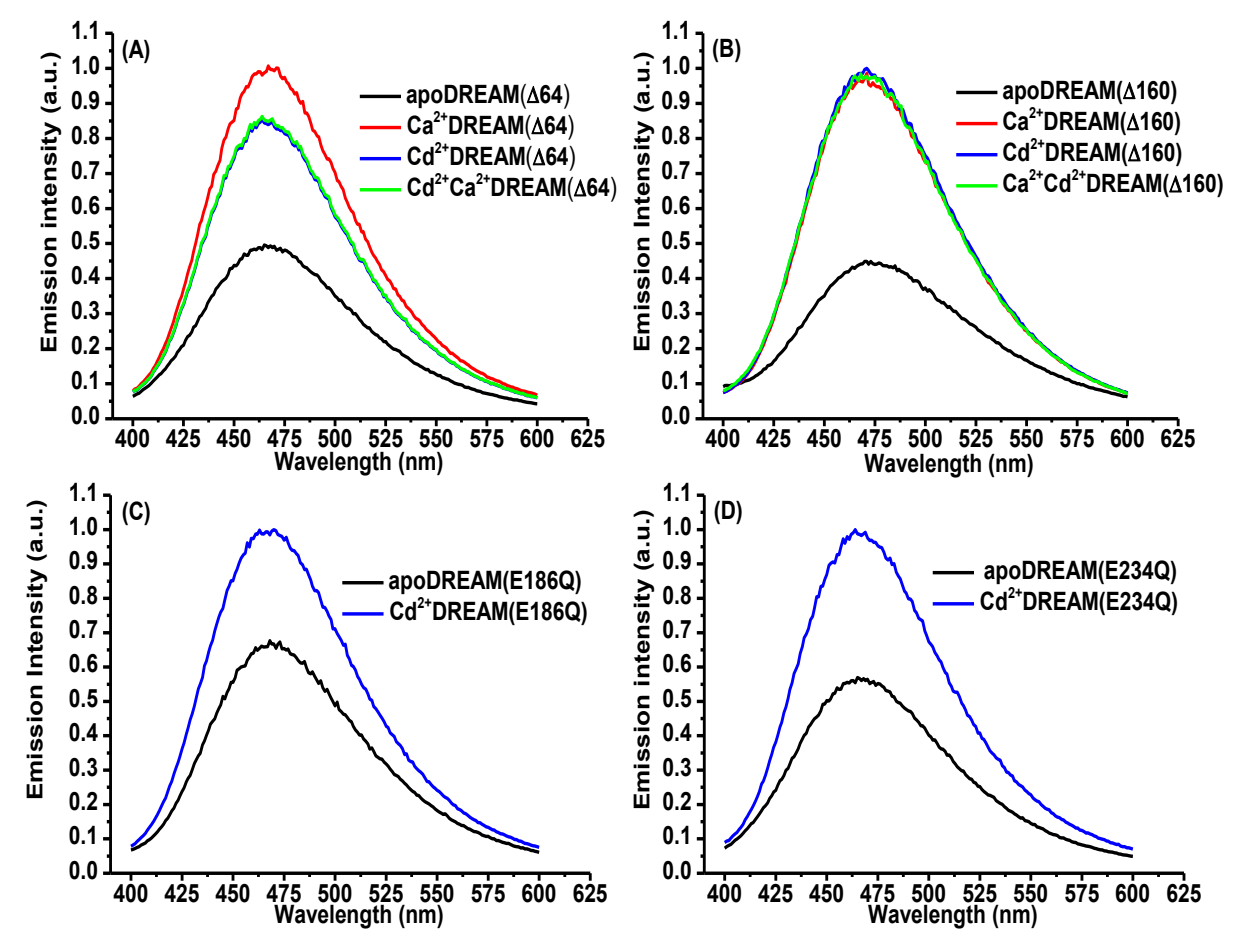

Figure 4.6. Emission spectra of (A) 1,8-ANS:DREAM( $\triangle 64)$, (B) 1,8ANS:DREAM( $(\Delta 160), \quad$ (C) 1,8-ANS:DREAM(E186), and (D) 1,8ANS:DREAM(E234Q) complexes. Conditions: Conditions: $20 \mu \mathrm{M}$ protein in $20 \mathrm{mM}$ Tris $\mathrm{pH}$ 7.4, $20 \mu \mathrm{M}$ 1,8-ANS, $2 \mathrm{mM}$ EDTA or $2 \mathrm{mM} \mathrm{Ca}^{2+}$, and/or 100 $\mu \mathrm{M} \mathrm{Cd}{ }^{2+}$. Emission spectra were recorded using $350 \mathrm{~nm}$ excitation light.

WTDREAM $(\Delta 64)$ likely due to the large solvent exposure of the hydrophobic cavity in $\operatorname{DREAM}(\Delta 160)$ construct. To determine the equilibrium affinity constant for 1,8 ANS binding to DREAM, we titrated WTDREAM $(\Delta 64)$ into a constant amount of 1,8-ANS and analyzed the experimental data using Equation 1 (Figure 4.7). Equilibrium dissociation constant recovered for 1,8-ANS binding to $\operatorname{Cd}^{2+}$ WTDREAM $(\Delta 64)\left(\mathrm{K}_{\mathrm{d}}=84 \pm 4 \mu \mathrm{M}\right)$ is two-fold lower than that previously 
determined for 1,8 -ANS binding to apoWTDREAM $(\Delta 64)(195 \pm 20 \mu \mathrm{M})$ (Gonzalez, Miksovska 2014), supporting the idea that $\mathrm{Cd}^{2+}$ association with DREAM leads to increased exposure of hydrophobic cavities. However, the $\mathrm{K}_{\mathrm{d}}$ value for 1,8-ANS binding to $\operatorname{Cd}^{2+}{ }^{2} \operatorname{WTDREAM}(\Delta 64)(84 \pm 4 \mu \mathrm{M})$ is higher than the value previously determined for ANS binding to $\mathrm{Ca}^{2+} \operatorname{WTDREAM}(\Delta 64)\left(\mathrm{K}_{\mathrm{d}}=62 \pm\right.$ $4 \mu \mathrm{M}$ ) (Gonzalez, Miksovska 2014). This is in agreement with the steady-state emission spectra that show lower emission intensity of $\mathrm{Cd}^{2+} \mathrm{WTDREAM}(\Delta 64): 1,8$ ANS compare to $\mathrm{Ca}^{2+}$ WTDREAM $(\Delta 64): 1,8$-ANS.

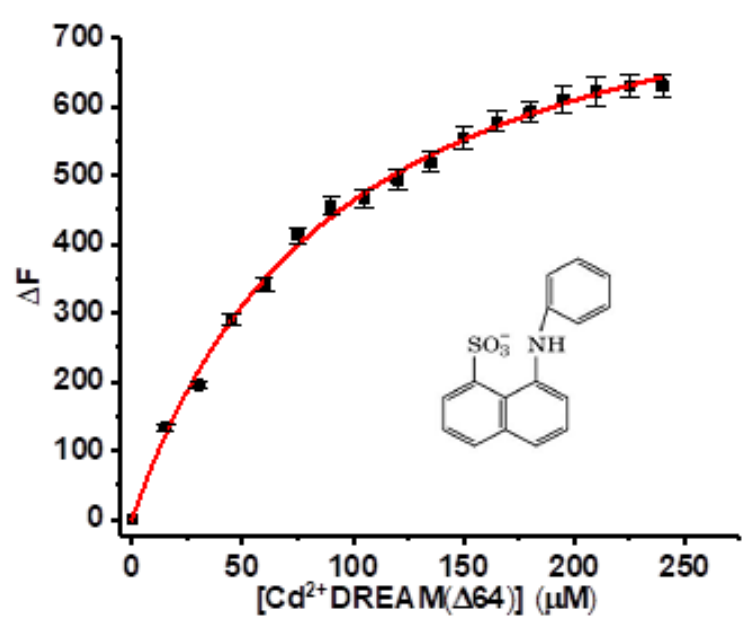

Figure 4.7. Titration curve for 1,8-ANS binding to $\mathrm{Cd}^{2+} \operatorname{DREAM}(\Delta 64)$. The solid line represents the best fit of the experimental data using Equation 1. Standard deviations are shown as error bars.

To corroborate the findings of the WTDREAM $(\Delta 64)$ :1,8-ANS steadystate emission study, we measured the lifetime of WTDREAM $(\Delta 64): 1,8$-ANS in metal-free form and in the presence of $\mathrm{Cd}^{2+}$ or $\mathrm{Ca}^{2+}$ (Figure 4.8 and Table 4.2). The fluorescence decay parameters for 1,8 -ANS bound to $\operatorname{WTDREAM}(\Delta 64)$ are sensitive to metal-induced structural rearrangement. 1,8-ANS in $20 \mathrm{mM}$ Tris shows 


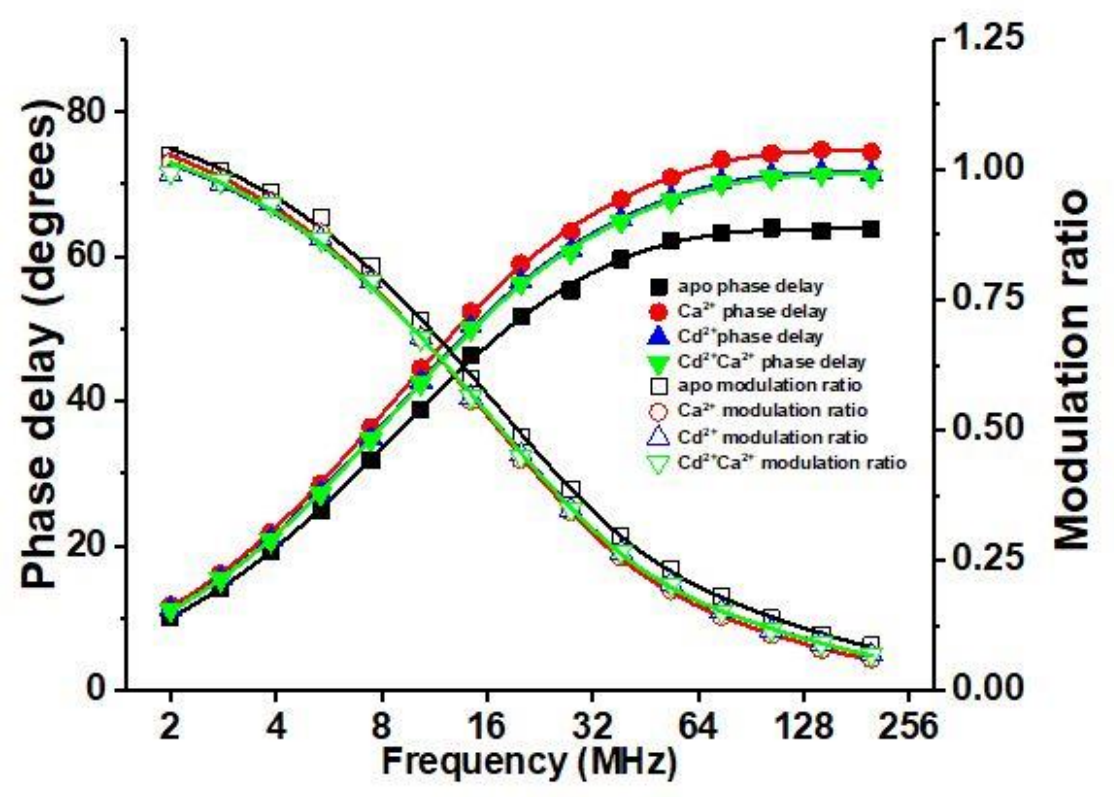

Figure 4.8 Frequency-domain intensity decay of $40 \mu \mathrm{M}$ 1,8-ANS:54 $\mu \mathrm{M}$ DREAM $(\Delta 64)$ complexes in the presence of EDTA (squares), $\mathrm{Ca}^{2+}$ (circle), $\mathrm{Cd}^{2+}$ (up triangle), and $\mathrm{Cd}^{2+} \mathrm{Ca}^{2+}$ (down triangle). Solid symbols represent phase delay, whereas empty symbols represent modulation ratio. Solid lines represent the fitting of the data using three-exponential decay model.

a single lifetime of $0.28 \mathrm{~ns}$, but upon binding to metal-free WTDREAM $(\Delta 64)$ two additional lifetimes $\left(\tau_{1}=4.1 \mathrm{~ns}\right.$ and $\left.\tau_{2}=16.9 \mathrm{~ns}\right)$ were resolved, which can be attributed to 1,8 -ANS association to two distinct 1,8-ANS binding sites on DREAM surface. Previously, we used a molecular docking approach to identify 1,8-ANS binding sites (Gonzalez, Miksovska 2014) and attributed the shorter lifetime $\left(\tau_{1}=\right.$ $4.1 \mathrm{~ns}$ ) to 1,8 -ANS association to a partially solvent-exposed binding site between the EF-3 and EF-4. The longer lifetime $\left(\tau_{2}=16.9 \mathrm{~ns}\right)$ can be assigned to $1,8-\mathrm{ANS}$ binding to a nonpolar solvent restricted binding site between helix-8 and helix-9 of EF-4. $\mathrm{Cd}^{2+}$ addition to metal-free WTDREAM( $\left.\Delta 64\right): 1,8-\mathrm{ANS}$ results in an increase 
in the longer lifetime ( $\left.\tau_{2}=17.8 \mathrm{~ns}\right)$ and the $\alpha_{2}$ parameter increases from $21.9 \%$ for metal-free WTDREAM $(\Delta 64): 1,8$-ANS complex to $36.7 \%$ for $\mathrm{Cd}^{2+}$ WTDREAM( $(464): 1,8$-ANS complex (Table 4.2). Also, $\mathrm{Ca}^{2+}$ binding to metalfree WTDREAM $(\Delta 64): 1,8$-ANS complex leads to more pronounced increase in preexponential factor $\alpha_{2}$ than $\mathrm{Cd}^{2+}$ association, in agreement with the results from the emission data that show higher emission intensity of WTDREAM $(\Delta 64): 1,8$-ANS complex in the presence of $\mathrm{Ca}^{2+}$ with respect to $\mathrm{Cd}^{2+}$ (Table 4.2). The small alteration of individual WTDREAM:1,8-ANS complex lifetimes upon $\mathrm{Ca}^{2+}$ or $\mathrm{Cd}^{2+}$ association compare to metal-free $\operatorname{WTDREAM}(\Delta 64)$ is indicative of 1,8 -ANS binding to identical binding sites in metal-free, $\mathrm{Cd}^{2+}$, and $\mathrm{Ca}^{2+}$-bound protein (Table $4.2)$. 
Table 4.2. Fluorescence decay parameters of WTDREAM $(\Delta 64): 1,8$-ANS complexes.

\begin{tabular}{lcccccccc}
\hline Samples & $\tau_{1}(\mathrm{~ns})$ & $\alpha_{1}(\%)$ & $\mathrm{f}_{1}(\%)$ & $\tau_{2}(\mathrm{~ns})$ & $\alpha_{2}(\%)$ & $\mathrm{f}_{2}(\%)$ & $\alpha_{0}(\%)$ & $\chi^{2}$ \\
\hline $\mathrm{apo}$ & $4.1 \pm 0.1$ & 15.5 & $15.4 \pm 0.9$ & $16.9 \pm 0.2$ & 21.9 & $83.0 \pm 1.0$ & 62.6 & 0.9 \\
\hline $\mathrm{Ca}^{2+}$ & $4.3 \pm 0.2$ & 16.9 & $9.7 \pm 0.6$ & $18.0 \pm 0.1$ & 42.7 & $88.9 \pm 0.5$ & 40.3 & 0.9 \\
\hline $\mathrm{Cd}^{2+}$ & $4.3 \pm 0.3$ & 15.9 & $9.6 \pm 0.6$ & $17.8 \pm 0.2$ & 36.7 & $88.8 \pm 0.6$ & 47.4 & 0.8 \\
\hline $\mathrm{Cd}^{2+} \mathrm{Ca}^{2+}$ & $4.3 \pm 0.2$ & 15.9 & $9.8 \pm 0.6$ & $17.9 \pm 0.1$ & 36.8 & $88.9 \pm 0.5$ & 47.2 & 0.9
\end{tabular}

Data recovered from Vinci analysis software using a sum of three discrete exponential decays with fixed 1,8ANS lifetime $\left(\tau_{0}=0.28 \mathrm{~ns}\right.$ ). Phase and modulation errors were respectively set at $\leq 0.2^{\circ}$ and $\leq 0.004$. $\tau_{0}, \tau_{1}$, and $\tau_{2}$ represent lifetimes of the three discrete exponential decays; $f_{0}, f_{1}$ and $f_{2}$ are exponential decay fractions; $\alpha_{0}, \alpha_{1}$, and $\alpha_{2}$ represent pre-exponential factors. $\chi^{2}$ represents the goodness of the fit. 
4.2.3 $\mathrm{Cd}^{2+}$ associates with DREAM through an entropy-driven process.

The thermodynamic parameters associated with $\mathrm{Cd}^{2+}$ binding to $\mathrm{Ca}^{2+}$ WTDREAM $(\Delta 64)$ and $\mathrm{Ca}^{2+} \mathrm{WTDREAM}(\Delta 160)$ were determined using ITC (Figure 4.9). The binding isotherms were analyzed using a sequential model with two binding sites and the fitting parameters are listed in Table 4.3. $\mathrm{Cd}^{2+}$ displacement

(A)

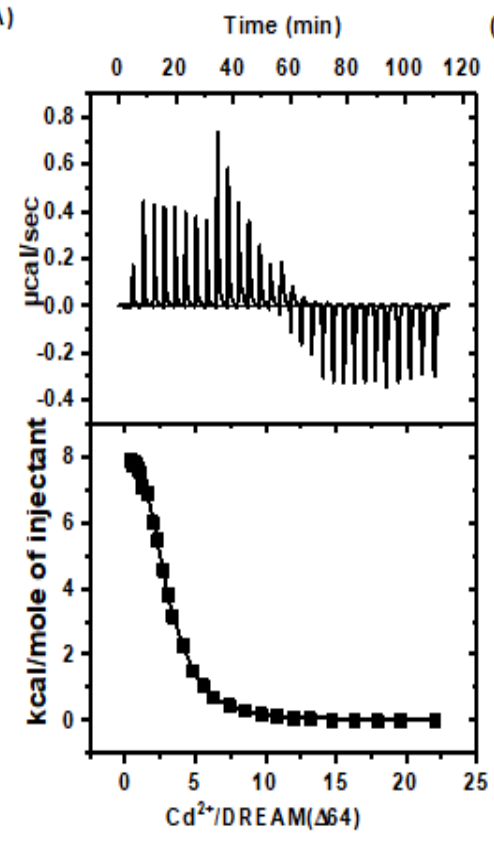

(B)

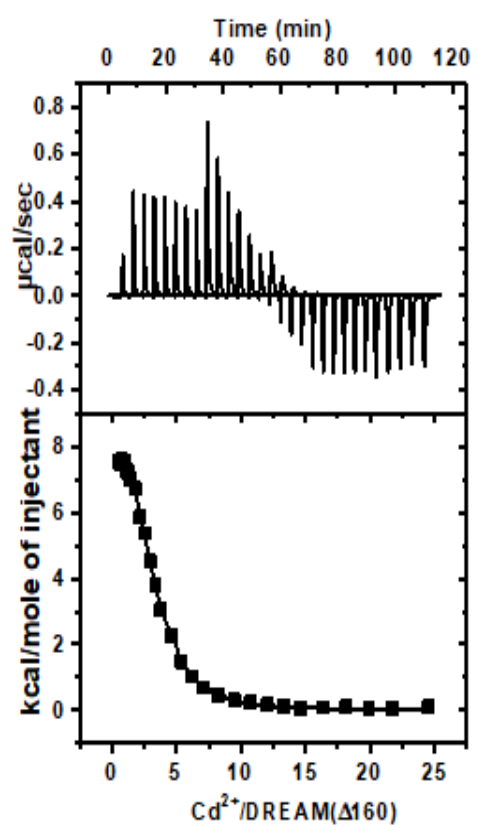

Figure 4.9. ITC isotherms for the displacement of $\mathrm{Ca}^{2+}$ by $\mathrm{Cd}^{2+}$ from EF-hand of (A) DREAM $(\Delta 64)$ and (B) DREAM $(\Delta 160)$. The upper panels of the profiles represent the thermal power expressed in $\mu \mathrm{cal} . \mathrm{s}^{-1}$, whereas the lower panels show integrated reaction heats $(\Delta \mathrm{H})$ expressed in kcal. mole ${ }^{-1}$. The solid lines represent the best fitting curve using sequential model $(2$ sites). Thermodynamics parameters recovered from the model are listed in Table 4.3.

of $\mathrm{Ca}^{2+}$ from EF-hands of WTDREAM $(\Delta 64)$ is an entropy-driven process as evidenced by a positive change in enthalpy $\left(\Delta \mathrm{H}_{\text {total }}=25 \pm 5 \mathrm{kcal} \mathrm{mol}^{-1}\right)$. Two $\mathrm{Cd}^{2+}$ ions associate with $\mathrm{Ca}^{2+}$ WTDREAM $(\Delta 64)$ with apparent association constants of 22 $\pm 4 \mathrm{M}^{-1}$ and $7 \pm 1 \mathrm{M}^{-1}$. Slightly lower affinity constants were measured for WTDREAM $(\Delta 160), \mathrm{K}_{1}=18 \pm 2 \mathrm{M}^{-1}$ and $\mathrm{K}_{2}=5 \pm 2 \mathrm{M}^{-1}$, in agreement with $\mathrm{Cd}^{2+}$ 
binding to the $\mathrm{C}$ - terminal domain. Based on these results we can not unambiguously assign the affinity constants to the individual EF-hands in the C- terminal domain. Using Equation 2 and 3, the overall apparent dissociation constant for $\mathrm{Cd}^{2+}$ binding to $\mathrm{Ca}^{2+}$ WTDREAM $(\Delta 64)$ was determined to be $9 \pm 1 \mu \mathrm{M}$ and the overall dissociation constant for $\mathrm{Cd}^{2+}$ binding to metal-free WTDREAM $(\Delta 64)$ was found to be $89 \pm 10 \mathrm{nM}$, which is in agreement with the value obtained from fluorescence titration $(80 \pm 10 \mathrm{nM})$. These results show that apoDREAM binds $\mathrm{Cd}^{2+}$ with approximately 10 -fold higher affinity than $\mathrm{Ca}^{2+}$. This is somewhat surprising, as another EF-hand protein, calmodulin, binds $\mathrm{Cd}^{2+}$ with 3-fold lower affinity than its physiological ligand $\mathrm{Ca}^{2+}$ (Milos, Schaer et al. 1989).

The $\mathrm{Cd}^{2+}$ association to both EF-hands shows a positive reaction enthalpy and entropy change. The gain of entropy over enthalpy cannot be explained in term of a water molecule(s) release as previous Raman and computational study suggested $\left[\mathrm{Cd}\left(\mathrm{OH}_{2}\right)_{6}\right]^{2+}$ clusters to be thermodynamically stable (Rudolph, Pye 1998), whereas a first hydration sphere of $\mathrm{Ca}^{2+}$ ion can include up to 8 water molecules (Jalilehvand, Spångberg et al. 2001, Marcus 1991). The X-ray structures of skeletal troponin $\mathrm{C}$ shows only small differences in the coordination of $\mathrm{Cd}^{2+}$ compared to $\mathrm{Ca}^{2+}$ with both metals adopting distorted pentagonal bipyramidal coordination (Rao, Satyshur et al. 1996). On the other hand, in the X-ray structure of the $\mathrm{N}$ - terminal domain of cardiac troponin $\mathrm{C}, \mathrm{Cd}^{2+}$ bound to $\mathrm{EF}-1$ exhibit noncanonical coordination with a Cys residue serving as a coordinating ligand (Fulcher 2015). Unlike $\mathrm{Ca}^{2+}, \mathrm{Cd}^{2+}$ is a soft metal and forms a covalent bond with thiol ligands (Choong, Liu et al. 2014). In the sequence of mouse DREAM, Cys 181 is located 
in the EF-3 loop and thus may participate in $\mathrm{Cd}^{2+}$ metal coordination. However, structural studies are necessary to conclusively identify which residues participate in $\mathrm{Cd}^{2+}$ coordination. 
Table 4.3. Thermodynamic parameters determined for displacement of $\mathrm{Ca}^{2+}$ by $\mathrm{Cd}^{2+}$ from $\mathrm{EF}$ hands of DREAM.

\begin{tabular}{lllllll}
\hline & $\begin{array}{l}\mathrm{K}_{1} \times 10^{4} \\
\left(\mathrm{M}^{-1}\right)\end{array}$ & $\begin{array}{l}\mathrm{K}_{2} \times 10^{4} \\
\left(\mathrm{M}^{-1}\right)\end{array}$ & $\begin{array}{l}\Delta \mathrm{H}_{1} \\
\left(\mathrm{kcal} \mathrm{mol}^{-1}\right)\end{array}$ & $\begin{array}{l}\Delta \mathrm{H}_{2} \\
\left(\mathrm{kcal} \mathrm{mol}^{-1}\right)\end{array}$ & $\begin{array}{l}\mathrm{T} \Delta \mathrm{S}_{1} \\
\left(\mathrm{kcal} \mathrm{mol}^{-1}\right)\end{array}$ & $\begin{array}{l}\mathrm{T} \Delta \mathrm{S}_{2} \\
\left(\mathrm{kcal} \mathrm{mol}^{-1}\right)\end{array}$ \\
\hline $\operatorname{DREAM}(\Delta 64)+\mathrm{Cd}^{2+}$ & $22 \pm 4$ & $7 \pm 1$ & $9 \pm 5$ & $17 \pm 2$ & $16 \pm 5$ & $23 \pm 2$ \\
\hline $\operatorname{DREAM}(\Delta 160)+\mathrm{Cd}^{2+}$ & $18 \pm 2$ & $5 \pm 2$ & $10 \pm 3$ & $16 \pm 4$ & $17 \pm 3$ & $22 \pm 4$ \\
\hline
\end{tabular}

All experiments were conducted three times at $25^{\circ} \mathrm{C}$. Data were fitted with a sequential model (2 sites); errors represent standard deviations from three independent measurements. 
4.2.4 $\mathrm{Cd}^{2+}$ Modulates DREAM Interactions with Binding Partners.

It has been shown previously that $\mathrm{Ca}^{2+}$ association facilitates DREAM interaction with C-terminal domain of presenilin-1 which stimulates the activity of $\gamma$ - secretase and consequently facilitates the generation of $\mathrm{A} \beta 42$, a peptide that has been linked to Alzheimer's disease (Jo, Jang et al. 2005). To investigate whether $\mathrm{Cd}^{2+}$ binding impacts DREAM affinity for presenilin, the equilibrium dissociation constant for DREAM association with PS1HL9 has been determined. We have chosen PS1HL9 as a model for presenilin-1 as earlier studies have shown that DREAM interacts with the residues from helix-9 of presenilin-1 (Buxbaum, Choi et al. 1998, Pham, Miksovska 2016). $\mathrm{Cd}^{2+}$ DREAM was titrated into the solution of FITC-tagged PS1HL9 and the change in the anisotropy was determined and plotted as a function of $\mathrm{Cd}^{2+}$ DREAM concentration (Figure 4.10). The equilibrium

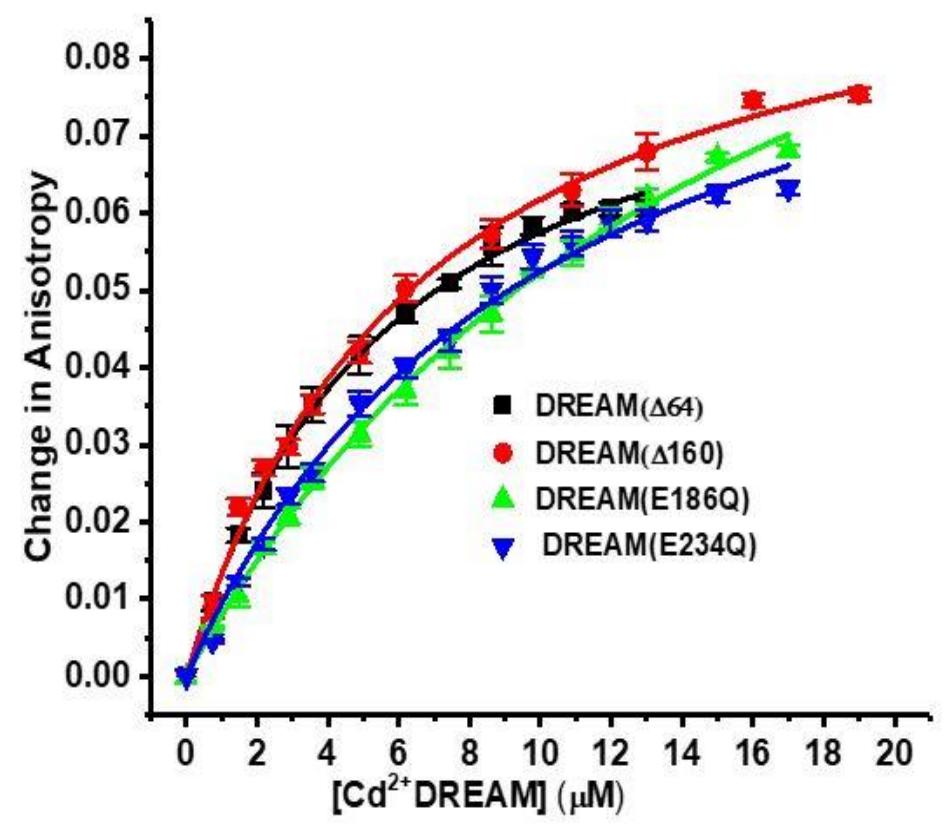

Figure 4.10 Titration of $0.5 \mu \mathrm{M}$ PS1HL9 against $\mathrm{Cd}^{2+}$-bound DREAM constructs. The solid lines represent the fits of the experimental data using Equation 1; standard deviations are shown as error bars. 
dissociation constant was obtained by fitting the titration data to Equation 1 . $\mathrm{Cd}^{2+}$ WTDREAM $(\Delta 64)$ has 10-fold lower affinity for PS1HL9 $\left(\mathrm{K}_{\mathrm{d}}=6 \pm 1 \mu \mathrm{M}\right)$ than $\mathrm{Ca}^{2+}$ WTDREAM $(\Delta 64)\left(\mathrm{K}_{\mathrm{d}}=0.6 \pm 0.1 \mu \mathrm{M}\right.$, respectively $)$ and about 30-times higher affinity for PS1HL9 than apoWTDREAM $(\Delta 64)\left(\mathrm{K}_{\mathrm{d}}=183 \pm 12 \mu \mathrm{M}\right)($ Table 4.4), suggesting $\mathrm{Cd}^{2+}$ association modulates DREAM interaction with presenilin-1. PS1HL9 binds to $\operatorname{Cd}^{2+}$ WTDREAM $(\Delta 160)$ with a $\mathrm{K}_{\mathrm{d}}$ similar to $\mathrm{Cd}^{2+}$ WTDREAM $(\Delta 64)$ confirms that N-terminal doesn't have any role in PS1HL9 binding (Table 4.4). To determine $\mathrm{Cd}^{2+}$ binding to which EF-hand regulates DREAM interactions with PS1HL9, we investigated PS1HL9 binding to $\mathrm{Cd}^{2+}$ DREAM(E186Q) and Cd ${ }^{2+}$ DREAM(E234Q). Both Cd ${ }^{2+}$ DREAM(E234Q) and $\mathrm{Cd}^{2+}$ DREAM(E186Q) have a lower affinity for PS1HL9 (Table 4.4). This finding is consistent with our previous finding that $\mathrm{Ca}^{2+} \operatorname{DREAM}(\mathrm{E} 186 \mathrm{Q})$ has a lower affinity for PS1HL9 than $\mathrm{Ca}^{2+}$ DREAM(E234Q) (Pham, Miksovska 2016). It has been shown in transgenic mice model that $\mathrm{Cd}^{2+}$ treatment increases the level of $\mathrm{A} \beta 42$ and aggravates behavioral symptoms of Alzheimer's disease ( $\mathrm{Li}, \mathrm{Lv}$ et al. 2012). The observed $\mathrm{Cd}^{2+}$ modulation of DREAM interaction with PS1HL9 indicates that at high concentration $\mathrm{Cd}^{2+}$, the formation of $\mathrm{Cd}^{2+}$ DREAM complex might facilitate of $\beta$-amyloid precursor protein cleavage to produce $A \beta 42$ and contribute to Alzheimer's disease

DREAM, as well as other members of KChIPs, are integral auxiliary subunits of A-type potassium voltage channels and binding of KChIPs to Kv4 channels has been shown to result in regulation of fast component of $\mathrm{I}_{\mathrm{TO}}$ current in heart and Iso $_{\text {So }}$ current in brain, modulation of gating properties, increased surface 
expression and facilitation of subunit assembly of Kv4 channels (An, Bowlby et al. 2000, Bourdeau, Laplante et al. 2011, Gonzalez, Pham et al. 2014, Bahring, Dannenberg et al. 2001, Scannevin, Wang et al. 2004, Niwa, Nerbonne 2010). Although binding of DREAM to $\mathrm{Kv}$ channels is $\mathrm{Ca}^{2+}$ independent, the presence of $\mathrm{Ca}^{2+}$ alters the kinetics of $\mathrm{Kv}$ channel inactivation (Patel, Campbell et al. 2002). Crystallographic studies have shown that Kv4 interacts with KChIPs through its Nterminus of $\mathrm{T} 1$ domain and resulting $\mathrm{Kv} 4-\mathrm{KChIPs}$ complex is octamer (4:4 manner) in which each KChIPs molecule simultaneously interacts with two Kv4.3 molecules (Pioletti, Findeisen et al. 2006, Wang, Yan et al. 2007a). Two binding sites are involved in the formation of Kv4-KChIPs octamer. Site- 1 of Kv4 channel is composed of non-polar residues 2-22 and it binds to the deep hydrophobic pocket on the surface of KChIPs, whereas site-2 (residue 70-90) of Kv4 channel is composed of predominately polar residues and interacts with N-terminal helix-2 of KChIPs through the salt bridge and hydrophobic interaction (Pioletti, Findeisen et al. 2006, Callsen, Isbrandt et al. 2005, Wang, Yan et al. 2007a). Previous research in our group has demonstrated that $\mathrm{Ca}^{2+}$ and $\mathrm{Pb}^{2+}$ modulate the interaction between DREAM and site-1 and site-2 (Azam, Miksovska 2018, Gonzalez, Pham et al. 2014). To investigate whether $\mathrm{Cd}^{2+}$ association with DREAM alters DREAM interactions with site- 1 and site- $2, \mathrm{Cd}^{2+}$ DREAM constructs were titrated into FITClabeled site- 1 and site- 2 and the change in FITC-labeled peptide anisotropy was monitored (Figure 4.11 and 4.12). Equilibrium dissociation constants were obtained by fitting the data to Equation 1 and the values are listed in Table 4.4. Titration results reveal that $\mathrm{Cd}^{2+}$ WTDREAM $(\Delta 64)$ has seven-fold higher affinity for site-1 


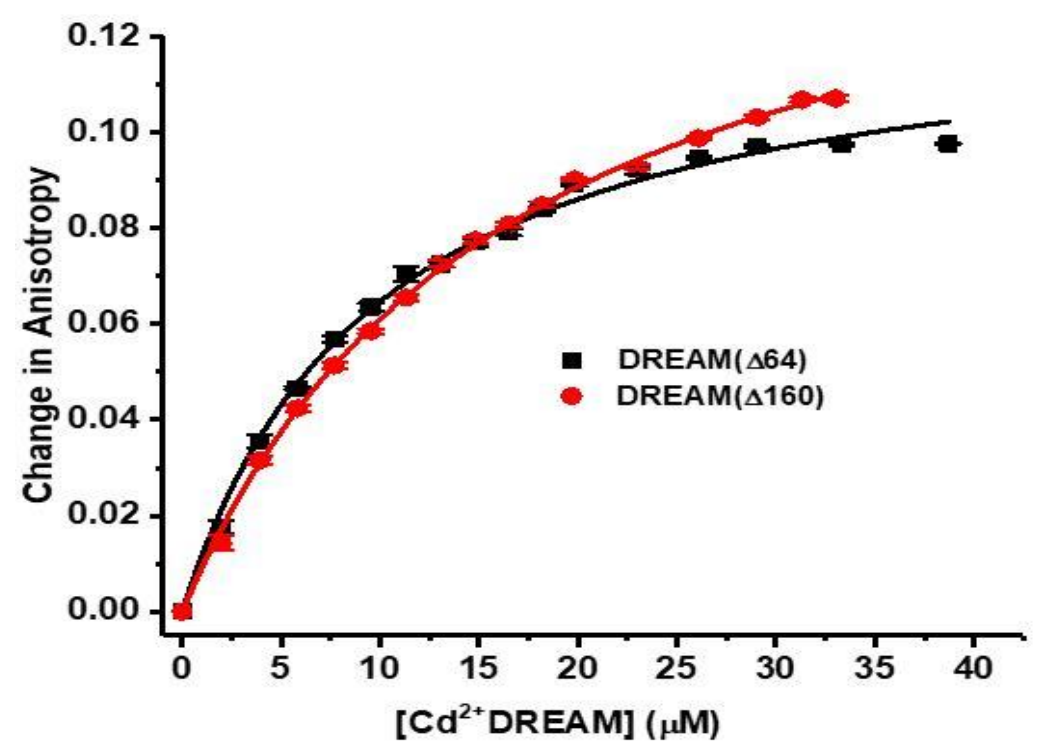

Figure 4.11. Titration of $0.5 \mu \mathrm{M}$ Kv4.3 (2-22) "site-1" against $\mathrm{Cd}^{2+}$-bound DREAM constructs. The solid lines represent the fits of the experimental data using Equation 1; standard

$\left(\mathrm{K}_{\mathrm{d}}=10 \pm 1 \mu \mathrm{M}\right)$ than $\left.\operatorname{apoDREAM}(\Delta 64)\left(\mathrm{K}_{\mathrm{d}}=70 \pm 1\right) \mu \mathrm{M}\right)$, and its affinity for site-1 is approximately four-times lower than that of $\mathrm{Ca}^{2+} \operatorname{WTDREAM}(\Delta 64)$ (Table 4.4). Considering that a hydrophobic site-1 binds to the hydrophobic cavity on DREAM surface, these results are consistent with WTDREAM( $\Delta 64): 1,8-\mathrm{ANS}$ emission data that demonstrate a lower exposure of hydrophobic surface for $\mathrm{Cd}^{2+}$ bound protein compared to $\mathrm{Ca}^{2+}$-bound protein that may result in a lower affinity for site-1 (Table 4.4). DREAM(E186) and DREAM(E234Q) did not show any binding to site-1 in metal-free form and in $\mathrm{Cd}^{2+}$-bound form. Inactivating $\mathrm{EF}-3$ in DREAM(E186Q) or EF-4 in DREAM(E234Q) might trigger structural reorganizations in the protein which disturb the hydrophobic cavity and consequently abolish binding of site-1. Site- 2 shows relatively weaker binding to $\mathrm{Cd}^{2+} \operatorname{DREAM}(\Delta 64)$ than $\mathrm{Ca}^{2+} \operatorname{WTDREAM}(\Delta 64)$, but the affinity of site-2 binding 


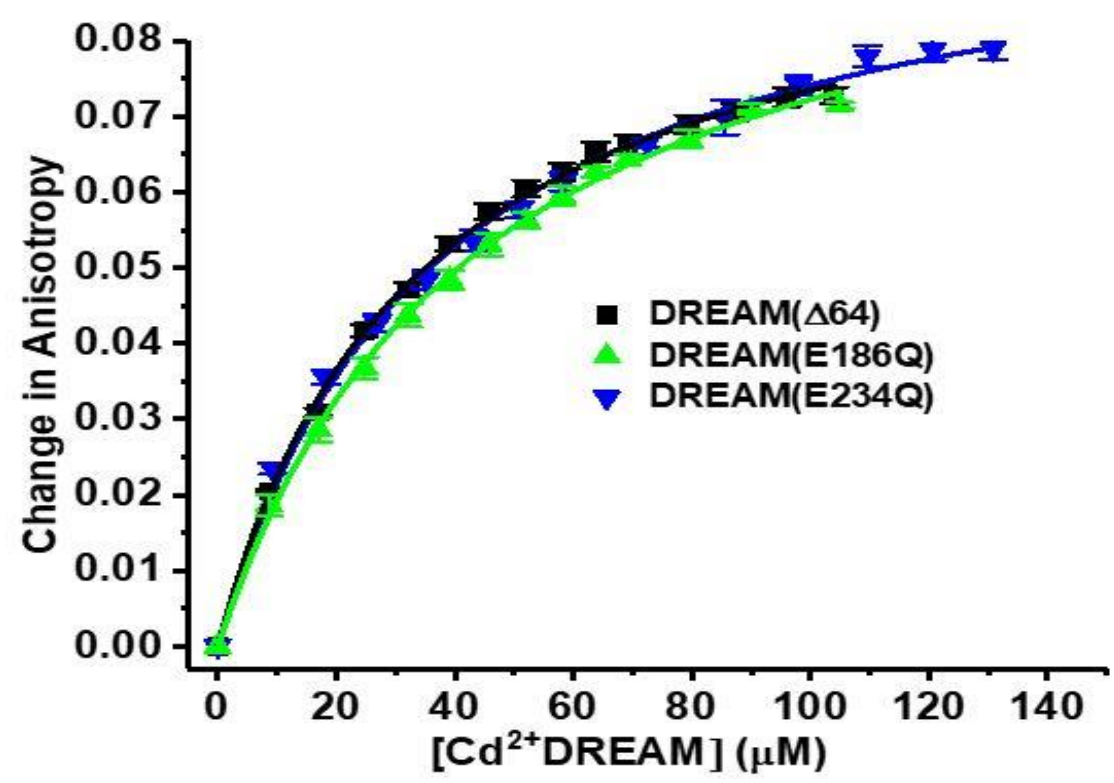

Figure 4.12. Titration of $0.5 \mu \mathrm{M} \mathrm{Kv} 4.3$ (70-90) "site-2" against $\mathrm{Cd}^{2+}$ bound DREAM constructs. The solid lines represent the fits of the experimental data using Equation 1; standard deviations are shown as error bars.

$\mathrm{Cd}^{2+} \mathrm{WTDREAM}(\Delta 64)$ is 15-times higher than site-2 binding to metal-free $\operatorname{DREAM}(\Delta 64)$, indicating $\mathrm{Cd}^{2+}$ binding may promote DREAM interactions with site-2 (Table 4.4). $\mathrm{Cd}^{2+}$ DREAM(E186) and $\mathrm{Cd}^{2+}$ DREAM(E234Q) bind to site-2 with an affinity comparable to site- 2 association with $\operatorname{Cd}^{2+} \operatorname{WTDREAM}(\Delta 64)$, suggesting binding of $\mathrm{Cd}^{2+}$ to a single EF-hand is sufficient to promote the site- 2 association to DREAM (Table 4.4). Taken together, the above results suggest that $\mathrm{Cd}^{2+}$ binding to DREAM may impact the deactivation kinetics of $\mathrm{Kv}$ channels complexes. Kv channel-KChIP complexes may represent a molecular target for $\mathrm{Cd}^{2+}$-induced memory and learning deficiencies as $\mathrm{Kv}$ channels have been 
implicated in hippocampus-dependent memory and learning (Lugo, Brewster et al. 2012). Considering high sequence homology of the neuronal calcium sensors (Ames, Lim 2012) and involvement of $\mathrm{Cd}^{2+}$ in different types of cancers (Waalkes 2000, Pesch, Haerting et al. 2000, Joseph, Muchnok et al. 2001) and neurodegenerative diseases, (Okuda, Iwamoto et al. 1997, Johnson 2001, Panayi, Spyrou et al. 2002) other members of NCS family could bind $\mathrm{Cd}^{2+}$ with high affinity and contribute to $\mathrm{Cd}^{2+}$-induced toxicity. 
Table 4.4. The equilibrium dissociation constants for PS1HL9, site-1 site-2 binding to DREAM constructs.

\begin{tabular}{|c|c|c|c|}
\hline DREAM constructs & PS1HL9 & Site-1 & Site-2 \\
\hline & $\mathrm{K}_{\mathrm{d}}(\mu \mathrm{M})$ & $\mathrm{K}_{\mathrm{d}}(\mu \mathrm{M})$ & $\mathrm{K}_{\mathrm{d}}(\mu \mathrm{M})$ \\
\hline \multicolumn{4}{|l|}{$\operatorname{DREAM}(\Delta 64)$} \\
\hline apo & $183 \pm 12$ & $70 \pm 3$ & -500 \\
\hline $\mathrm{Ca}^{2+}$ & $0.6 \pm 0.1$ & $2.7 \pm 0.1$ & $10 \pm 1$ \\
\hline $\mathrm{Cd}^{2+}$ & $6 \pm 1$ & $10 \pm 1$ & $34 \pm 1$ \\
\hline \multicolumn{4}{|l|}{$\operatorname{DREAM}(\Delta 160)$} \\
\hline apo & no binding & -450 & n.d. \\
\hline $\mathrm{Ca}^{2+}$ & $11 \pm 1$ & $24 \pm 1$ & n.d \\
\hline $\mathrm{Cd}^{2+}$ & $7 \pm 1$ & $16 \pm 1$ & n.d \\
\hline \multicolumn{4}{|l|}{ DREAM(E186Q) } \\
\hline apo & $149 \pm 22$ & no binding & n.d. \\
\hline $\mathrm{Ca}^{2+}$ & $32 \pm 1$ & no binding & n.d. \\
\hline $\mathrm{Cd}^{2+}$ & $16 \pm 1$ & no binding & $43 \pm 2$ \\
\hline \multicolumn{4}{|l|}{ DREAM(E234Q) } \\
\hline apo & $146 \pm 14$ & no binding & n.d. \\
\hline $\mathrm{Ca}^{2+}$ & $8 \pm 1$ & no binding & n.d. \\
\hline $\mathrm{Cd}^{2+}$ & $10 \pm 1$ & no binding & $36 \pm 2$ \\
\hline \multicolumn{4}{|c|}{$\begin{array}{l}\text { Data for DREAM }(\Delta 64) \text {, DREAM }(\Delta 160) \text {, DREAM(E186Q), and DREAM(E234Q) interactions } \\
\text { with PS1HL9 in apo and } \mathrm{Ca}^{2+} \text {-bound form are taken from reference (Pham, Miksovska 2016); data } \\
\text { for DREAM }(\Delta 64) \text { and DREAM }(\Delta 160) \text { interactions with site-1 and site-2 in apo and } \mathrm{Ca}^{2+} \text {-bound } \\
\text { form are taken from reference (Gonzalez, Pham et al. 2014). }\end{array}$} \\
\hline
\end{tabular}




\subsection{Summary}

In this article, using a combination of spectroscopic and calorimetric techniques we first time report $\mathrm{Cd}^{2+}$ binding to EF-hands in DREAM. We also show that $\mathrm{Cd}^{2+}$ association with DREAM modulates DREAM interactions with interacting partners, such as site- 1 and site- 2 of potassium voltage channel and presenilin-1. Interestingly, despite the fact that $\mathrm{Cd}^{2+}$ ion has similar ionic radii and charge/radius ratio values as $\mathrm{Ca}^{2+}$, the association of $\mathrm{Cd}^{2+}$ to EF-3 and EF-4 leads to a conformational transition that is somewhat restricted compared to that determined for the binding of the physiological ligand, $\mathrm{Ca}^{2+}$. This is evident by $1,8-\mathrm{ANS}$ emission data as well as by lower affinity constants for binding of peptides that mimic physiological effector proteins presenilin and $\mathrm{T} 1$ domain of $\mathrm{Kv}$ channels. However, the superior affinity of $\mathrm{Cd}^{2+}$ for DREAM suggest that under conditions of acute or chronic exposure to $\mathrm{Cd}^{2+}$, $\mathrm{Cd}^{2+}$ may bind to DREAM and probably other neuronal sensors and these interactions may provide insight into molecular basis of $\mathrm{Cd}^{2+}$ induced neurotoxicity. We hope this study will encourage future NMR and X-ray crystallography on $\mathrm{Cd}^{2+}$ bound neuronal calcium sensors to provide atomic-level insight into the mechanism of this neurotoxic metal's interactions with neuronal calcium sensors. 


\section{BINDING OF THE NEUROPROTECTIVE AGENT LITHIUM TO NEURONAL CALCIUM SENSOR PROTEIN DREAM: A NEW INSIGHT INTO LITHIUM THERAPY.}

\subsection{Background and significance}

$\mathrm{Li}^{+}$-based compounds have been used extensively for the treatment of severe mental disorders for more than six decades (Cade 1949, Marmol 2008, Vosahlikova, Svoboda 2016). However, the molecular mechanism of how lithium works remain one of the greatest challenges in modern psychology. Recent in vivo and in vitro studies in humans and rodents demonstrated the neuroprotective role of $\mathrm{Li}^{+}$and proposed its probable application for treatment of neurogenerative diseases such as Parkinson's disease, Alzheimer's disease (AD), and Huntington's disease (De Ferrari, Chacon et al. 2003, Youdim, Arraf 2004, Wei, Qin et al. 2001, Senatorov, Ren et al. 2004, Forlenza, De-Paula, Vanessa de Jesus Rodrigues et al. 2014). Despite numerous clinical applications of $\mathrm{Li}+$ in psychopharmacology for treating devastating diseases, the exact mechanism through which $\mathrm{Li}^{+}$exerts its therapeutic effects has not been elucidated yet. Several mechanisms have been proposed and its molecular targets remain unknown. Inhibitions of inositol monophosphatase (IMPase) and glycogen synthase kinase-3 beta (GSK-3 $\beta$ ) are the most relevant mechanisms of action (Forlenza, De-Paula, Vanessa de Jesus Rodrigues et al. 2014, Williams, Harwood 2000, Brown, Tracy 2013). IMPase is overexpressed in bipolar disorder patients and this overexpression causes inositol level increase (Dudev, Mazmanian et al. 2018, Haimovich, Eliav et al. 2012). According to the inositol depletion hypothesis, $\mathrm{Li}^{+}$treatment inhibits IMPase by displacing native cofactor $\mathrm{Mg}^{2+}$ and eventually decreases the level of inositol 
(Harwood 2005, Hallcher, Sherman 1980). Experimental studies in transgenic mice models overexpressing GSK-3 $\beta$ and in other animal models of Alzheimer's disease (AD) have demonstrated that chronic $\mathrm{Li}^{+}$administration causes a remarkable decrease in tau phosphorylation (Engel, Goñi-Oliver et al. 2006, Leroy, Ando et al. 2010, Noble, Planel et al. 2005). Similarly, it has been shown that chronic lithium administration reduces $A \beta_{42}$ (overproduction of this peptide has been associated with $\mathrm{AD}$ ) production through inhibition of GSK-3 $\beta$ and through modulation of the amyloid precursor protein (APP) (Rockenstein, Torrance et al. 2007, Su, Ryder et al. 2004). Multiple lines of studies have demonstrated that chronic $\mathrm{Li}^{+}$administration prevents or reverses neuronal cell death and thus $\mathrm{Li}^{+}$can act as a neuroprotective agent by up-regulating cell survival molecules, including brainderived neurotrophic factor (BDNF), cyclic AMP-responsive element binding protein (CREB), $\beta$-catenin, and brain grey matter volume, but by down-regulating pro-apoptotic activities, such as excitotoxicity, Bax, caspase, cytochrome c release (Wada, Yokoo et al. 2005).

Increased expression of neuronal calcium sensor protein-1 (NCS-1) and reduced gamma band activity has been reported in the brain of bipolar disorder patient (Koh, Undie et al. 2003, Özerdem, Güntekin et al. 2011). NCS-1 overexpression is considered responsible for the decrease in gamma-band activity (D'Onofrio, Kezunovic et al. 2015). $\mathrm{Li}^{+}$treatment has been shown to treat mood disturbances seen in bipolar disorder patients by inhibiting the interaction between NCS-1 and inositol 1,4,5-triphosphate receptor protein (Schlecker, Boehmerle et al. 2006a). Stasia D'Onofrio et al. (D'Onofrio, Hyde et al. 2017) have demonstrated that lithium treatment reduces the effects of overexpression of NCS-1 and thereby prevents the decrease of gamma-band activity, eventually restoring 
the normal level of gamma oscillation. Lithium-pilocarpine administration has been shown to alter the expression of voltage-gated potassium channel $4.2(\mathrm{Kv} 4.2)$ and potassium channel-interacting protein (KChIP1) and to alter calcium homeostasis in rat hippocampus ( $\mathrm{Su}$, Cong et al. 2008). A recent computation study based on competition between $\mathrm{Na}^{+}$and $\mathrm{Li}^{+}$in various neurotransmitters and G-protein coupled receptors $\mathrm{Na}^{+}$binding sites has revealed that neutral protein ligands such as $\mathrm{Thr} / \mathrm{Ser}$ hydroxyl group preferentially bind $\mathrm{Na}^{+}$, whereas negatively charged ligands such as Asp/Glu carboxylate favor $\mathrm{Li}^{+}$(Dudev, Mazmanian et al. 2018).

Decades of research have shown that monovalent ion $\mathrm{K}^{+}$activates enzymes including pyruvate kinase, tryptophanase, tyrosinase, and $\mathrm{Na}^{+}$activates thrombin, $\beta$ galactosidase, and tryptophan synthase (Page, Di Cera 2006, Gohara, Di Cera 2016). The highest affinity for $\mathrm{K}^{+}$-protein interaction reported to be $0.08-0.6 \mathrm{mM}$ range (Gohara, Di Cera 2016). It has been shown that $\mathrm{Na}^{+}$binds to thrombin with $\mathrm{K}_{\mathrm{d}} 110 \mathrm{mM}$ and $\mathrm{Na}^{+}$-bound form of thrombin is responsible for the blood procoagulant function of the enzyme (Page, Di Cera 2006). $\mathrm{Na}^{+}$also can occupy the EF-hand of parvalbumin and attenuates affinity of $\mathrm{Ca}^{2+}$ for parvalbumin (Henzl, Larson et al. 2004a). $\mathrm{Li}^{+}$shares more similarity with $\mathrm{Ca}^{2+}$ than $\mathrm{Na}^{+}$and $\mathrm{K}^{+}$, with charge/ionic radii ratio $1.47,2.02,1.03,0.75 \mathrm{e} / \AA ̊$, respectively, suggesting $\mathrm{Li}^{+}$has more likelihood to occupy $\mathrm{Ca}^{2+}$ binding site than $\mathrm{Na}^{+}$and $\mathrm{K}^{+}$(Page, Di Cera 2006).

Downstream regulatory element antagonist modulator (DREAM), also known as calsenilin, or potassium channel-interacting protein 3 (KChIP3), is a $29 \mathrm{kDa}$ neuronal calcium sensor (NCS) protein, which is expressed in the hippocampus of the brain (Carrion, Link et al. 1999). DREAM is a multifunction protein that has been linked to pain sensing, 
memory and learning process, $\mathrm{AD}$, and modulation of Kv4 expression and kinetics (An, Bowlby et al. 2000, Buxbaum, Choi et al. 1998, Cheng, Pitcher et al. 2002, Costigan, Woolf 2002, Fontán-Lozano, Romero-Granados et al. 2009, Jo, Jang et al. 2005, Abd Rashid, Hapidin et al. 2017). DREAM contains two domains, with each domain composed of two EF-hands. Nonfunctional EF-1 (residue 90-119) and EF-2 (residue 128-157) together with a short $\mathrm{N}$ - terminus $\alpha$-helix form an $\mathrm{N}$-terminal domain, whereas calcium-binding EF-3 (residue 163-192) and EF-4 (residue 211-240) along with a 13-residue long C-terminal $\alpha$ helix form a C-terminal domain (Lusin, Vanarotti et al. 2008). Among four EF-hands of DREAM, EF-1 is non-functional due to the presence of Cys 104 and Pro 105 at the EFhand loop, which distorts $\mathrm{Ca}^{2+}$-binding geometry; $\mathrm{EF}-2$ selectively binds $\mathrm{Mg}^{2+}$ because of the presence of Asp at the 12th position of the EF-loop rather than usual glutamate; and the canonical EF-3 and EF-4 preferentially bind $\mathrm{Ca}^{2+}$ with a $\mathrm{K}_{\mathrm{d}} 1-10 \mu \mathrm{M}$ (Gifford, Walsh et al. 2007, Osawa, Dace et al. 2005, Osawa, Tong et al. 2001). We have recently reported that EF-hands in DREAM are not calcium specific but can also bind lanthanide $\mathrm{Tb}^{3+}$ and toxic metal $\mathrm{Pb}^{2+}$ with an affinity that is superior to affinity for $\mathrm{Ca}^{2+}$ (Azam, Miksovska 2018, Gonzalez, Ramos et al. 2016). Based on the rationale of $\mathrm{Li}^{+}$induced alteration of expression of $\mathrm{KChIP1}$ (a protein homologous to DREAM), propensity of $\mathrm{Li}^{+}$binding through negatively charged amino acid residues, impact of $\mathrm{Li}^{+}$on hippocampus, and preponderance of negatively charged amino acid residues at EF-hand of DREAM, we postulate that DREAM is a molecular target of $\mathrm{Li}^{+}$. Our hypothesis is supported by our computation data which shows three potential lithium binding sites. In this Communication, using multiple lines of evidence, we first time demonstrate that $\mathrm{Li}^{+}$bind 
at EF-hand of DREAM; $\mathrm{Li}^{+}$binding leads to secondary and tertiary structural rearrangement of the protein; and $\mathrm{Li}^{+}$modulates DREAM interaction with $\mathrm{Kv} 4$ channel.

\subsection{Results and discussion}

To test the hypothesis that $\mathrm{Li}^{+}$binds to DREAM, our first approach was to probe the impact of $\mathrm{Li}^{+}$on emission spectra of $\operatorname{DREAM}(\Delta 64)$ construct that contains residues $65-$ 256. Emission spectra reveal that $\mathrm{Li}^{+}$addition to metal-free $\operatorname{DREAM}(\Delta 64)$ causes $-20 \%$ decrease in emission intensity, which is identical to a decrease observed for $\mathrm{Ca}^{2+}$ association to $\operatorname{DREAM}(\Delta 64)$, suggesting that both $\mathrm{Li}^{+}$and $\mathrm{Ca}^{2+}$ induce analogous
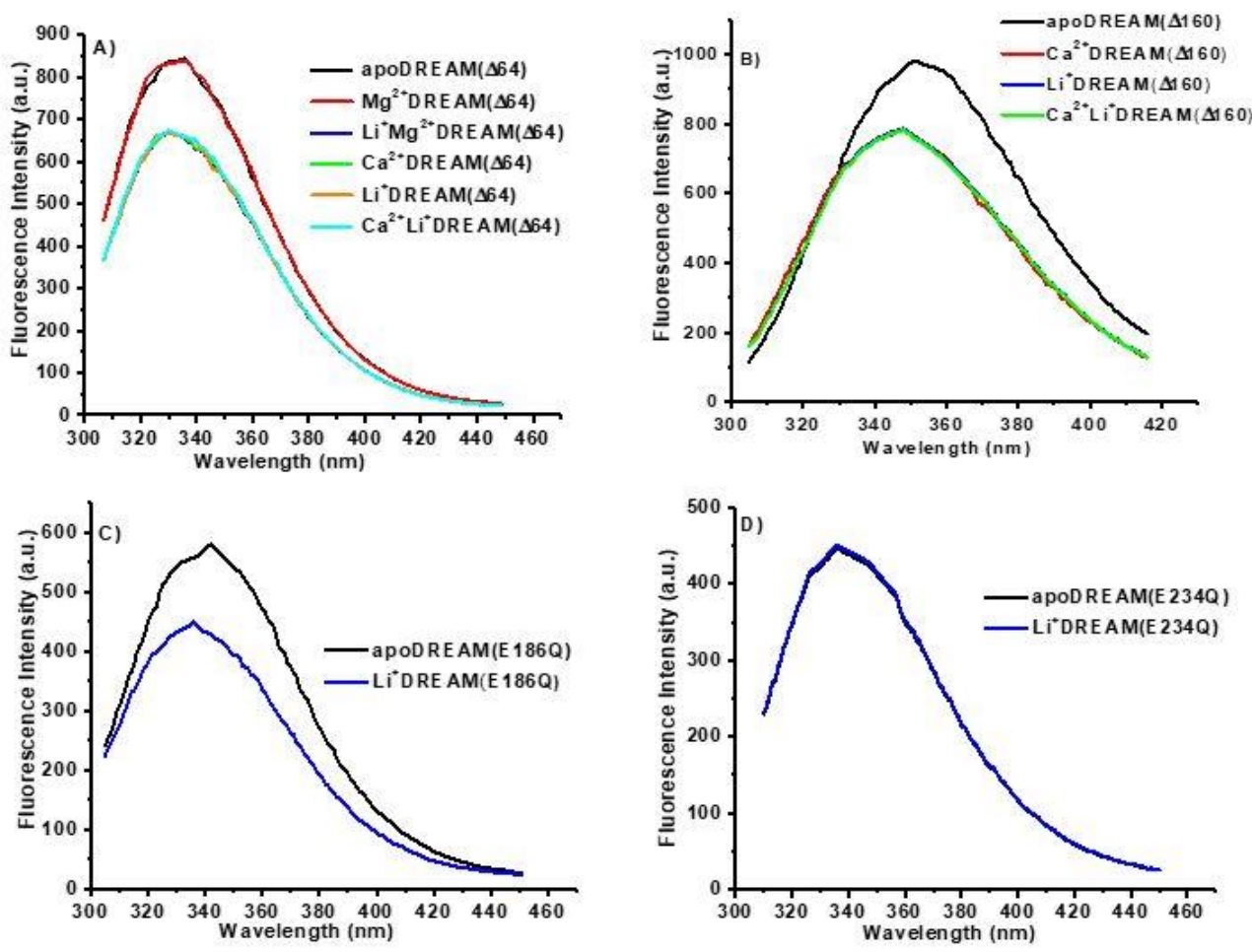

Figure 5.1. Impact of $\mathrm{Li}^{+}$binding on tryptophan emission of (A) DREAM $(\Delta 64)$, (B) DREAM( $\Delta 160)$, (C) DREAM(E186Q), and (D) DREAM(E234Q). In each case, $20 \mu \mathrm{M}$ protein samples in $20 \mathrm{mM}$ Tris were excited at $295 \mathrm{~nm}$; concentrations of EDTA, $\mathrm{Ca}^{2+}$, and $\mathrm{Li}^{+}$in each DREAM construct were $2 \mathrm{mM}$, $2 \mathrm{mM}$, and $50 \mu \mathrm{M}$, respectively; $\mathrm{Ca}^{2+} \mathrm{Li}+\mathrm{DREAM}$ constructs were prepared by adding $2 \mathrm{mM} \mathrm{Ca}^{2+}$ to DREAM constructs in the presence of $50 \mu \mathrm{M} \mathrm{Li}^{+}$. 
rearrangement in the protein tertiary structure resulting in the modification of the W169 surrounding (Figure 5.1A) and pointing towards the possibility that $\mathrm{Li}^{+}$may bind to the EF-hands. Also, no change in emission spectra was observed upon $\mathrm{Li}^{+}$addition to $\mathrm{Ca}^{2+} \operatorname{DREAM}(\Delta 64)$ and vice versa indicating that $\mathrm{Ca}^{2+}$ and $\mathrm{Li}^{+}$may bind to identical binding sites. The addition of $\mathrm{Li}^{+}$to $\mathrm{Mg}^{2+} \mathrm{DREAM}(\Delta 64)$ leads to a comparable decrease in emission intensity, suggesting that the occupation of EF-2 by $\mathrm{Mg}^{2+}$ does not impact $\mathrm{Li}^{+}$ interaction with DREAM. Analogous decrease in the emission spectra was observed upon $\mathrm{Li}^{+}$addition to the isolated C-terminal domain (DREAM( $\left.\Delta 160\right)$ ). In addition, the emission spectrum of DREAM $(\Delta 160)$ shifts from $351 \mathrm{~nm}$ in the absence of $\mathrm{Li}^{+}$to $348 \mathrm{~nm}$ in the presence of metal (Figure 5.1B) pointing towards the $\mathrm{Li}^{+}$binding site being located in the C-terminal domain. The longer wavelength emission maxima observed for $\operatorname{DREAM}(\Delta 160)$ is indicative of a more polar environment of W169 in this construct. To provide further insight into the location of $\mathrm{Li}^{+}$binding site, we measured emission spectra of DREAM(E186Q) and DREAM(E234Q) which have glutamate in the 12th position of the
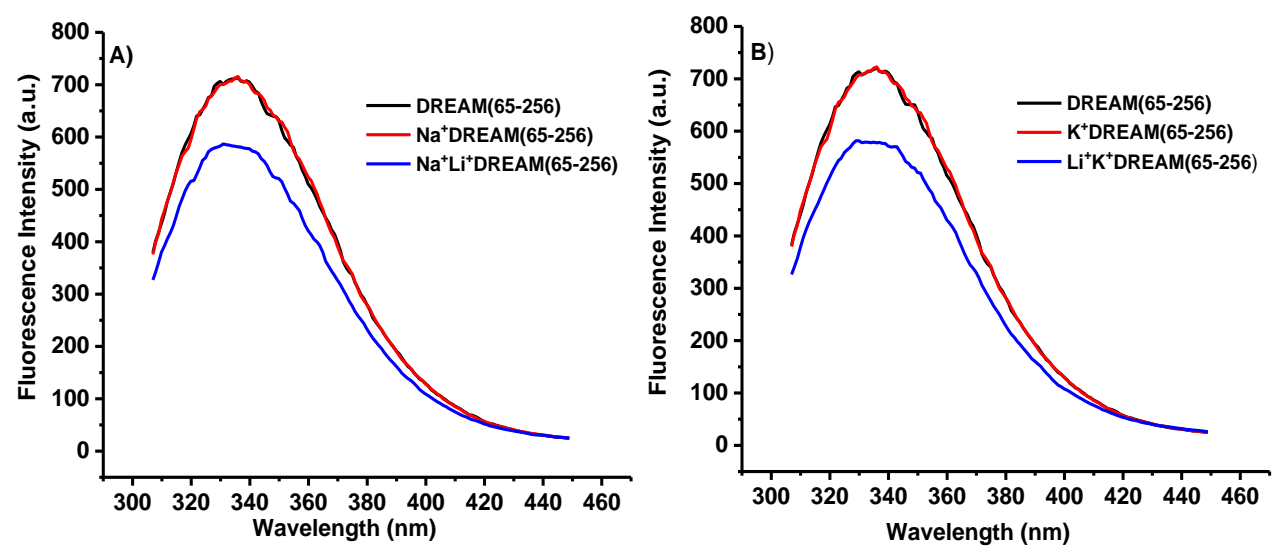

Figure 5.2. Impact of $\mathrm{Li}^{+}$binding to DREAM(65-256) in the presence of $100 \mathrm{mM}$ $\mathrm{Na}^{+}$or $100 \mathrm{mM} \mathrm{K}$. Condition: $20 \mu \mathrm{M}$ DREAM(65-256), $2 \mathrm{mM} \mathrm{Li}^{+}$, excited at 295 $\mathrm{nm}$. 
calcium-binding loop in EF-hand 3 or EF-hand 4, respectively, replaced by glutamine (Figure 5.1C,D). Emission spectra unveil that $\mathrm{Li}^{+}$or $\mathrm{Ca}^{2+}$ addition to DREAM(E186) results in a comparable decrease in emission intensity as observed for $\operatorname{DREAM}(\Delta 64)$, whereas emission spectra of $\mathrm{Li}^{+} \operatorname{DREAM}(\mathrm{E} 234 \mathrm{Q})$ and $\mathrm{Ca}^{2+} \mathrm{DREAM}(\mathrm{E} 234 \mathrm{Q})$ are superimposable to the spectrum for apoDREAM. This is consistent with the fact that $\mathrm{Li}^{+}$ or $\mathrm{Ca}^{2+}$ association to EF-4 triggers structural rearrangement which leads to a more efficient W169 emission quenching. The identical emission spectra for apoDREAM(E234Q) in the absence or presence of $\mathrm{Ca}^{2+}$ or $\mathrm{Li}^{+}$suggest an absence of
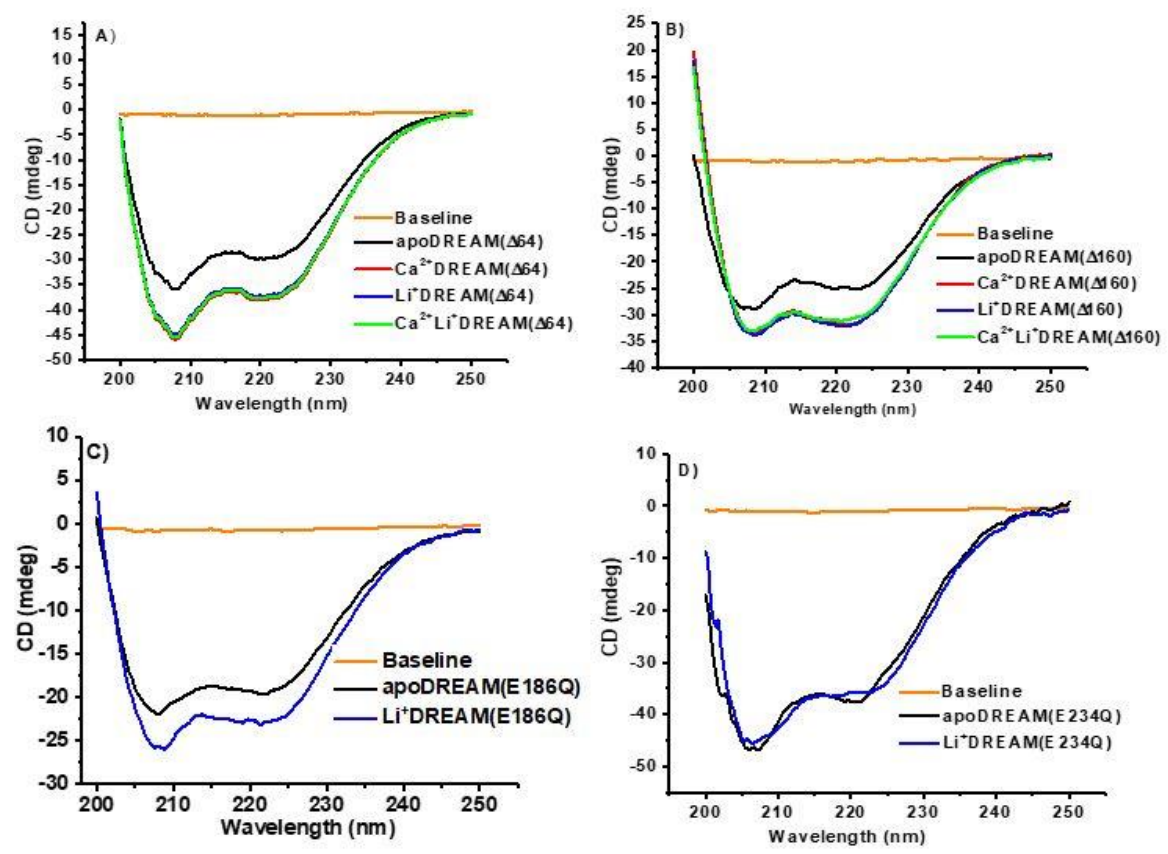

Figure 5.3. Impact of $\mathrm{Li}^{+}$binding on far-UV CD spectra of (A) $20 \mu \mathrm{M}$ $\operatorname{DREAM}(\Delta 64), \quad$ (B) $20 \quad \mu \mathrm{M} \quad \operatorname{DREAM}(\Delta 160), \quad$ (C) $20 \mu \mathrm{M}$ DREAM(E186Q), and (D) $20 \mu \mathrm{M}$ DREAM(E234Q) in $20 \mathrm{mM}$ Tris. Concentrations of EDTA, $\mathrm{Ca}^{2+}$, and $\mathrm{Li}^{+}$in each DREAM construct were $2 \mathrm{mM}, 2 \mathrm{mM}$ and $50 \mu \mathrm{M}$, respectively; $\mathrm{Ca}^{2+} \mathrm{Li}^{+} \mathrm{DREAM}$ constructs were obtained adding $2 \mathrm{mM} \mathrm{Ca}^{2+}$ to DREAM constructs in the presence of $50 \mu \mathrm{M} \mathrm{Li}^{+}$. All experiments were conducted at $18^{\circ} \mathrm{C}$ using a J-810 Jasco CD spectrometer through the $2 \mathrm{~mm}$ path of a $(2$ $\mathrm{mm} \times 10 \mathrm{~mm}$ ) quartz cuvette. 
conformational change affecting the W169 emission due to the metal binding to this EFhand. To eliminate non-specific interactions between $\mathrm{Li}^{+}$and DREAM, emission spectra were also recorded in the presence of $100 \mathrm{mM} \mathrm{NaCl}$ or $100 \mathrm{mM} \mathrm{KCl}$ (Figure 5.2). The addition of $\mathrm{Li}^{+}$to DREAM in the presence of monovalent salts leads to the analogous emission decrease, eliminating the contribution of the non-specific $\mathrm{Li}^{+}$binding to DREAM.

To gain insight into the impact of $\mathrm{Li}^{+}$binding on protein secondary structure, we measured the far-UV CD spectra of DREAM constructs (Figure 5.3 A-D). CD spectra unveil that ellipticity decreases analogously upon $\mathrm{Li}^{+}$and $\mathrm{Ca}^{2+}$ binding to DREAM constructs. The decrease in ellipticity could be attributed to an increase in $\alpha$-helices,

A)

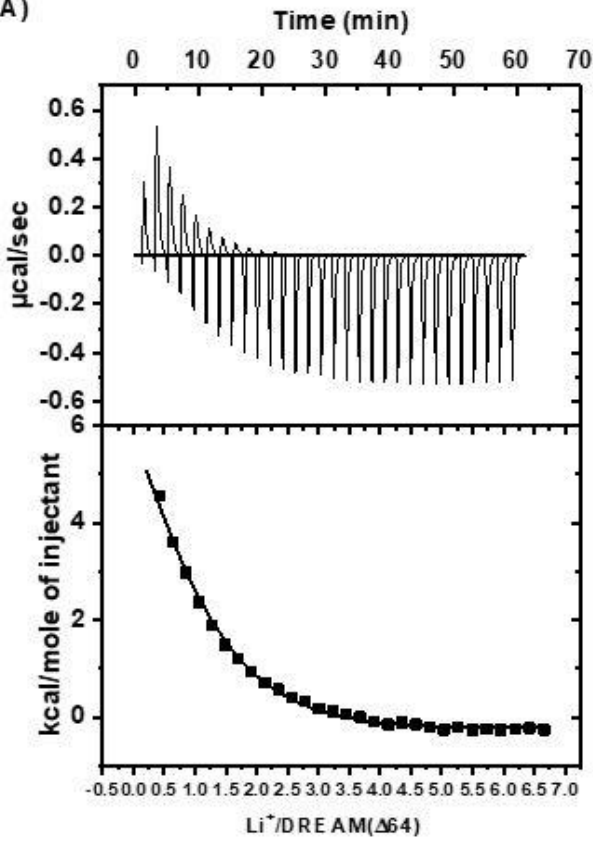

B)

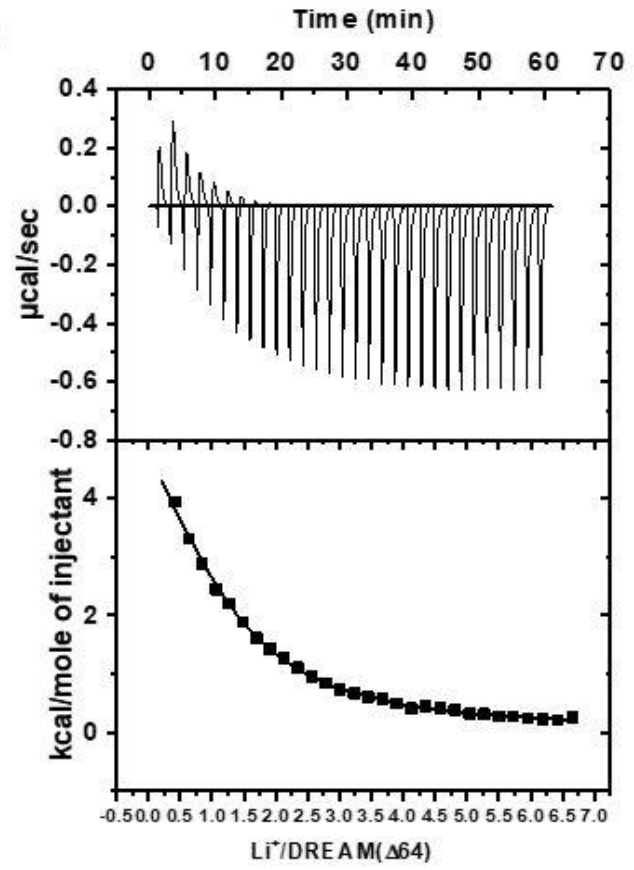

Figure 5.4. ITC isotherms for $\mathrm{Li}^{+}$binding to EF-hand of metal-free A) $\operatorname{DREAM}(\Delta 64)$ and B) DREAM $(\Delta 160) .30 \times 6 \mu \mathrm{L}$ of $1 \mathrm{mM} \mathrm{Li}^{+}$were titrated into 20 $\mu \mathrm{M}$ DREAM.Contribution of buffer was corrected. Thermal power expressed in $\mu \mathrm{cal} . \mathrm{s}^{-1}$ is shown in the upper panels of the profiles, whereas integrated reaction heats $(\Delta \mathrm{H})$ expressed in kcal.mole ${ }^{-1}$ is shown in the lower panels. The solid lines correspond to the best fitting curve using sequential model with two sites; thermodynamics parameters obtained from the model are listed in Table 1. 
rearrangement of $\alpha$-helices, or both. Similar CD spectra of $\mathrm{Li}^{+}$DREAM and $\mathrm{Ca}^{2+}$ DREAM can be interpreted bythe fact that $\mathrm{Li}^{+}$association triggers secondary structural rearrangement analogous to $\mathrm{Ca}^{2+}$ association.

Thermodynamic parameters for $\mathrm{Li}^{+}$binding to apoDREAM $(\Delta 64)$ were characterized in ITC study (Figure 5.4A and Table 5.1). Analysis of binding isotherms for $\mathrm{Li}^{+}$association to apoDREAM $(\Delta 64)$ using a two side sequential binding model reveals presence of two binding sites with association constants of $9.3 \pm 1.7 \times 10^{4} \mathrm{M}-1$ and $9 \pm 1$ $\mathrm{x} 10^{3} \mathrm{M}^{-1}$ and comparable association constants were measured for $\mathrm{Li}^{+}$binding to apoDREAM $(\Delta 160)$ (Figure 5.4B and Table 5.1). $\mathrm{Li}^{+}$binding to the higher affinity site is an endothermic reaction with a negligible change in enthalpy whereas association to the lower affinity site displays a positive enthalpy and entropy change. The apparent equilibrium dissociation constant $\left(\mathrm{K}_{\mathrm{d}}\right)$ for $\mathrm{Li}^{+}$association to metal-free $\operatorname{DREAM}(\Delta 64)$ and
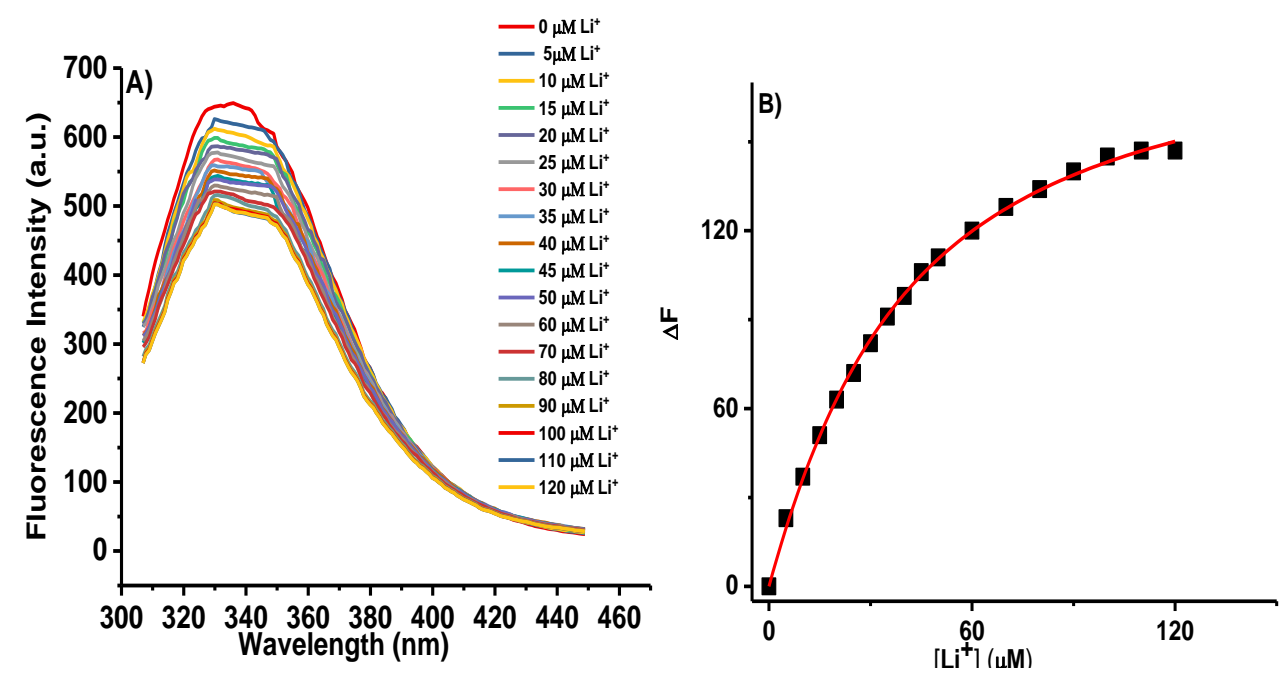

Figure 5.5. A) Emission intensity of $20 \mu \mathrm{M}$ metal-free $\operatorname{DREAM}(\Delta 64)$ upon $\mathrm{Li}^{+}$ addition; DREAM $(\Delta 64)$ was prepared in $20 \mathrm{mM}$ Tris, excited at $295 \mathrm{~nm}$ B) Titration curve for $\mathrm{Li}+$ binding to metal-free $\operatorname{DREAM}(\Delta 64) . \Delta \mathrm{F}$ was calculated based on decrease in Trp169 emission intensity at emission maximum. Solid line represents fit of the data using Equation 2. 
$\operatorname{DREAM}(\Delta 160)$ is $34 \pm 4 \mu \mathrm{M}$ and $50 \pm 4 \mu \mathrm{M}$, respectively, indicating one order of magnitude lower affinity of $\mathrm{EF}-$ hands for $\mathrm{Li}^{+}$than for its physiological ligand calcium (Equation 1). The analogous $K_{d}$ value was obtained by observing changes in the Trp emission upon $\mathrm{Li}^{+}$titration into apoDREAM sample (Figure 5.5). The titration curve was analyzed using a single binding site model, (Equation 2) and provided an equilibrium dissociation constant of $31 \pm 5 \mu \mathrm{M}$ in a good agreement with the ITC results. We have also measured binding isotherms for $\mathrm{Li}^{+}$association to DREAM in the presence of $100 \mu \mathrm{M}$ $\mathrm{Ca}^{2+}$. The titration resulted in a negligible signal confirming that $\mathrm{Li}^{+}$does not associate to $\mathrm{Ca}^{2+}$-bound DREAM either due to the occupation of binding sites by $\mathrm{Ca}^{2+}$ or $\mathrm{Li}^{+}$binding sites does not overlay with binding sites for $\mathrm{Ca}^{2+}$, but are inaccessible in $\mathrm{Ca}^{2+}$ bound DREAM. 
Table 5.1. Thermodynamics parameters recovered for $\mathrm{Li}^{+}$binding to EF-hand of metal-free DREAM.

\begin{tabular}{|c|c|c|c|c|c|c|}
\hline Samples & $\begin{array}{l}\mathrm{K}_{1} \quad \mathrm{X} \quad 10^{4} \\
\left(\mathrm{M}^{-1}\right)\end{array}$ & $\begin{array}{l}\mathrm{K}_{2} \quad \mathrm{X} \\
\left(\mathrm{M}^{-1}\right)\end{array}$ & $\begin{array}{l}\Delta \mathrm{H}_{1} \\
(\mathrm{kcal} / \mathrm{mole})\end{array}$ & $\begin{array}{c}\Delta \mathrm{H}_{2} \\
(\mathrm{kcal} / \mathrm{mole})\end{array}$ & $\begin{array}{l}\mathrm{T} \Delta \mathrm{S}_{1} \\
\text { (kcal/mole) }\end{array}$ & $\begin{array}{l}\mathrm{T} \Delta \mathrm{S}_{2} \\
(\mathrm{kcal} / \mathrm{mole})\end{array}$ \\
\hline $\operatorname{DREAM}(\Delta 64)+\mathrm{Li}^{+}$ & $9.3 \pm 1.7$ & $0.9 \pm 0.1$ & $8.8 \pm 0.4$ & $-8.6 \pm 0.2$ & $15.5 \pm 0.3$ & $-3.2 \pm 0.3$ \\
\hline $\operatorname{DREAM}(\Delta 160)+\mathrm{Li}^{+}$ & $7.3 \pm 0.6$ & $0.6 \pm 0.1$ & $7.8 \pm 0.8$ & $3.3 \pm 0.8$ & $14.3 \pm 0.8$ & $8.0 \pm 1.1$ \\
\hline
\end{tabular}


The $200 \mathrm{~ns}$ molecular dynamic (MD) simulation of $\mathrm{Li}^{+}$DREAM complex structure revealed several $\mathrm{Li}^{+}$binding sites on the protein surface (Figure 5.6). $\mathrm{Li}^{+}$, as a hard atom, preferentially interacts with oxygen atoms. One $\mathrm{Li}^{+}$binding site on DREAM surface is formed by sidechain residues that coordinate $\mathrm{Ca}^{2+}$ in the EF-hand 4, namely Ap 223 and Glu 234. Another binding site is formed by side chains of residues Asp134 and Asp 141 that belongs to the $\mathrm{Mg}^{2+}$ binding EF-hand 2. $\mathrm{Li}^{+}$ions were also found to bind to other negatively charged residues, namely Asp242 and Glu243 at the C- terminus and Glu164 and Asp 134 at the N-terminal domain. However, these interactions are likely to be transient and unlikely to contribute to the observed changes in the DREAM secondary and tertiary structure. Interestingly, $\mathrm{Li}^{+}$association to the protein leads to the stabilization of the protein structure as evident from the decreased root mean square fluctuation of backbone atoms during the last $50 \mathrm{~ns}$ of the simulation with respect to the initial $20 \mathrm{~ns}$. Previous data showed that the lithium cation is coordinated with 4 to 6 oxygen atoms in complex with carboxylic acids (Olsher, Izatt et al. 1991). A survey of the protein data bank reveals 29 protein structures with bound $\mathrm{Li}^{+}$ion. In the majority of cases, $\mathrm{Li}^{+}$is coordinated to the protein through two to four oxygen atoms, predominantly from the side chains of aspartic acid residue and from carbonyl oxygen in agreement with the MD data presented here.

As calmodulin $(\mathrm{CaM})$ and parvalbumin were previously shown to bind monovalent cations $\mathrm{Na}^{+}$and $\mathrm{K}^{+}$albeit with a millimolar affinity (Henzl, Larson et al. 2004b), we tested if $\mathrm{Li}^{+}$associates to rat $\mathrm{CaM}$ and trigger changes in the protein secondary or tertiary structure. The $\mathrm{CD}$ spectrum of rat $\mathrm{CaM}$ in the presence of $\mathrm{Li}^{+}$overlays with that for apoCaM, whereas upon $\mathrm{Ca}^{2+}$ addition, the $\mathrm{CD}$ signal decreases which are consistent with $\mathrm{Ca}^{2+}$ binding to apoCaM suggesting that $\mathrm{Li}^{+}$does not bind to $\mathrm{CaM}$ or its association does 

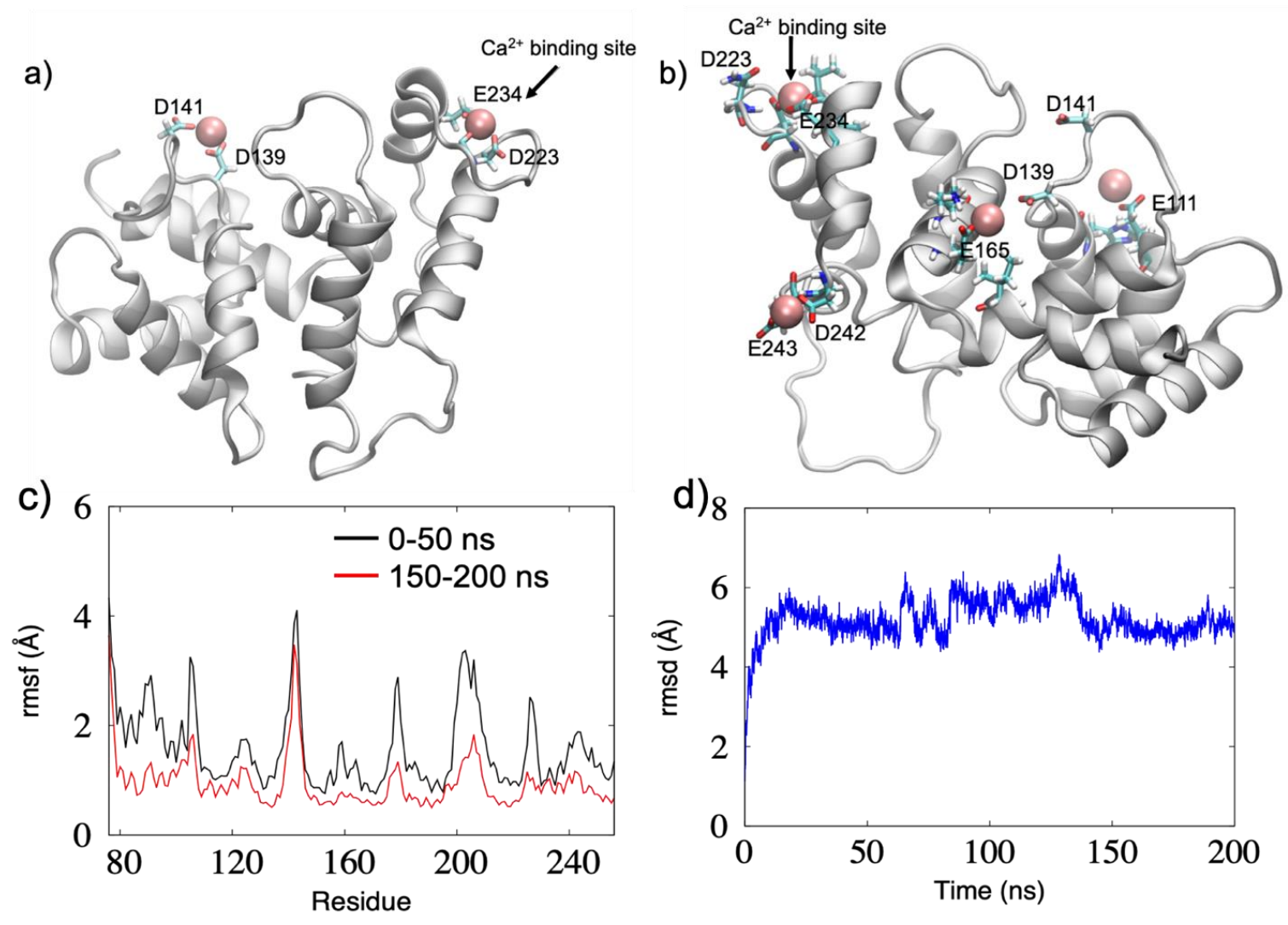

Figure 5.6. (a) Lithium ions strongly bound to DREAM protein as revealed by the molecular simulations. One of the binding sites (right) is also a calcium binding site. b) Other transient binding sites observed during the simulations. (c) Root mean square fluctuation of backbone atoms in protein for the first $50 \mathrm{~ns}$ (black) and the last $50 \mathrm{~ns}$ (red) of simulation. Protein conformational flexibility is significantly reduced upon biding with lithium ions. (d) Root mean square deviation of protein with respect to time, showing a stable protein- $\mathrm{Li}^{+}$complex.

not cause changes in the protein secondary structure (Figure 5.7A). The absence of Trp residue in CaM sequence allows for monitoring CaM structural change associated with metal binding to the $\mathrm{N}$ - terminal domain using intrinsic Phe emission and binding of metal to the C- terminal domain using intrinsic Tyr emission (VanScyoc, Sorensen et al. 2002). The emission spectrum of apoCaM using $250 \mathrm{~nm}$ excitation shows an emission maximum at $300 \mathrm{~nm}$ that corresponds to the emission of Phe residues located in the $\mathrm{N}$ - terminus ( Figure 5.7B). The emission intensity increases upon the addition of $2 \mathrm{mM} \mathrm{Ca}^{2+}$, but no 

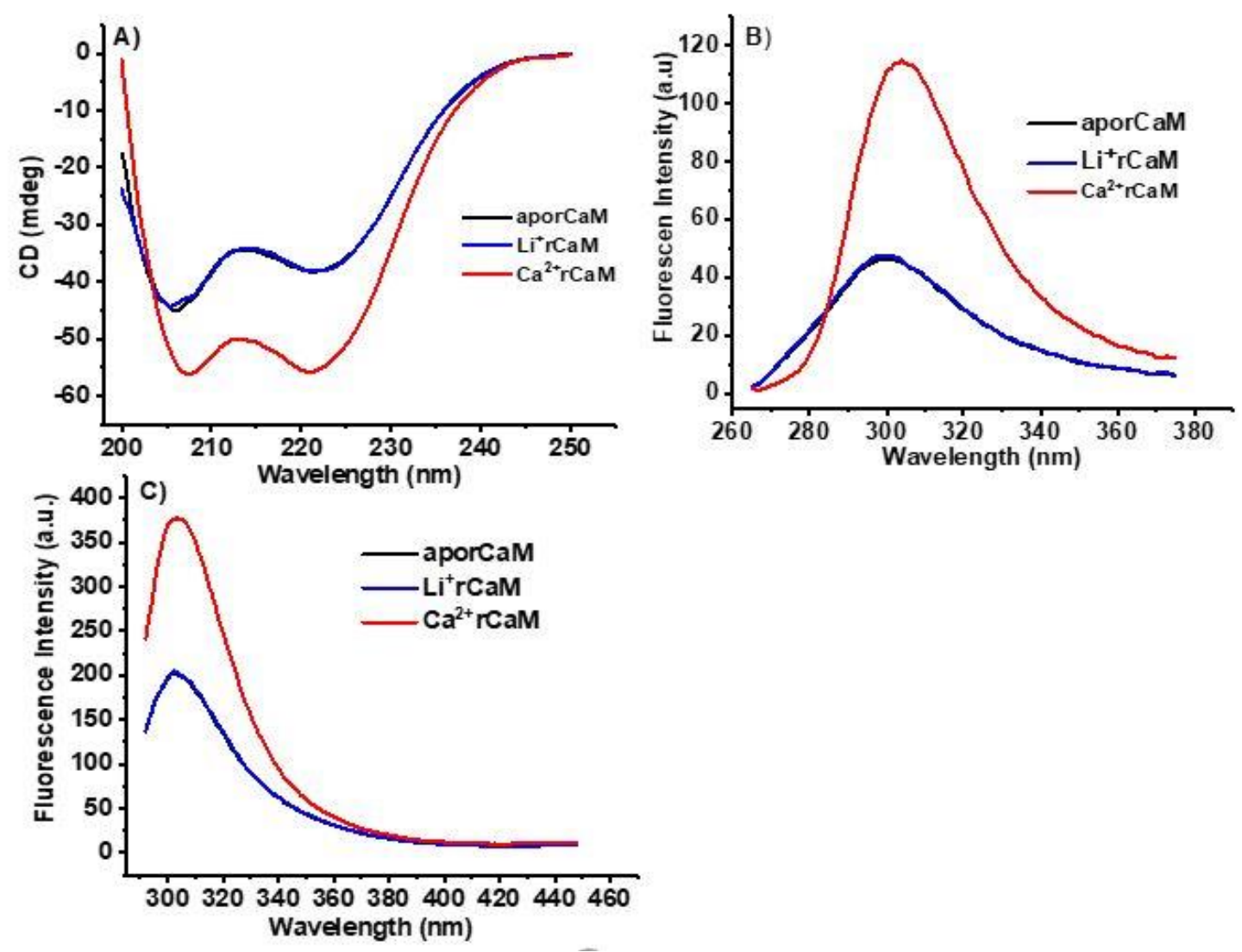

Figure 5.7. Impact of $\mathrm{Li}^{+}$binding on secondary structure of $20 \mu \mathrm{M} \mathrm{rCaM}$ in $20 \mathrm{mM}$ Tris (A). Consequence of $\mathrm{Li}^{+}$binding on the phenylalanine emission (B) and tyrosine emission (C) of the same protein in the same condition. Samples were excited at $250 \mathrm{~nm}$ and $277 \mathrm{~nm}$ for Figure (B) and (C), respectively. Concentrations of EDTA, $\mathrm{Ca}^{2+}$ and $\mathrm{Li}^{+}$ were $2 \mathrm{mM}, 2 \mathrm{mM}$, and $2 \mathrm{mM}$, respectively.

increase was observed upon the addition of $2 \mathrm{mM} \mathrm{Li}^{+}$. The $277 \mathrm{~nm}$ excitation leads to the emission spectrum with a maximum at $304 \mathrm{~nm}$ (Figure 5.7C). Addition of $2 \mathrm{mM} \mathrm{Li}^{+}$to apoCaM does not result in changes in the emission spectrum of Tyr residues located in the C- terminal domain whereas addition of $2 \mathrm{mM} \mathrm{Ca}^{2+}$ increases the emission intensity at 304 $\mathrm{nm}$ in agreement with $\mathrm{Ca}^{2+}$ binding to the EF-hand 3 and 4 in the $\mathrm{C}$ - terminal domain. These results suggest that $\mathrm{Li}^{+}$does not bind to EF-hand of CaM or its binding does not cause structural changes that are analogous to those triggered by $\mathrm{Ca}^{2+}$ association. Thus 
unlike CaM, the EF-hands in the DREAM are able to bind $\mathrm{Li}^{+}$at submillimolar concentration, suggesting that $\mathrm{Li}^{+}$binding to EF-hands may be specific for DREAM and possibly other NCS proteins. This is supported by a previous result that demonstrated a decrease in the Trp residue emission upon $\mathrm{Li}^{+}$binding to neuronal calcium sensor 1 (NCS1), although no further characterization of $\mathrm{Li}^{+}$interactions with isolated NCS-1 protein (Schlecker, Boehmerle et al. 2006b).

Previous studies from our group have shown that binding of $\mathrm{Ca}^{2+}$ or toxic metals such as $\mathrm{Cd}^{2+}$ or $\mathrm{Pb}^{2+}$ alters DREAM interaction with hydrophobic molecule 1,8-ANS (Azam, Miksovska 2018, Gonzalez, Miksovska 2014). 1,8-ANS has been widely used for assaying hydrophobicity of protein surface as emission intensity of 1,8-ANS increases upon binding to hydrophobic cavities on the protein surface. Utilizing AutoDock software version 4.2, we identified probable 1,8-ANS binding sites on the DREAM surface (Gonzalez, Miksovska 2014, Morris, Huey et al. 2009). One binding site was identified between then helix-8 and helix-9 of EF-4, while another site was found between EF-3 and EF-4. To investigate whether $\mathrm{Li}^{+}$association impacts DREAM affinity for 1,8-ANS, we measured emission spectra of DREAM-1,8-ANS in the presence of $\mathrm{Ca}^{2+}, \mathrm{Li}^{+}$, and in the metal-free form (Figure 5.8). Increase in emission intensity of 1,8-ANS upon binding to apoDREAM confirms 1,8-ANS binding to DREAM $(\Delta 64)$. Compared with apoDREAM( $(464)-\mathrm{ANS}$, higher emission intensity is observed for $\operatorname{Li}^{+} \operatorname{DREAM}(\Delta 64)$ ANS, suggesting $\mathrm{Li}^{+}$binding exposes the hydrophobic cavity on the DREAM surface, which facilitates 1,8-ANS binding. However, $\mathrm{Li}^{+} \operatorname{DREAM}(\Delta 64)$-ANS has a lower emission intensity than $\mathrm{Ca}^{2+}$ DREAM $(\Delta 64)-\mathrm{ANS}$, indicating $\mathrm{Ca}^{2+}$ association to DREAM exposes the hydrophobic cavity more than $\mathrm{Li}^{+}$association. DREAM $(\Delta 160)-\mathrm{ANS}$ 
complexes follow the same trend as $\operatorname{DREAM}(\Delta 64)$-ANS, providing additional evidence that $\mathrm{Li}^{+}$binds to the $\mathrm{C}$ - terminal domain and its interaction with protein exposes the hydrophobic cavity of DREAM. To corroborate the finding of emission study, we determined lifetimes of $\operatorname{DREAM}(\Delta 64)-$ ANS complexes utilizing frequency domain fluorometry (Figure 5.9 and Table 5.2). 1,8-ANS bound to metal-free DREAM shows two fluorescent lifetimes ( $\tau_{1}=4 \mathrm{~ns}$ and $\tau_{2}=16.8 \mathrm{~ns}$ ), which is consistent with 1,8-ANS binding to two distinct binding sites on DREAM surface (Table 5.2). $\mathrm{Li}^{+}$association with metalfree DREAM $(\Delta 64)$ :1,8-ANS leads to an increase in longer lifetime $\left(\tau_{2}=17.9 \mathrm{~ns}\right)$ and an increase in the pre-exponential factor $\alpha_{2}$ from $21.2 \%$ in metal-free $\operatorname{DREAM}(\Delta 64): 1,8$ ANS to $35.8 \%$ in $\operatorname{Li}^{+} \operatorname{DREAM}(\Delta 64): 1,8-\mathrm{ANS}$, bolstering the findings of emission study that $\mathrm{Li}^{+}$binding to DREAM induces structural reorganization in the protein that exposes the hydrophobic cavity. Additionally, $\mathrm{Ca}^{2+}$ association with metal-free $\operatorname{DREAM}(\Delta 64): 1,8$ -
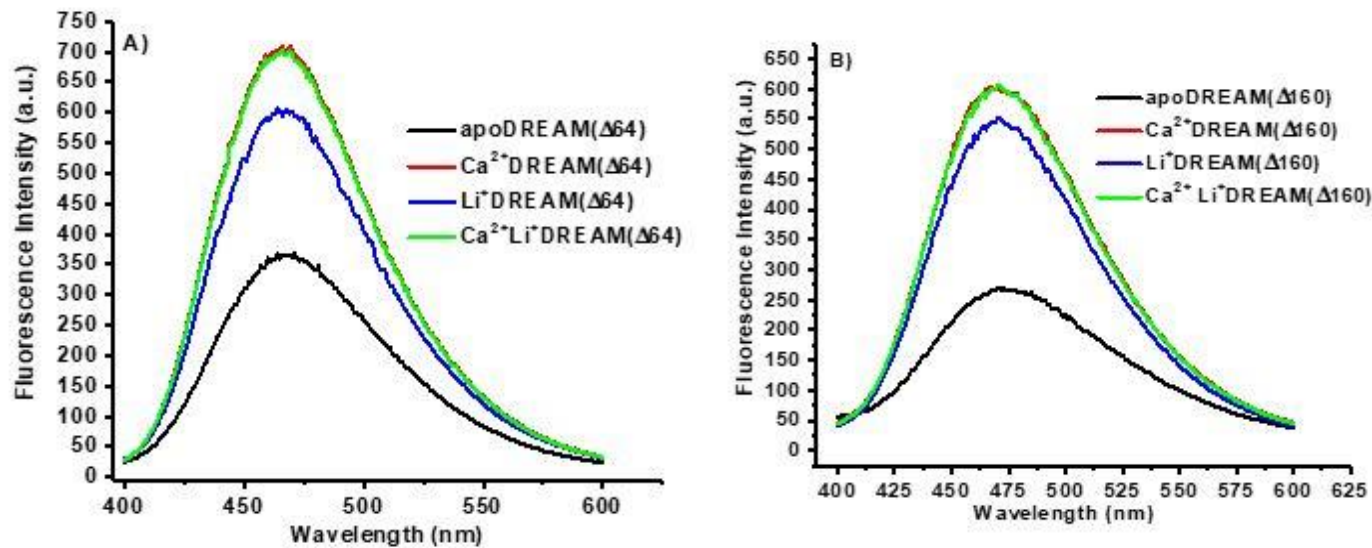

Figure 5.8. Consequences of $\mathrm{Li}^{+}$binding on emission spectra of (A) 1,8ANS:DREAM $(\Delta 64)$, (B) 1,8-ANS:DREAM $(\Delta 160$ complexes. Conditions: $20 \mu \mathrm{M}$ DREAM, $20 \mu \mathrm{M}$ 1,8-ANS; excited at $350 \mathrm{~nm}$. Concentrations of EDTA, $\mathrm{Ca}^{2+}$, and $\mathrm{Li}^{+}$ were same as tryptophan emission. 
ANS causes further increase in $\alpha_{2}$, which is consistent with additional changes in the protein structure.

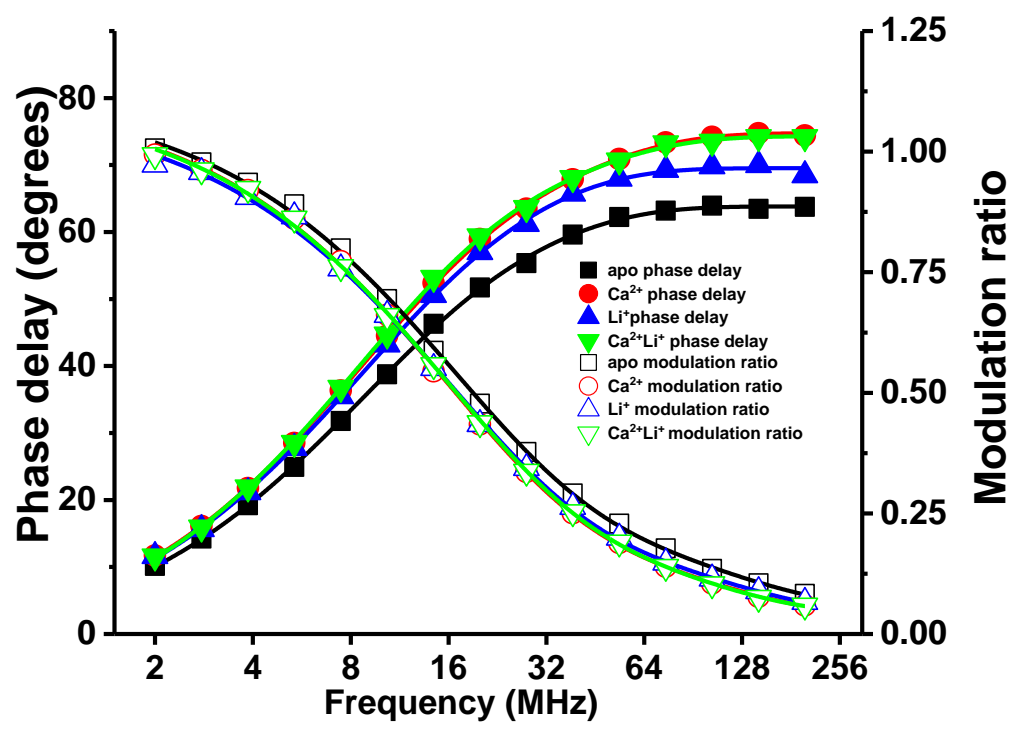

Figure 5.9. Frequency-domain intensity decay of $40 \mu \mathrm{M}$ 1,8-ANS:54 $\mu \mathrm{M}$ $\operatorname{DREAM}(\Delta 64)$ complexes in $20 \mathrm{mM}$ Tris and in the presence of $2 \mathrm{mM}$ EDTA (squares), $2 \mathrm{mM} \mathrm{Ca}^{2+}$ (circle), $50 \mu \mathrm{M} \mathrm{Li}^{+}$(up triangle), and $\left(2 \mathrm{mM} \mathrm{Ca}^{2+}\right.$ and $50 \mu \mathrm{M} \mathrm{Li}^{+}$) $\mathrm{Ca}^{2+} \mathrm{Li}^{+}$(down triangle). Phase delay and modulation ratio are respectively shown as solid and empty symbols. Solid lines correspond to the fitting of the data using three-exponential decay model. 
Table 5.2. Fluorescence decay parameters for $\mathrm{Li}^{+}$and/or $\mathrm{Ca}^{2+}$ binding to DREAM $(\Delta 64)-1,8$-ANS complexes.

\begin{tabular}{lllllllll}
\hline Samples & $\tau_{1}(\mathrm{~ns})$ & $\tau_{2}(\mathrm{~ns})$ & $\alpha_{0}(\%)$ & $\alpha_{1}(\%)$ & $\alpha_{2}(\%)$ & $\mathrm{f}_{1}(\%)$ & $\mathrm{f}_{2}(\%)$ & $\chi^{2}$ \\
\hline apoDREAM $(\Delta 64)$ & $4.0 \pm 0.1$ & $16.8 \pm 0.2$ & 62.4 & 15.4 & 21.2 & $15.1 \pm 0.9$ & $83.2 \pm 1.0$ & 1.0 \\
\hline $\mathrm{Ca}^{2+} \mathrm{DREAM}(\Delta 64)$ & $4.2 \pm 0.2$ & $18.1 \pm 0.1$ & 41.2 & 16.5 & 42.3 & $9.8 \pm 0.6$ & $89.1 \pm 0.5$ & 0.9 \\
\hline $\mathrm{Li}^{+} \mathrm{DREAM}(\Delta 64)$ & $4.1 \pm 0.2$ & $17.9 \pm 0.2$ & 48.6 & 15.6 & 35.8 & $9.6 \pm 0.5$ & $89.2 \pm 0.5$ & 0.9 \\
\hline $\mathrm{Ca}^{2+} \mathrm{Li}^{+} \mathrm{DREAM}(\Delta 64)$ & $4.2 \pm 0.2$ & $18.1 \pm 0.1$ & 41.0 & 16.8 & 42.2 & $9.7 \pm 0.5$ & $89.1 \pm 0.5$ & 0.9
\end{tabular}

Data obtained from Vinci analysis software using a sum of three discrete exponential decays with fixed 1,8-ANS lifetime $\left(\tau_{0}=0.28 \mathrm{~ns}\right) . \tau_{0}, \tau_{1}$, and $\tau_{2}$ represent lifetimes of the three discrete exponential decays; $\mathrm{f}_{0}, \mathrm{f}_{1}$ and $\mathrm{f}_{2}$ are exponential decay fractions; $\alpha_{0}, \alpha_{1}$, and $\alpha_{2}$ represent pre-exponential factors. $\chi^{2}$ represents the goodness of the fit. Phase and modulation errors were respectively set at $\leq 0.2^{\circ}$ and $\leq 0.004$. 
It has been shown previously that $\mathrm{Ca}^{2+}$ modulates DREAM interactions with $\mathrm{Kv} 4$ channels and presenilin-1 (An, Bowlby et al. 2000, Jo, Jang et al. 2005, Gonzalez, Pham et al. 2014, Pham, Miksovska 2016). Recent studies from our group have shown that $\mathrm{Pb}^{2+}$ and $\mathrm{Cd}^{2+}$ also modulate the aforementioned interactions (Azam, Miksovska 2018). To investigate whether $\mathrm{Li}^{+}$association to the protein also alters DREAM interactions with Kv4 channel, we measured changes in the anisotropy of FITC-labeled peptides that correspond to residues 2-22 and 70-90 of Kv4.3 upon addition $100 \mu \mathrm{M} \operatorname{DREAM}(\Delta 64)$ and $200 \mu \mathrm{M}$ $\operatorname{DREAM}(\Delta 64)$, respectively (Figure 5.10A,B). Anisotropy of Kv4.3(2-22) increases from $0.063 \pm 0.008$ to $0.099 \pm 0.007,0.229 \pm 0.014$, and $0.218 \pm 0.010$ upon addition of metalfree $\operatorname{DREAM}(\Delta 64), \mathrm{Ca}^{2+} \operatorname{DREAM}(\Delta 64)$, and $\operatorname{Li}^{+} \operatorname{DREAM}(\Delta 64)$, respectively, and anisotropy of $\mathrm{Kv} 4.3(70-90)$ increases from $0.074 \pm 0.005$ to $0.116 \pm 0.004,0.193 \pm 0.012$, and $0.186 \pm 0.009$ upon addition of metal-free $\operatorname{DREAM}(\Delta 64), \mathrm{Ca}^{2+} \operatorname{DREAM}(\Delta 64)$, and $\mathrm{Li}^{+} \operatorname{DREAM}(\Delta 64)$, respectively. Significantly higher anisotropy of $\mathrm{Kv} 4.3(2-22)-\mathrm{Li}^{+}$ $\operatorname{DREAM}(\Delta 64)$ and $\mathrm{Kv} 4.3(70-90)-\mathrm{Li}^{+} \operatorname{DREAM}(\Delta 64)$ compared with the corresponding apo-form indicates an increased affinity of Li+DREAM for Kv4.3(2-22) and Kv4.3(7090. Similar anisotropy values of $\operatorname{Kv} 4.3(2-22)-\operatorname{DREAM}(\Delta 64)$ and $\operatorname{Kv} 4.3(2-22)-$ $\operatorname{DREAM}(\Delta 64)$ in the presence of $\mathrm{Li}^{+}$and $\mathrm{Ca}^{2+}$ suggest that $\mathrm{Li}^{+}$increases affinity between DREAM and $\mathrm{Kv} 4$ channel in a manner analogous to $\mathrm{Ca}^{2+}$. We have also probed if $\mathrm{Li}^{+}$ impacts on DREAM interactions with presenilin-1. A FITC-tagged peptide corresponds to helix-9 of presenilin-1(PS1HL9) were titrated with a saturation concentration of $\operatorname{DREAM}(\Delta 64)(100 \mu \mathrm{M})$ (Figure 5.10C). Anisotropy value of PS1HL9 increases from $0.068 \pm 0.004$ in the absence of protein to $0.095 \pm 0.003$ in the presence of apoDREAM 

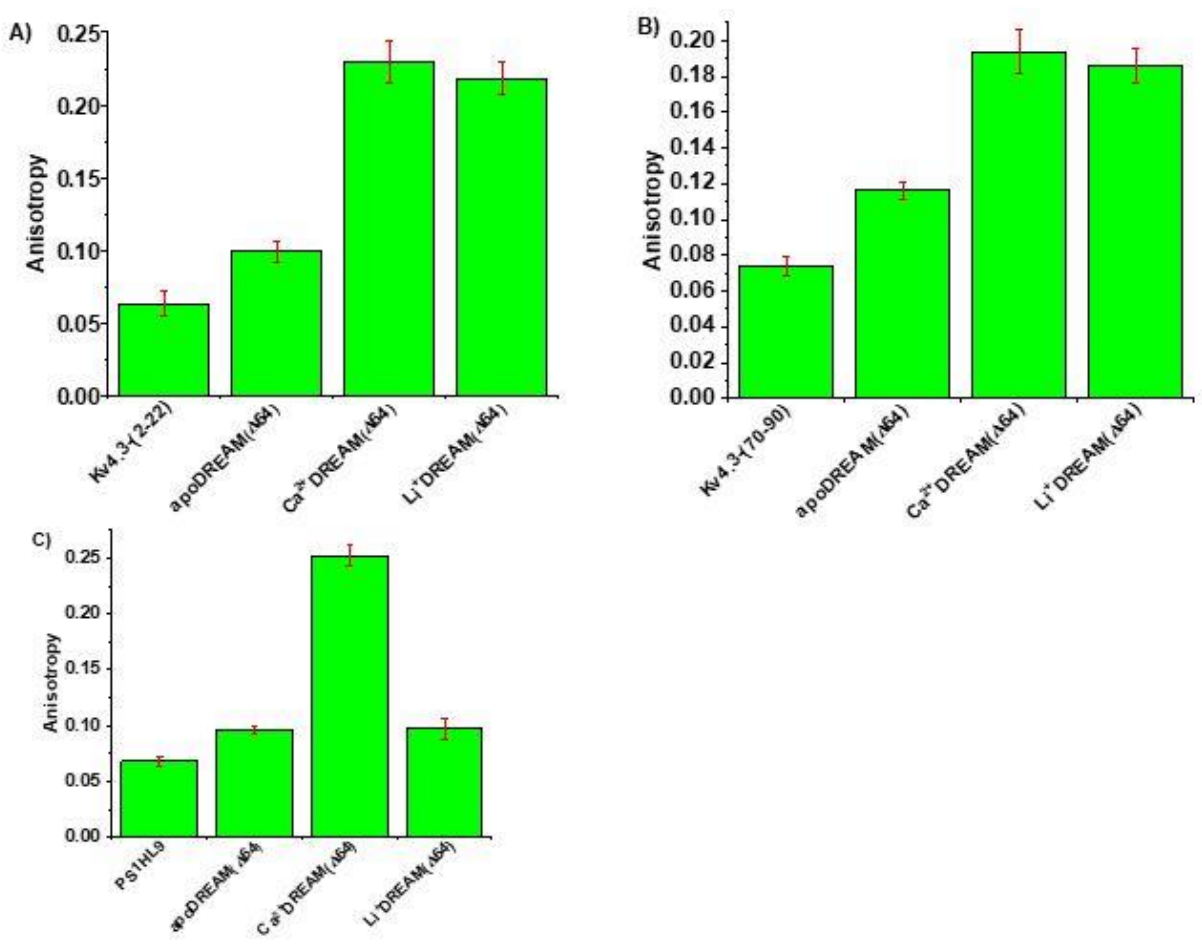

Figure 5.10. Impact of $\mathrm{Li}^{+}$association with DREAM on DREAM interactions with interacting partners. $100 \mu \mathrm{M}, 200 \mu \mathrm{M}$, and $100 \mu \mathrm{M}$ $\operatorname{DREAM}(\Delta 64)$ were respectively added to FITC-labeled A) $0.5 \mu \mathrm{M} \mathrm{K}_{\mathrm{v}} 4.3-$ (2-22). B) $0.5 \mu \mathrm{M} \mathrm{K} \mathrm{v}_{\mathrm{v}} 4.3-(70-90)$, and C) $0.5 \mu \mathrm{M}$ PS1HL9. Samples were excited at $470 \mathrm{~nm}$; emission was recorded through $500 \mathrm{~nm}$ long-pass filter. Concentrations of EDTA, $\mathrm{Ca}^{2+}$, and $\mathrm{Li}^{+}$were same as Trp emission study.

and $0.252 \pm 0.009$ in the presence of $\mathrm{Ca}^{2+}$ DREAM, and $0.097 \pm 0.009$ upon binding to $\operatorname{Li}^{+} \operatorname{DREAM}(\Delta 64)$. Similar anisotropy values of metal-free DREAM $(\Delta 64)-\mathrm{PS} 1 \mathrm{HL} 9$ and $\mathrm{Li}^{+} \operatorname{DREAM}(\Delta 64)-\mathrm{PS} 1 \mathrm{HL} 9$ indicate that $\mathrm{Li}^{+}$association to DREAM doesn't enhance DREAM interaction with presenilin-1. Although additional studies are necessary, these results are consistent with the fact that $\mathrm{Ca}^{2+}$ association to DREAM facilitates DREAM interaction with presenilin-1, resulting in an increase in the accumulation of $\mathrm{A} \beta 42$, a peptide whose overproduction has been linked to Alzheimer's disease, whereas $\mathrm{Li}^{+}$ administration has been shown to decrease $A \beta 42$ production (Jo, Jang et al. 2005, 
Rockenstein, Torrance et al. 2007, Su, Ryder et al. 2004). These results may provide a first molecular insight into the neuroprotective role of $\mathrm{Li}^{+}$.

\subsection{Summary}

Utilizing a combination of fluorescence and calorimetric techniques, we first time comprehensively demonstrate that $\mathrm{Li}^{+}$binds at EF-hand of DREAM; $\mathrm{Li}^{+}$also modulates DREAM interaction with Kv4 channel. Our findings could open up a new avenue for NMR and X-ray crystallographic studies for obtaining detailed structural information of $\mathrm{Li}^{+}$DREAM. Our study also encourages in vivo study in an animal model for investigating the impact of $\mathrm{Li}^{+}$administration on DREAM expression and the impact of $\mathrm{Li}^{+}$treatment on DREAM interactions with interacting partners. Our findings also re-emphasized the potential of $\mathrm{Li}^{+}$for the treatment of manic disorders and neurodegenerative diseases. 


\section{INVESTIGATION OF FEW OTHER METALS BINDING TO DREAM.}

\subsection{Background and significance}

$\mathrm{Mn}^{2+}$ is a transitional metal which is a cofactor of various enzymes. It is essential to support basic physiological function in the cells (Martinez-Finley, Gavin et al. 2013). However, overexposure to $\mathrm{Mn}^{2+}$ leads to its accumulation in the brain, which causes neurotoxicity to several brain regions, including the hippocampus, frontal cortex, substantia nigra, and striatum (Robison, Zakharova et al. 2012, Tuschl, Mills et al. 2013). Studies have suggested that elevated $\mathrm{Mn}^{2+}$ level has been associated with Alzheimer's disease (Tong, Yang et al. 2014). Studies on non-human primate brain demonstrated that chronic $\mathrm{Mn}^{2+}$ exposure leads to aggregation of amyloid- $\beta$ in the frontal cortex, indicating overexposure to $\mathrm{Mn}^{2+}$ might be related to cognitive and memory deficits (Guilarte 2010). But the underlying mechanism of $\mathrm{Mn}^{2+}$-induced neurotoxicity has not been resolved yet. $\mathrm{Mn}^{2+}$ accumulation could influence the homeostasis of the brain microenvironment and has been implicated in cognitive impairment, developmental disorders and neurodegenerative diseases (Yu, Zhou 2018, Lucchini, Placidi et al. 2017). $\mathrm{Mn}^{2+}$ can replace $\mathrm{Mg}^{2+}$ from the active center of different enzymes including DNA-polymerase, RNA-polymerase, adenylate and guanylate cyclases (Bähre, Danker et al. 2014, Lakhin, Efremova et al. 2013, Lakhin, Tarantul et al. 2014). Displacement of $\mathrm{Mg}^{2+}$ from the active center of enzyme is a probable mechanism of $\mathrm{Mn}^{2+}$ intoxication. Besides the alteration of brain microenvironment homeostasis, the promotion of oxidative stress and the generation of reactive oxygen species are considered the possible mechanism of action (Milatovic, Yin et al. 2007, Gunter, Gavin et al. 2006, Archibald, Tyree 1987). It has been shown that $\mathrm{Mn}^{2+}$ binds to EF-hands in $\mathrm{Ca}^{2+}$ binding protein calmodulin and EF-hand protein calprotectin, 
which is a heterodimer of S100A8 and S100A9 (Senguen, Grabarek 2012, Gilston, Skaar et al. 2016). Interestingly, calprotectin has a higher affinity for $\mathrm{Mn}^{2+}$ in the presence of $\mathrm{Ca}^{2+}$ compared with the corresponding metal-free form (Brophy, Nolan 2015, Damo, Kehl-Fie et al. 2013). Since $\mathrm{Mn}^{2+}$ impacts the hippocampus region of the brain and associates to EFhand proteins and DREAM is located in the hippocampus and it's an EF-hand protein, we investigated whether $\mathrm{Mn}^{2+}$ binds to DREAM and alters its tertiary structural rearrangements and impacts interaction with hydrophobic molecule both in the presence and absence of $\mathrm{Ca}^{2+}$.

Chronic arsenic exposure from groundwater is considered one of the worst environmental disorders in the world. More than 100 million people in the world, especially South Asian countries Bangladesh and India, are at risk of cancers and other arsenic-related diseases (Jain, Ali 2000, von Brömssen, Jakariya et al. 2007, Argos, Kalra et al. 2010). The United States Environmental Protection Agency (EPA) and International Agency for Research on Cancer (IARC) have classified arsenic as a human carcinogen. Chronic arsenic exposure also has been associated with noncancerous effects (Chen, Wang et al. 2007, Chen, Graziano et al. 2011). It is widely considered that inorganic arsenate, a molecular analog of phosphate, can replace phosphate in some biochemical reactions (Hughes 2002). For instance, the substitution of phosphate with arsenate could inhibit the generation of adenosine 5'-triphosphate (ATP) during oxidative phosphorylation (Hughes 2002). Trivalent arsenic species have been shown to associate with protein due to high affinity for sulfhydryl groups (Summers 2009, Kitchin, Wallace 2005, Zhao, Chen et al. 2012). Arsenic binding to a particular protein could alter its conformation and interaction with interacting partners which could be a possible mechanism of arsenic toxicity. Arsenic binding to 
protein has been associated with inactivation of about 200 enzymes, including glutathione S-transferase, glutathione peroxidase, glutathione reductase, thioredoxin reductase, thioredoxin peroxidase, pyruvate kinase galectin-1 (Ratnaike 2003, Webb 1966, Müller, Walter et al. 1995, Chouchane, Snow 2001, Lu, Chew et al. 2007, Chang, Lee et al. 2003, Lin, Huang et al. 2006). Trivalent arsenic species also have been demonstrated to inhibit the activities of various DNA repair proteins such as formamidopyrimidine-DNA glycosylase (Fpg) and xeroderma pigmentosum group A protein (XPA). Each of these proteins comprises a zinc finger DNA binding domain (Walter, Schwerdtle et al. 2007, Hartwig, Blessing et al. 2003).The association of arsenic compounds with these repair proteins resulted in the release of zinc. On the other hand, arsenic compounds have been used as drugs for more than 2400 years (Doyle 2009, Waxman, Anderson 2001). Particularly, $\mathrm{As}_{2} \mathrm{O}_{3}$ has been used for the treatment of patients suffering from acute promyelocytic leukemia (APL), probably because of arsenic association with cysteine residues in zinc fingers of the aberrant promyelocytic leukemia-retinoic acid receptor (PML-RAR) fusion protein expressed by these patients (Zhang, Yan et al. 2010, Jeanne, Lallemand-Breitenbach et al. 2010). Since our study demonstrated toxic metals such as lead and cadmium bind to DREAM (Azam, Miksovska 2018, Azam, St Luis et al. 2019), in this case, we investigated whether $\mathrm{As}^{5+}$ binds to DREAM and triggers tertiary structural rearrangements.

Mercury is a very toxic metal, which naturally presents in the environment (Clarkson 2002). It exists in three different forms, namely, elemental mercury (0), inorganic mercury (I, II), and organic mercury. Among them, organic mercury is considered supertoxic (Park, Zheng 2012, Carocci, Rovito et al. 2014). After exposure, mercury is translocated to the bloodstream and important organs, ultimately causing kidney, 
cardiovascular, gastrointestinal, immune, and nervous system malfunction (Clarkson 2002). The toxic effect exerted by numerous mercury compounds could be best explained by binding of $\mathrm{Hg}^{2+}$ to cysteine-thiol and seleno-cysteine residues of critical enzymes (Jan, Ali et al. 2011, Branco, Canário et al. 2012, Carvalho, Chew et al. 2008, Clarkson, Magos 2006). Mercury binding causes dramatic changes to the SH group as mercury is much larger than the $\mathrm{H}$ that it displaces. If the $\mathrm{SH}$ is essential for enzyme activity, binding of $\mathrm{Hg}^{2+}$ causes inhibition of that activity. For instance, binding of mercury causes inhibition of protein kinase C activity (Saijoh, Inoue et al. 1988). Binding of mercury to the SH group of protein can also lead to unfolding, precipitation, and aggregation of the protein (Konyaeva, Myshkin 1998a, Khromova, Myshkin 2000). In addition to binding to cysteine residues, $\mathrm{Hg}^{2+}$ has been shown to bind to non-cysteine residues of some proteins, including hemoglobin and chymotrypsin. Binding of $\mathrm{Hg}^{2+}$ to non-cysteine residues protects hemoglobin against aggregation (Konyaeva, Myshkin 1998c, Konyaeva, Myshkin 1998b, Stratton, Ericksen et al. 2017, Myshkin, Khromova 2000). In the absence of inaccessible cysteine residues, $\mathrm{Hg}^{2+}$ binds to histidine residues of digestive enzyme chymotrypsin, which causes denaturation of the enzyme (Stratton, Ericksen et al. 2017). Considering the similar ionic radii of $\mathrm{Pb}^{2+}, \mathrm{Cd}^{2+}$, and $\mathrm{Hg}^{2+}$ and the previously reported binding of $\mathrm{Hg}^{2+}$ to calcium binding sites on CaM (Vig, Nath et al. 1989), we set forth to determine whether heavy metal $\mathrm{Hg}^{2+}$ binds to DREAM and induces tertiary structural rearrangements both in the presence and absence of $\mathrm{Ca}^{2+}$.

Alkaline earth metals $\mathrm{Sr}^{2+}$ and $\mathrm{Ba}^{2+}$ have been shown to bind to EF-hand protein $\mathrm{CaM}$ and calcium-binding protein-1 from Entamoeba histolytica (EhCaBP1) (Kursula, Majava 2007, Kumar, Ahmad et al. 2012). Among different EF-hands of DREAM, $\mathrm{Mg}^{2+}$ 
binds to EF-2 selectively, whereas EF-3 and EF-4 bind $\mathrm{Ca}^{2+}$ preferentially. Since $\mathrm{Sr}^{2+}$ and $\mathrm{Ba}^{2+}$ have similar ionic radii $(1.21$ and $1.38 \AA$, respectively $)$ as $\mathrm{Ca}^{2+}(1.06 \AA)(\mathrm{Gu}$, Cooper 2000), we set out to determine whether $\mathrm{Sr}^{2+}$ and $\mathrm{Ba}^{2+}$ bind to EF-hand of DREAM and trigger tertiary structural rearrangement of the protein.

\subsection{Results and discussion}

Tryptophan emission is a widely-used tool to probe structural rearrangement of the proteins. Our previous studies have shown that DREAM undergoes structural rearrangement upon binding of $\mathrm{Ca}^{2+}, \mathrm{Pb}^{2+}, \mathrm{Cd}^{2+}$, and $\mathrm{Tb}^{3+}$ in the EF-hand of the protein and emission intensity of tryptophan decreases upon binding of above metals (Azam, Miksovska 2018, Azam, St Luis et al. 2019, Gonzalez, Ramos et al. 2016, Pham, Dhulipala et al. 2015). We have also demonstrated that hydrophobic cavities of DREAM become more accessible to hydrophobic molecules 1,8-ANS in the presence of above metals compared with metal-free form. Consequently, the emission intensity of DREAM:1,8-ANS is higher in the presence of above metals compared with corresponding metal-free form (Azam, Miksovska 2018, Azam, St Luis et al. 2019, Gonzalez, Ramos et al. 2016, Gonzalez, Miksovska 2014). To investigate whether $\mathrm{As}^{5+}, \mathrm{Hg}^{2+}, \mathrm{Mn}^{2+}, \mathrm{Ba}^{2+}$, or $\mathrm{Sr}^{2+}$ binds to DREAM, we added $1 \mathrm{mM}$ of barium chloride, strontium chloride, arsenic pentoxide, mercuric oxide and manganese chloride into $\operatorname{DREAM}(\Delta 64)$ protein and $\operatorname{DREAM}(\Delta 64)$ :1,8-ANS complex both in the presence and absence of $\mathrm{Ca}^{2+}$ (Figure 6.16.5A-D). Our results show that addition of $\mathrm{As}^{5+}, \mathrm{Hg}^{2+}, \mathrm{Mn}^{2+}, \mathrm{Ba}^{2+}$ or $\mathrm{Sr}^{2+}$ doesn't alter emission intensity of $\operatorname{DREAM}(\Delta 64)$ or emission intensity of $\operatorname{DREAM}(\Delta 64): 1,8$-ANS, suggesting either $\mathrm{As}^{5+}, \mathrm{Hg}^{2+}, \mathrm{Mn}^{2+}, \mathrm{Ba}^{2+}$, or $\mathrm{Sr}^{2+}$ does not bind to $\operatorname{DREAM}(\Delta 64)$ or binding of $\mathrm{As}^{5+}, \mathrm{Hg}^{2+}, \mathrm{Mn}^{2+}, \mathrm{Ba}^{2+}$, or $\mathrm{Sr}^{2+}$ does not trigger structural rearrangements of DREAM. 

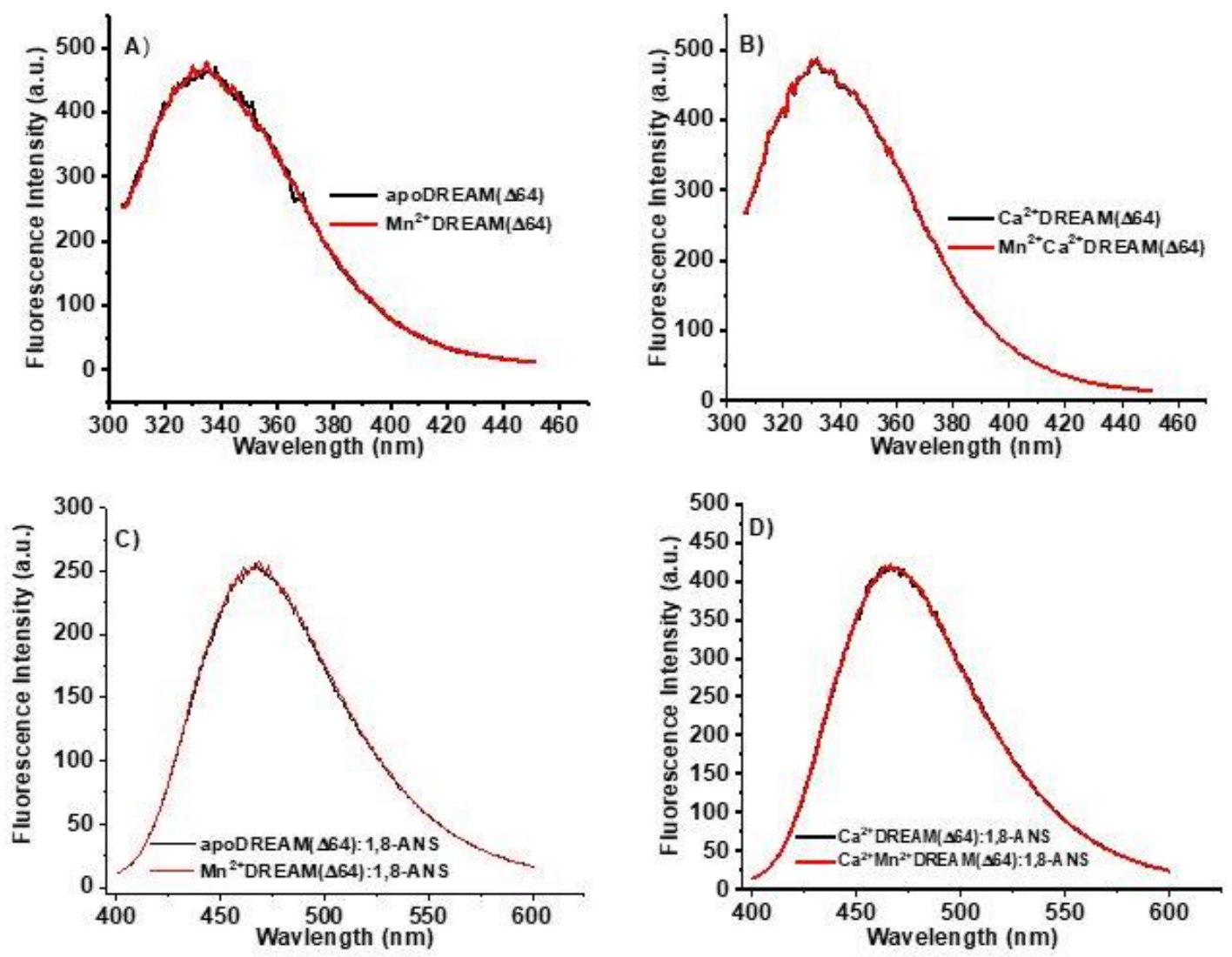

Figure 6.1. Impact of $1 \mathrm{mM} \mathrm{Mn}^{2+}$ addition on Trp emission of metal-free and $\mathrm{Ca}^{2+}$-bound $\operatorname{DREAM}(\Delta 64) \quad(\mathrm{A}, \mathrm{B})$ and emission of metal free and $\mathrm{Ca}^{2+}$-bound $\operatorname{DREAM}(\Delta 64): 1,8$ ANS (C,D). 

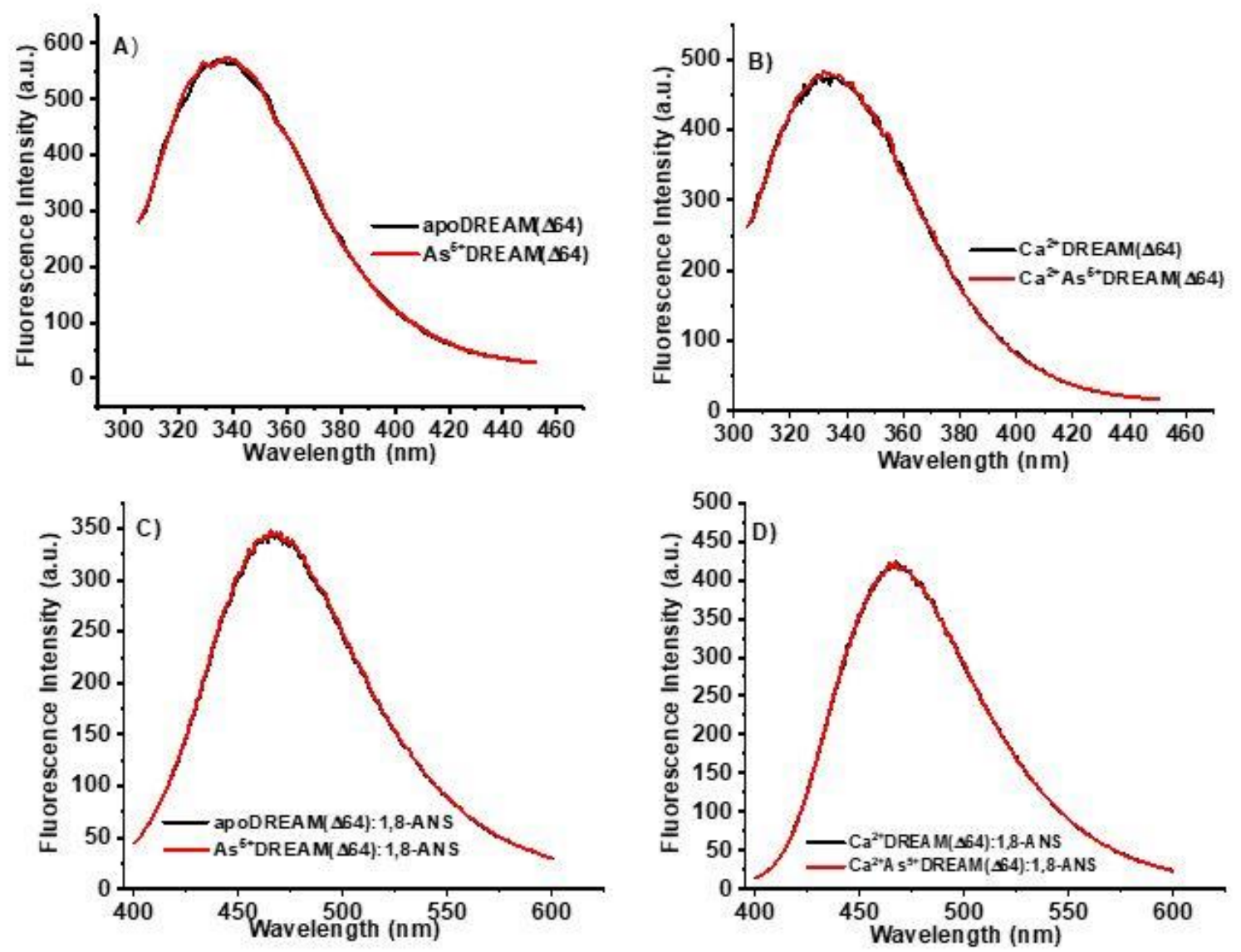

Figure 6.2. Impact of $1 \mathrm{mM} \mathrm{As}^{5+}$ addition on Trp emission of metal-free and $\mathrm{Ca}^{2+}$-bound $\operatorname{DREAM}(\Delta 64)(\mathrm{A}, \mathrm{B})$ and emission of metal free and $\mathrm{Ca}^{2+}$-bound $\operatorname{DREAM}(\Delta 64)$ :1,8-ANS (C,D). 

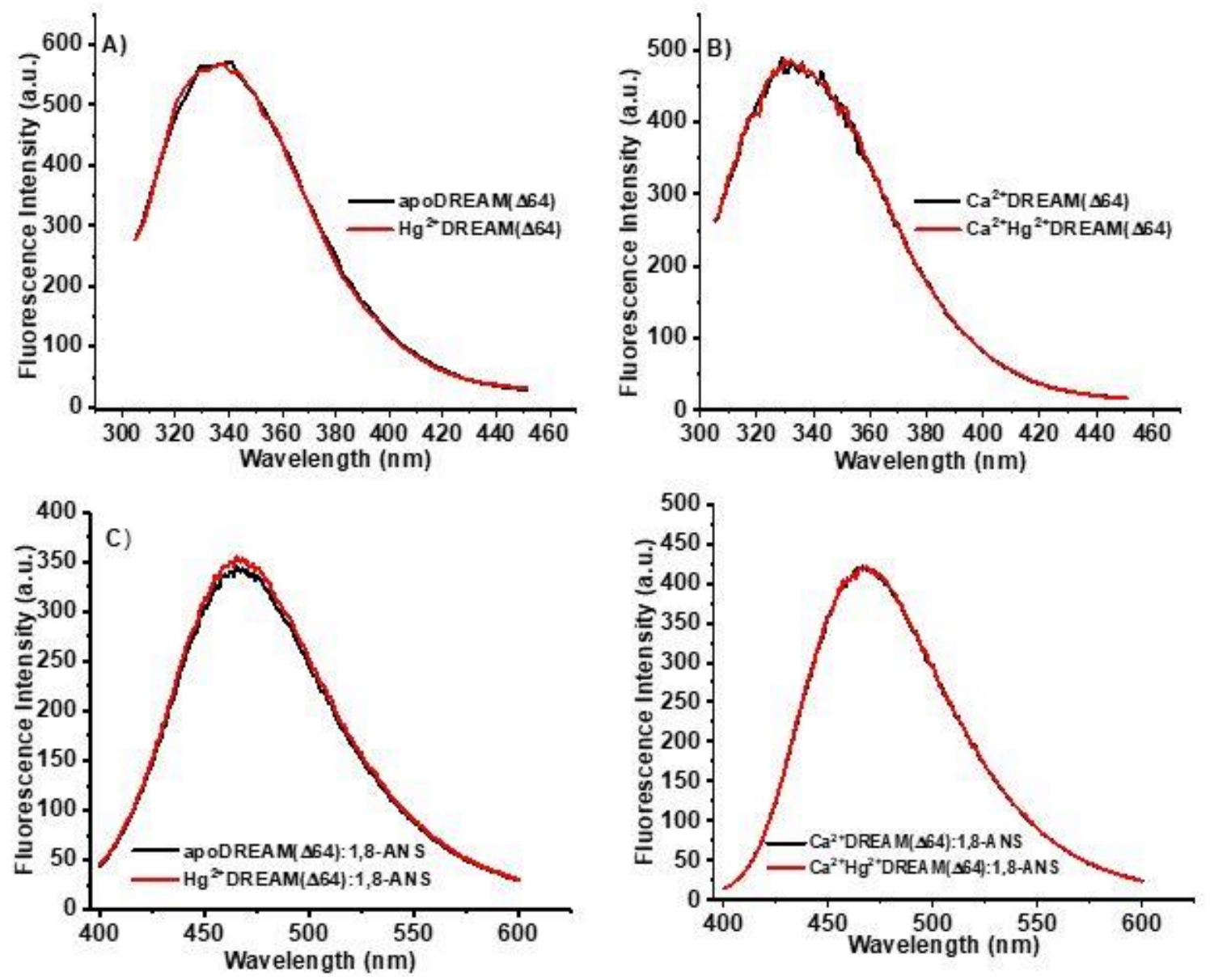

Figure 6.3. Impact of $1 \mathrm{mM} \mathrm{Hg}^{2+}$ addition on Trp emission of metal-free and $\mathrm{Ca}^{2+}$-bound $\operatorname{DREAM}(\Delta 64)(\mathrm{A}, \mathrm{B})$ and emission of metal free and $\mathrm{Ca}^{2+}$-bound $\operatorname{DREAM}(\Delta 64): 1,8$-ANS (C,D). 

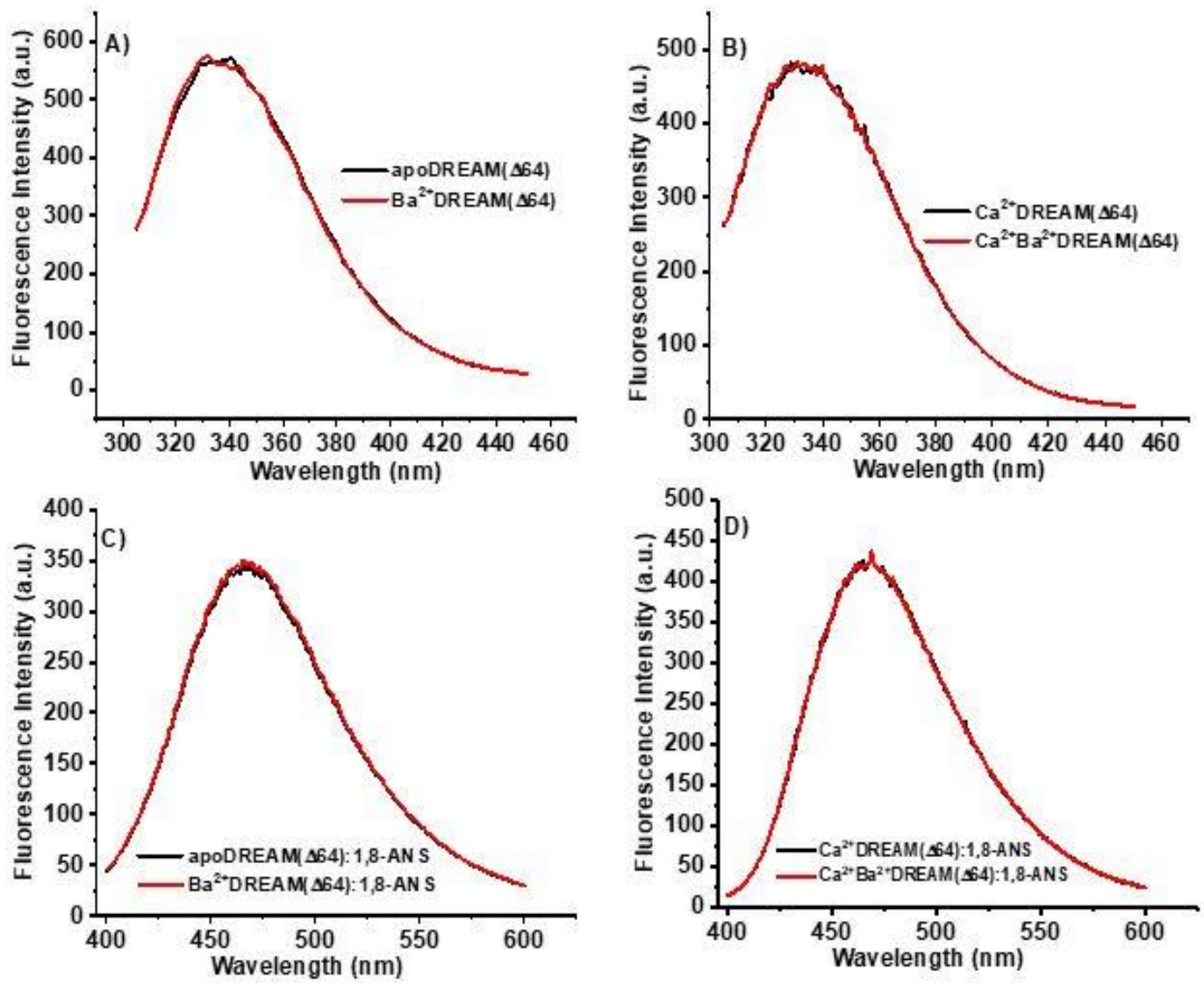

Figure 6.4. Impact of $1 \mathrm{mM} \mathrm{Ba}^{2+}$ addition on Trp emission of metal-free and $\mathrm{Ca}^{2+}$-bound $\operatorname{DREAM}(\Delta 64)(\mathrm{A}, \mathrm{B})$ and emission of metal free and $\mathrm{Ca}^{2+}$-bound $\operatorname{DREAM}(\Delta 64): 1,8$-ANS $(\mathrm{C}, \mathrm{D})$. 

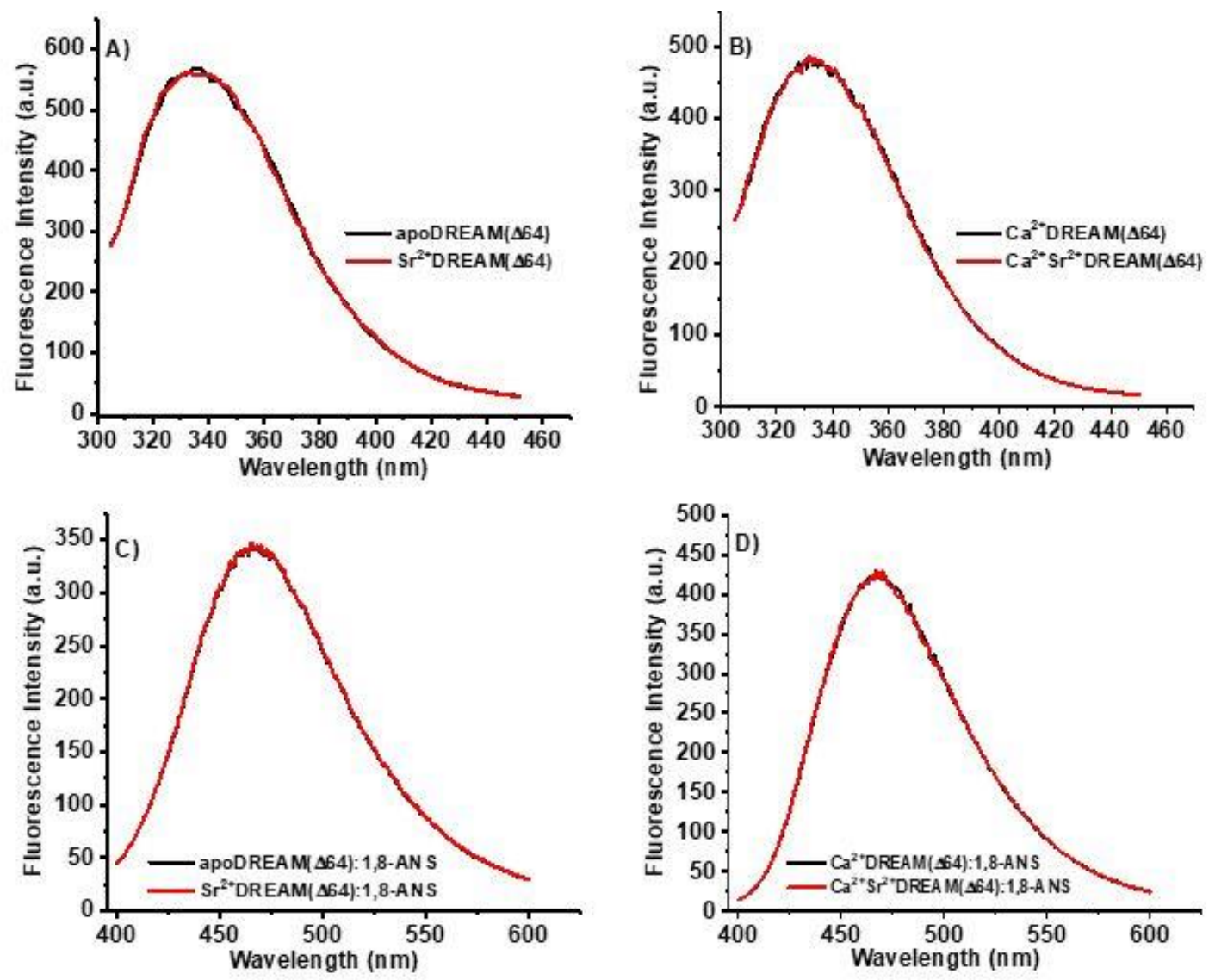

Figure 6.5. Impact of $1 \mathrm{mM} \mathrm{Sr}^{2+}$ addition on Trp emission of metal-free and $\mathrm{Ca}^{2+}$-bound $\operatorname{DREAM}(\Delta 64)(\mathrm{A}, \mathrm{B})$ and emission of metal free and $\mathrm{Ca}^{2+}$-bound $\operatorname{DREAM}(\Delta 64): 1,8$ ANS (C,D). 


\subsection{Summary}

Encouraged by the fact that toxic metals $\mathrm{Cd}^{2+}$ and $\mathrm{Pb}^{2+}$ bind to DREAM, we investigated whether toxic metals $\mathrm{Mn}^{2+}, \mathrm{As}^{5+}$, and $\mathrm{Hg}^{2+}$ bind to DREAM. Additionally, we investigated whether alkaline earth metals $\mathrm{Ba}^{2+}$ and $\mathrm{Sr}^{2+}$ bind to DREAM. On the basis of the fact that addition of $\mathrm{As}^{5+}, \mathrm{Hg}^{2+}, \mathrm{Mn}^{2+}, \mathrm{Ba}^{2+}$, or $\mathrm{Sr}^{2+}$ did not change the emission intensity of DREAM $(\Delta 64)$ or DREAM( $\Delta 64): 1,8$-ANS complexes, we conclude that either $\mathrm{As}^{5+}$, $\mathrm{Hg}^{2+}, \mathrm{Mn}^{2+}, \mathrm{Ba}^{2+}$, or $\mathrm{Sr}^{2+}$ does not bind to DREAM or addition of $\mathrm{As}^{5+}, \mathrm{Hg}^{2+}, \mathrm{Mn}^{2+}, \mathrm{Ba}^{2+}$, or $\mathrm{Sr}^{2+}$ does not trigger structural rearrangements of DREAM. 
7. ROLE OF LYS RESIDUE AT POSITION 87 OF DREAM IN ALLOSTERIC REGULATION OF DREAM INTERACTIONS WITH INTERACTING PARTNERS.

\subsection{Background and significance}

Downstream regulatory element antagonist modular (DREAM), alternative known as KChIP3/calsenilin, is a 29kDa NCS protein which is expressed in the brain and heart and regulate a wide spectrum of biological processes (An, Bowlby et al. 2000, Buxbaum, Choi et al. 1998, Carrion, Link et al. 1999). DREAM is the only $\mathrm{Ca}^{2+}$ binding protein that can directly interact with DNA sequence (DRE) of genes (prodynorphin and c-fos genes) and represses transcription of these genes (Carrion, Link et al. 1999). In the absence of $\mathrm{Ca}^{2+}$, DREAM interacts with DRE of human prodynorphin and c-fos genes and inhibit transcription of these genes, but $\mathrm{Ca}^{2+}$ association induces conformation change in the protein that prevents binding of DRE and consequently reactivates the transcription (Carrion, Link et al. 1999, Cheng, Pitcher et al. 2002). Prodynorphin and c-fos genes have been linked to pain modulation, cell homeostasis, and apoptosis. Repression of the transcription of DRE by DREAM highlights its role in the regulation of the above processes (Costigan, Woolf 2002, Fontán-Lozano, Romero-Granados et al. 2009).

In the cytoplasm, DREAM interacts with carboxy-terminal fragment (CTF) of presenilin-1 (PS1) and presenilin-2 and stimulates the activity of $\gamma$ - secretase complex in a $\mathrm{Ca}^{2+}$-dependent manner, which facilitates production of amyloid-beta plaques of Alzheimer's disease (Buxbaum, Choi et al. 1998, Jo, Jang et al. 2005). DREAM also associates with the $\mathrm{T} 1$ domain of voltage-gated potassium channels and plays a 
crucial role in the regulation of fast $\mathrm{I}_{\mathrm{TO}}$ and $\mathrm{I}_{\mathrm{SA}}$ current in the heart and brain, respectively. The above interaction also promotes the translocation of the channel to the membrane and modulates the gating properties of the channel (An, Bowlby et al. 2000). It has been shown recently that DREAM protein might be involved in the mechanism of nicotine treatment-prevented learning and memory impairment in REM sleep-deprived rats by modulating its expression level in the hippocampus (Abd Rashid, Hapidin et al. 2017).

DREAM protein contains 256 amino acid residues. 3-D structure of the fulllength DREAM is not known yet because its poor solubility prevented structural determination of the first 77 residues by NMR-studies (Lusin, Vanarotti et al. 2008). After the deletion of the first 64 residues, the solution structure of $\mathrm{Ca}^{2+}$ DREAM (residue 65-256) was resolved (Lusin, Vanarotti et al. 2008). That's why in our study we used DREAM protein that possesses residues 65-256. DREAM protein contains four EF-hands. Each EFhand has a distinct metal binding property. EF-1 doesn't bind any metal because it has sterically hindered proline residues in the EF-hand loop. EF-2 binds $\mathrm{Mg}^{2+}$ preferentially as it has aspartate residue in the $12^{\text {th }}$ position of the EF-hand loop. EF-3 and EF-4 bind $\mathrm{Ca}^{2+}$ selectively because glutamate residue is present in the $12^{\text {th }}$ position of the EF-hand loop (Osawa, Dace et al. 2005, Osawa, Tong et al. 2001). Recently, we have shown that EFhands of DREAM are also capable of binding heavy metals $\mathrm{Pb}^{2+}$ and $\mathrm{Cd}^{2+}(\mathrm{Azam}$, Miksovska 2018, Azam, St Luis et al. 2019)

Our previous studies demonstrated that DREAM undergoes tertiary structural rearrangement upon $\mathrm{Ca}^{2+}, \mathrm{Tb}^{3+}, \mathrm{Pb}^{2+}$, or $\mathrm{Cd}^{2+}$ binding. We also have shown hydrophobic cavities of DREAM that binds hydrophobic molecule 1,8-ANS becomes more accessible 
to 1,8 -ANS in the presence of $\mathrm{Ca}^{2+}, \mathrm{Tb}^{3+}, \mathrm{Pb}^{2+}$, or $\mathrm{Cd}^{2+}$ association, compared with metalfree form (Azam, Miksovska 2018, Azam, St Luis et al. 2019, Gonzalez, Ramos et al. 2016, Gonzalez, Miksovska 2014). Additionally, we demonstrated that helix-9 of presenilin-1 is involved in DREAM-presenilin-1 interaction and site-1 (residues 2-22) and site-2 (residues 70-90) peptides of T-1 domain of Kv4.3 channel are involved in DREAM- Kv4.3 channel interaction (Pioletti, Findeisen et al. 2006, Gonzalez, Pham et al. 2014, Pham, Miksovska 2016, Wang, Yan et al. 2007a). We have used accelerated molecular dynamics to get insight about the mechanism of allosteric regulation of DREAM
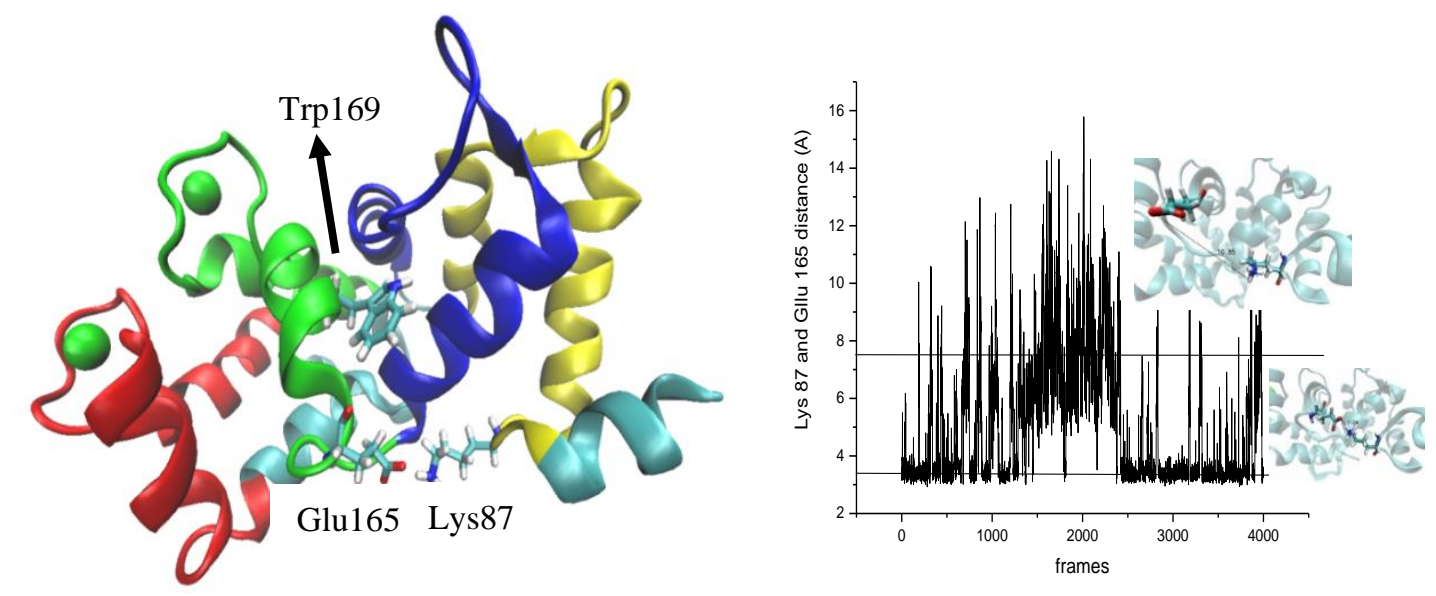

Figure 7.1. Left: aMD structure of $\mathrm{Ca}^{2+}$ DREAM. EF-hands are shown in different colors: EF-hand 1, yellow; EF-hand 2, blue; EF-hand 3, green; EF-hand 4, red; rest part of the protein is shown in cyan; two $\mathrm{Ca}^{2+}$ are shown as green spheres; Trp169, Glu165, and Lys87 are show as sticks. Right: Distance between Lys87 and Glu165 side chains determined from $100 \mathrm{~ns}$ accelerated molecular simulation of $\operatorname{DREAM}(\Delta 64)$.

interactions with intracellular partners. Unlike the NMR structure of DREAM, aMD data indicate the presence of a salt bridge between Lys87 and Asp 165 that couples the loop between helix-1 and helix-2 in the N-terminal domain with the EF-3 in the C-terminal domain (Figure 7.1). We hypothesize that the salt bridge involved in the propagation of 
calcium-triggered structural changes between the $\mathrm{C}$ - terminal and $\mathrm{N}$ - terminal domain. To determine the contribution of Lys87 to the interdomain communication Lys87 was mutated to Ala and the impact of the mutation on DREAM secondary and tertiary structures and DREAM interactions with interacting partners were investigated.

\subsection{Results and discussion}

7.2.1 Impact of Lys87 to Ala mutation on DREAM secondary and tertiary structures.

It has been well established from our previous study that emission maximum of wild-type DREAM $(\Delta 64)$ in metal-free form is $335 \mathrm{~nm}$ and emission maximum shifts to $329 \mathrm{~nm}$ upon $\mathrm{Ca}^{2+}$ binding (Azam, Miksovska 2018, Gonzalez, Ramos et al. 2016, Osawa, Tong et al. 2001). Emission maximum of Trp169 in DREAM(K87A) is $5 \mathrm{~nm}$ red-shifted compared with corresponding wild-type protein, suggesting Trp169 is more solvent exposed in DREAM(K87A) (Figure 7.2A). The increase in solvent accessibility can be explained by the fact that the absence of salt bridge destabilizes the protein structure. $\mathrm{Ca}^{2+}$ binding to DREAM(K87A) leads to decrease in ellipticity which is similar to $\mathrm{Ca}^{2+}$ binding
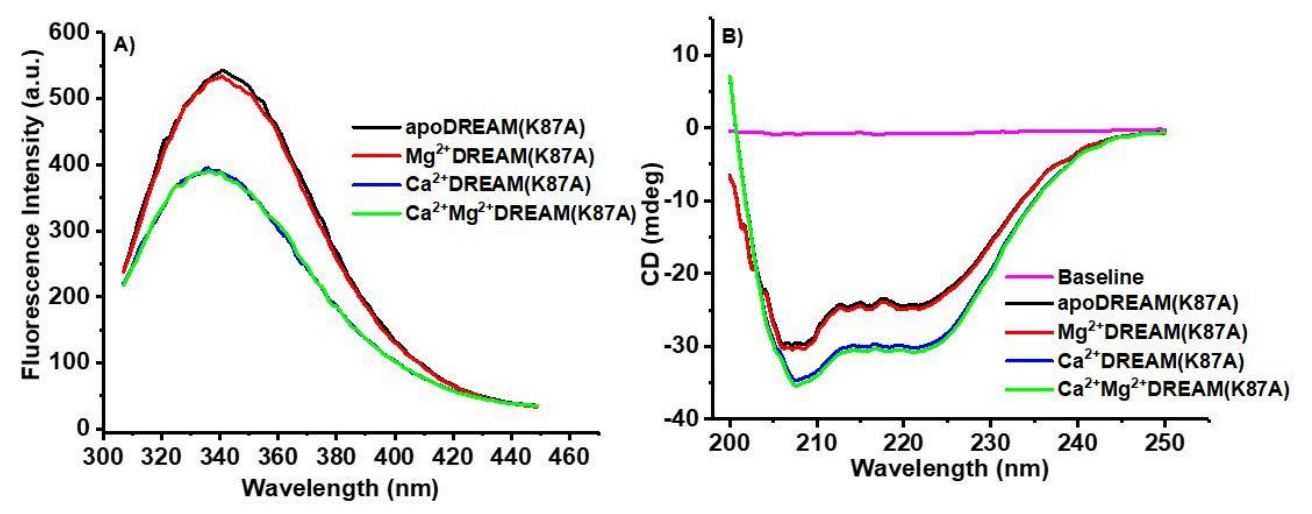

Figure 7.2. (A) Intrinsic fluorescence of $20 \mu \mathrm{M}$ DREAM(K87A) in the presence of $1 \mathrm{mM}$ EDTA, $5 \mathrm{mM} \mathrm{Mg}^{2+}$, and $1 \mathrm{mM} \mathrm{Ca}^{2+}$; excited at $295 \mathrm{~nm}$. (B) Far-UV CD spectra of DREAM(K87A) under identical condition.

to corresponding WTDREAM( $\Delta 64)$ (Figure 7.2B). Analyzing of the far-UV CD data with secondary structure prediction tool K2D3 reveals that DREAM(K87A) contains 53\%, 
$54 \%, 63 \%$, and $65 \% \alpha$-helical character in apo, $\mathrm{Mg}^{2+}, \mathrm{Ca}^{2+}$, and $\mathrm{Ca}^{2+} \mathrm{Mg}^{2+}$-bound form, respectively. Interestingly, both metal-free and metal-bound form of DREAM(K87A) show about $10 \%$ less $\alpha$-helical character than corresponding WTDREAM $(\Delta 64)$, suggesting that the disruption of the salt bridge makes the protein more flexible. Analogously, emission spectra of 1,8-ANS in the presence of DREAM(K87A) is $10 \mathrm{~nm}$ red-shifted compared with corresponding wild-type protein, indicating the hydrophobic cavity that binds 1,8-ANS is

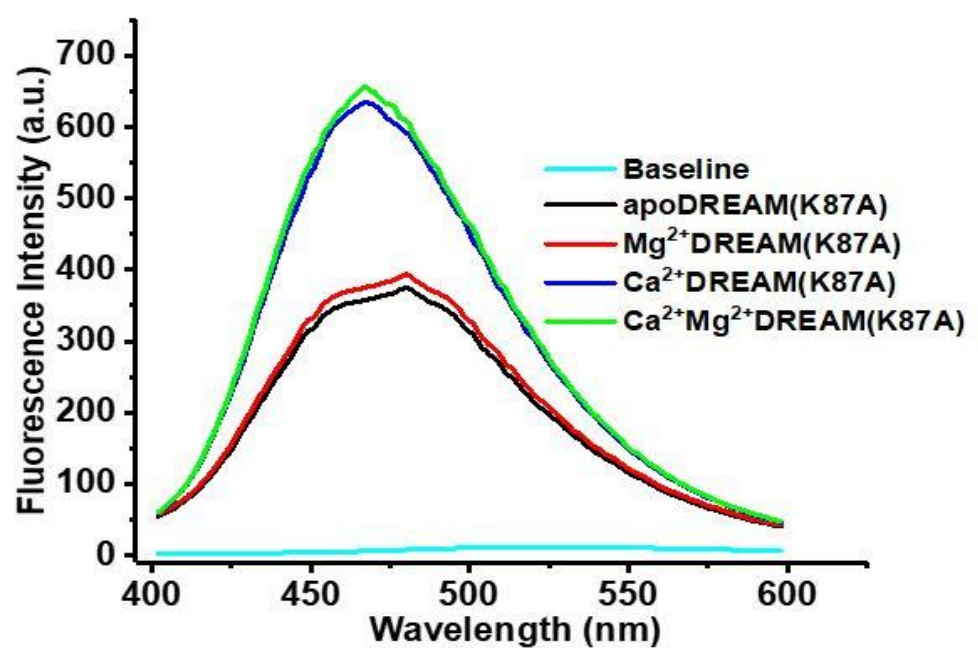

Figure 7.3. Fluorescence emission of $\operatorname{DREAM}(\mathrm{K} 87 \mathrm{~A}): 1,8$ ANS complex. Condition: $20 \mu \mathrm{M}$ DREAM(K87A), $20 \mu \mathrm{M}$ 1,8ANS, excited at $350 \mathrm{~nm}$.

more solvent exposed in DREAM(K87A) (Figure 7.3). However, the decrease in emission intensity of Trp and increase in emission intensity of 1,8-ANS upon calcium addition to metal-free DREAM(K87A) are analogous to calcium addition to metal-free wild-type protein, suggesting that the mutation of Lys to Ala does not prevent Trp transition and 1,8ANS binding to DREAM. Alteration of tertiary structure of the protein is further confirmed by the lifetime data. Analogous to WTDREAM( $\Delta 64)$, W169 residue of DREAM(K87A) exhibits three different lifetimes (Figure 7.4, Table 7.1), which could be due to the presence of multiple rotamers of the indole side chain as well as multiple conformational states 


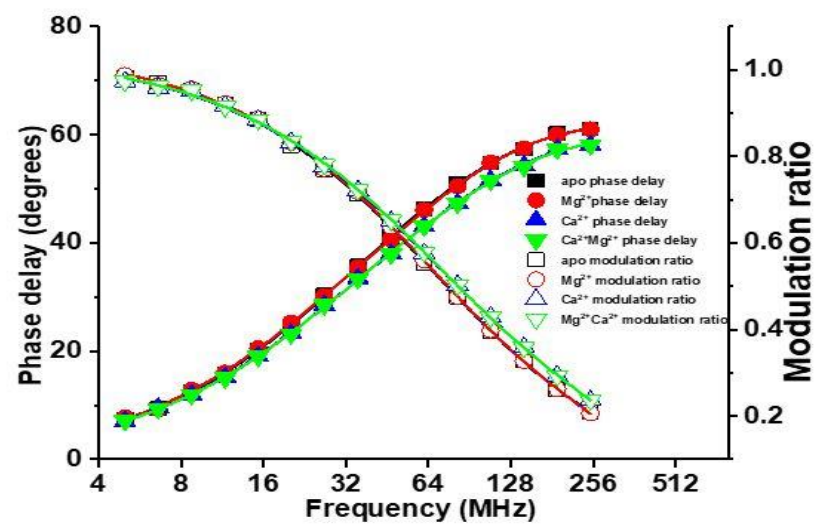

Figure 7.4. Frequency-domain intensity decay of 20 $\mu \mathrm{M}$ DREAM(K87A) in apo (square), $\mathrm{Mg}^{2+}$ (circle), $\mathrm{Ca}^{2+}$ (upper triangle), and $\mathrm{Ca}^{2+} \mathrm{Mg}^{2+}$ (lower triangle) bound form. Solid symbols correspond to phase delay; empty symbols correspond to modulation ratio. Solid lines represent data fitting using a sum of three exponential decays model.

(Pham, Dhulipala et al. 2015). Similar to WTDREAM( $\Delta 64)$, upon $\mathrm{Ca}^{2+}$ binding the average lifetime of W169 in DREAM(K87A) decreases by 0.3 ns, compared with corresponding apo-form, which supports emission data. Analogous to WTDREAM $(\Delta 64)$, 1,8-ANS binds to two different binding sites on DREAM(K87A) surface as evident from two distinct lifetimes of 1,8-ANS in 1,8-ANS:DREAM(K87A) complexes (Figure 7.5, Table 7.2). While the mutation did not change the individual lifetimes of 1,8-ANS significantly, it decreased the affinity for 1,8-ANS, as evident by smaller $\alpha_{1}$ and $\alpha_{2}$ values compared with wild-type protein, likely due to the lower accessibility of the 1,8-ANS binding sites. 


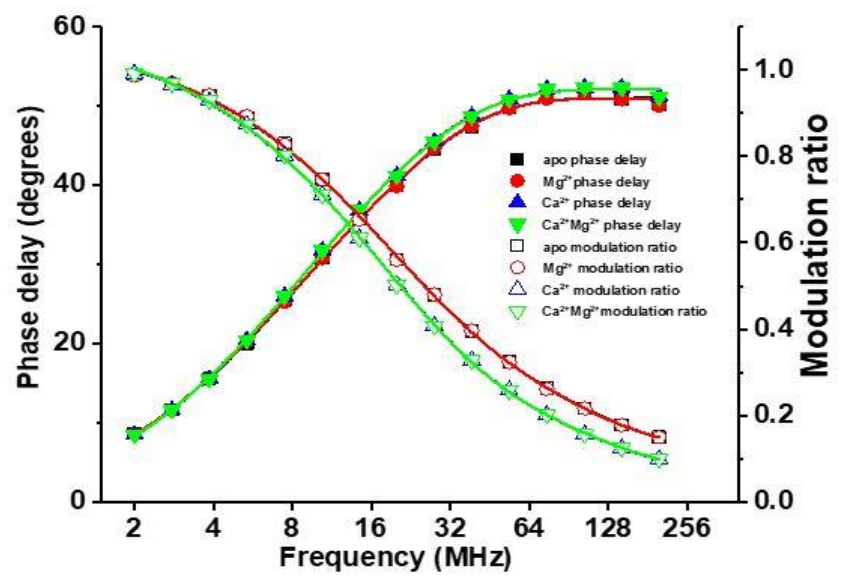

Figure 7.5. Frequency-domain intensity decay of DREAM(K87A):1,8-ANS in apo (square), $\mathrm{Mg}^{2+}$ (circle), $\mathrm{Ca}^{2+}$ (upper triangle), and $\mathrm{Ca}^{2+} \mathrm{Mg}^{2+}$ (lower triangle) bound form. Solid symbols correspond to phase delay; empty symbols correspond to modulation ratio. Solid lines represent data fitting using a sum of three exponential decays model. 
Table 7.1: Fluorescence decay parameters of DREAM(K87A) in the presence and absence of metals.

\begin{tabular}{|c|c|c|c|c|c|c|c|c|c|c|c|}
\hline $\begin{array}{l}\text { Sample } \\
\text { s }\end{array}$ & $\begin{array}{l}\tau_{1} \\
(\mathrm{~ns})\end{array}$ & $\begin{array}{l}\tau_{2} \\
(\mathrm{~ns})\end{array}$ & $\begin{array}{l}\tau_{3} \\
(\mathrm{~ns})\end{array}$ & $\begin{array}{l}\mathrm{f}_{1} \\
(\%)\end{array}$ & $\begin{array}{l}f_{2} \\
(\%)\end{array}$ & $\begin{array}{l}\mathrm{f}_{3} \\
(\%)\end{array}$ & $\begin{array}{l}\alpha_{1} \\
(\%)\end{array}$ & $\begin{array}{l}\alpha_{2} \\
(\%)\end{array}$ & $\begin{array}{l}\alpha_{3} \\
(\%)\end{array}$ & $\begin{array}{l}<\tau> \\
(\mathrm{ns})\end{array}$ & $\chi^{2}$ \\
\hline Apo & $\begin{array}{l}0.32 \pm \\
0.03\end{array}$ & $\begin{array}{l}2.7 \pm \\
0.1\end{array}$ & $\begin{array}{l}7.2 \pm \\
0.3\end{array}$ & $\begin{array}{l}8.9 \pm \\
0.1\end{array}$ & $\begin{array}{l}52.4 \pm \\
0.3\end{array}$ & $\begin{array}{l}38.1 \pm \\
0.2\end{array}$ & 53.2 & 36.9 & 10.1 & 4.2 & 1.9 \\
\hline $\mathrm{Mg}^{2+}$ & $\begin{array}{l}0.30 \pm \\
0.02\end{array}$ & $\begin{array}{l}2.6 \pm \\
0.1\end{array}$ & $\begin{array}{l}7.2 \pm \\
0.4\end{array}$ & $\begin{array}{l}8.4 \pm \\
0.1\end{array}$ & $\begin{array}{l}51.3 \pm \\
0.1\end{array}$ & $\begin{array}{l}40.1 \pm \\
0.3\end{array}$ & 53.4 & 36.4 & 10.2 & 4.2 & 2.1 \\
\hline $\mathrm{Ca}^{2+}$ & $\begin{array}{l}0.28 \pm \\
0.03\end{array}$ & $\begin{array}{l}2.4 \pm \\
0.1\end{array}$ & $\begin{array}{l}7.1 \pm \\
0.2\end{array}$ & $\begin{array}{l}10.5 \pm \\
0.1\end{array}$ & $\begin{array}{l}49.6 \pm \\
0.2\end{array}$ & $\begin{array}{l}38.8 \pm \\
0.2\end{array}$ & 56.3 & 34.5 & 9.1 & 3.9 & 1.7 \\
\hline $\begin{array}{l}\mathrm{Ca}^{2+} \mathrm{M} \\
\mathrm{g}^{2+}\end{array}$ & $\begin{array}{l}0.29 \pm \\
0.03\end{array}$ & $\begin{array}{l}2.4 \pm \\
0.1\end{array}$ & $\begin{array}{l}7.1 \pm \\
0.1\end{array}$ & $\begin{array}{l}10.7 \pm \\
0.1\end{array}$ & $\begin{array}{l}49.8 \pm \\
0.3\end{array}$ & $\begin{array}{l}39.1 \pm \\
0.2\end{array}$ & 56.1 & 34.8 & 8.9 & 3.9 & 1.8 \\
\hline
\end{tabular}




\begin{tabular}{llllllllll}
\hline \multicolumn{7}{l}{ Table 7.2: Fluorescence decay parameters of DREAM(K87A):1,8-ANS complexes. } \\
\hline DREAM(K87A):1,8-ANS & $\tau_{1}$ & $\tau_{2}$ & $\mathrm{f}_{0}$ & $\mathrm{f}_{1}$ & $\mathrm{f}_{2}$ & $\alpha_{0}$ & $\alpha_{1}$ & $\alpha_{2}$ & $\chi^{2}$ \\
& $(\mathrm{~ns})$ & $(\mathrm{ns})$ & $(\%)$ & $(\%)$ & $(\%)$ & $(\%)$ & $(\%)$ & $(\%)$ & \\
\hline apo & $4.0 \pm$ & $16.4 \pm$ & $8.9 \pm$ & $33.4 \pm$ & 58.1 & 72.6 & 19.3 & 8.1 & 1.2 \\
& 0.1 & 0.2 & 0.1 & 0.8 & \pm 0.7 & & & & \\
\hline $\mathrm{Mg}^{2+}$ & $3.9 \pm$ & $16.2 \pm$ & $9.0 \pm$ & $33.1 \pm$ & $58.5 \pm$ & 72.8 & 18.9 & 8.3 & 1.3 \\
& 0.1 & 0.2 & 0.1 & 0.8 & 0.7 & & & & \\
\hline $\mathrm{Ca}^{2+}$ & $4.1 \pm$ & $16.9 \pm$ & $4.7 \pm$ & $21.8 \pm$ & $74.0 \pm$ & 63.3 & 20.3 & 16.4 & 1.1 \\
& 0.1 & 0.2 & 0.1 & 0.6 & 0.6 & & & & \\
\hline $\mathrm{Ca}^{2+} \mathrm{Mg}^{2+}$ & $4.1 \pm$ & $16.9 \pm$ & $4.7 \pm$ & $21.1 \pm$ & $74.9 \pm$ & 63.1 & 20.1 & 16.8 & 1.1 \\
& 0.1 & 0.2 & 0.1 & 0.6 & 0.6 & & & & \\
\hline
\end{tabular}

Recovered from Vinci analysis software using a sum of three discrete exponential decays with 1,8ANS lifetime fixed to $0.28 \mathrm{~ns}$. Phase and modulation errors were set at $\leq 0.2^{\circ}$ and $\leq 0.004$, respectively. $\tau_{0}, \tau_{1}$, and $\tau_{2}$ are lifetimes of the three discrete exponential decays; $f_{0}, f_{1}$ and $f_{2}$ represent exponential decay fractions; $\alpha_{0}, \alpha_{1}$, and $\alpha_{2}$ are pre-exponential factors. 
7.2.2 Consequence of Lys87 to Ala mutation on DREAM interactions with intracellular partners.

Previous studies have shown that KChIP1, a protein with high sequence homology with DREAM, interacts with the T-1 domain of Kv.4 channel through two contact interfaces and this interaction has been demonstrated to result in increased surface expression, alteration of gating properties, and subunit assembly facilitation of $\mathrm{Kv} 4$ channels (Bourdeau, Laplante et al. 2011, Pioletti, Findeisen et al. 2006, Wang, Yan et al. 2007b, Bahring, Dannenberg et al. 2001, Scannevin, Wang et al. 2004). Site 1(residues 1-
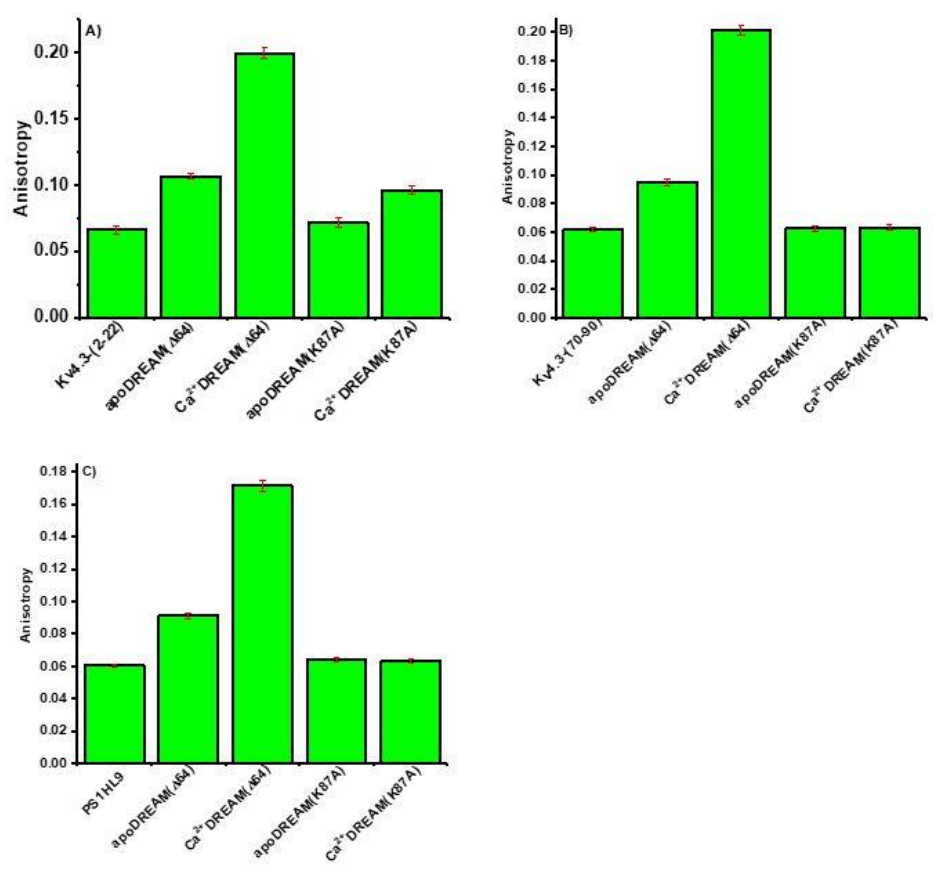

Figure 7.6. Anisotropy change associated with Kv4.3 site 1 (residues 2-22) (A), Kv4.3 site 2 (residues 70-90) (B) and presenilin 1 helix 9 (PS1HL9) (C) binding to $\operatorname{DREAM}(\mathrm{K} 87 \mathrm{~A})$ in the presence and absence of $\mathrm{Ca}^{2+}$.

20) of $\mathrm{T} 1$ domain of $\mathrm{Kv} 4$ channel interacts with $\mathrm{N}$ - and $\mathrm{C}$ - terminal hydrophobic cavity of KChIP1. Site-2 (residue 70-78) of T1 domain of Kv4 channel interacts with KChIP1 through both stacking and salt bridge interaction (Pioletti, Findeisen et al. 2006, Wang, Yan 
et al. 2007a). Our previous result demonstrated that DREAM interacts with site 1 (residues 2-22) and site 2 (residues 70-90) of the T-1 domain of Kv channel. To investigate whether Lys to Ala mutation at position 87 alters DREAM affinity for site 1 and site 2 of T-1 domain of Kv channel, we titrated DREAM(K87A) into FITC-tagged $0.5 \mu \mathrm{M}$ site-1 and site-2 peptide (Azam, Miksovska 2018, Azam, St Luis et al. 2019, Gonzalez, Pham et al. 2014) (Figure 7.6A,B). Anisotropy value did not change upon the addition of saturation concentration of metal-free and $\mathrm{Ca}^{2+}$-bound form of DREAM(K87A) to site 1 and site 2 peptides. As a control experiment, we added the corresponding metal-free and $\mathrm{Ca}^{2+}$-bound form of WTDREAM $(\Delta 64)$ to site 1 and site 2 peptides. In this case, anisotropy value increased significantly, especially in the presence of $\mathrm{Ca}^{2+}$, which is in agreement with our previous study. Altogether, the above results suggest that disrupting interdomain communication between $\mathrm{N}$ - and C- terminal of DREAM by mutating Lys 87 to Ala inhibits DREAM interactions with site 1 and site 2 of $\mathrm{T} 1$ domain of $\mathrm{Kv}$ channel. This result is consistent with the notion of 1,8-ANS lifetime data where DREAM shows a weaker affinity for hydrophobic molecule 1,8-ANS. We postulate that disrupting interdomain communication disrupts the hydrophobic cavities that are involved in the binding of site 1 and site 2 .

Previous studies have shown that DREAM interacts with the carboxyterminal fragment of presenilin- 1 and stimulates the activity of $y$-secretase complex which in turn facilities production of A $\beta 42$, a peptide associated with Alzheimer's disease (Jo, Jang et al. 2005). Previous results of our group have shown that DREAM interacts with presenilin-1 of helix-9 (PS1HL9) in a calcium-dependent manner; DREAM interacts with PS1HL9 through cation $-\pi$ interaction, salt bridge, and hydrophobic interaction. More 
specifically, D450 and D458 of PS1HL9 form a salt bridge with R207 of DREAM; R200 of DREAM forms a cation- $\pi$ interaction with F465 of PS1HL9; and F462 and F465 of PS1HL9 forms hydrophobic interaction with F252 of DREAM (Pham, Miksovska 2016). To investigate whether Lys to Ala mutation at position 87 influences the interaction between DREAM and PS1HL9, we titrated DREAM(K87A), in metal-free form and in the presence of $\mathrm{Ca}^{2+}$, into $0.5 \mu \mathrm{M}$ PS1HL9. Interestingly, our results show that the anisotropy value of PS1HL9 does not increase at all upon the addition of saturation concentration of $\operatorname{DREAM}(\mathrm{K} 87 \mathrm{~A})$, both in apo and $\mathrm{Ca}^{2+}$-bound form. On the other hand, under identical conditions, the anisotropy value of PS1HL9 increases significantly upon the addition of corresponding WTDREAM $(\Delta 64)$, indicating mutation of Lys to Ala at position 87 inhibits

DREAM interactions with PS1HL9. We hypothesize that disrupting interdomain communication allosterically inhibits hydrophobic interaction between DREAM and PS1HL9 as supported by 1,8-ANS lifetime data where DREAM shows a weaker affinity for hydrophobic molecule 1,8-ANS.

\subsection{Summary}

In this study, we have demonstrated that disrupting interdomain communication between $\mathrm{N}$ - and $\mathrm{C}$ - terminal of DREAM by mutating Lys87 to Ala leads to solvent exposure of Trp169 and DREAM-bound 1,8-ANS molecule as supported by emission data; less accessibility of hydrophobic cavity of DREAM for 1,8-ANS association as evident from lifetime data; and inhibition of DREAM interactions with site 1 and site 2 peptide of $\mathrm{T}-1$ domain of $\mathrm{Kv}$ channel and helix 9 of presenilin- 1 based on anisotropy measurements. 
8. IMPACT OF EF-3 OR EF-4 INACTIVATION OF DREAM ON ITS SECONDARY AND TERTIARY STRUCTURES AND INTERACTION WITH T-1 DOMAIN OF KV CHANNEL.

\subsection{Background and significance}

Downstream regulatory element antagonist modulator (DREAM) is a $29 \mathrm{kDa}$ protein that is expressed in the hippocampus of the brain (Carrion, Link et al. 1999). Interestingly, this protein has three different names. It was discovered by three independent research groups and each group named it differently. On the basis of the fact that this protein interacts with presenilin and regulates the activity of $\gamma$ secretase complex, one research group named it calsenilin (Buxbaum, Choi et al. 1998). Later, another group named it as downstream regulatory element antagonist modulator (DREAM) as this protein interacts with downstream regulatory element (DRE) and directly modulate transcription of downstream genes (Carrion, Link et al. 1999). Finally, this protein was renamed as KChIPs as it has high sequence similarity with KChIP-1 and KChIP-2 (An, Bowlby et al. 2000). DREAM is a member of EF-hand superfamily; it also belongs to the neuronal calcium sensor family. DREAM is a multifunctional protein that regulates numerous biological processes, including gene apoptosis, pain sensation, and memory and learning processes (An, Bowlby et al. 2000, Buxbaum, Choi et al. 1998, Carrion, Link et al. 1999).

DREAM has been associated with Alzheimer's disease. It interacts with presenilin-1 (PS1) and presenilin-2 and stimulates the activity of $\gamma$ - secretase complex in a calciumdependent manner and facilitates the generation of amyloid beta plaques in 
Alzheimer's disease (Buxbaum, Choi et al. 1998, Jo, Jang et al. 2005). DREAM also associate with the $\mathrm{T} 1$ domain of potassium channels and play a predominant role in the modulation of fast $\mathrm{I}_{\mathrm{TO}}$ and $\mathrm{I}_{\mathrm{SA}}$ current in the heart and brain, respectively. DREAM binding to the $\mathrm{T} 1$ domain of potassium channels also promotes translocation of the channel to the membrane and alters the gating properties of the channel (An, Bowlby et al. 2000). $\mathrm{Ca}^{2+}$-bound DREAM interacts with $\mathrm{CaM}$ and DREAM:CaM complex formation inhibits DREAM interaction DRE sites and facilitates activation of calcineurin (Gonzalez, Arango et al. 2015, Ramachandran, Craig et al. 2012).

DREAM protein comprises 256 amino acid residues. It also contains four EFhands. The four EF-hands of DREAM differ in terms of metal binding property. EF-hand 1 doesn't bind any metal as the presence of sterically hindered amino acid residue proline in the EF-hand loop (CPXG sequence) destroys metal-binding geometry. EF-2 binds $\mathrm{Mg}^{2+}$ selectively because of the presence of Asp at the $12^{\text {th }}$ position of the EF-hand loop instead of the usual glutamate. It has been demonstrated that $\mathrm{Mg}^{2+}$ binding to EF-2 stabilizes the tertiary structure of DREAM which facilities DNA binding. EF-hand 3 and EF-hand 4 bind $\mathrm{Ca}^{2+}$ preferentially with $\mathrm{K}_{\mathrm{d}}$ of $1-10 \mu \mathrm{M}$ as glutamate is present at the $12^{\text {th }}$ position of the EF-hand loop (Gifford, Walsh et al. 2007, Lusin, Vanarotti et al. 2008, Osawa, Dace et al. 2005, Osawa, Tong et al. 2001). Our recent studies have shown that heavy metals $\mathrm{Pb}^{2+}$ and $\mathrm{Cd}^{2+}$ and lanthanide $\mathrm{Tb}^{3+}$ bind to EF-hand of DREAM with affinity higher than that of $\mathrm{Ca}^{2+}$ (Azam, Miksovska 2018, Azam, St Luis et al. 2019, Gonzalez, Ramos et al. 2016) In the absence of any metal, DREAM exists as a tetramer, but $\mathrm{Ca}^{2+}$ binding triggers conformation changes in the protein that dissociates the tetramer into two dimers 
if the concentration of protein is below $150 \mu \mathrm{M}$. When the concentration of protein is above $200 \mu \mathrm{M}$, DREAM is tetramer in both metal-free forms and $\mathrm{Ca}^{2+}$ bound form (Osawa, Tong et al. 2001).

It is well established that $\mathrm{Ca}^{2+}$ binding causes secondary and tertiary structural rearrangements of the protein and subsequently modulates DREAM interaction with binding partners (Osawa, Dace et al. 2005, Osawa, Tong et al. 2001, Pham, Dhulipala et al. 2015). Our previous studies have also demonstrated that DREAM interacts with hydrophobic molecule 1,8-ANS through hydrophobic cavities of its surface and hydrophobic cavities that bind 1,8-ANS becomes more accessible to 1,8 -ANS in the presence of $\mathrm{Ca}^{2+}$ as evidenced by the fact that emission intensity of DREAM:1,8-ANS is higher in presence of $\mathrm{Ca}^{2+}$ compared with corresponding metal-free form (Gonzalez, Miksovska 2014). But the underlying mechanism of $\mathrm{Ca}^{2+}$-triggered structural rearrangements of DREAM and modulation of interactions with binding partners is not resolved yet. In this study, we mutated glutamate residue at $12^{\text {th }}$ position of EF-3 (DREAM(E186Q)) or EF-4 (DREAM(E234Q)) to glutamine for making these EF-hands incapable of binding $\mathrm{Ca}^{2+}$ and investigated whether mutations impact $\mathrm{Ca}^{2+}$-triggered secondary and tertiary structural rearrangements of the protein and whether mutations impact $\mathrm{Ca}^{2+}$-induced modulation of DREAM interaction with hydrophobic molecule 1,8 ANS and T-1 domain of Kv channel. 


\subsection{Results and discussion}

8.2.1 Consequence of EF-3 or EF-4 inactivation of DREAM on Its Secondary and Tertiary Structure

The previous study demonstrated that $\mathrm{Ca}^{2+}$ binding triggers tertiary structural rearrangements of DREAM and emission intensity decreases upon $\mathrm{Ca}^{2+}$ binding to $\operatorname{DREAM}(\Delta 64)$ and C-terminal of the DREAM, DREAM $(\Delta 160)($ Gonzalez, Ramos et al. 2016, Pham, Dhulipala et al. 2015). It is also well established from the previous study that $\mathrm{Ca}^{2+}$ binds to EF-3 and EF-4 at the C-terminal of the protein. As a control experiment, we
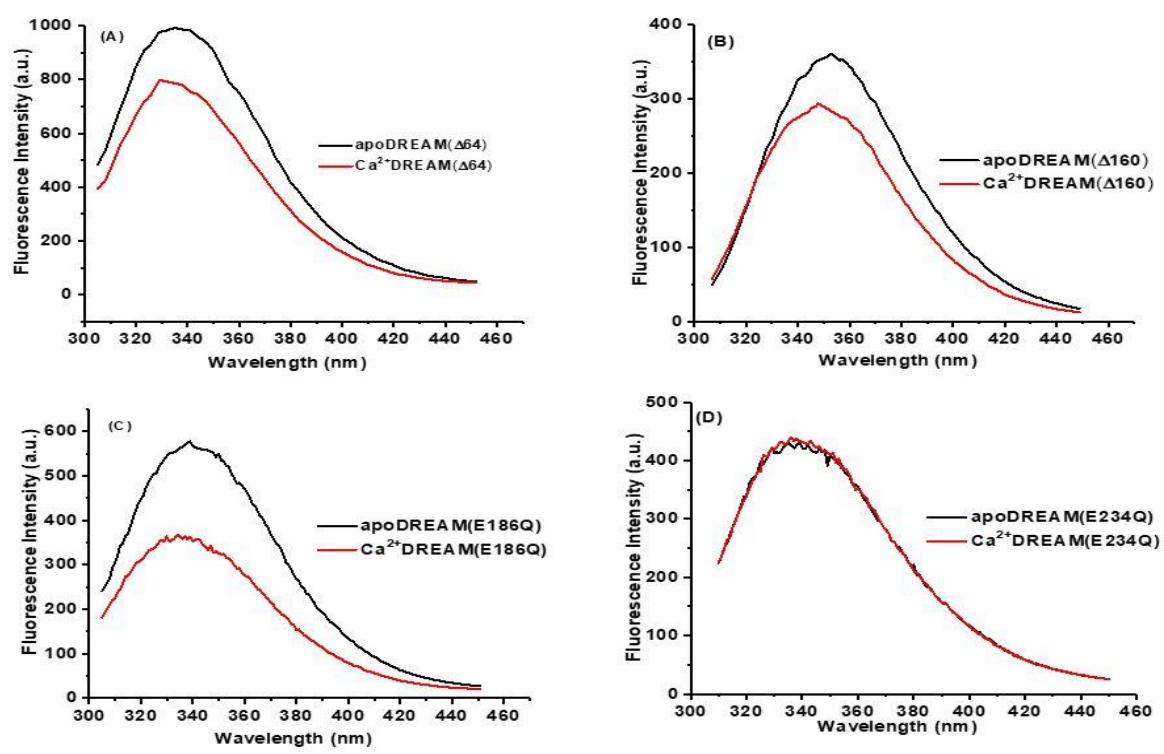

Figure 8.1. Steady-state Trp emission spectra of $20 \mu \mathrm{M} \operatorname{DREAM}(\Delta 64)$, $\operatorname{DREAM}(\Delta 160), \operatorname{DREAM}(\mathrm{E} 186 \mathrm{Q})$, and DREAM(E234Q) in the absence and presence of $1 \mathrm{mM} \mathrm{Ca}^{2+}$; excited at $295 \mathrm{~nm}$.

measured emission spectra of $\operatorname{DREAM}(\Delta 64)$ and $\operatorname{DREAM}(\Delta 160)$ both in the presence and absence of $\mathrm{Ca}^{2+}$ (Figure 8.1A,B). Emission intensity decrease upon $\mathrm{Ca}^{2+}$ binding to both $\operatorname{DREAM}(\Delta 64)$ and $\operatorname{DREAM}(\Delta 160)$, which is in agreement with previous studies. Then we measured emission spectra of the DREAM(E186Q), a DREAM construct that is not 
expected to bind $\mathrm{Ca}^{2+}$ and other metals at EF-3 due to glutamate to glutamine mutation at position 186, and DREAM(E234Q), a DREAM construct that is not anticipated to bind $\mathrm{Ca}^{2+}$ at EF-4 due to glutamate to glutamine mutation at position 234 (Figure 8.1C,D). The emission intensity decreases upon $\mathrm{Ca}^{2+}$ binding to DREAM(E186Q), a DREAM construct with EF-3 inactive, but EF-4 active, suggesting $\mathrm{Ca}^{2+}$ binding to EF-4 could trigger structural rearrangement that causes the decrease in emission intensity. Interestingly, emission spectrum of DREAM(E234Q), DREAM construct with EF-4 inactive, but EF-3 inactive, is the same in the presence and absence of $\mathrm{Ca}^{2+}$, indicating binding of $\mathrm{Ca}^{2+}$ at $\mathrm{EF}$ 4 might not cause $\mathrm{Ca}^{2+}$-triggered tertiary structural rearrangement. The emission study is supported by Trp lifetime study (Figure 8.2A,B and Table 8.1). The lifetime of Trp 169 is sensitive to structural rearrangement. The average lifetime of Trp169 in DREAM $(\Delta 64)$ decreases from $3.8 \mathrm{~ns}$ to $3.1 \mathrm{~ns}$ upon $\mathrm{Ca}^{2+}$ binding. Like DREAM $(\Delta 64)$, the average lifetime of Trp169 in DREAM(E186Q) decreases from 4.2 ns to 3.6 ns upon $\mathrm{Ca}^{2+}$ addition, but the average lifetime of Trp169 in DREAM(E234Q) remains same (4.3 ns) upon $\mathrm{Ca}^{2+}$ addition, which bolster finding of Trp emission study. To get insight about $\mathrm{Ca}^{2+}$ triggered secondary
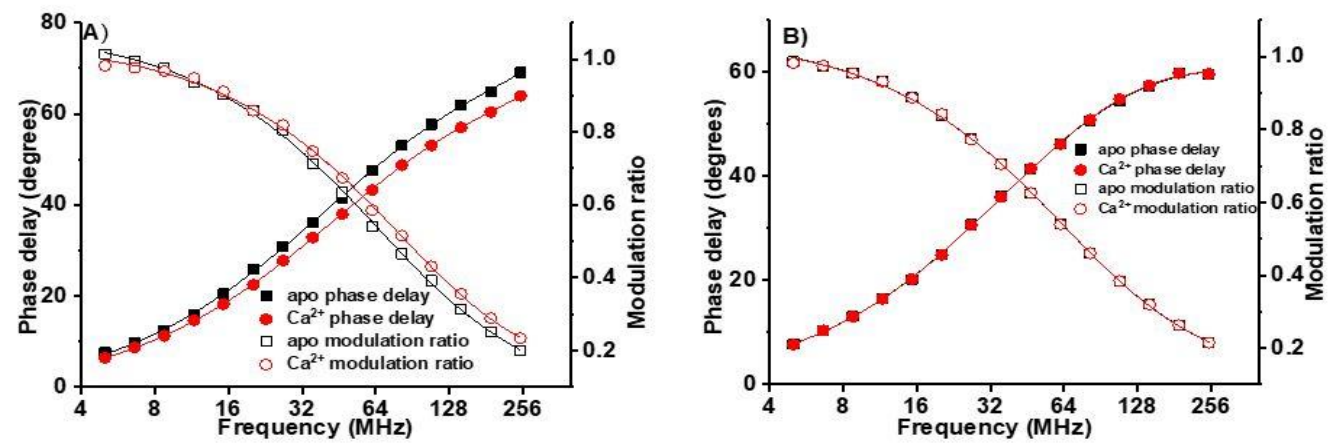

Figure 8.2. Frequency-domain intensity decay of $20 \mu \mathrm{M}$ DREAM(E186Q) (A) and DREAM(E234Q) (B) in apo (square) and $\mathrm{Ca}^{2+}$ (circle) bound form. Solid symbols represent phase delay; empty symbols represent modulation ratio. Solid lines represent fitting of the data using a sum of three exponential decays model. 
structural rearrangement we measured far-UV CD spectra of all four DREAM constructs (Figure 8.3A-D). We calculated percentage of $\alpha$-helix using online program K2D3 and results show that $\mathrm{Ca}^{2+}$ binding causes $\sim 6$ increase in $\alpha$-helical content in $\operatorname{DREAM}(\Delta 64)$ and DREAM(E186Q) and 10\% increase in $\alpha$-helical content of $\operatorname{DREAM}(\Delta 160)$ but $\alpha$ helical content of DREAM(E234Q) remains same upon $\mathrm{Ca}^{2+}$ addition. Above far-UV CD data is in agreement with the notion of Trp emission and Trp lifetime data.
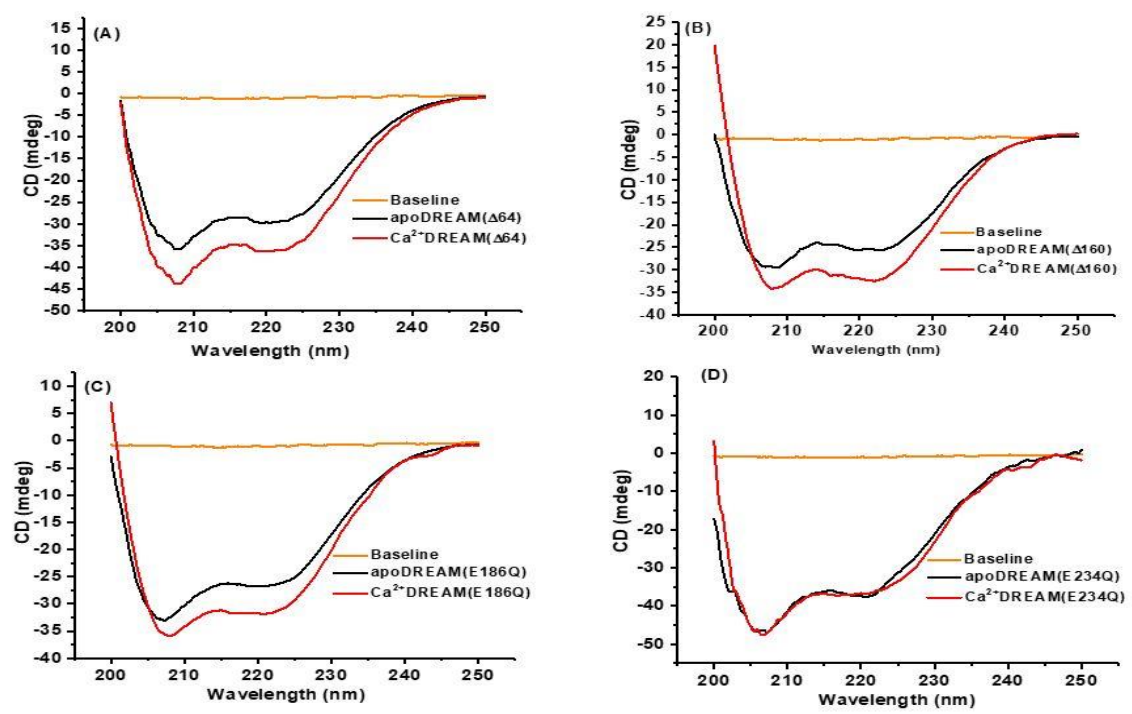

Figure 8.3. Far-UV CD spectra of DREAM $(\Delta 64)(\mathrm{A}), \operatorname{DREAM}(\Delta 160)$ (B), DREAM(E186Q) (C), and DREAM(E234Q) (D) in the absence and presence of $1 \mathrm{mM} \mathrm{Ca}{ }^{2+}$. 
Table 8.1: Fluorescence decay parameters of DREAM constructs in the presence and absence of metals.

\begin{tabular}{|c|c|c|c|c|c|c|c|c|c|c|c|}
\hline Samples & $\begin{array}{l}\tau_{1} \\
(\mathrm{~ns})\end{array}$ & $\begin{array}{l}\tau_{2} \\
\text { (ns) }\end{array}$ & $\begin{array}{l}\tau_{3} \\
(\mathrm{~ns})\end{array}$ & $\begin{array}{l}\mathrm{f}_{1} \\
(\%)\end{array}$ & $\begin{array}{l}\mathrm{f}_{2} \\
(\%)\end{array}$ & $\begin{array}{l}\mathrm{f}_{3} \\
(\%)\end{array}$ & $\begin{array}{l}\alpha_{1} \\
(\%)\end{array}$ & $\begin{array}{l}\alpha_{2} \\
(\%)\end{array}$ & $\begin{array}{l}\alpha_{3} \\
(\%)\end{array}$ & $\begin{array}{l}<\tau> \\
(\mathrm{ns})\end{array}$ & $\chi^{2}$ \\
\hline $\begin{array}{l}\text { ApoDREAM } \\
(\Delta 64)\end{array}$ & $\begin{array}{l}0.24 \pm \\
0.03\end{array}$ & $\begin{array}{l}2.5 \pm \\
0.1\end{array}$ & $\begin{array}{l}7.1 \pm \\
0.3\end{array}$ & $\begin{array}{l}17.3 \pm \\
0.8\end{array}$ & $\begin{array}{l}45.4 \pm \\
3.0\end{array}$ & $\begin{array}{l}37.3 \pm \\
0.3\end{array}$ & 75.1 & 19.2 & 5.9 & 3.8 & 1.5 \\
\hline $\begin{array}{l}\mathrm{Ca}^{2+} \text { DREAM } \\
(\Delta 64)\end{array}$ & $\begin{array}{l}0.16 \pm \\
0.02\end{array}$ & $\begin{array}{l}1.9 \pm \\
0.1\end{array}$ & $\begin{array}{l}6.3 \pm \\
0.1\end{array}$ & $\begin{array}{l}22.0 \pm \\
0.9\end{array}$ & $\begin{array}{l}37.5 \pm \\
2.0\end{array}$ & $\begin{array}{l}40.5 \pm \\
2.0\end{array}$ & 84.5 & 12.1 & 3.3 & 3.1 & 0.9 \\
\hline $\begin{array}{l}\text { apoDREAM( } \\
\text { E186Q) }\end{array}$ & $\begin{array}{l}0.56 \pm \\
0.06\end{array}$ & $\begin{array}{l}2.5 \pm \\
0.2\end{array}$ & $\begin{array}{l}6.4 \pm \\
0.3\end{array}$ & $\begin{array}{l}7.5 \pm \\
1.0\end{array}$ & $\begin{array}{l}44.4 \pm \\
3.0\end{array}$ & $\begin{array}{l}49.1 \pm \\
4.0\end{array}$ & 34.2 & 46.1 & 19.6 & 4.2 & 1.6 \\
\hline $\begin{array}{l}\mathrm{Ca}^{2+} \mathrm{DREAM} \\
(\mathrm{E} 186 \mathrm{Q})\end{array}$ & $\begin{array}{l}0.48 \pm \\
0.04\end{array}$ & $\begin{array}{l}2.4 \pm \\
0.1\end{array}$ & $\begin{array}{l}6.2 \pm \\
0.3\end{array}$ & $\begin{array}{l}10.9 \pm \\
1.01\end{array}$ & $\begin{array}{l}49.4 \pm \\
3.0 \\
\end{array}$ & $\begin{array}{l}39.4 \pm \\
4.0 \\
\end{array}$ & 45.3 & 41.9 & 12.8 & 3.6 & 1.4 \\
\hline $\begin{array}{l}\text { apoDREAM( } \\
\text { E234Q) }\end{array}$ & $\begin{array}{l}0.27 \pm \\
0.03\end{array}$ & $\begin{array}{l}2.6 \pm \\
0.2\end{array}$ & $\begin{array}{l}6.9 \pm \\
0.3\end{array}$ & $\begin{array}{l}8.6 \pm \\
0.5\end{array}$ & $\begin{array}{l}46.6 \pm \\
3.0\end{array}$ & $\begin{array}{l}44.7 \pm \\
3.0\end{array}$ & 56.2 & 32.2 & 11.5 & 4.3 & 1.5 \\
\hline $\begin{array}{l}\mathrm{Ca}^{2+} \mathrm{DREAM} \\
(\mathrm{E} 234 \mathrm{Q})\end{array}$ & $\begin{array}{l}0.27 \pm \\
0.03\end{array}$ & $\begin{array}{l}2.6 \pm \\
0.1\end{array}$ & $\begin{array}{l}6.9 \pm \\
0.3\end{array}$ & $\begin{array}{l}8.5 \pm \\
8.4\end{array}$ & $\begin{array}{l}48.4 \pm \\
3.0\end{array}$ & $\begin{array}{l}42.9 \pm \\
3.0\end{array}$ & 56.5 & 32.6 & 10.8 & 4.3 & 1.9 \\
\hline
\end{tabular}

Recovered from Vinci analysis software using a sum of three discrete exponential decays. Phase and modulation errors were set at $\leq 0.2^{\circ}$ and $\leq 0.004$, respectively. $\tau_{1}, \tau_{2}$, and $\tau_{3}$ are lifetimes of the three discrete exponential decays; $\mathrm{f}_{1}, \mathrm{f}_{2}$ and $\mathrm{f}_{3}$ represent exponential decay fractions; $\alpha_{1}, \alpha_{2}$, and $\alpha_{3}$ are pre-exponential factors. 
8.2.2 Effects of EF-3 or EF-4 inactivation of DREAM on Its interaction with hydrophobic molecule 1,8-ANS.

Hydrophobic molecule 1,8-ANS has been widely used for probing hydrophobicity of protein surface as 1,8-ANS is almost non-fluorescence in solution but fluorescence intensity increases by many-folds upon association with hydrophobic patches of protein surface (Cardamone, Puri 1992). Our previous study has demonstrated that DREAM is capable of binding 1,8-ANS in metal-free form but $\mathrm{Ca}^{2+}$ association to
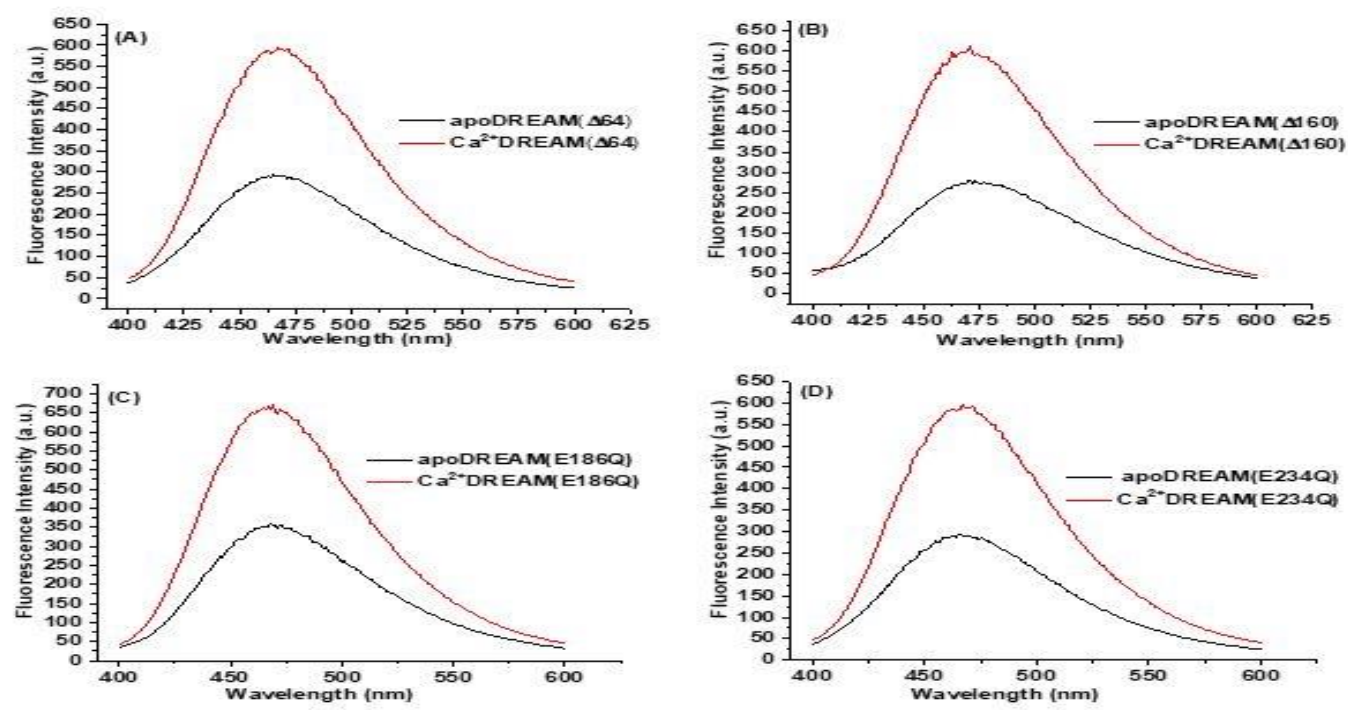

Figure 8.4. Fluorescence emission of $\operatorname{DREAM}(\Delta 64): 1,8-\mathrm{ANS}$ (A), $\operatorname{DREAM}(\Delta 160): 1,8-\mathrm{ANS} \quad$ (B), DREAM(E186):1,8-ANS $\quad(\mathrm{C}), \quad$ and DREAM(E234):1,8-ANS complexes in metal-free form and in the presence of $1 \mathrm{mM}$ $\mathrm{Ca}^{2+}$. Condition: $20 \mu \mathrm{M}$ DREAM constructs, $20 \mu \mathrm{M}$ 1,8-ANS, excited at $350 \mathrm{~nm}$. DREAM increases its affinity for 1,8-ANS. In this case, we investigated whether inactivation of either EF-3 or EF-4 impacts 1,8-ANS (Gonzalez, Miksovska 2014). As a control, we measured emission intensity of $\operatorname{DREAM}(\Delta 64): 1,8-\mathrm{ANS}$ and $\operatorname{DREAM}(\Delta 160): 1,8$-ANS complexes (Figure 8.4A,B) both in the presence $\mathrm{Ca}^{2+}$ and in metal-free form and the result is consistent with previous studies; that is, the emission 
intensity of 1,8-ANS increases significantly upon binding to these protein constructs in metal-free form and the emission intensity of both DREAM( $(664): 1,8$-ANS and $\operatorname{DREAM}(\Delta 160): 1,8$-ANS complexes are higher in the presence of $\mathrm{Ca}^{2+}$ compared with corresponding metal-free form. Like above complexes, the emission intensity of DREAM(E186Q):1,8-ANS and DREAM(E234Q):1,8-ANS complexes (Figure 8.4C,D) increase significantly upon $\mathrm{Ca}^{2+}$ association compared with corresponding metal-free form, suggesting binding of one $\mathrm{Ca}^{2+}$ in either EF-3 or EF-4 is good enough for increasing the accessibility for 1,8-ANS binding site.

To support the above emission studies, we also measured the lifetime of the above complexes in metal-free form and in the presence of $\mathrm{Ca}^{2+}$ (Figure 8.5A,B, Table 8.2). Previously we showed that 1,8-ANS binds to two distinct binding sites on the DREAM surface (Gonzalez, Miksovska 2014). Modulation-phase data for all complexes were best
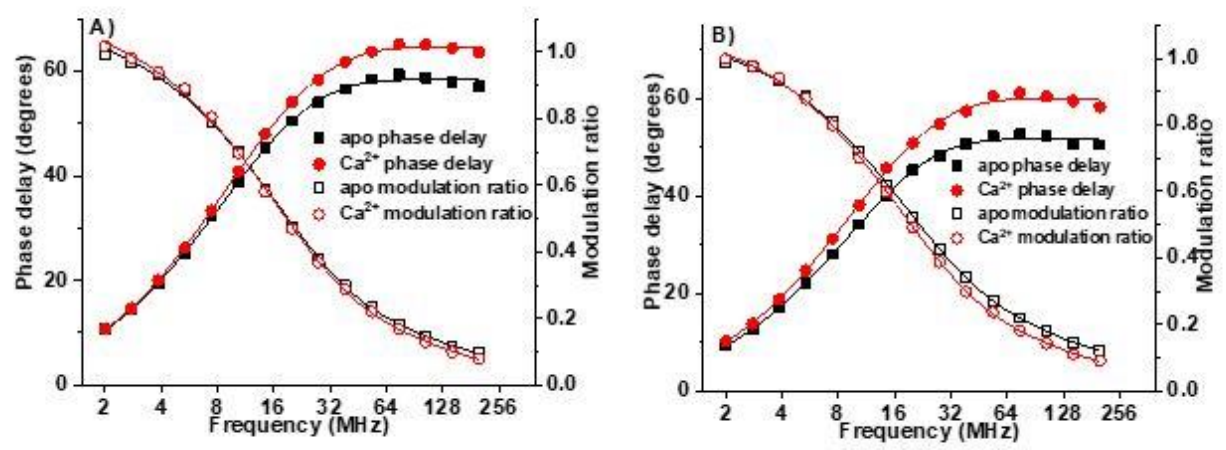

Figure 8.5. Frequency-domain intensity decay of DREAM(E186Q):1,8-ANS (A) and DREAM(E234Q):1,8-ANS (B) in apo (square) and $\mathrm{Ca}^{2+}$-bound form (circle). Solid symbols represent phase delay; empty symbols represent modulation ratio. Solid lines represent fitting of the data using a sum of three exponential decays model. 
fitted by a three exponential decay model with 1,8-ANS lifetime fixed to 0.28 ns. Faster lifetime $\tau_{1}$ could be attributed to 1,8 -ANS binding to the partially solvent-exposed binding site on DREAM, whereas longer lifetime $\tau_{2}$ can be assigned to 1,8-ANS binding to nonpolar solvent restricted binding site on DREAM. Inactivating EF-3 or EF-4 did not significantly alter individual lifetimes, but $\alpha_{1}$ and $\alpha_{2}$ values are smaller in DREAM(E186Q):1,8-ANS and DREAM(E234Q):1,8-ANS complexes both in the presence of $\mathrm{Ca}^{2+}$ and in metal-free form compared with corresponding $\operatorname{DREAM}(\Delta 64): 1,8$ ANS (Table 8.2), suggesting that inactivating either EF-3 or EF-4 decreases DREAM affinity for 1,8-ANS. Interestingly, the value of $\alpha_{2}$ did not change significantly upon $\mathrm{Ca}^{2+}$ binding to DREAM(E186Q):1,8-ANS and DREAM(E234Q):1,8-ANS complexes, but the value of $\alpha_{2}$ increased by 2 -folds upon $\mathrm{Ca}^{2+}$ binding to metal-free $\operatorname{DREAM}(\Delta 64): 1,8$-ANS (Table 8.2). It is possible that binding of one $\mathrm{Ca}^{2+}$ to DREAM(E186Q):1,8-ANS and DREAM(E234Q):1,8-ANS complexes may not fully expose the hydrophobic cavities that bind 1,8-ANS. 
Table 8.2: Fluorescence decay parameters of DREAM:1,8-ANS complexes.

\begin{tabular}{|c|c|c|c|c|c|c|c|c|}
\hline DREAM:1,8-ANS & $\begin{array}{l}\tau_{1} \\
(\mathrm{~ns})\end{array}$ & $\begin{array}{l}\tau_{2} \\
(\mathrm{~ns})\end{array}$ & $\begin{array}{l}\mathrm{f}_{1} \\
(\%)\end{array}$ & $\begin{array}{l}\mathrm{f}_{2} \\
(\%)\end{array}$ & $\begin{array}{l}\alpha_{0} \\
(\%)\end{array}$ & $\begin{array}{l}\alpha_{1} \\
(\%)\end{array}$ & $\begin{array}{l}\alpha_{2} \\
(\%)\end{array}$ & $\chi^{2}$ \\
\hline $\operatorname{apoDREAM}(\Delta 64)$ & $4.1 \pm 0.1$ & $\begin{array}{l}16.9 \pm \\
0.2\end{array}$ & $\begin{array}{l}15.4 \pm \\
0.5\end{array}$ & $\begin{array}{l}83.0 \pm \\
1.0\end{array}$ & 62.6 & 15.5 & 21.9 & 0.9 \\
\hline $\mathrm{Ca}^{2+} \operatorname{DREAM}(\Delta 64)$ & $4.3 \pm 0.2$ & $\begin{array}{l}18.0 \pm \\
0.2\end{array}$ & $9.7 \pm 0.6$ & $\begin{array}{l}88.9 \pm \\
0.5\end{array}$ & 40.4 & 16.9 & 42.7 & 0.9 \\
\hline apoDREAM(E186Q) & $3.5 \pm 0.1$ & $\begin{array}{l}17.2 \pm \\
0.1\end{array}$ & $\begin{array}{l}12.3 \pm \\
0.4\end{array}$ & $\begin{array}{l}83.3 \pm \\
0.4\end{array}$ & 68.9 & 13.0 & 18.1 & 1.5 \\
\hline $\mathrm{Ca}^{2+} \mathrm{DREAM}(\mathrm{E} 186 \mathrm{Q})$ & $3.7 \pm 0.1$ & $\begin{array}{l}17.0 \pm \\
0.1\end{array}$ & $\begin{array}{l}10.1 \pm \\
0.8\end{array}$ & $\begin{array}{l}88.6 \pm \\
0.5\end{array}$ & 60.7 & 13.5 & 25.8 & 1.2 \\
\hline apoDREAM(E234Q) & $3.6 \pm 0.1$ & $\begin{array}{l}16.1 \pm \\
0.1\end{array}$ & $\begin{array}{l}16.4 \pm \\
0.5\end{array}$ & $\begin{array}{l}75.3 \pm \\
0.5\end{array}$ & 76.1 & 11.8 & 12.1 & 1.8 \\
\hline $\mathrm{Ca}^{2+} \mathrm{DREAM}(\mathrm{E} 234 \mathrm{Q})$ & $3.9 \pm 0.1$ & $\begin{array}{l}16.7 \pm \\
0.1\end{array}$ & $\begin{array}{l}13.2 \pm \\
0.6\end{array}$ & $\begin{array}{l}82.7 \pm \\
0.6\end{array}$ & 67.7 & 13.1 & 19.2 & 1.7 \\
\hline \multicolumn{9}{|c|}{$\begin{array}{l}\text { Recovered from Vinci analysis software using a sum of three discrete exponential decays with } 1,8 \text {-ANS lifetime } \\
\text { fixed to } 0.28 \mathrm{~ns} \text {. Phase and modulation errors were set at } \leq 0.2^{\circ} \text { and } \leq 0.004 \text {, respectively. } \tau_{0}, \tau_{1} \text {, and } \tau_{2} \text { are } \\
\text { lifetimes of the three discrete exponential decays; } f_{0}, f_{1} \text { and } f_{2} \text { represent exponential decay fractions; } \alpha_{0}, \alpha_{1} \text {, and } \alpha_{2} \\
\text { are pre-exponential factors. }\end{array}$} \\
\hline
\end{tabular}


8.2.3 Impact of EF-3 or EF-4 Inactivation of DREAM on Its Interaction with T-1 Domain of Kv Channel.

Previous studies have demonstrated that KChIP1, a protein with high sequence similarity with DREAM, interacts with the T-1 domain of Kv.4 channel through two contact interfaces and this interaction has been shown to cause modulation of gating properties, enhancement of surface expression, and facilitation of subunit assemblies of Kv4 channels (Bourdeau, Laplante et al. 2011, Pioletti, Findeisen et al. 2006, Wang, Yan et al. 2007b, Bahring, Dannenberg et al. 2001, Scannevin, Wang et al. 2004). Residues 1-20 (site-1) of the $\mathrm{T} 1$ domain of $\mathrm{Kv} 4$ channel interacts with $\mathrm{N}$ - and $\mathrm{C}$ - terminal hydrophobic patches of KChIP1. Residue 70-78 (site-2) of the T1 domain of Kv4 channel interacts with KChIP1 through both stacking and salt bridge interaction (Pioletti, Findeisen et al. 2006, Wang, Yan et al. 2007a). Our previous result showed that DREAM interacts with site 1 (residues 2-22) and site 2 (residues 70-90) of T-1 domain of the Kv channel (Azam, Miksovska 2018, Azam, St Luis et al. 2019, Gonzalez, Pham et al. 2014). To investigate whether EF-3 or EF-4 inactivation of DREAM modulates DREAM affinity for site 1 and site 2 of the T-1 domain of Kv channel, we titrated DREAM(E186Q) and DREAM(E234Q) into FITCtagged $0.5 \mu \mathrm{M}$ site-1 and site-2 peptide (Figure 8.6A,B). Anisotropy value did not increase upon the addition of saturation concentration DREAM(E186) or DREAM(E234Q) to site 1 peptide both in the presence and absence of $\mathrm{Ca}^{2+}$. As a control experiment, we added corresponding wild-type $\operatorname{DREAM}(\Delta 64)$ to site peptides both in the presence and absence of $\mathrm{Ca}^{2+}$. In this case, anisotropy value increased significantly, especially in the presence of $\mathrm{Ca}^{2+}$, which is similar to our previous study. Site 1 binds to N-and C-terminal hydrophobic 

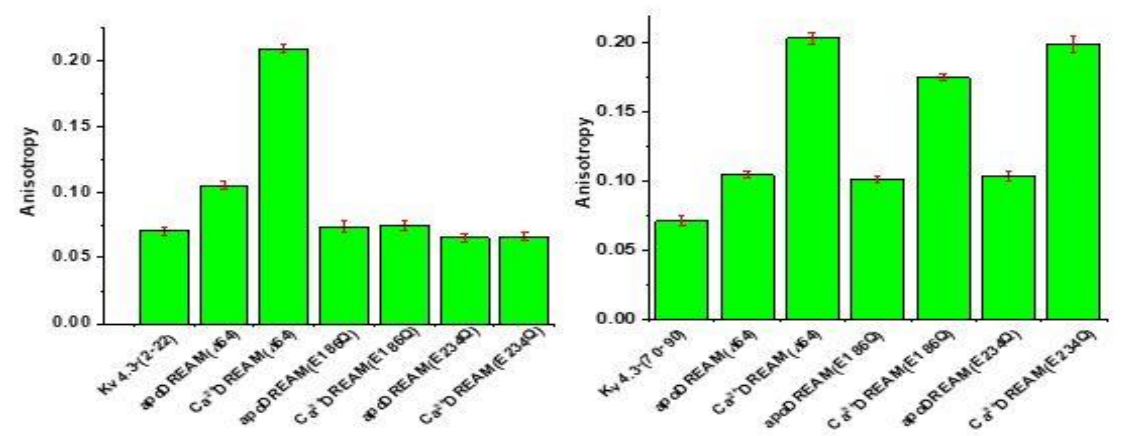

Figure 8.6. Anisotropy change associated with Kv4.3 site-1 (residues 2-22) (A) and Kv4.3 site- 2 (residues 70-90) (B) association with DREAM(E186Q) and $\operatorname{DREAM}(\mathrm{E} 234 \mathrm{Q})$ in the presence and absence of $\mathrm{Ca}^{2+}$.

patches on DREAM surface. We postulate that inactivating either EF-3 or EF-4 disturbs big hydrophobic patches in the C-terminal of DREAM and subsequently prevents interaction between DREAM and site-1. DREAM(E186Q) and DREAM(E234Q) addition to site-2 show an increase in anisotropy, which is similar to wild-type $\operatorname{DREAM}(\Delta 64)$ addition site-2, suggesting inactivating either EF-3 or EF-4 may not modulate DREAM interaction with site- 2 of T-1 domain of Kv4 channels. The site- 2 binding site is located at the N-terminus of DREAM. Inactivating EF-3 or EF-4 at the C-terminus of DREAM may likely not have impact on site-2 binding at the N-terminus.

\subsection{Summary}

In this study, we have shown that $\mathrm{Ca}^{2+}$ binding to EF-4 triggers secondary and tertiary structural rearrangements of DREAM based on CD-data, Trp emission, and Trp lifetime data. Inactivation either EF-3 or EF-4 decreases DREAM affinity for hydrophobic molecule 1,8-ANS based on 1,8-ANS lifetime data. Lastly, inactivating EF-3 prevents DREAM interaction with site- 1 of the $\mathrm{T}-1$ domain of $\mathrm{K}^{+}$channel. 


\section{CONCLUSIONS}

Exposure to toxic metals such as $\mathrm{Cd}^{2+}$ and $\mathrm{Pb}^{2+}$ has been associated with learning difficulties, IQ drop, and cognitive deficiencies in children and different types of cancers and neurodegenerative diseases, but the molecular mechanism through which these metals induces toxicity remains unexplored. DREAM is expressed in the hippocampus, the part of the brain regulates memory consolidation and learning. $\mathrm{Cd}^{2+}$ and $\mathrm{Pb}^{2+}$ have been shown to strongly impact the hippocampus region. These metals also have been shown to bind to EF-hand of calmodulin (CaM) and troponin $\mathrm{C}(\mathrm{TnC})$ with a higher affinity that physiological ligand $\mathrm{Ca}^{2+}$. Binding of these toxic metals modulates CaM interactions with downstream partners, which is considered one of the possible mechanisms of $\mathrm{Pb}^{2+}$-induced neurotoxicity. Considering the facts that $\mathrm{Pb}^{2+}$ and $\mathrm{Cd}^{2+}$ impact hippocampus region and bind to EFhand protein and DREAM is an EF-hand protein and expressed in the hippocampus, we investigated whether $\mathrm{Cd}^{2+}$ and $\mathrm{Pb}^{2+}$ bind to DREAM. In chapter 3 , we demonstrated the binding of $\mathrm{Pb}^{2+}$ to $\mathrm{DREAM}$ protein for the first time. We showed that $\mathrm{Pb}^{2+}$ binds to EF-hand on DREAM with a higher affinity than $\mathrm{Ca}^{2+}$ and capable of displacing $\mathrm{Ca}^{2+}$. Using fluorescence titration, we determined the dissociation constant for $\mathrm{Pb}^{2+}$ binding to DREAM to be $20 \pm 2 \mathrm{nM} . \mathrm{Pb}^{2+}$ association induces the secondary and tertiary structural rearrangement of this protein that is analogous to $\mathrm{Ca}^{2+}$ association as evidenced from Trp emission and circular dichroism data. $\mathrm{Pb}^{2+}$ binding exposes the hydrophobic patches of the DREAM and subsequently facilitates the binding of hydrophobic molecule 1,8-ANS. From the fluorescence titration, we determined that 1,8-ANS associates to DREAM with a 2-fold higher 
affinity in the presence of $\mathrm{Pb}^{2+}$ compared with the metal-free form. Isothermal titration calorimetry experiments provided thermodynamics insight of the $\mathrm{Pb}^{2+}$ association to DREAM. ITC data reveal two $\mathrm{Pb}^{2+}$ ions bind to DREAM protein with dissociation constants $26 \mathrm{nM}$ and $86 \mathrm{nM}$, respectively and binding of $\mathrm{Pb}^{2+}$ to DREAM is entropydriven as evidenced by positive enthalpy and positive entropy change. Most interestingly, we demonstrated that the $\mathrm{Pb}^{2+}$ association alters DREAM interaction with intracellular partners based on anisotropic titration of DREAM protein with peptide-based model systems. More specifically, we determined that DREAM interacts with $\mathrm{Kv} 4.3(2-22)$ and $\mathrm{Kv} 4.3(70-90)$ peptides of T-1 domain of $\mathrm{Kv}$ channel with about 7-fold and 50-fold higher affinity, respectively, in the presence of $\mathrm{Pb}^{2+}$, compared with the corresponding apo-form. Considering the implication of $\mathrm{Kv}$ channels in hippocampus-dependent memory and learning processes, $\mathrm{Kv}$ channel:KChIP complexes may rerpesent molecular target contributing to $\mathrm{Pb}^{2+}$ induced memory and learning deficiencies. Also, DREAM interacts with helix 9 of presenilin-1 (PS1HL9) with about 70 times higher affinity than correspoding metal-free form. Several studies demonstrated that an early life exposure to $\mathrm{Pb}^{2+}$ promotes developmental reprogramming of APP gene expression, leading to an augmented expression of amyloid precursor protein during adulthood. The interaction between $\mathrm{Pb}^{2+}$-bound DREAM and PS1HL9 observed here suggest that elevated concentrations of $\mathrm{Pb}^{2+}$ may directly impact the proteolytic processing of the $\beta$-amyloid precursor protein. Considering high sequence identity of the $\mathrm{C}$-terminal domain of neuronal calcium sensors and significant amount of $\mathrm{Pb}^{2+}$ in the brain $(0.52-4$ ppm) due to occupational and environmental exposure to the $\mathrm{Pb}^{2+}$, especially in the 
hippocampus, other members of NCS family are likely to bind $\mathrm{Pb}^{2+}$ with higher affinity than that for $\mathrm{Ca}^{2+}$ and be involved in $\mathrm{Pb}^{2+}$-induced neurotoxicity.

In chapter 4 , we demonstrated $\mathrm{Cd}^{2+}$ binding to DREAM. We show $\mathrm{Cd}^{2+}$ binding triggers secondary and tertiary structural rearrangement of the DREAM based on Trp emission and CD spectra. From the emission and CD spectra of DREAM protein in which either EF-3 or EF-4 is inactivated, we found that $\mathrm{Cd}^{2+}$ binding at EF-4 could trigger structural rearrangement of DREAM. We determined that $\mathrm{Cd}^{2+}$ binds to DREAM with a dissociation constant $89 \pm 10 \mathrm{nM}$, which is about 5-times weaker than $\mathrm{Pb}^{2+}$ binding to DREAM. Interestingly, although $\mathrm{Cd}^{2+}$ ion has similar ionic radii and charge/radius ratio values as $\mathrm{Ca}^{2+}$, the association of $\mathrm{Cd}^{2+}$ to EF-3 and EF-4 triggers a conformational transition that is somewhat restricted compared to that determined for the binding of the physiological ligand, $\mathrm{Ca}^{2+}$. This is substantiated by 1,8 -ANS emission data as well as lower affinity constants for binding of peptides that mimic physiological effector proteins presenilin and $\mathrm{T} 1$ domain of $\mathrm{Kv}$ channels. Isothermal titration calorimetry data unveiled that $\mathrm{Cd}^{2+}$ association with DREAM is accompanied with a positive change in entropy and enthalpy, suggesting the reaction is entropy-driven. Considering high sequence similarity of DREAM with other NCS proteins, $\mathrm{Cd}^{2+}$ could bind to other NCS proteins and these interactions could provide insight into the molecular basis of neurotoxicity.

In chapter 5, we determined $\mathrm{Li}^{+}$binding to DREAM. Although $\mathrm{Li}^{+}$-based compounds have been used as a first line therapy for the treatment of severe mental disorders for more than six decades and have a wide range of applications in psychopharmacology, the molecular mechanism of $\mathrm{Li}^{+}$action remains largely unknown. Inhibitions of inositol 
monophosphatase (IMPase) and glycogen synthase kinase-3 beta (GSK-3 $\beta$ ) were proposed as molecular mechanisms of $\mathrm{Li}^{+}$action with $\mathrm{Li}^{+}$inhibiting IMPase by displacing the native cofactor $\mathrm{Mg}^{2+}$. Recent in vivo and in cellular studies demonstrated that chronic $\mathrm{Li}^{+}$ administration provides a protective effect against neurodegenerative diseases including Alzheimer, Parkinson and Huntington disease. However, the exact mechanism through which $\mathrm{Li}^{+}$exerts its therapeutic action remains an active area of research. We report that neuroprotective agent $\mathrm{Li}^{+}$binds to DREAM with an equilibrium dissociation constant of $34 \pm 4 \mu \mathrm{M}$ and impacts DREAM structural and dynamic properties in a similar manner as observed for its physiological ligand, $\mathrm{Ca}^{2+}$. The results of fluorescence spectroscopy and molecular dynamics are consistent with $\mathrm{Li}^{+}$binding at the chelating loop of the EF-hands. In the $\mathrm{Li}^{+}$bound form, DREAM association to peptides mimicking DREAM binding sites in voltage-gated potassium channel is enhanced compared to the apoprotein, whereas DREAM affinity for presenilin binding site, helix-9, is impeded. The reduced affinity of $\mathrm{Li}^{+}$DREAM for helix-9 compared with $\mathrm{Ca}^{2+}$ DREAM is in line with in vivo results showing that $\mathrm{Li}^{+}$administration decreases levels of soluble $\mathrm{A} \beta_{42}$ in transgenic mouse model of Alzheimer's disease. These results suggest that DREAM and possibly other members of neuronal calcium sensor family belong to $\mathrm{Li}^{+}$intracellular targets and interactions between $\mathrm{Li}^{+}$and NCS provide a molecular basis for $\mathrm{Li}^{+}$neuroprotective action.

In chapter 6, we investigated whether toxic metals $\mathrm{As}^{5+}, \mathrm{Hg}^{2+}$, and $\mathrm{Mn}^{2+}$ and other alkaline metals such as $\mathrm{Ba}^{2+}$ and $\mathrm{Sr}^{2+}$ bind to DREAM. Based on tryptophan emission and 1,8-emission study we conclude that either $\mathrm{As}^{5+}, \mathrm{Hg}^{2+}, \mathrm{Mn}^{2+}, \mathrm{Ba}^{2+}$, or $\mathrm{Sr}^{2+}$ does not bind to DREAM or binding of $\mathrm{As}^{5+}, \mathrm{Hg}^{2+}, \mathrm{Mn}^{2+}, \mathrm{Ba}^{2+}$, or $\mathrm{Sr}^{2+}$ does not trigger structural rearrangements. 
In chapter 7 , we investigated impact of Lys 87 to Ala mutation on DREAM secondary and tertiary structures and interaction with intracellular partners. Accelerated molecular dynamics data indicate the presence of a salt bridge between Lys87 and Asp 165 that couples the loop between helix-1 and helix-2 in the N-terminal domain with the EF-3 in the C- terminal domain We postulated that the salt bridge involved in the propagation of calcium-triggered structural changes between the $\mathrm{C}$ - terminal and $\mathrm{N}$ - terminal domain. To determine the contribution of Lys87 to the interdomain communication, we mutated Lys 87 to Ala in order to break the salt bridge and investigated the impact of the mutation on DREAM secondary and tertiary structures and DREAM interactions with interacting partners. Our data reveal that the emission maximum of Trp169 in DREAM(K87A) is 5 $\mathrm{nm}$ red-shifted compared with corresponding wild-type protein, suggesting Trp169 is more solvent exposed in DREAM(K87A). The increase in solvent accessibility can be explained by the fact that the absence of salt bridge destabilizes the protein structure. Also disrupting the interdomain coummunication by mutating Lys 87 to Ala prevents DREAM interaction with PS1HL9 and site-1 and site-2 peptides of the T-1 domain of Kv channel. This result is consistent with the notion of 1,8-ANS lifetime data where DREAM shows a weaker affinity for hydrophobic molecule 1,8-ANS.

In chapter 8, we mutated glutamate residue at $12^{\text {th }}$ position of EF-3 (DREAM(E186Q)) or EF-4 (DREAM(E234Q)) to glutamine for destroying $\mathrm{Ca}^{2+}$ binding properties of these EF-hands and investigated impact of mutations on $\mathrm{Ca}^{2+}$-triggered secondary and tertiary structural rearrangements of the protein and $\mathrm{Ca}^{2+}$-induced modulation of DREAM interaction with T-1 domain of $\mathrm{Kv}$ channel. On the basis of $\mathrm{CD}$, Trp emission, and 1,8ANS emission data, we conclude that $\mathrm{Ca}^{2+}$ binding in EF-4 causes secondary and tertiary 
structural rearrangement of the proteins. We also demonstrated that EF-3 or EF-4 inactivation inhibits DREAM interaction with $\mathrm{Kv} 4.3(2-22)$ peptides of T-1 domain of $\mathrm{Kv}$ channel, but EF-3 or EF-4 inactivation doesn't impact DREAM interaction with Kv4.3(7090) peptides of $\mathrm{T}-1$ domain of $\mathrm{Kv}$ channel. $\mathrm{Kv} 4.3(2-22)$ binds to $\mathrm{N}$ - and $\mathrm{C}$ - terminal hydrophobic patches on DREAM surface; inactivating either EF-3 or EF-4 might disturb big hydrophobic patches in the C-terminal of DREAM and subsequently prevents interaction between DREAM and Kv4.3(2-22). Kv4.3(70-90) binding site is located at the N-terminus of DREAM. Inactivating EF-3 or EF-4 at the C-terminus of DREAM may likely not have impact on Kv4.3(70-90) binding at the N-terminus. 


\section{LIST OF REFERENCES}

ABD RASHID, N., HAPIDIN, H., ABDULLAH, H., ISMAIL, Z. and LONG, I., 2017. Nicotine-prevented learning and memory impairment in REM sleep-deprived rat is modulated by DREAM protein in the hippocampus. Brain and behavior, 7(6), pp. e00704.

AMES, J.B. and LIM, S., 2012. Molecular structure and target recognition of neuronal calcium sensor proteins. Biochimica et Biophysica Acta (BBA)-General Subjects, 1820(8), pp. 1205-1213.

AMES, J.B., TANAKA, T., STRYER, L. and IKURA, M., 1996. Portrait of a myristoyl switch protein. Current opinion in structural biology, 6(4), pp. 432-438.

AN, W.F., BOWLBY, M.R., BETTY, M., CAO, J., LING, H., MENDOZA, G., HINSON, J.W., MATTSSON, K.I., STRASSLE, B.W. and TRIMMER, J.S., 2000. Modulation of Atype potassium channels by a family of calcium sensors. Nature, 403(6769), pp. 553-556.

ANDERSSON, A., FORSEN, S., THULIN, E. and VOGEL, J.J., 1983. Cadmium-113 nuclear magnetic resonance studies of proteolytic fragments of calmodulin: assignment of strong and weak cation binding sites. Biochemistry, 22(10), pp. 2309-2313.

ARAVIND, P., CHANDRA, K., REDDY, P.P., JEROMIN, A., CHARY, K. and SHARMA, Y., 2008. Regulatory and structural EF-hand motifs of neuronal calcium sensor-1: Mg 2 modulates $\mathrm{Ca} 2$ binding, $\mathrm{Ca} 2$-induced conformational changes, and equilibrium unfolding transitions. Journal of molecular biology, 376(4), pp. 1100-1115.

ARCHIBALD, F.S. and TYREE, C., 1987. Manganese poisoning and the attack of trivalent manganese upon catecholamines. Archives of Biochemistry and Biophysics, 256(2), pp. $638-650$.

ARGOS, M., KALRA, T., RATHOUZ, P.J., CHEN, Y., PIERCE, B., PARVEZ, F., ISLAM, T., AHMED, A., RAKIBUZ-ZAMAN, M. and HASAN, R., 2010. Arsenic exposure from drinking water, and all-cause and chronic-disease mortalities in Bangladesh (HEALS): a prospective cohort study. The Lancet, 376(9737), pp. 252-258.

AZAM, S. and MIKSOVSKA, J., 2018. Pb2 Binds to DREAM and Modulates Its Interactions with Binding Partners: a Link between Neuronal Calcium Sensors and $\mathrm{Pb} 2$ Neurotoxicity. ACS chemical neuroscience, 10 (3), 1263-1272 .

AZAM, S., ST LUIS, G. and MIKSOVSKA, J., 2019. Cadmium association to DREAM promotes DREAM interactions with intracellular partners in a similar manner as its physiological ligand, calcium. Metallomics, 10.1039/C9MT00059C . 
BÄHRE, H., DANKER, K.Y., STASCH, J., KAEVER, V. and SEIFERT, R., 2014. Nucleotidyl cyclase activity of soluble guanylyl cyclase in intact cells. Biochemical and biophysical research communications, 443(4), pp. 1195-1199.

BAHRING, R., DANNENBERG, J., PETERS, H.C., LEICHER, T., PONGS, O. and ISBRANDT, D., 2001. Conserved Kv4 N-terminal domain critical for effects of $\mathrm{Kv}$ channel-interacting protein 2.2 on channel expression and gating. The Journal of biological chemistry, 276(26), pp. 23888-23894.

BARRY, P.S. and MOSSMAN, D.B., 1970. Lead concentrations in human tissues. British journal of industrial medicine, 27(4), pp. 339-351.

BASHA, M.R., MURALI, M., SIDDIQI, H.K., GHOSAL, K., SIDDIQI, O.K., LASHUEL, H.A., GE, Y.W., LAHIRI, D.K. and ZAWIA, N.H., 2005. Lead (Pb) exposure and its effect on APP proteolysis and Abeta aggregation. FASEB journal : official publication of the Federation of American Societies for Experimental Biology, 19(14), pp. 2083-2084.

BASHA, M.R., WEI, W., BAKHEET, S.A., BENITEZ, N., SIDDIQI, H.K., GE, Y.W., LAHIRI, D.K. and ZAWIA, N.H., 2005. The fetal basis of amyloidogenesis: exposure to lead and latent overexpression of amyloid precursor protein and beta-amyloid in the aging brain. The Journal of neuroscience : the official journal of the Society for Neuroscience, 25(4), pp. 823-829.

BERRIDGE, M.J., BOOTMAN, M.D. and RODERICK, H.L., 2003. Calcium: calcium signalling: dynamics, homeostasis and remodelling. Nature reviews Molecular cell biology, 4(7), pp. 517.

BERRIDGE, M.J., LIPP, P. and BOOTMAN, M.D., 2000. The versatility and universality of calcium signalling. Nature reviews Molecular cell biology, 1(1), pp. 11.

BEZPROZVANNY, I., 2007. Inositol 1, 4, 5-tripshosphate receptor, calcium signalling and Huntington's disease. Calcium Signalling and Disease. Springer, pp. 323-335.

BEZPROZVANNY, I., 2009. Calcium signaling and neurodegenerative diseases. Trends in molecular medicine, 15(3), pp. 89-100.

BLOCK, C., FREYERMUTH, S., BEYERSMANN, D. and MALVIYA, A., 1992. Role of cadmium in activating nuclear protein kinase $\mathrm{C}$ and the enzyme binding to nuclear protein. Journal of Biological Chemistry, 267(28), pp. 19824-19828.

BOECKEL, G.R. and EHRLICH, B.E., 2018. NCS-1 is a regulator of calcium signaling in health and disease. Biochimica et Biophysica Acta (BBA)-Molecular Cell Research, 1865,11, 1660-1667. 
BOLSHAKOV, V.Y. and SIEGELBAUM, S.A., 1994. Postsynaptic induction and presynaptic expression of hippocampal long-term depression. Science (New York, N.Y.), 264(5162), pp. 1148-1152.

BOURDEAU, M., LAPLANTE, I., LAURENT, C. and LACAILLE, J., 2011. KChIP1 modulation of Kv4. 3-mediated A-type $\mathrm{K}$ currents and repetitive firing in hippocampal interneurons. Neuroscience, 176, pp. 173-187.

BRANCO, V., CANÁRIO, J., LU, J., HOLMGREN, A. and CARVALHO, C., 2012. Mercury and selenium interaction in vivo: effects on thioredoxin reductase and glutathione peroxidase. Free Radical Biology and Medicine, 52(4), pp. 781-793.

BRAUNEWELL, K. and GUNDELFINGER, E.D., 1999. Intracellular neuronal calcium sensor proteins: a family of EF-hand calcium-binding proteins in search of a function. Cell and tissue research, 295(1), pp. 1-12.

BRAUNEWELL, K. and SZANTO, A.J.K., 2009. Visinin-like proteins (VSNLs): interaction partners and emerging functions in signal transduction of a subfamily of neuronal Ca2 -sensor proteins. Cell and tissue research, 335(2), pp. 301-316.

BRINI, M., CALÌ, T., OTTOLINI, D. and CARAFOLI, E., 2014. Neuronal calcium signaling: function and dysfunction. Cellular and molecular life sciences, 71(15), pp. 2787-2814.

BROPHY, M.B. and NOLAN, E.M., 2015. Manganese and microbial pathogenesis: sequestration by the mammalian immune system and utilization by microorganisms. $A C S$ chemical biology, 10(3), pp. 641-651.

BROWN, K.M. and TRACY, D.K., 2013. Lithium: the pharmacodynamic actions of the amazing ion. Therapeutic advances in psychopharmacology, 3(3), pp. 163-176.

BURGOYNE, R.D., 2007. Neuronal calcium sensor proteins: generating diversity in neuronal Ca2 signalling. Nature Reviews Neuroscience, 8(3), pp. 182-193.

BURGOYNE, R.D. and WEISS, J.L., 2001. The neuronal calcium sensor family of Ca2+binding proteins. The Biochemical journal, 353(Pt 1), pp. 1-12.

BUXBAUM, J.D., CHOI, E., LUO, Y., LILLIEHOOK, C., CROWLEY, A.C., MERRIAM, D.E. and WASCO, W., 1998. Calsenilin: a calcium-binding protein that interacts with the presenilins and regulates the levels of a presenilin fragment. Nature medicine, 4(10), pp. 1177-1181.

CADE, J.F., 1949. Lithium salts in the treatment of psychotic excitement. Medical journal of Australia, 2(10), pp. 349-352. 
CALLSEN, B., ISBRANDT, D., SAUTER, K., HARTMANN, L.S., PONGS, O. and BÄHRING, R., 2005. Contribution of N-and C-terminal channel domains to Kv channel interacting proteins in a mammalian cell line. The Journal of physiology, 568(2), pp. 397412.

CALVERT, P.D., KLENCHIN, V.A. and BOWNDS, M.D., 1995. Rhodopsin kinase inhibition by recoverin. Function of recoverin myristoylation. The Journal of biological chemistry, 270(41), pp. 24127-24129.

CANFIELD, R.L., HENDERSON JR, C.R., CORY-SLECHTA, D.A., COX, C., JUSKO, T.A. and LANPHEAR, B.P., 2003. Intellectual impairment in children with blood lead concentrations below $10 \mu \mathrm{g}$ per deciliter. New England journal of medicine, 348(16), pp. 1517-1526.

CAO, Y., CHEN, A., RADCLIFFE, J., DIETRICH, K.N., JONES, R.L., CALDWELL, K. and ROGAN, W.J., 2009. Postnatal cadmium exposure, neurodevelopment, and blood pressure in children at 2, 5, and 7 years of age. Environmental health perspectives, 117(10), pp. 1580-1586.

CAPEL, I.D., PINNOCK, M.H., DORRELL, H.M., WILLIAMS, D.C. and GRANT, E.C., 1981. Comparison of concentrations of some trace, bulk, and toxic metals in the hair of normal and dyslexic children. Clinical chemistry, 27(6), pp. 879-881.

CARAFOLI, E., SANTELlA, L., BRANCA, D. and BRINI, M., 2001. Generation, control, and processing of cellular calcium signals. Critical reviews in biochemistry and molecular biology, 36(2), pp. 107-260.

CARDAMONE, M. and PURI, N.K., 1992. Spectrofluorimetric assessment of the surface hydrophobicity of proteins. The Biochemical journal, 282 (Pt 2), pp. 589-593.

CAROCCI, A., ROVITO, N., SINICROPI, M.S. and GENCHI, G., 2014. Mercury toxicity and neurodegenerative effects. Reviews of environmental contamination and toxicology. Springer, pp. 1-18.

CARRION, A.M., LINK, W.A., LEDO, F., MELLSTRÖM, B. and NARANJO, J.R., 1999. DREAM is a Ca2\&plus;-regulated transcriptional repressor. Nature, 398(6722), pp. 80-84.

CARRION, A.M., MELLSTROM, B. and NARANJO, J.R., 1998. Protein kinase Adependent derepression of the human prodynorphin gene via differential binding to an intragenic silencer element. Molecular and cellular biology, 18(12), pp. 6921-6929.

CARVALHO, C.M., CHEW, E.H., HASHEMY, S.I., LU, J. and HOLMGREN, A., 2008. Inhibition of the human thioredoxin system. A molecular mechanism of mercury toxicity. The Journal of biological chemistry, 283(18), pp. 11913-11923. 
CHANG, K.N., LEE, T.C., TAM, M.F., CHEN, Y.C., LEE, L.W., LEE, S.Y., LIN, P.J. and HUANG, R.N., 2003. Identification of galectin I and thioredoxin peroxidase II as two arsenic-binding proteins in Chinese hamster ovary cells. The Biochemical journal, 371( $\mathrm{Pt}$ 2), pp. 495-503.

CHAO, S.H., BU, C.H. and CHEUNG, W.Y., 1995. Stimulation of myosin light-chain kinase by $\mathrm{Cd} 2$ and $\mathrm{Pb} 2$. Archives of Toxicology, 69(3), pp. 197-203.

CHAO, S.L., MOSS, J.M. and HARRY, G.J., 2007. Lead-induced alterations of apoptosis and neurotrophic factor mRNA in the developing rat cortex, hippocampus, and cerebellum. Journal of Biochemical and Molecular Toxicology, 21(5), pp. 265-272.

CHEN, C., WANG, S., CHIOU, J., TSENG, C., CHIOU, H., HSUEH, Y., CHEN, S., WU, M. and LAI, M., 2007. Arsenic and diabetes and hypertension in human populations: a review. Toxicology and applied pharmacology, 222(3), pp. 298-304.

CHEN, Y., GRAZIANO, J.H., PARVEZ, F., LIU, M., SLAVKOVICH, V., KALRA, T., ARGOS, M., ISLAM, T., AHMED, A., RAKIBUZ-ZAMAN, M., HASAN, R., SARWAR, G., LEVY, D., VAN GEEN, A. and AHSAN, H., 2011. Arsenic exposure from drinking water and mortality from cardiovascular disease in Bangladesh: prospective cohort study. BMJ (Clinical research ed.), 342, pp. d2431.

CHENG, H.M., PITCHER, G.M., LAVIOLETTE, S.R., WHISHAW, I.Q., TONG, K.I., KOCKERITZ, L.K., WADA, T., JOZA, N.A., CRACKOWER, M. and GONCALVES, J., 2002. DREAM is a critical transcriptional repressor for pain modulation. Cell, 108(1), pp. $31-43$.

CHOONG, G., LIU, Y. and TEMPLETON, D.M., 2014. Interplay of calcium and cadmium in mediating cadmium toxicity. Chemico-biological interactions, 211, pp. 54-65.

CHOUCHANE, S. and SNOW, E.T., 2001. In vitro effect of arsenical compounds on glutathione-related enzymes. Chemical research in toxicology, 14(5), pp. 517-522.

CHRISTIE, B.R., MAGEE, J.C. and JOHNSTON, D., 1996. Dendritic calcium channels and hippocampal long-term depression. Hippocampus, 6(1), pp. 17-23.

CLAPHAM, D.E., 2007. Calcium signaling. Cell, 131(6), pp. 1047-1058.

CLARKSON, T.W. and MAGOS, L., 2006. The toxicology of mercury and its chemical compounds. Critical reviews in toxicology, 36(8), pp. 609-662.

CLARKSON, T.W., 2002. The three modern faces of mercury. Environmental health perspectives, 110 Suppl 1, pp. 11-23. 
COLLINS, J.H., 1974. Homology of myosin light chains, troponin-C and parvalbumins deduced from comparison of their amino acid sequences. Biochemical and biophysical research communications, 58(1), pp. 301-308.

COLLINS, J.H., POTTER, J.D., HORN, M.J., WILSHIRE, G. and JACKMAN, N., 1973. The amino acid sequence of rabbit skeletal muscle troponin $\mathrm{C}$ : Gene replication and homology with calcium-binding proteins from carp and hake muscle. FEBS letters, 36(3), pp. 268-272.

COSTIGAN, M. and WOOLF, C.J., 2002. No DREAM, no pain: closing the spinal gate. Cell, 108(3), pp. 297-300.

CRABTREE, M.D. and SHAMMAS, S.L., 2018. Stopped-Flow Kinetic Techniques for Studying Binding Reactions of Intrinsically Disordered Proteins. Methods in enzymology, 611, pp. 423-457.

DAMO, S.M., KEHL-FIE, T.E., SUGITANI, N., HOLT, M.E., RATHI, S., MURPHY, W.J., ZHANG, Y., BETZ, C., HENCH, L., FRITZ, G., SKAAR, E.P. and CHAZIN, W.J., 2013. Molecular basis for manganese sequestration by calprotectin and roles in the innate immune response to invading bacterial pathogens. Proceedings of the National Academy of Sciences of the United States of America, 110(10), pp. 3841-3846.

DE FERRARI, G., CHACON, M., BARRIA, M., GARRIDO, J., GODOY, J., OLIVARES, G., REYES, A., ALVAREZ, A., BRONFMAN, M. and INESTROSA, N., 2003. Activation of Wnt signaling rescues neurodegeneration and behavioral impairments induced by $\beta$-amyloid fibrils. Molecular psychiatry, 8(2), pp. 195.

DEIBLER, K. and BASU, P., 2013. Continuing issues with lead: recent advances in detection. European journal of inorganic chemistry, 2013(7), pp. 1086-1096.

DIZHOOR, A.M., LOWE, D.G., OLSHEVSKAYA, E.V., LAURA, R.P. and HURLEY, J.B., 1994. The human photoreceptor membrane guanylyl cyclase, RetGC, is present in outer segments and is regulated by calcium and a soluble activator. Neuron, 12(6), pp. $1345-1352$.

DIZHOOR, A.M., RAY, S., KUMAR, S., NIEMI, G., SPENCER, M., BROLLEY, D., WALSH, K.A., PHILIPOV, P.P., HURLEY, J.B. and STRYER, L., 1991. Recoverin: a calcium sensitive activator of retinal rod guanylate cyclase. Science (New York, N.Y.), 251(4996), pp. 915-918.

D'ONOFRIO, S., HYDE, J. and GARCIA-RILL, E., 2017. Interaction between neuronal calcium sensor protein 1 and lithium in pedunculopontine neurons. Physiological reports, 5(7), 
D'ONOFRIO, S., KEZUNOVIC, N., HYDE, J.R., LUSTER, B., MESSIAS, E., URBANO, F.J. and GARCIA-RILL, E., 2015. Modulation of gamma oscillations in the pedunculopontine nucleus by neuronal calcium sensor protein-1: relevance to schizophrenia and bipolar disorder. Journal of neurophysiology, 113(3), pp. 709-719.

DOYLE, D., 2009. Notoriety to respectability: a short history of arsenic prior to its present day use in haematology. British journal of haematology, 145(3), pp. 309-317.

DUDEV, T., MAZMANIAN, K. and LIM, C., 2018. Competition between Li and $\mathrm{Na}$ in sodium transporters and receptors: Which $\mathrm{Na}$-Binding sites are "therapeutic" Li targets? Chemical Science, 9(17), pp. 4093-4103.

EDINGTON, S.C., GONZALEZ, A., MIDDENDORF, T.R., HALLING, D.B., ALDRICH, R.W. and BAIZ, C.R., 2018. Coordination to lanthanide ions distorts binding site conformation in calmodulin. Proceedings of the National Academy of Sciences of the United States of America, 115(14), pp. E3126-E3134.

ELLIS, P.D., STRANG, P. and POTTER, J.D., 1984. Cadmium-substituted skeletal troponin C. Cadmium-113 NMR spectroscopy and metal binding investigations. The Journal of biological chemistry, 259(16), pp. 10348-10356.

EL-MARAGHY, S.A., GAD, M.Z., FAHIM, A.T. and HAMDY, M.A., 2001. Effect of cadmium and aluminum intake on the antioxidant status and lipid peroxidation in rat tissues. Journal of Biochemical and Molecular Toxicology, 15(4), pp. 207-214.

ENGEL, T., GOÑI-OLIVER, P., LUCAS, J.J., AVILA, J. and HERNÁNDEZ, F., 2006. Chronic lithium administration to FTDP-17 tau and GSK-3 $\beta$ overexpressing mice prevents tau hyperphosphorylation and neurofibrillary tangle formation, but pre-formed neurofibrillary tangles do not revert. Journal of neurochemistry, 99(6), pp. 1445-1455.

ESSEN, L., PERISIC, O., CHEUNG, R., KATAN, M. and WILLIAMS, R.L., 1996. Crystal structure of a mammalian phosphoinositide-specific phospholipase C $\delta$. Nature, 380(6575), pp. 595.

FLAHERTY, K.M., ZOZULYA, S., STRYER, L. and MCKAY, D.B., 1993. Threedimensional structure of recoverin, a calcium sensor in vision. Cell, 75(4), pp. 709-716.

FONTÁN-LOZANO, Á., ROMERO-GRANADOS, R., DEL-POZO-MARTÍN, Y., SUÁREZ-PEREIRA, I., DELGADO-GARCÍA, J.M., PENNINGER, J.M. and CARRIÓN, Á.M., 2009. Lack of DREAM protein enhances learning and memory and slows brain aging. Current Biology, 19(1), pp. 54-60.

FORLENZA, O.V., DE-PAULA, VANESSA DE JESUS RODRIGUES and DINIZ, B., 2014. Neuroprotective effects of lithium: implications for the treatment of Alzheimer's 
disease and related neurodegenerative disorders. ACS chemical neuroscience, 5(6), pp. $443-450$.

FORSÉN, S., THULIN, E., DRAKENBERG, T., KREBS, J. and SEAMON, K., 1980. A $113 C d$ NMR study of calmodulin and its interaction with calcium, magnesium and trifluoperazine. FEBS letters, 117(1-2), pp. 189-194.

FRIEDMAN, R., 2014. Structural and computational insights into the versatility of cadmium binding to proteins. Dalton Transactions, 43(7), pp. 2878-2887.

FULCHER, L.M., 2015. Thermodynamic and spectroscopic studies of $\mathrm{Cd}^{2+}$ binding to the regulatory domain and full length human cardiac troponin $\mathrm{C}(\mathrm{HcTnC})$ : elucidating plausible $\mathrm{Cd}^{2+}$ binding sites.

GABBIANI, G., BAIC, D. and DEZIEL, C., 1967. Toxicity of cadmium for the central nervous system. Experimental neurology, 18(2), pp. 154-160.

GEORGE, S.E., SU, Z., FAN, D. and MEANS, A.R., 1993. Calmodulin-cardiac troponin $\mathrm{C}$ chimeras. Effects of domain exchange on calcium binding and enzyme activation. The Journal of biological chemistry, 268(33), pp. 25213-25220.

GERKE, V. and MOSS, S.E., 2002. Annexins: from structure to function. Physiological Reviews, 82(2), pp. 331-371.

GIFFORD, J.L., WALSH, M.P. and VOGEL, H.J., 2007. Structures and metal-ion-binding properties of the Ca2+-binding helix-loop-helix EF-hand motifs. The Biochemical journal, 405(2), pp. 199-221.

GILSTON, B.A., SKAAR, E.P. and CHAZIN, W.J., 2016. Binding of transition metals to S100 proteins. Science China Life Sciences, 59(8), pp. 792-801.

GOHARA, D.W. and DI CERA, E., 2016. Molecular Mechanisms of Enzyme Activation by Monovalent Cations. The Journal of biological chemistry, 291(40), pp. 20840-20848.

GOLDSTEIN, G.W. and AR, D., 1983. Lead activates calmodulin sensitive processes. Life Sciences, 33(10), pp. 1001-1006.

GONZALEZ, W.G., ARANGO, A.S. and MIKSOVSKA, J., 2015. Amphiphilic Residues 29-44 of DREAM N-Termini Mediate Calmodulin: DREAM Complex Formation. Biochemistry, 54(28), pp. 4391-4403.

GONZALEZ, W.G. and MIKSOVSKA, J., 2014. Application of ANS fluorescent probes to identify hydrophobic sites on the surface of DREAM. Biochimica Et Biophysica Acta (BBA)-Proteins and Proteomics, 1844(9), pp. 1472-1480. 
GONZALEZ, W.G., PHAM, K. and MIKSOVSKA, J., 2014. Modulation of the voltagegated potassium channel (Kv4.3) and the auxiliary protein (KChIP3) interactions by the current activator NS5806. The Journal of biological chemistry, 289(46), pp. 32201-32213.

GONZALEZ, W.G., RAMOS, V., DIAZ, M., GARABEDIAN, A., MOLANOAREVALO, J.C., FERNANDEZ-LIMA, F. and MIKSOVSKA, J., 2016. Characterization of the Photophysical, Thermodynamic, and Structural Properties of the Terbium(III)DREAM Complex. Biochemistry, 55(12), pp. 1873-1886.

GORKHALI, R., HUANG, K., KIRBERGER, M. and YANG, J.J., 2016. Defining potential roles of $\mathrm{Pb} 2$ in neurotoxicity from a calciomics approach. Metallomics, 8(6), pp. 563-578.

GRABAREK, Z., 2006. Structural basis for diversity of the EF-hand calcium-binding proteins. Journal of Molecular Biology, 359(3), pp. 509-525.

GRANDJEAN, P., 1978. Regional distribution of lead in human brains. Toxicology letters, 2(1), pp. 65-69.

GROVER, L.M. and TEYLER, T.J., 1990. Two components of long-term potentiation induced by different patterns of afferent activation. Nature, 347(6292), pp. 477.

GU, C. and COOPER, D.M., 2000. $\mathrm{Ca}(2+), \operatorname{Sr}(2+)$, and $\mathrm{Ba}(2+)$ identify distinct regulatory sites on adenylyl cyclase (AC) types VI and VIII and consolidate the apposition of capacitative cation entry channels and $\mathrm{Ca}(2+)$-sensitive ACs. The Journal of biological chemistry, 275(10), pp. 6980-6986.

GUILARTE, T.R., 2010. APLP1, Alzheimer's-like pathology and neurodegeneration in the frontal cortex of manganese-exposed non-human primates. Neurotoxicology, 31(5), pp. $572-574$.

GUNTER, T.E., GAVIN, C.E., ASCHNER, M. and GUNTER, K.K., 2006. Speciation of manganese in cells and mitochondria: a search for the proximal cause of manganese neurotoxicity. Neurotoxicology, 27(5), pp. 765-776.

HABERMANN, E., CROWELL, K. and JANICKI, P., 1983. Lead and other metals can substitute for $\mathrm{Ca} 2$ in calmodulin. Archives of Toxicology, 54(1), pp. 61-70.

HAIMOVICH, A., ELIAV, U. and GOLDBOURT, A., 2012. Determination of the lithium binding site in inositol monophosphatase, the putative target for lithium therapy, by magicangle-spinning solid-state NMR. Journal of the American Chemical Society, 134(12), pp. 5647-5651. 
HALLCHER, L.M. and SHERMAN, W.R., 1980. The effects of lithium ion and other agents on the activity of myo-inositol-1-phosphatase from bovine brain. The Journal of biological chemistry, 255(22), pp. 10896-10901.

HANDLEY, M.T., LIAN, L., HAYNES, L.P. and BURGOYNE, R.D., 2010. Structural and functional deficits in a neuronal calcium sensor-1 mutant identified in a case of autistic spectrum disorder. PloS one, 5(5), pp. e10534.

HARTWIG, A., BLESSING, H., SCHWERDTLE, T. and WALTER, I., 2003. Modulation of DNA repair processes by arsenic and selenium compounds. Toxicology, 193(1-2), pp. 161-169.

HARWOOD, A., 2005. Lithium and bipolar mood disorder: the inositol-depletion hypothesis revisited. Molecular psychiatry, 10(1), pp. 117.

HAYNES, L.P., SHERWOOD, M.W., DOLMAN, N.J. and BURGOYNE, R.D., 2007. Specificity, Promiscuity and Localization of ARF Protein Interactions with NCS-1 and Phosphatidylinositol-4 Kinase-III $\beta$. Traffic, 8(8), pp. 1080-1092.

HENZL, M.T., LARSON, J.D. and AGAH, S., 2004a. Influence of monovalent cation identity on parvalbumin divalent ion-binding properties. Biochemistry, $\mathbf{4 3}(10)$, pp. 27472763.

HENZL, M.T., LARSON, J.D. and AGAH, S., 2004b. Influence of monovalent cation identity on parvalbumin divalent ion-binding properties. Biochemistry, 43(10), pp. 27472763.

HOLMQVIST, M.H., CAO, J., HERNANDEZ-PINEDA, R., JACOBSON, M.D., CARROLL, K.I., SUNG, M.A., BETTY, M., GE, P., GILBRIDE, K.J., BROWN, M.E., JURMAN, M.E., LAWSON, D., SILOS-SANTIAGO, I., XIE, Y., COVARRUBIAS, M., RHODES, K.J., DISTEFANO, P.S. and AN, W.F., 2002. Elimination of fast inactivation in Kv4 A-type potassium channels by an auxiliary subunit domain. Proceedings of the National Academy of Sciences of the United States of America, 99(2), pp. 1035-1040.

HUANG, H., BIHAQI, S.W., CUI, L. and ZAWIA, N.H., 2011. In vitro Pb exposure disturbs the balance between $A \beta$ production and elimination: the role of $A \beta P P$ and neprilysin. Neurotoxicology, 32(3), pp. 300-306.

HUGHES, M.F., 2002. Arsenic toxicity and potential mechanisms of action. Toxicology letters, 133(1), pp. 1-16.

HUGHES, R.E., BRZOVIC, P.S., KLEVIT, R.E. and HURLEY, J.B., 1995. Calciumdependent solvation of the myristoyl group of recoverin. Biochemistry, 34(36), pp. 1141011416. 
HUMPHREY, W., DALKE, A. and SCHULTEN, K., 1996. VMD: visual molecular dynamics. Journal of Molecular Graphics, 14(1), pp. 33-38.

IARC WORKING GROUP ON THE EVALUATION OF THE CARCINOGENIC RISK OF CHEMICALS TO HUMANS, 1993. Beryllium, cadmium, mercury, and exposures in the glass manufacturing industry. International Agency for Research on Cancer.

IMPEY, S., MARK, M., VILLACRES, E.C., POSER, S., CHAVKIN, C. and STORM, D.R., 1996. Induction of CRE-mediated gene expression by stimuli that generate longlasting LTP in area CA1 of the hippocampus. Neuron, 16(5), pp. 973-982.

IPCS, W., 1992. Enviropnmental Health Criteria 134. Cadmium, 52-130. World Health Organization, Geneva, .

JAIN, C. and ALI, I., 2000. Arsenic: occurrence, toxicity and speciation techniques. Water research, 34(17), pp. 4304-4312.

JALILEHVAND, F., SPÅNGBERG, D., LINDQVIST-REIS, P., HERMANSSON, K., PERSSON, I. and SANDSTRÖM, M., 2001. Hydration of the calcium ion. An EXAFS, large-angle X-ray scattering, and molecular dynamics simulation study. Journal of the American Chemical Society, 123(3), pp. 431-441.

JAN, A.T., ALI, A. and HAQ, Q., 2011. Glutathione as an antioxidant in inorganic mercury induced nephrotoxicity. Journal of postgraduate medicine, 57(1), pp. 72-77.

JÄRUP, L. and ÅKESSON, A., 2009. Current status of cadmium as an environmental health problem. Toxicology and applied pharmacology, 238(3), pp. 201-208.

JEANNE, M., LALLEMAND-BREITENBACH, V., FERHI, O., KOKEN, M., LE BRAS, M., DUFFORT, S., PERES, L., BERTHIER, C., SOILIHI, H. and RAUGHT, B., 2010. PML/RARA oxidation and arsenic binding initiate the antileukemia response of As2O3. Cancer cell, 18(1), pp. 88-98.

JESU JAYA SUDAN, R. and SUDANDIRADOSS, C., 2012. Pattern prediction and coordination geometry analysis from cadmium-binding proteins: a computational approach. Acta Crystallographica Section D, 68(10), pp. 1346-1358.

JIN, T., LU, J. and NORDBERG, M., 1998. Toxicokinetics and biochemistry of cadmium with special emphasis on the role of metallothionein. Neurotoxicology, 19(4-5), pp. 529535.

JO, D., JANG, J., KIM, B., LUNDKVIST, J. and JUNG, Y., 2005. Overexpression of calsenilin enhances $\gamma$-secretase activity. Neuroscience letters, 378(1), pp. 59-64. 
JO, S., KIM, T., IYER, V.G. and IM, W., 2008. CHARMM-GUI: a web-based graphical user interface for CHARMM. Journal of computational chemistry, 29(11), pp. 1859-1865.

JOHNSON, S., 2001. Gradual micronutrient accumulation and depletion in Alzheimer's disease. Medical hypotheses, 56(6), pp. 595-597.

JOSEPH, P., MUCHNOK, T.K., KLISHIS, M.L., ROBERTS, J.R., ANTONINI, J.M., WHONG, W. and ONG, T., 2001. Cadmium-induced cell transformation and tumorigenesis are associated with transcriptional activation of c-fos, c-jun, and c-myc proto-oncogenes: role of cellular calcium and reactive oxygen species. Toxicological sciences, 61(2), pp. 295-303.

KANTER, M., UNSAL, C., AKTAS, C. and ERBOGA, M., 2016. Neuroprotective effect of quercetin against oxidative damage and neuronal apoptosis caused by cadmium in hippocampus. Toxicology and industrial health, 32(3), pp. 541-550.

KAWAMOTO, E.M., VIVAR, C. and CAMANDOLA, S., 2012. Physiology and pathology of calcium signaling in the brain. Frontiers in pharmacology, 3, pp. 61.

KERN, M., WISNIEWSKI, M., CABELL, L. and AUDESIRK, G., 2000. Inorganic lead and calcium interact positively in activation of calmodulin. Neurotoxicology, 21(3), pp. 353-363.

KHROMOVA, V. and MYSHKIN, A., 2000. Coagulation of bovine methemoglobin mercurated at reactive sulfur in the presence of ligands with a high affinity for Hg (II). Russian Journal of General Chemistry, 70(4), pp. 635-639.

KIRBERGER, M., WONG, H.C., JIANG, J. and YANG, J.J., 2013. Metal toxicity and opportunistic binding of $\mathrm{Pb} 2$ in proteins. Journal of inorganic biochemistry, 125, pp. 4049.

KIRBERGER, M. and YANG, J.J., 2008. Structural differences between Pb 2 -and Ca 2 binding sites in proteins: implications with respect to toxicity. Journal of inorganic biochemistry, 102(10), pp. 1901-1909.

KITCHIN, K.T. and WALLACE, K., 2005. Arsenite binding to synthetic peptides based on the $\mathrm{Zn}$ finger region and the estrogen binding region of the human estrogen receptor- $\alpha$. Toxicology and applied pharmacology, 206(1), pp. 66-72.

KOH, P.O., UNDIE, A.S., KABBANI, N., LEVENSON, R., GOLDMAN-RAKIC, P.S. and LIDOW, M.S., 2003. Up-regulation of neuronal calcium sensor-1 (NCS-1) in the prefrontal cortex of schizophrenic and bipolar patients. Proceedings of the National Academy of Sciences of the United States of America, 100(1), pp. 313-317. 
KONYAEVA, V. and MYSHKIN, A., 1998a. Effect of weak chemical actions on coagulation of oxyhemoglobin mercurated at reactive sulfur. III. Effects of EDTA and Thiourea. Russian journal of general chemistry, 68(4), pp. 643-649.

KONYAEVA, V. and MYSHKIN, A., 1998b. Effect of weak chemical actions on coagulation of oxyhemoglobin mercurated at reactive sulfur. III. Effects of EDTA and Thiourea. Russian journal of general chemistry, 68(4), pp. 643-649.

KONYAEVA, V. and MYSHKIN, A., 1998c. Effect of weak chemical interactions on coagulation of oxyhemoglobin mercurated at reactive sulfur. I. Influence of Tris (hydroxymethyl) aminomethane. Russian journal of general chemistry, 68(4), pp. 630-636.

KRETSINGER, R.H. and NOCKOLDS, C.E., 1973. Carp muscle calcium-binding protein. II. Structure determination and general description. The Journal of biological chemistry, 248(9), pp. 3313-3326.

KUMAR, R., AGARWAL, A.K. and SETH, P.K., 1996. Oxidative stress-mediated neurotoxicity of cadmium. Toxicology letters, 89(1), pp. 65-69.

KUMAR, S., AHMAD, E., KUMAR, S., KHAN, R.H. and GOURINATH, S., 2012. Flexibility of EF-hand motifs: structural and thermodynamic studies of Calcium Binding Protein-1 from Entamoeba histolytica with $\mathrm{Pb} 2, \mathrm{Ba} 2$, and $\mathrm{Sr}$ 2. BMC biophysics, 5(1), pp. 15.

KUNJILWAR, K., QIAN, Y. and PFAFFINGER, P.J., 2013. Functional stoichiometry underlying $\mathrm{KC}$ h IP regulation of Kv4. 2 functional expression. Journal of neurochemistry, 126(4), pp. 462-472.

KURSULA, P. and MAJAVA, V., 2007. A structural insight into lead neurotoxicity and calmodulin activation by heavy metals. Acta Crystallographica Section F: Structural Biology and Crystallization Communications, 63(8), pp. 653-656.

LAFUENTE, A., FENÁNDEZ-REY, E., SEARA, R., PÉREZ-LORENZO, M. and ESQUIFINO, A., 2001. Alternate cadmium exposure differentially affects amino acid metabolism within the hypothalamus, median eminence, striatum and prefrontal cortex of male rats. Neurochemistry international, 39(3), pp. 187-192.

LAFUENTE, A., GONZALEZ-CARRACEDO, A., ROMERO, A. and ESQUIFINO, A., 2003. Effect of cadmium on 24-h variations in hypothalamic dopamine and serotonin metabolism in adult male rats. Experimental brain research, 149(2), pp. 200-206.

LAKHIN, A., EFREMOVA, A., MAKAROVA, I., GRISHINA, E., SHRAM, S., TARANTUL, V. and GENING, L., 2013. Effect of Mn (II) on error-prone DNA polymerase iota activity in extracts from human normal and tumor cells. Molecular Genetics, Microbiology and Virology, 28(1), pp. 1-7. 
LAKHIN, A., TARANTUL, V. and GENING, L., 2014. Manganese-induced infidelity of DNA synthesis as a possible cause of manganism. Molecular Genetics, Microbiology and Virology, 29(1), pp. 16-22.

LEDO, F., KREMER, L., MELLSTROM, B. and NARANJO, J.R., 2002. Ca2+-dependent block of CREB-CBP transcription by repressor DREAM. The EMBO journal, 21(17), pp. 4583-4592.

LEROY, K., ANDO, K., HÉRAUD, C., YILMAZ, Z., AUTHELET, M., BOEYNAEMS, J., BUÉE, L., DE DECKER, R. and BRION, J., 2010. Lithium treatment arrests the development of neurofibrillary tangles in mutant tau transgenic mice with advanced neurofibrillary pathology. Journal of Alzheimer's Disease, 19(2), pp. 705-719.

LI, X., LV, Y., YU, S., ZHAO, H. and YAO, L., 2012. The effect of cadmium on A $\beta$ levels in APP/PS1 transgenic mice. Experimental and therapeutic medicine, 4(1), pp. 125-130.

LIANG, P., WANG, H., CHEN, H., CUI, Y., GU, L., CHAI, J. and WANG, K., 2009. Structural Insights into KChIP4a Modulation of Kv4.3 Inactivation. The Journal of biological chemistry, 284(8), pp. 4960-4967.

LIM, S., DIZHOOR, A. and AMES, J., 2014. Structural diversity of neuronal calcium sensor proteins and insights for activation of retinal guanylyl cyclase by GCAP1. Frontiers in molecular neuroscience, 7, pp. 19.

LIM, S., PESHENKO, I., DIZHOOR, A. and AMES, J.B., 2009. Effects of Ca2 , Mg2 , and myristoylation on guanylyl cyclase activating protein 1 structure and stability. Biochemistry, 48(5), pp. 850-862.

LIM, D., FEDRIZZI, L., TARTARI, M., ZUCCATO, C., CATTANEO, E., BRINI, M. and CARAFOLI, E., 2008. Calcium homeostasis and mitochondrial dysfunction in striatal neurons of Huntington disease. The Journal of biological chemistry, 283(9), pp. 57805789 .

LIN, C., HUANG, C., CHEN, W., CHANG, Y., DING, W., LIN, M., WU, S. and HUANG, R., 2006. Characterization of the interaction of galectin-1 with sodium arsenite. Chemical research in toxicology, 19(3), pp. 469-474.

LISS, B., FRANZ, O., SEWING, S., BRUNS, R., NEUHOFF, H. and ROEPER, J., 2001. Tuning pacemaker frequency of individual dopaminergic neurons by Kv4.3L and KChip3.1 transcription. The EMBO journal, 20(20), pp. 5715-5724.

LONG, G.J., ROSEN, J.F. and SCHANNE, F.A., 1994. Lead activation of protein kinase C from rat brain. Determination of free calcium, lead, and zinc by 19F NMR. The Journal of biological chemistry, 269(2), pp. 834-837. 
LOPEZ, E., ARCE, C., OSET-GASQUE, M., CANADAS, S. and GONZALEZ, M., 2006. Cadmium induces reactive oxygen species generation and lipid peroxidation in cortical neurons in culture. Free Radical Biology and Medicine, 40(6), pp. 940-951.

LOPEZ, E., FIGUEROA, S., OSET-GASQUE, M. and GONZALEZ, M., 2003. Apoptosis and necrosis: two distinct events induced by cadmium in cortical neurons in culture. British journal of pharmacology, 138(5), pp. 901-911.

LOUIS-JEUNE, C., ANDRADE-NAVARRO, M.A. and PEREZ-IRATXETA, C., 2012. Prediction of protein secondary structure from circular dichroism using theoretically derived spectra. Proteins: Structure, Function, and Bioinformatics, 80(2), pp. 374-381.

LU, J., CHEW, E.H. and HOLMGREN, A., 2007. Targeting thioredoxin reductase is a basis for cancer therapy by arsenic trioxide. Proceedings of the National Academy of Sciences of the United States of America, 104(30), pp. 12288-12293.

LUCCHINI, R., PLACIDI, D., CAGNA, G., FEDRIGHI, C., OPPINI, M., PELI, M. and ZONI, S., 2017. Manganese and developmental neurotoxicity. Neurotoxicity of Metals. Springer, pp. 13-34.

LUGO, J.N., BREWSTER, A.L., SPENCER, C.M. and ANDERSON, A.E., 2012. Kv4.2 knockout mice have hippocampal-dependent learning and memory deficits. Learning \& memory (Cold Spring Harbor, N.Y.), 19(5), pp. 182-189.

LUSIN, J.D., VANAROTTI, M., LI, C., VALIVETI, A. and AMES, J.B., 2008. NMR structure of DREAM: Implications for $\mathrm{Ca} 2$-dependent DNA binding and protein dimerization. Biochemistry, 47(8), pp. 2252-2264.

MAKINO, C.L., DODD, R.L., CHEN, J., BURNS, M.E., ROCA, A., SIMON, M.I. and BAYLOR, D.A., 2004. Recoverin regulates light-dependent phosphodiesterase activity in retinal rods. The Journal of general physiology, 123(6), pp. 729-741.

MANCA, D., RICARD, A.C., TROTTIER, B. and CHEVALIER, G., 1991. Studies on lipid peroxidation in rat tissues following administration of low and moderate doses of cadmium chloride. Toxicology, 67(3), pp. 303-323.

MARCUS, Y., 1991. Thermodynamics of solvation of ions. Part 5.-Gibbs free energy of hydration at 298.15 K. Journal of the Chemical Society, Faraday Transactions, 87(18), pp. 2995-2999.

MARKOVAC, J. and GOLDSTEIN, G.W., 1988. Picomolar concentrations of lead stimulate brain protein kinase C. Nature, 334(6177), pp. 71-73. 
MARLOWE, M., ERRERA, J. and JACOBS, J., 1983. Increased lead and cadmium burdens among mentally retarded children and children with borderline intelligence. American Journal of Mental Deficiency, 87(5), 477-483.

MARMOL, F., 2008. Lithium: bipolar disorder and neurodegenerative diseases Possible cellular mechanisms of the therapeutic effects of lithium. Progress in NeuroPsychopharmacology and Biological Psychiatry, 32(8), pp. 1761-1771.

MARTINEZ-FINLEY, E.J., GAVIN, C.E., ASCHNER, M. and GUNTER, T.E., 2013. Manganese neurotoxicity and the role of reactive oxygen species. Free radical biology and medicine, 62, pp. 65-75.

MASON, L.H., HARP, J.P. and HAN, D.Y., 2014. Pb neurotoxicity: neuropsychological effects of lead toxicity. BioMed research international, 2014, pp. 840547.

MATTSON, M.P., 2007. Calcium and neurodegeneration. Aging cell, 6(3), pp. 337-350.

MATTSON, M.P., 2004. Pathways towards and away from Alzheimer's disease. Nature, 430(7000), pp. 631.

MAZZEI, G.J., GIRARD, P.R. and KUO, J., 1984. Environmental pollutant Cd2 biphasically and differentially regulates myosin light chain kinase and phospholipid/Ca2 dependent protein kinase. FEBS letters, 173(1), pp. 124-128.

MCCUE, H.V., HAYNES, L.P. and BURGOYNE, R.D., 2010. The diversity of calcium sensor proteins in the regulation of neuronal function. Cold Spring Harbor perspectives in biology, 2(8), pp. a004085.

MCNEIL, A.K., RESCHER, U., GERKE, V. and MCNEIL, P.L., 2006. Requirement for annexin A1 in plasma membrane repair. The Journal of biological chemistry, 281(46), pp. 35202-35207.

MÉNDEZ-ARMENTA, M. and RIOS, C., 2007. Cadmium neurotoxicity. Environmental toxicology and pharmacology, 23(3), pp. 350-358.

MÉNDEZ-ARMENTA, M., VILLEDA-HERNÁNDEZ, J., BARROSO-MOGUEL, R., NAVA-RUÍZ, C., JIMÉNEZ-CAPDEVILLE, M.E. and RÍOS, C., 2003. Brain regional lipid peroxidation and metallothionein levels of developing rats exposed to cadmium and dexamethasone. Toxicology letters, 144(2), pp. 151-157.

MICHAELSEN, K. and LOHMANN, C., 2010. Calcium dynamics at developing synapses: mechanisms and functions. European Journal of Neuroscience, 32(2), pp. 218-223. 
MILATOVIC, D., YIN, Z., GUPTA, R.C., SIDORYK, M., ALBRECHT, J., ASCHNER, J.L. and ASCHNER, M., 2007. Manganese induces oxidative impairment in cultured rat astrocytes. Toxicological sciences, 98(1), pp. 198-205.

MILOS, M., SCHAER, J., COMTE, M. and COX, J.A., 1989. Evidence for four capital and six auxiliary cation-binding sites on calmodulin: divalent cation interactions monitored by direct binding and microcalorimetry. Journal of inorganic biochemistry, 36(1), pp. 1125.

MOEWS, P. and KRETSINGER, R., 1975. Refinement of the structure of carp muscle calcium-binding parvalbumin by model building and difference Fourier analysis. Journal of Molecular Biology, 91(2), pp. 201-225.

MORALES, K.A., LASAGNA, M., GRIBENKO, A.V., YOON, Y., REINHART, G.D., LEE, J.C., CHO, W., LI, P. and IGUMENOVA, T.I., 2011. Pb2 as modulator of proteinmembrane interactions. Journal of the American Chemical Society, 133(27), pp. 1059910611.

MORRIS, G.M., HUEY, R., LINDSTROM, W., SANNER, M.F., BELEW, R.K., GOODSELL, D.S. and OLSON, A.J., 2009. AutoDock4 and AutoDockTools4: Automated docking with selective receptor flexibility. Journal of computational chemistry, 30(16), pp. 2785-2791.

MOSS, S.E. and MORGAN, R.O., 2004. The annexins. Genome biology, 5(4), pp. 219.

MÜLLER, S., WALTER, R.D. and FAIRLAMB, A.H., 1995. Differential susceptibility of filarial and human erythrocyte glutathione reductase to inhibition by the trivalent organic arsenical melarsen oxide. Molecular and biochemical parasitology, 71(2), pp. 211-219.

MYSHKIN, A.E. and KHROMOVA, V.S., 2000. Histidin as a mercurial poisoning inhibitor. Biochemical and biophysical research communications, 273(3), pp. 816-819.

NALEFSKI, E.A. and FALKE, J.J., 1996. The C2 domain calcium-binding motif: structural and functional diversity. Protein Science, 5(12), pp. 2375-2390.

NEWTON, A.C., 1995. Protein kinase C: seeing two domains. Current Biology, 5(9), pp. 973-976.

NIWA, N. and NERBONNE, J.M., 2010. Molecular determinants of cardiac transient outward potassium current (Ito) expression and regulation. Journal of Molecular and Cellular Cardiology, 48(1), pp. 12-25.

NOBLE, W., PlANEL, E., ZEHR, C., OLM, V., MEYERSON, J., SULEMAN, F., GAYNOR, K., WANG, L., LAFRANCOIS, J., FEINSTEIN, B., BURNS, M., KRISHNAMURTHY, P., WEN, Y., BHAT, R., LEWIS, J., DICKSON, D. and DUFF, K., 
2005. Inhibition of glycogen synthase kinase- 3 by lithium correlates with reduced tauopathy and degeneration in vivo. Proceedings of the National Academy of Sciences of the United States of America, 102(19), pp. 6990-6995.

O'CALLAGHAN, D.W., IVINGS, L., WEISS, J.L., ASHBY, M.C., TEPIKIN, A.V. and BURGOYNE, R.D., 2002. Differential use of myristoyl groups on neuronal calcium sensor proteins as a determinant of spatio-temporal aspects of $\mathrm{Ca} 2+$ signal transduction. The Journal of biological chemistry, 277(16), pp. 14227-14237.

OKUDA, B., IWAMOTO, Y., TACHIBANA, H. and SUGITA, M., 1997. Parkinsonism after acute cadmium poisoning. Clinical neurology and neurosurgery, 99(4), pp. 263-265.

OLSHER, U., IZATT, R.M., BRADSHAW, J.S. and DALLEY, N.K., 1991. Coordination chemistry of lithium ion: a crystal and molecular structure review. Chemical reviews, 91(2), pp. 137-164.

ORTLUND, E., CHAI, G., GENGE, B. and WUTHIER, R., 2004. E., Lebioda, L.: Crystal structure of chicken annexin A5 in complex with functional modifiers $\mathrm{Ca} 2$ and $\mathrm{Zn} 2$ reveal Zn2 -induced formation of non-planar assemblies. Annexins, 1, pp. 183-190.

OSAWA, M., DACE, A., TONG, K.I., VALIVETI, A., IKURA, M. and AMES, J.B., 2005. $\mathrm{Mg} 2+$ and $\mathrm{Ca} 2+$ differentially regulate DNA binding and dimerization of DREAM. The Journal of biological chemistry, 280(18), pp. 18008-18014.

OSAWA, M., TONG, K.I., LILLIEHOOK, C., WASCO, W., BUXBAUM, J.D., CHENG, H.Y., PENNINGER, J.M., IKURA, M. and AMES, J.B., 2001. Calcium-regulated DNA binding and oligomerization of the neuronal calcium-sensing protein, calsenilin/DREAM/KChIP3. The Journal of biological chemistry, 276(44), pp. 4100541013.

ÖZERDEM, A., GÜNTEKIN, B., ATAGÜN, İ., TURP, B. and BAŞAR, E., 2011. Reduced long distance gamma $(28-48 \mathrm{~Hz})$ coherence in euthymic patients with bipolar disorder. Journal of affective disorders, 132(3), pp. 325-332.

PAGE, M.J. and DI CERA, E., 2006. Role of Na and K in enzyme function. Physiological Reviews, 86(4), pp. 1049-1092.

PANAYI, A., SPYROU, N., IVERSEN, B., WHITE, M. and PART, P., 2002. Determination of cadmium and zinc in Alzheimer's brain tissue using inductively coupled plasma mass spectrometry. Journal of the neurological sciences, 195(1), pp. 1-10.

PARK, J.D. and ZHENG, W., 2012. Human exposure and health effects of inorganic and elemental mercury. Journal of preventive medicine and public health = Yebang Uihakhoe chi, 45(6), pp. 344-352. 
PATEL, S.P., CAMPBELL, D.L. and STRAUSS, H.C., 2002. Elucidating KChIP effects on Kv4. 3 inactivation and recovery kinetics with a minimal KChIP2 isoform. The Journal of physiology, 545(1), pp. 5-11.

PESCH, B., HAERTING, J., RANFT, U., KLIMPEL, A., OELSCHLÄGEL, B. and SCHILL, W., 2000. Occupational risk factors for renal cell carcinoma: agent-specific results from a case-control study in Germany. International journal of epidemiology, 29(6), pp. 1014-1024.

PESHENKO, I.V. and DIZHOOR, A.M., 2006. Ca2+ and Mg2+ binding properties of GCAP-1. Evidence that Mg2+-bound form is the physiological activator of photoreceptor guanylyl cyclase. The Journal of biological chemistry, 281(33), pp. 23830-23841.

PESHENKO, I.V. and DIZHOOR, A.M., 2004. Guanylyl cyclase-activating proteins (GCAPs) are $\mathrm{Ca} 2+\mathrm{Mg} 2+$ sensors: implications for photoreceptor guanylyl cyclase (RetGC) regulation in mammalian photoreceptors. The Journal of biological chemistry, 279(17), pp. 16903-16906.

PHAM, K., DHULIPALA, G., GONZALEZ, W.G., GERSTMAN, B.S., REGMI, C., CHAPAGAIN, P.P. and MIKSOVSKA, J., 2015. Ca2 and Mg2 modulate conformational dynamics and stability of downstream regulatory element antagonist modulator. Protein Science, 24(5), pp. 741-751.

PHAM, K. and MIKSOVSKA, J., 2016. Molecular insight of DREAM and presenilin 1 Cterminal fragment interactions. FEBS letters, 590(8), pp. 1114-1122.

PHILLIPS, J.C., BRAUN, R., WANG, W., GUMBART, J., TAJKHORSHID, E., VILLA, E., CHIPOT, C., SKEEL, R.D., KALE, L. and SCHULTEN, K., 2005. Scalable molecular dynamics with NAMD. Journal of computational chemistry, 26(16), pp. 1781-1802.

PIHL, R.O. and PARKES, M., 1977. Hair element content in learning disabled children. Science (New York, N.Y.), 198(4313), pp. 204-206.

PIOLETTI, M., FINDEISEN, F., HURA, G.L. and MINOR, D.L., 2006. Threedimensional structure of the KChIP1-Kv4. $3 \mathrm{~T} 1$ complex reveals a cross-shaped octamer. Nature structural \& molecular biology, 13(11), pp. 987-995.

PONGS, O., LINDEMEIER, J., ZHU, X., THEIL, T., ENGELKAMP, D., KRAHJENTGENS, I., KOCH, K., SCHWEMER, J., RIVOSECCHI, R. and MALLART, A., 1993. Frequenin - a novel calcium-binding protein that modulates synaptic efficacy in the Drosophila nervous system. Neuron, 11(1), pp. 15-28.

RAMACHANDRAN, P.L., CRAIG, T.A., ATANASOVA, E.A., CUI, G., OWEN, B.A., BERGEN, H.R.,3RD, MER, G. and KUMAR, R., 2012. The potassium channel interacting 
protein 3 (DREAM/KChIP3) heterodimerizes with and regulates calmodulin function. The Journal of biological chemistry, 287(47), pp. 39439-39448.

RAO, S., SATYSHUR, K., GREASER, M. and SUNDARALINGAM, M., 1996. X-ray structures of $\mathrm{Mn}, \mathrm{Cd}$ and $\mathrm{Tb}$ metal complexes of troponin C. Acta Crystallographica Section D: Biological Crystallography, 52(5), pp. 916-922.

RATNAIKE, R.N., 2003. Acute and chronic arsenic toxicity. Postgraduate medical journal, 79(933), pp. 391-396.

REDMOND, L. and GHOSH, A., 2005. Regulation of dendritic development by calcium signaling. Cell calcium, 37(5), pp. 411-416.

RESCHER, U. and GERKE, V., 2004. Annexins--unique membrane binding proteins with diverse functions. Journal of cell science, 117(Pt 13), pp. 2631-2639.

RIGON, A.P., CORDOVA, F.M., OLIVEIRA, C.S., POSSER, T., COSTA, A.P., SILVA, I.G., SANTOS, D.A., ROSSI, F.M., ROCHA, J.B.T. and LEAL, R.B., 2008. Neurotoxicity of cadmium on immature hippocampus and a neuroprotective role for p38MAPK. Neurotoxicology, 29(4), pp. 727-734.

ROBISON, G., ZAKHAROVA, T., FU, S., JIANG, W., FULPER, R., BARREA, R., MARCUS, M.A., ZHENG, W. and PUSHKAR, Y., 2012. X-ray fluorescence imaging: a new tool for studying manganese neurotoxicity. PloS one, 7(11), pp. e48899.

ROCKENSTEIN, E., TORRANCE, M., ADAME, A., MANTE, M., BAR-ON, P., ROSE, J.B., CREWS, L. and MASLIAH, E., 2007. Neuroprotective effects of regulators of the glycogen synthase kinase-3beta signaling pathway in a transgenic model of Alzheimer's disease are associated with reduced amyloid precursor protein phosphorylation. The Journal of neuroscience : the official journal of the Society for Neuroscience, 27(8), pp. 1981-1991.

ROELS, H., BERNARD, A.M., CARDENAS, A., BUCHET, J.P., LAUWERYS, R.R., HOTTER, G., RAMIS, I., MUTTI, A., FRANCHINI, I. and BUNDSCHUH, I., 1993. Markers of early renal changes induced by industrial pollutants. III. Application to workers exposed to cadmium. British journal of industrial medicine, 50(1), pp. 37-48.

ROWLAND, L.P. and SHNEIDER, N.A., 2001. Amyotrophic lateral sclerosis. New England Journal of Medicine, 344(22), pp. 1688-1700.

RUDOLPH, W.W. and PYE, C.C., 1998. Raman spectroscopic measurements and ab initio molecular orbital studies of cadmium (II) hydration in aqueous solution. The Journal of Physical Chemistry B, 102(18), pp. 3564-3573. 
SAIJOH, K., INOUE, Y., KATSUYAMA, H. and SUMINO, K., 1988. The interaction of cations with activity of soluble protein kinase $\mathrm{C}$ from mouse brain. Pharmacology \& toxicology, 63(4), pp. 221-224.

SATARUG, S. and MOORE, M.R., 2004. Adverse health effects of chronic exposure to low-level cadmium in foodstuffs and cigarette smoke. Environmental health perspectives, 112(10), pp. 1099-1103.

SCANNEVIN, R.H., WANG, K., JOW, F., MEGULES, J., KOPSCO, D.C., EDRIS, W., CARROLL, K.C., LÜ, Q., XU, W. and XU, Z., 2004. Two N-terminal domains of Kv4 K channels regulate binding to and modulation by KChIP1. Neuron, 41(4), pp. 587-598.

SCHLECKER, C., BOEHMERLE, W., JEROMIN, A., DEGRAY, B., VARSHNEY, A., SHARMA, Y., SZIGETI-BUCK, K. and EHRLICH, B.E., 2006a. Neuronal calcium sensor-1 enhancement of InsP3 receptor activity is inhibited by therapeutic levels of lithium. The Journal of clinical investigation, 116(6), pp. 1668-1674.

SCHLECKER, C., BOEHMERLE, W., JEROMIN, A., DEGRAY, B., VARSHNEY, A., SHARMA, Y., SZIGETI-BUCK, K. and EHRLICH, B.E., 2006b. Neuronal calcium sensor-1 enhancement of InsP3 receptor activity is inhibited by therapeutic levels of lithium. The Journal of clinical investigation, 116(6), pp. 1668-1674.

SCHNEIDER, J., ANDERSON, D., SONNENAHALLI, H. and VADIGEPALLI, R., 2011. Sex-based differences in gene expression in hippocampus following postnatal lead exposure. Toxicology and applied pharmacology, 256(2), pp. 179-190.

SCOTT, D.L., WHITE, S.P., OTWINOWSKI, Z., YUAN, W., GELB, M.H. and SIGLER, P.B., 1990. Interfacial catalysis: the mechanism of phospholipase A2. Science (New York, N.Y.), 250(4987), pp. 1541-1546.

SENATOROV, V., REN, M., KANAI, H., WEI, H. and CHUANG, D., 2004. Short-term lithium treatment promotes neuronal survival and proliferation in rat striatum infused with quinolinic acid, an excitotoxic model of Huntington's disease. Molecular psychiatry, 9(4), pp. 371.

SENGUEN, F.T. and GRABAREK, Z., 2012. X-ray structures of magnesium and manganese complexes with the N-terminal domain of calmodulin: insights into the mechanism and specificity of metal ion binding to an EF-hand. Biochemistry, 51(31), pp. 6182-6194.

STEVENS, F.C., WALSH, M., HO, H.C., TEO, T.S. and WANG, J.H., 1976. Comparison of calcium-binding proteins. Bovine heart and brain protein activators of cyclic nucleotide phosphodiesterase and rabbit skeletal muscle troponin C. The Journal of biological chemistry, 251(15), pp. 4495-4500. 
STRATTON, A., ERICKSEN, M., HARRIS, T.V., SYMMONDS, N. and SILVERSTEIN, T.P., 2017. Mercury (II) binds to both of chymotrypsin's histidines, causing inhibition followed by irreversible denaturation/aggregation. Protein Science, 26(2), pp. 292-305.

SU, T., CONG, W., LONG, Y., LUO, A., SUN, W., DENG, W. and LIAO, W., 2008. Altered expression of voltage-gated potassium channel 4.2 and voltage-gated potassium channel 4-interacting protein, and changes in intracellular calcium levels following lithium-pilocarpine-induced status epilepticus. Neuroscience, 157(3), pp. 566-576.

SU, Y., RYDER, J., LI, B., WU, X., FOX, N., SOLENBERG, P., BRUNE, K., PAUL, S., ZHOU, Y. and LIU, F., 2004. Lithium, a common drug for bipolar disorder treatment, regulates amyloid- $\beta$ precursor protein processing. Biochemistry, 43(22), pp. 6899-6908.

SÜDHOF, T.C., 2004. The synaptic vesicle cycle. Annu.Rev.Neurosci., 27, pp. 509-547.

SUMMERS, A.O., 2009. Damage control: regulating defenses against toxic metals and metalloids. Current opinion in microbiology, 12(2), pp. 138-144.

SUZUKI, Y., CHAO, S., ZYSK, J.R. and CHEUNG, W.Y., 1985. Stimulation of calmodulin by cadmium ion. Archives of Toxicology, 57(3), pp. 205-211.

TANAKA, D., NAKADA, K., TAKAO, K., OGASAWARA, E., KASAHARA, A., SATO, A., YONEKAWA, H., MIYAKAWA, T. and HAYASHI, J., 2008. Normal mitochondrial respiratory function is essential for spatial remote memory in mice. Molecular Brain, 1(1), pp. 21.

TANAKA, T., AMEST, J.B., HARVEY, T.S. and STRYER, L., 1995. Sequestration of the membrane-targeting myristoyl group of recoverin in the calcium-free state. Nature, 376(6539), pp. 444.

TELEMAN, O., DRAKENBERG, T., FORSÉN, S. and THULIN, E., 1983. Calcium and Cadmium Binding to Troponin C: Evidence for Cooperativity. European journal of biochemistry, 134(3), pp. 453-457.

THATCHER, R., LESTER, M., MCALASTER, R. and HORST, R., 1982. Effects of low levels of cadmium and lead on cognitive functioning in children. Archives of Environmental Health: An International Journal, 37(3), pp. 159-166.

THATCHER, R., MCALASTER, R. and LESTER, M., 1984. Evoked Potentials Related to Hair Cadmium and Lead in Children a. Annals of the New York Academy of Sciences, 425(1), pp. 384-390.

THEVENOD, F. and JONES, S.W., 1992. Cadmium block of calcium current in frog sympathetic neurons. Biophysical journal, 63(1), pp. 162-168. 
TONG, Y., YANG, H., TIAN, X., WANG, H., ZHOU, T., ZHANG, S., YU, J., ZHANG, T., FAN, D. and GUO, X., 2014. High manganese, a risk for Alzheimer's disease: high manganese induces amyloid- $\beta$ related cognitive impairment. Journal of Alzheimer's Disease, 42(3), pp. 865-878.

TUSCHL, K., MILLS, P.B. and CLAYTON, P.T., 2013. Manganese and the brain. International review of neurobiology. Elsevier, pp. 277-312.

VANSCYOC, W.S., SORENSEN, B.R., RUSINOVA, E., LAWS, W.R., ROSS, J.A. and SHEA, M.A., 2002. Calcium binding to calmodulin mutants monitored by domain-specific intrinsic phenylalanine and tyrosine fluorescence. Biophysical journal, 83(5), pp. 27672780 .

VIG, P., NATH, R. and DESAIAH, D., 1989. Metal inhibition of calmodulin activity in monkey brain. Journal of Applied Toxicology, 9(5), pp. 313-316.

VOGEL, H.J., 1994. Calmodulin: a versatile calcium mediator protein. Biochemistry and cell biology, 72(9-10), pp. 357-376.

VON BRÖMSSEN, M., JAKARIYA, M., BHATTACHARYA, P., AHMED, K.M., HASAN, M.A., SRACEK, O., JONSSON, L., LUNDELL, L. and JACKS, G., 2007. Targeting low-arsenic aquifers in Matlab Upazila, southeastern Bangladesh. Science of the Total Environment, 379(2-3), pp. 121-132.

VON LEWINSKI, F. and KELLER, B.U., 2005. Ca2 , mitochondria and selective motoneuron vulnerability: implications for ALS. Trends in neurosciences, 28(9), pp. 494500 .

VOSAHLIKOVA, M. and SVOBODA, P., 2016. Lithium-therapeutic tool endowed with multiple beneficiary effects caused by multiple mechanisms. Acta Neurobiol Exp, 76, pp. $1-19$.

WAALKES, M.P., 2000. Cadmium carcinogenesis in review. Journal of inorganic biochemistry, 79(1-4), pp. 241-244.

WADA, A., YOKOO, H., YANAGITA, T. and KOBAYASHI, H., 2005. Lithium: potential therapeutics against acute brain injuries and chronic neurodegenerative diseases. Journal of pharmacological sciences, 99(4), pp. 307-321.

WALTER, I., SCHWERDTLE, T., THUY, C., PARSONS, J.L., DIANOV, G.L. and HARTWIG, A., 2007. Impact of arsenite and its methylated metabolites on PARP-1 activity, PARP-1 gene expression and poly (ADP-ribosyl) ation in cultured human cells. DNA repair, 6(1), pp. 61-70. 
WANG, B. and DU, Y., 2013. Cadmium and its neurotoxic effects. Oxidative medicine and cellular longevity, 2013.

WANG, H., ZHANG, L., ABEL, G.M., STORM, D.R. and XIA, Z., 2017. Cadmium exposure impairs cognition and olfactory memory in male C57BL/6 mice. Toxicological Sciences, 161(1), pp. 87-102.

WANG, H., YAN, Y., LIU, Q., HUANG, Y., SHEN, Y., CHEN, L., CHEN, Y., YANG, Q., HAO, Q. and WANG, K., 2007a. Structural basis for modulation of Kv4 K channels by auxiliary KChIP subunits. Nature neuroscience, 10(1), pp. 32.

WANG, H., YAN, Y., LIU, Q., HUANG, Y., SHEN, Y., CHEN, L., CHEN, Y., YANG, Q., HAO, Q. and WANG, K., 2007b. Structural basis for modulation of Kv4 K channels by auxiliary KChIP subunits. Nature neuroscience, 10(1), pp. 32.

WAXMAN, S. and ANDERSON, K.C., 2001. History of the development of arsenic derivatives in cancer therapy. The oncologist, 6 Suppl 2, pp. 3-10.

WEBB, J.L., 1966. Enzyme and Metabolic Inhibitors: Malonate, Analogs, Dehydroacetate. Academic Press.

WEI, H., QIN, Z., SENATOROV, V., WEI, W., WANG, Y., QIAN, Y. and CHUANG, D., 2001. Lithium suppresses excitotoxicity-induced striatal lesions in a rat model of Huntington's disease. Neuroscience, 106(3), pp. 603-612.

WEISSKOPF, M.G., HU, H., MULKERN, R.V., WHITE, R., ARO, A., OLIVEIRA, S. and WRIGHT, R.O., 2004. Cognitive deficits and magnetic resonance spectroscopy in adult monozygotic twins with lead poisoning. Environmental health perspectives, 112(5), pp. 620-625.

WENG, X., LUECKE, H., SONG, I.S., KANG, D.S., KIM, S. and HUBER, R., 1993. Crystal structure of human annexin I at $2.5 \AA$ resolution. Protein Science, 2(3), pp. 448458.

WILLIAMS, R.S. and HARWOOD, A.J., 2000. Lithium therapy and signal transduction. Trends in pharmacological sciences, 21(2), pp. 61-64.

WONG, K. and KLAASSEN, C.D., 1982. Neurotoxic effects of cadmium in young rats. Toxicology and applied pharmacology, 63(3), pp. 330-337.

WU, J., BASHA, M.R., BROCK, B., COX, D.P., CARDOZO-PELAEZ, F., MCPHERSON, C.A., HARRY, J., RICE, D.C., MALONEY, B., CHEN, D., LAHIRI, D.K. and ZAWIA, N.H., 2008. Alzheimer's disease (AD)-like pathology in aged monkeys after infantile exposure to environmental metal lead $(\mathrm{Pb})$ : evidence for a developmental 
origin and environmental link for AD. The Journal of neuroscience : the official journal of the Society for Neuroscience, 28(1), pp. 3-9.

YOSHIDA, S., 2001. Re-evaluation of acute neurotoxic effects of $\mathrm{Cd} 2$ on mesencephalic trigeminal neurons of the adult rat. Brain research, 892(1), pp. 102-110.

YOUDIM, M.B. and ARRAF, Z., 2004. Prevention of MPTP (N-methyl-4-phenyl-1, 2, 3, 6-tetrahydropyridine) dopaminergic neurotoxicity in mice by chronic lithium: involvements of Bcl-2 and Bax. Neuropharmacology, 46(8), pp. 1130-1140.

YU, Q. and ZHOU, Y.Z., 2018. High level of Mn in brain is a risk for Alzheimer disease. Sheng li xue bao : [Acta physiologica Sinica], 70(2), pp. 193-200.

ZHANG, X.W., YAN, X.J., ZHOU, Z.R., YANG, F.F., WU, Z.Y., SUN, H.B., LIANG, W.X., SONG, A.X., LALLEMAND-BREITENBACH, V., JEANNE, M., ZHANG, Q.Y., YANG, H.Y., HUANG, Q.H., ZHOU, G.B., TONG, J.H., ZHANG, Y., WU, J.H., HU, H.Y., DE THE, H., CHEN, S.J. and CHEN, Z., 2010. Arsenic trioxide controls the fate of the PML-RARalpha oncoprotein by directly binding PML. Science (New York, N.Y.), 328(5975), pp. 240-243.

ZHAO, L., CHEN, S., JIA, L., SHU, S., ZHU, P. and LIU, Y., 2012. Selectivity of arsenite interaction with zinc finger proteins. Metallomics, 4(9), pp. 988-994.

ZOZULYA, S. and STRYER, L., 1992. Calcium-myristoyl protein switch. Proceedings of the National Academy of Sciences of the United States of America, 89(23), pp. 1156911573. 
VITA

SAMIOL AZAM

EDUCATION AND AWARDS

Aug 2014 - Nov 2019

Doctor of Philosophy in Chemistry

Florida International University

Miami, Florida USA.

2004-2009

Bachelor of Science in Chemistry

University of Dhaka

Dhaka, Bangladesh.

Awarded highly competitive dissertation year fellowship (DYF) from Florida International University (FIU).

Travel Award from South Florida Section of American Chemical Society for attending ACS National Meeting 2019 in Orlando, FL.

Travel Award from Florida International University (FIU) Graduate and Professional Student Committee (GPSC) and College of Arts, Sciences, and Education (CASE) for attending Biophysical Society Annual Meeting 2019 in Baltimore, MD.

Travel Award from FIU GPSC and CASE for attending Biophysical Society Annual Meeting 2018 in San Francisco, CA.

Travel Award from FIU GPSC for attending Biophysical Society Annual Meeting 2017 in New Orleans, LA.

\section{SELECTED PUBLICATIONS AND PRESENTATIONS}

Azam, Samiol; Gessica St Louis; Miksovska, Jaroslava; (2019) Cadmium association to DREAM promotes DREAM interactions with intracellular partners in a similar manner as its physiological ligand, calcium. Metallomics; DOI: 10.1039/C9MT00059C. Impact factor 4.06.

Azam, Samiol; Miksovska, Jaroslava; (2019) $\mathrm{Pb}^{2+}$ binds to DREAM and modulates its interactions with binding partners: A link between neuronal calcium sensors and $\mathrm{Pb}^{2+}$ neurotoxicity. ACS Chemical Neuroscience; 10 (3), 1263-1272. Impact factor: 4.21.

Azam, Samiol; Miksovska, Jaroslava; Effect of $\mathrm{Li}^{+}$Binding on Secondary and Tertiary Structures, Hydrophobicity, Thermodynamics, and Interactions with Interacting Partners of DREAM. ACS National Meeting in Orlando, FL, April 2019 (oral). 
Azam, Samiol; Miksovska, Jaroslava; The Effect of $\mathrm{Li}^{+}$Binding on Secondary and Tertiary Structures, Hydrophobicity, Thermodynamics, and Interactions with Interacting Partners of DREAM. Annual Meeting of The Biophysical Society in Baltimore, MD, March 2019 (poster).

Azam, Samiol; Miksovska, Jaroslava; Cadmium Associates to DREAM and Alters Its Interactions with Intracellular Partners. ACS National Meeting in Boston, MA, August 2018 (poster).

Azam, Samiol; Miksovska, Jaroslava; The Effect of lithium Binding on Secondary and Tertiary Structures, Hydrophobicity, Thermodynamics, and Interactions with Interacting Partners of DREAM. Florida Annual Meeting and Exposition (FAME), organized by Florida Local Section of the American Chemical Society, Tampa, FL, May 2018 (oral).

Azam, Samiol; Miksovska, Jaroslava; Cadmium Associates to DREAM and Alters Its Interactions with Intracellular Partners. FAME, Tampa, FL, May 2018 (poster).

Azam, Samiol; Miksovska, Jaroslava; Role of Lys Residue at Position 87 of DREAM in Allosteric Regulation of DREAM Interactions with Kv Channel. Annual Meeting of The Biophysical Society in San Francisco, CA, February 2018 (poster).

Azam, Samiol; Miksovska, Jaroslava; Lead Tightly Associates to Neuronal Calcium Sensor (NCS) Protein DREAM and Promotes Structural Changes Analogous to CalciumBound DREAM. ACS National Meeting in Washington, DC, August 2017 (poster).

Azam, Samiol; Miksovska, Jaroslava; Lead Tightly Associates to Neuronal Calcium Sensor (NCS) Protein DREAM and Promotes Structural Changes Analogous to CalciumBound DREAM. FAME, Tampa, FL, May 2017 (poster).

Azam, Samiol; Miksovska, Jaroslava; Lead Tightly Associates to Neuronal Calcium Sensor (NCS) Protein DREAM and Promotes Structural Changes Analogous to CalciumBound DREAM. Annual Meeting of The Biophysical Society in New Orleans, LA, February 2017 (poster). 